\title{
Measurements of Turbulence at High Reynolds Numbers
}

\author{
From Eulerian Statistics Towards Lagrangian Particle \\ TRACKING
}

\author{
Dissertation \\ for the award of the degree \\ "Doctor rerum naturalium" \\ of the Georg-August-Universität Göttingen
}

within the doctoral degree programme

Physics of Biological and Complex Systems

of the Göttingen Graduate School of Neurosciences, Biophysics, and

Molecular Biosciences (GGNB)

of the Georg-August University School of Sciences (GAUSS)

\author{
submitted by \\ Christian Küchler \\ from Bruchköbel
}

Göttingen, October 2020 


\section{Thesis Committee}

Prof. Dr. Dr. h.c. Eberhard Bodenschatz

Max-Planck-Institut für Dynamik und Selbstorganisation

Georg-August-Universität Göttingen

Dr. Michael Wilczek

Max-Planck-Institute for Dynamics and Self-Organization

Prof. Dr. Jens Niemeyer

Georg-August-Universität Göttingen

\section{Members of the Examination Board}

Referee: Prof. Dr. Dr. h.c. Eberhard Bodenschatz

Max-Planck-Institut für Dynamik und Selbstorganisation

Georg-August-Universität Göttingen

$2^{\text {nd }}$ Referee: Dr. Michael Wilczek

Max-Planck-Institut für Dynamik und Selbstorganisation

\section{Further Members of the Examination Board}

Prof. Dr. Jens Niemeyer

Georg-August-Universität Göttingen

Prof. Dr. Dr.-Ing. habil. Andreas Dillmann

Deutsches Zentrum für Luft- und Raumfahrt

Georg-August-Universität Göttingen

Prof. Dr. Andreas Tilgner

Georg-August-Universität Göttingen

Dr. habil. Olga Shishkina

Max-Planck-Institut für Dynamik und Selbstorganisation

Date of the oral examination: 16.12 .2020 
Revised version, March 2021 


\section{Abstract}

The effective description and fundamental understanding of turbulent flows remains elusive to modern physics despite centuries of research and its great importance in numerous fields. The theoretical difficulties of the topic (nonlinear, nonlocal, or unclosed equations) are accompanied by the multiscale characteristics, large number of degrees of freedom, and strong sensitivity to initial conditions that make numerical and laboratory experiments equally challenging. One potential way to unravel the dynamics underlying turbulent motions is the separation of inertial forces from viscous forces, i.e. the study of turbulence at very large Reynolds numbers. The Max Planck Variable Density Turbulence Tunnel (VDTT) is a facility well-suited for the study of such large Reynolds numbers under controlled conditions. Its active grid allows the creation of turbulence at Taylor-scale Reynolds numbers $R_{\lambda}>6000$ that can be investigated with state-of-the-art subminiature hot wires and whose turbulence generation can be controlled with great flexibility. This allows the study of fine details of the turbulence energy spectrum, such as the bottleneck effect, which are difficult to investigate even at small $R_{\lambda}$. We show for the first time experimentally that the bottleneck effect decreases with increasing Reynolds number up to $R_{\lambda} \approx 5000$ confirming previous numerical studies at lower Reynolds numbers.

A very influential phenomenological model is the seminal self-similar model of the velocity increment statistics by Kolmogorov ${ }^{1}$ and its intermittency refinements ${ }^{2}$. In this thesis the cornerstones of this scaling theory are confirmed approximately throughout the range of $R_{\lambda}$ studied (150-6000) using hot wire data from the VDTT. This constitutes the most extensive dataset in this range of $R_{\lambda}$ to the author's best knowledge. The local scaling exponents of the increment statistics becomes $R_{\lambda}$-independent for $R_{\lambda}>2000$. They do however not allow the immediate identification of an inertial range scaling exponent,

\footnotetext{
${ }^{1}$ A. Kolmogorov. "The Local Structure of Turbulence in Incompressible Viscous Fluid for Very Large Reynolds' Numbers". In: Dokl. Akad. Nauk SSSR 30 (1941), pp. 301-305.

${ }^{2}$ U. Frisch. Turbulence. Cambridge University Press, Nov. 1995. DoI: 10 . 1017 / CB09781139170666.
} 
but carry the imprints of the turbulence decay and certain dissipative effects. The effect of decay is more dramatic, but can be explained by a model ${ }^{3}$ for the statistics of decaying turbulence. This allows the extraction of an inertial range scaling exponent that agrees with those obtained by the extended self-similarity technique ${ }^{4}$. The dissipative effects take the form of log-periodic oscillations on the scaling functions, whose exact physical origin remains elusive.

The remainder of the thesis deals with the design and implementation of a particle tracking system in the VDTT. The system allows the measurement of statistics in the Lagrangian framework, where instead of a multi-location measurement, individual fluid parcels are followed throughout the flow and multi-time statistics are obtained. The setup records the motion of cellulose particles of Stokes numbers between 0.0001 and 2 illuminated by a high-power laser using four stationary high-speed cameras. It is shown that the setup is capable of acquiring acceleration statistics and record particle tracks of up to 15 viscous time scales. This allows the systematic investigation of Lagrangian turbulence at $R_{\lambda}>2000$ where such investigations were impossible heretofore.

3P.-F. Yang, A. Pumir, and H. Xu. "Generalized self-similar spectrum and the effect of large-scale in decaying homogeneous isotropic turbulence". In: New J. Phys. 20.10 (Oct. 2018), p. 103035. DOI: 10.1088/1367-2630/aae72d.

4R. Benzi et al. "Extended self-similarity in turbulent flows". In: Phys. Rev. E 48.1 (1993), pp. 29-32. DOI: 10.1103/PhysRevE. 48.R29. 


\section{Contents}

$\begin{array}{lll}\text { Abstract } & \text { iv }\end{array}$

Contents $\mathbf{v}$

I Introduction 1

1 Definition and Motivation 3

1.1 Defining Turbulence ................. 3

1.2 Motivation for Studying Turbulence (at High Reynolds Numbers) 4

1.3 Modern Turbulence Research at High $R_{\lambda} \ldots \ldots \ldots$

1.4 This Thesis .................... 8

2 The Equations of Motion 9

2.1 The Navier-Stokes Equations ............... . . 9

2.2 Nonlocality of the Pressure Term . . . . . . . . . . . . . 11

2.3 The Energy Equation and Dissipation . . . . . . . . . . II

2.4 Vorticity ............................ 13

3 The Statistics of Homogeneous Isotropic Turbulence 15

3.1 Three Statistical Objects . . . . . . . . . . . . . . . . 15

3.2 The Karman-Howarth-Equation . . . . . . . . . . . . 17

3.3 Phenomenology and Scaling .............. 19

3.4 Lagrangian Turbulence . . . . . . . . . . . . . . . 27

4 Experimental Setup and Flow Properties 31

4.1 The Variable Density Turbulence Tunnel . . . . . . . . . . . . . 31

4.2 The Active Grid . . . . . . . . . . . . . . . . . . . . . . . . . . . . . .

4.3 Velocity Distributions . . . . . . . . . . . . . . . . 39

4.4 Decay of Turbulent Kinetic Energy . . . . . . . . . . . . . 43

4.5 Flow Profiles . . . . . . . . . . . . . . . . . 44 
4.6 Summary . . . . . . . . . . . . . . . . . . . 49

Bibliography

II Eulerian Measurements 63

5 On Hot Wire Measurements 65

5.1 Introduction to Hot Wire Anemometry . . . . . . . . . . 65

5.2 Taylor's Hypothesis . . . . . . . . . . . . . . . . . . . 73

5.3 Measurement Protocol and Data Analysis . . . . . . . . . 79

6 Scaling In Decaying High-Reynolds Number Turbulence 85

7 Extension of Eulerian Predictions to High $R_{\lambda} \quad 97$

7.1 The Dissipation Constant . . . . . . . . . . . . . . . . 97

7.2 The Kolmogorov Constant and the 4/5-th Law . . . . . . . . 99

$7 \cdot 3$ ESS-Exponents at large $R_{\lambda} \ldots \ldots \ldots \ldots \ldots \ldots \ldots$

8 Log-Periodic Oscillations in Even-Order Structure Functions $\quad 107$ 8.1 Introduction . . . . . . . . . . . . . . . . . 107

8.2 Log-Periodic Oscillations in Even-Order Structure Functions . . 109

8.3 Discussion . . . . . . . . . . . . . . . 112

9 Experimental Study of the Bottleneck Effect in Fully Developed Turbulence

Bibliography

\section{Towards Lagrangian Particle Tracking}

10 Lagrangian Particle Tracking in the Variable Density Turbulence Tunnel 149

10.1 Design Considerations . . . . . . . . . . . . . . . . . . . 149

10.2 Realisation of a Lagrangian Particle Tracking Setup . . . . . 155

11 Results of Particle Tracking Experiments in the VDTT 167

11.1 Characterisation of the Data and Data Analysis . . . . . . . 167

11.2 Velocity Statistics . . . . . . . . . . . . . . . . . 172

11.3 Acceleration Statistics of Lagrangian Tracks . . . . . . . . . . . . . . . . . . . . . . . . . . .

11.4 Discussion . . . . . . . . . . . . . . . . . . . . 175 
$\begin{array}{ll}\text { IV Discussion and Outlook } & 181\end{array}$

$\begin{array}{ll}12 \text { Discussion } & 183\end{array}$

12.1 Large Reynolds Numbers in the VDTT . . . . . . . . . . . . 183

12.2 High-Resolution Measurements of Turbulence . . . . . . . . . 186

12.3 Inertial Range Universality . . . . . . . . . . . . . . . . . 189

12.4 Towards Statistics of the Small Scales . . . . . . . . . . . . . . 192

$\begin{array}{lr}13 \text { Outlook } & 195\end{array}$

$\begin{array}{lr}\text { Bibliography } & 199\end{array}$

$\begin{array}{ll}\text { Acknowledgements } & 205\end{array}$

$\begin{array}{ll}\text { Appendices } & 207\end{array}$

Appendix A: Parts of the particle tracking system 209

$\begin{array}{ll}\text { Appendix B: Hot Wire Datasets } & 213\end{array}$

Appendix C: Fabrication of free-standing Pt nanowires for use as thermal anemometry probes in turbulence measurements 219

$\begin{array}{ll}\text { Curriculum Vitae } & 243\end{array}$ 


\section{Part I}

\section{Introduction}





\section{Chapter 1}

\section{Definition and Motivation}

\subsection{Defining Turbulence}

"Turbulence" is a rare case of an object of active physical research in the 21st century that immediately creates a picture or an idea in the mind of most people. This picture might be the whirls of a small wild river, an unpleasant airplane ride, or simply water flowing out of a fully opened tap. It is part of the fascination of the topic that despite a common intuition about turbulence and its importance to be described in the next section, scientific approaches to the subject notoriously face extreme challenges both theoretically and technologically yet to be overcome. A sufficiently general and effective theory of turbulence is yet to be found [1] and might elude us for a long time.

It is thus perhaps not surprising that even a formal definition beyond "I know it when I see it" is difficult to find. A collection of approaches can be found in the appendix of Ref. [2]. Here, the following definition is offered based on the famous experiment by Osbourne Reynolds [3] in 1880. He studied the flow of water through a glass pipe of diameter $D$. The flow rate (and thus the mean flow speed $U$ ) was controlled by an inlet valve. The viscosity of the water $v$ could be controlled by changing its temperature. Reynolds observed that two distinct flow states develop depending on the choice of these parameters by adding a line of dye into the fluid. At low flow speeds, in small pipes and at high viscosity, the dye flowed parallel to the glass pipe. The flow was well-predictable and not very complex. We call this a laminar flow. Under certain circumstances described by the pipe's Reynolds number

$$
\operatorname{Re}=\frac{U D}{v}
$$

the flow became "sinuous" (the term "turbulence" was introduced by Lord 
Kelvin only four years later) and the dye quickly mixed across the pipe diameter. This is the turbulent flow state. By carefully incrementing the experimental parameters, he found turbulence to prevail if $\operatorname{Re}>2000$. This number defining the onset of turbulence was recently refined to be $2040 \pm 10$ [4] indicating a very high quality of the original experiment almost 150 years ago.

A more general description should be concerned with the properties of turbulent flows, in particular its large number of degrees of freedom, the intermittent distribution of vorticity, its strong dependence on initial conditions, its dissipative nature and the large number of time- and length scales present.

\subsection{Motivation for Studying Turbulence (at High Reynolds Numbers)}

The prime motivations for the study of turbulence are - apart from scientific curiosity - the almost countless realisations of the phenomenon in nature. The earth is enclosed by the atmosphere - a huge turbulent multiphase flow. Its large-scale flow structures shape our climate and weather. The turbulent mixing inside a cloud is the prime suspect for the initiation of droplet growth that ultimately leads to rainfall [5]. Similarly, the organisation of the turbulent oceanic flows [6] has a great, though often underappreciated impact on our climate through coupling to the atmosphere [7], transport warm waters over thousands of kilometers or by influencing the growth of oxygen-producing ocean organisms on the small scale $[8,9]$. Realising the inherent complexity of turbulence and the lack of an efficient theory of the phenomenon, the uncertainties of climate and weather forecasts are easy to explain.

In engineering, turbulent flows are equally omnipresent. The scale-thinning properties of turbulence allow the design of efficient airfoils that carry the globalised economy. At the same time, turbulent drag is responsible for large amounts of the associated carbon emissions [10] and noise pollution. Yet, the properties of turbulence as an extremely efficient mixer allow for more efficient combustion engines that release less noise and emissions.

Turbulent flows also occur in the context of the human body: The carbonrich air we exhale is mixed sufficiently with fresh, oxygen rich air within about a second. The turbulent mixing is so efficient that we normally generate the necessary flow subconsciously without much effort. In contrast, turbulence in blood vessels can cause a deadly condition and some mechanisms that trigger 
turbulent patches have been discovered only recently [11].

This incomplete list is concluded with stating that carbon-free ways of energy harvesting such as wind power $[12,13,14,15]$ or nuclear fusion [16, 17] would benefit considerably from a better knowledge of turbulence.

Most examples offered here belong to a class of flows, where turbulent kinetic energy exists on many different time- and length scales. That is to say that the scale of the energy source is much larger than the scale of viscous dissipation. This means that an intermediate range of scales is likely to exist, where neither viscous dissipation, nor the flow boundary conditions play a major role. It is intuitive that the separation of these effects is desirable for a theoretic understanding of the governing dynamics.

\subsection{Modern Turbulence Research at High $R_{\lambda}$}

This section will give a brief overview of the state-of-the-art experiments known to the author that explicitly aim at creating turbulence at high Reynolds numbers. It is not a historical review, but focused on current efforts. To compare the different experimental setups, it is most useful to introduce a Reynolds number that does not depend on the specific flow geometry. This is the Taylor-scale Reynolds number $R_{\lambda}$ defined through the RMS of the velocity fluctuations $u_{R M S}$ (the residual of a velocity signal after subtracting its mean), the kinematic viscosity and the Taylor length scale $\lambda$ [18]. $\lambda$ can be seen as the typical length scale for an eddy dominated by inertial energy transfer. It is closely related to the average length between two zero-crossings of the velocity fluctuation signal [19]. The transition Reynolds number $\operatorname{Re}=2020$ of the pipe flow mentioned in Sec. I.I would correspond to $R_{\lambda} \sim 100$. To put into context, $R_{\lambda}$ of an atmospheric flow can be $\mathcal{O}\left(10^{4}\right)$ (e.g. Ref. [20]).

The most obvious place to investigate high Reynolds number turbulence is the atmosphere, where no particular effort has to be made to generate a very high Reynolds number flow. However, the flow is known to be unsteady and influenced by rotation and stratification. To escape boundary layer effects and the influence of the terrain, measurements have to be performed at great heights, typically using tall measurement towers in flat parts of the earth (e.g. [26], measuring at mountain research stations [27, 28], or with measurement balloons [29]. Moreover, statistical averaging over long periods is difficult due to possibly changing flow states. The recent availability of easy-to-operate unmanned aerial vehicles has opened the interesting possiblity of spatially sampling the velocity field of large areas in a relatively short time, i.e. in an 


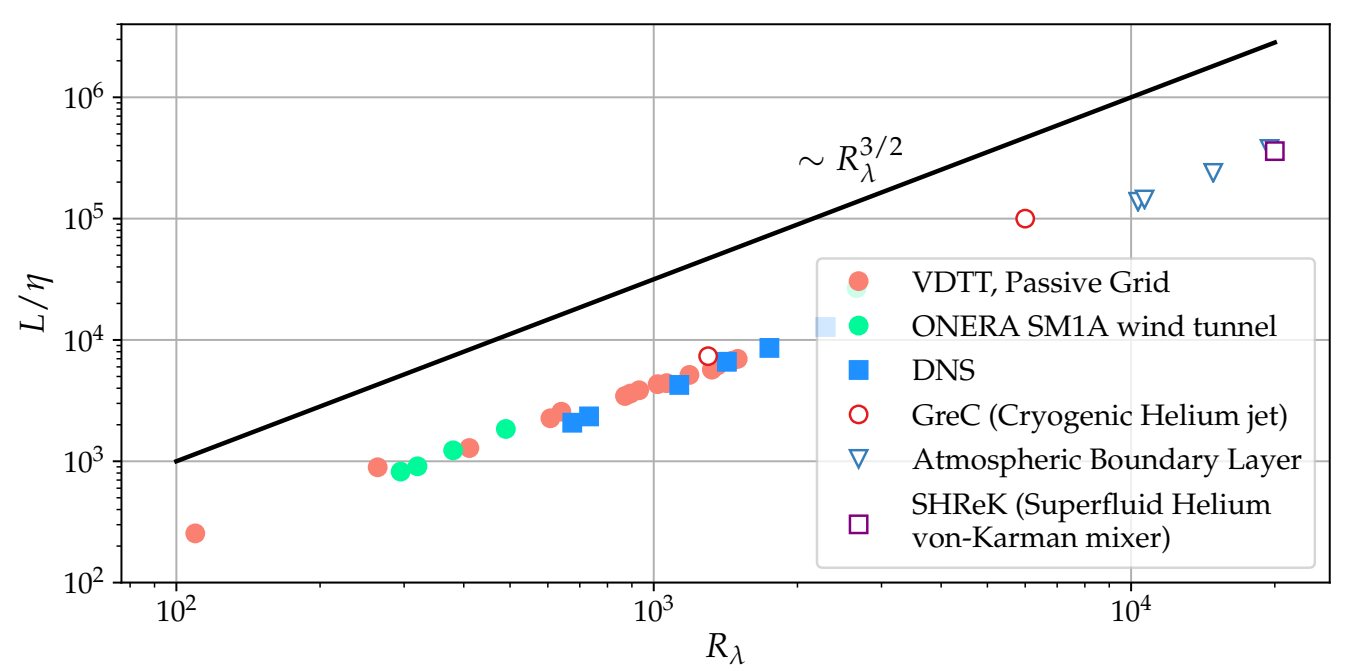

Figure 1.1: Separation of scales vs. Reynolds number for selected high-Reynolds number experiments. $L$ is the typical length scales of the largest flow structure, while $\eta$ is the length scale of viscous dissipation (see Ch. 3.3 for details). Flows with severe anisotropies and inhomogeneities are plotted in open symbols. VDTT data from [21], ONERA data from [22, 23], SHReK data derived from information in [24], GReC data derived from information in [25], Atmospheric data from [20].

approximately constant flow state [30].

Direct numerical simulations (DNS), i.e. the numerical integration of the Navier-Stokes-Equations (2.5) usually in a periodic box forced at particular wavenumbers, strongly depend on the availability of supercomputers. Even with the most advanced supercomputers, one must compromise between the Reynolds number, the temporal and spatial resolution of the simulation, and the duration of the simulation [31]. The largest Reynolds number $\left(R_{\lambda}=2297\right)$ in a DNS was obtained by Ishihara et al. [32], compromising small scale resolution and statistical fidelity. A better resolved DNS at $R_{\lambda} \approx 1300$ was obtained by P.K. Yeung and coworkers [31]. Even though the computing power needed for a DNS scales unfavourably with $R_{\lambda}^{18 / 4}$, numerical simulations will reach larger and larger values in the future and have come a long way since the first DNS by Orszag \& Patterson [33].

In pipe flows, extremely large Reynolds numbers are achieved in the Princeton Superpipe, which operates with air at almost 200 bars [34]. Pipe flows are in general wall-bounded flows, i.e. dominated by contributions of the flow boundaries, which inject turbulent kinetic energy through friction. They are therefore strongly influenced by mean shear and anisotropies. Wall-bounded flows are very common in nature and engineering and their understanding is of great importance. However, pipe flows do not feature a homogeneous, isotropic region and are therefore not well suited to study the nonlinear, iner- 
tial transport of energy.

When using experimental apparati of small dimensions, high $R_{\lambda}$ can be obtained by using fluids of low kinematic viscosity $v$, which allows the existence of very small, low-energetic eddies. The extreme case is superfluid or ultra-cold helium. In the SHReK experiment, turbulence is created by counterrotating two disks (von-Kármán mixer) in helium between 1.6 and $4.5 \mathrm{~K}$, and $R_{\lambda} \sim 20000$ can be reached [24]. The GReC experiment at CERN is a low-temperature helium jet, which reaches $R_{\lambda} \sim 8000$. While creating among the highest Reynolds numbers without wall effects, the usage of ultra-cold or superfluid helium bears extreme practical challenges. The facilities are very costly to operate and difficult to maintain due to the lengthy process of cooling the gas. Moreover, the small viscosity causes the smallest flow features to be smaller than $10 \mu \mathrm{m}$, which is a considerable technological challenge to measure [35].

Experiments operating in air at atmospheric pressures have to be very large to obtain large Reynolds numbers in free stream turbulence or must feature specialised forcing mechanisms. Such experiments are typically wind tunnels. A historical review of wind tunnels can be found in Ref. [36], this section concentrates on three air wind tunnels. First, the wind tunnel at Cornell University achieved $R_{\lambda} \sim 1000$ using an active grid and a detailed study of its small-scale turbulence has been performed [37]. The wind tunnel at the University of Oldenburg contains a $3 \times 3 \mathrm{~m}$ large active grid as well as the possibility to influence the largest flow scales by individually controlling its four fans. Using specialised active grid turbulence generation [37] and additionally modulating the rotation frequency of the fans, they reported $R_{\lambda} \sim 16000$ [38]. Given that the longitudinal correlation length of the velocity fluctuations was about $30 \times$ the size of the tunnel height, large flow inhomogeneities can be expected. Their tunnel is however ideal to recreate realistic inflow conditions for wind energy devices in a laboratory, i.e. similar to the unsteady turbulence in the atmosphere. Finally, the return flow of the commercial ONERA wind tunnel has delivered one of the most cited datasets on high Reynolds number turbulence [22] $\left(R_{\lambda} \sim 2500\right)$. Its operation is however extremely expensive (500 ooo Euros per week of operation), since up to 88 MW are applied on the fan shaft [39].

The state of turbulence research at the highest Reynolds numbers is roughly summarised in Fig. 1.1. (Approximately) homogeneous and isotropic turbulence studies exist up to a $R_{\lambda} \sim 2000$, but only very few datasets exist for $R_{\lambda}>1000$. Data at larger $R_{\lambda}$ are even more scarce and to the authorś knowl- 
edge subject to substantial inhomogeneities and anisotropy in all cases.

This thesis is concerned with another experiment explicitly aimed at creating high $R_{\lambda}$ under (approximately) homogeneous and isotropic conditions. The VDTT combines two of the approaches mentioned above to create a flow at $R_{\lambda} \lesssim 6000$ in a wind tunnel that easily fits into a relatively small experimental hall, can be operated at moderate costs, and whose flow properties are such that high-resolution measurements with state-of-the-art equipment are still possible. The fact that its flow properties can also be very finely tuned both at large and small scales makes it perhaps the single experiment most ideally suited for the study of turbulent flows worldwide at the time of writing. The experiment and its flow properties are described in detail in $\mathrm{Ch}$. 4 .

\subsection{This Thesis}

The main purpose of this thesis is to establish precision measurements at high $R_{\lambda}$ in the Max Planck Variable Density Turbulence Tunnel (VDTT). After a brief introduction of the necessary theoretical background in fluid dynamics and statistics of turbulence, as well as existing phenomenological models, the flow conditions in the VDTT in its configuration at the point of writing are discussed. In the following part, results from hot wire measurements are reported. To this end, first the technique is introduced emphasising procedures employed to optimise and verify hot wire anemometry in the specific environment of the VDTT. In the following chapters, new insights into high$R_{\lambda}$ turbulence obtained using this technique are presented to complete Part II. Part III introduces a setup capable of performing Lagrangian Particle Tracking in the VDTT. The thesis is concluded by a Discussion and an Outlook. 


\section{Chapter 2}

\section{The Equations of Motion}

This chapter will introduce the fundamental equations that govern turbulence dynamics largely following Davidson [40], Monin \& Yaglom [41], and Pope [42]. For details and more thorough derivations, the reader is kindly referred to those references.

\subsection{The Navier-Stokes Equations}

To derive the most fundamental equations of incompressible fluid mechanics, Newton's Second Law is applied to an infinitesimal fluid element of volume $V$ and density $\rho$ to yield the equation of motion in terms of the velocity field $\mathbf{u}$. The relevant forces are due to pressure and shear stresses. The pressure $P$ creates a force $-P \mathbf{A}$, which acts on all surfaces $d \mathbf{A}$ and yields a net force of

$$
F_{P}=\oint-P d \mathbf{A}=\int-\nabla P d V
$$

Here, the surface integral has been replaced by a volume integral by means of the Gauss theorem. The viscous forces are induced by the molecular coupling of the fluid element to its direct neighbours. Such internal forces per area are known as stresses described by the stress tensor $\tau_{i j}$. It denotes the component $i$ of the viscous stresses acting on the surface labeled $j$. In a Newtonian fluid, which shall be assumed here, the shear stress depends on the fluid density $\rho$, its kinematic viscosity $v$, and the velocity gradients.

$$
\tau_{i j}=\rho v\left(\frac{\partial u_{i}}{\partial x_{j}}+\frac{\partial u_{j}}{\partial x_{i}}\right) \equiv 2 \rho v S_{i j}
$$

In passing, the strain-rate tensor $S_{i j}$ has been introduced for convenience. The net force per unit volume in direction $i$ due to the stresses at the surfaces is 
then given by the gradients of these stresses in all $j$ directions:

$$
f_{i}=\frac{\partial \tau_{i j}}{\partial x_{j}}
$$

Here, as well as in the remainder of this thesis we use the Einstein summation convention that repeated indices are summed over.

In the absence of external forces (e.g. gravity), the forces on a fluid parcel are entirely due to the presence of neighbouring fluid parcels. Similarly, the kinematics these forces induce contain a term due to the immersion of the fluid parcel in a larger flow field. For example, the flow profile of a stationary pipe flow $(\partial u / \partial t=0$ everywhere) depends on the radial distance $r$ from the pipe centerline. Therefore, a fluid parcel that is displaced in the radial direction will experience a change in $\mathbf{u}$ proportional to the local radial gradient $\partial \mathbf{u} / \partial r$. In general, the change in $\mathbf{u}$ can be due to a temporal change $\partial u / \partial t$ or an advection towards a region of different flow speeds $\mathbf{u} \nabla \mathbf{u}$. This is commonly summarised into the convective derivative ${ }^{1}$

$$
\frac{D u_{i}}{D t}=\frac{\partial u_{i}}{\partial t}+u_{j} \frac{\partial u_{i}}{\partial x_{j}} .
$$

Combining eqs. (2.1), (2.2), and (2.4) one arrives at the balance of forces

$$
\rho\left(\frac{\partial u_{i}}{\partial t}+u_{j} \frac{\partial u_{i}}{\partial x_{j}}\right)=-\frac{\partial P}{\partial x_{i}}+v \frac{\partial^{2} u_{i}}{\partial x_{j} \partial x_{j}} .
$$

In addition to these dynamical equations, the conservation of mass

$$
\partial\left(\rho u_{i}\right) / \partial x_{i}=\partial \rho / \partial t
$$

is required. In the case of an incompressible fluid, i.e. when $\partial \rho / \partial t=0$, this reduces to

$$
\nabla \cdot \mathbf{u}=0
$$

Eqs. (2.5) and (2.6) form the incompressible Navier-Stokes equations of a Newtonian fluid. They are nonlinear, coupled differential equations. Their complexity is mainly due to the nonlinear term $u_{j}\left(\partial u_{i} / \partial x_{j}\right)$ and the nonlocal pressure term (see following section). It is therefore most interesting to study situations where those terms dominate the dynamics. This is the case when

\footnotetext{
${ }^{1}$ In the Lagrangian framework, introduced in Sec. 3.4.1 the convective derivative results naturally from deriving the equation of motion in the comoving coordinate system of the fluid parcel
} 
the viscous term is small, i.e. when the Reynolds number is high. In this regime the properties of turbulence are expected to be universal. This makes the study of high Reynolds number turbulence important from a theoretical point of view.

It is important to mention that in most flows additional forces act on the infinitesimal fluid volume as a whole - so called body forces. For example, in the case of the atmosphere the coriolis forces play an essential role in the turbulence dynamics, and in numerical simulations particular wavenumbers are excited by an idealised forcing term. As shall be shown in the next section, a localised external force will have a global impact mediated by the infinite propagation speed of pressure waves in an incompressible medium.

\subsection{Nonlocality of the Pressure Term}

Upon application of the divergence to the Navier-Stokes equations (2.5) one obtains after considering the incompressibility result (2.6),

$$
\frac{\partial^{2}(P / \rho)}{\partial x_{i}^{2}}=-\frac{\partial}{\partial x_{i}}\left(u_{j} \frac{\partial u_{i}}{\partial x_{j}}\right)
$$

The solution of this equation is the Biot-Savart law [40]:

$$
P\left(x_{i}\right)=\frac{\rho}{4 \pi} \int \frac{\frac{\partial}{\partial x_{i}}\left(u_{j} \frac{\partial u_{i}}{\partial x_{j}}\right)}{\left|x_{i}-x_{i}^{\prime}\right|} d x_{i}^{\prime}
$$

One can now insert eq (2.8) into (2.5) and arrive at a closed equation for the velocity field $\mathbf{u}$. The equations got however even more difficult to treat analytically, because they are not only nonlinear, but also nonlocal. Physically, this means that any disturbance or flow structure at a point $x_{i}^{\prime}$ influences all other points $x_{i}$ in the entire flow field. In an incompressible fluid this influence happens instantaneously.

\subsection{The Energy Equation and Dissipation}

The forces acting on the infinitesimal fluid element we consider here, perform work, which will be dissipated into random molecular motion, i.e. heat. To obtain the energy equation, we multiply eq. (2.5) by $u_{i}$ and expand the 
viscous term.

$$
\frac{\partial \mathbf{u}^{2} / 2}{\partial t}=-\frac{\partial}{\partial x_{i}}\left(\mathbf{u}^{2} / 2\right) u_{i}-\frac{\partial}{\partial x_{i}}\left(\frac{P}{\rho}\right) u_{i}+\frac{\partial}{\partial x_{i}} u_{j} \tau_{i j} / \rho-2 v S_{i j} S_{i j}
$$

This is an evolution equation for the kinetic energy density inside the fluid parcel. The terms on the right-hand side are as follows:

- convection of kinetic energy across the boundary,

- deformation of the boundary due to pressure forces,

- deformation of the boundary due to viscous coupling to adjacent fluid parcels,

- conversion of kinetic energy into heat.

The last term is of great importance, because it is the only global energy sink of the system (except for work done on the flow boundaries, often also in the form of heat). Thus, turbulence converts kinetic energy into heat at a dissipation rate

$$
\varepsilon=\left\langle 2 v S_{i j} S_{i j}\right\rangle=2 v\left\langle\frac{\partial u_{i}}{\partial x_{j}} \frac{\partial u_{i}}{\partial x_{j}}\right\rangle
$$

$\langle\cdot\rangle$ denotes a spatial average unless stated otherwise. The strain-rate tensor is given by

$$
S_{i j}=\frac{1}{2}\left(\frac{\partial u_{i}}{\partial x_{j}}+\frac{\partial u_{j}}{\partial x_{i}}\right) .
$$

Because of the Second Law of Thermodynamics, this heat cannot be transformed back into the structured fluid motion of turbulence. Its role as a well-defined, unique sink of energy puts the dissipation rate in the center of many turbulence theories encompassing all turbulence length scales. If the turbulence is homogeneous, and isotropic [41]

$$
\varepsilon=15 v\left\langle\left(\frac{\partial u_{1}}{\partial x_{1}}\right)^{2}\right\rangle
$$

This definition will be used throughout Part II to estimate $\varepsilon$ from one-dimensional velocity measurements.

In spectral space, eq. (2.12) reads by the derivative property of the fourier transform

$$
\varepsilon=2 v \int_{0}^{\infty} \mathbf{k}^{2} E(\mathbf{k}) d \mathbf{k}=15 v \int_{0}^{\infty} k_{1}^{2} E_{11}\left(k_{1}\right) d k_{1}
$$


Again, the second equality is only valid in homogeneous, isotropic turbulence.

\subsection{Vorticity}

The presence of rotational motions has been identified as a defining feature of turbulent flows ever since the experiments of Reynolds [3]. This is quantified by the vorticity of the flow defined as

$$
\boldsymbol{\omega}=\nabla \times \mathbf{u} .
$$

Using the vector calculus identity

$$
(\mathbf{u} \cdot \nabla) \cdot \mathbf{u}=\nabla\left(\frac{u^{2}}{2}\right)-\mathbf{u} \times \omega
$$

the Navier-Stokes equation (2.5) can be rewritten as

$$
\partial \mathbf{u} / \partial t=\mathbf{u} \times \boldsymbol{\omega}-\frac{\nabla P}{\rho}-\nabla u^{2} / 2+v \nabla^{2} \mathbf{u} .
$$

Note that this equation is in principle Bernoulli's equation for a stationary, irrotational flow in an inviscid fluid. Taking the curl along with a version of the aforementioned vector identity yields the vorticity equation

$$
\frac{\partial \boldsymbol{\omega}}{\partial t}+(\mathbf{u} \cdot \nabla) \boldsymbol{\omega}=(\boldsymbol{\omega} \cdot \nabla) \mathbf{u}+v \nabla^{2} \boldsymbol{\omega}
$$

In analogy to the energy equation (2.9), the square of the vorticity - called enstrophy - is introduced. Its equation of motion reads [40]

$$
\frac{D\left(\omega_{i} \omega_{i}\right) / 2}{D t}=\omega_{i} \omega_{j} S_{i j}-v \varepsilon_{i j k} \varepsilon_{i l m} \frac{\partial \omega_{k}}{\partial x_{j}} \frac{\partial \omega_{m}}{\partial x_{l}}+v \frac{\partial}{\partial x_{l}}\left(\varepsilon_{l m n} \omega_{m} \varepsilon_{n j k} \frac{\partial \omega_{k}}{\partial x_{j}}\right)
$$

The velocity gradients can be decomposed into a rotational and a potential component:

$$
\frac{\partial u_{i}}{\partial x_{j}}=\frac{1}{2}\left(\frac{\partial u_{i}}{\partial x_{j}}+\frac{\partial u_{j}}{\partial x_{i}}\right)+\frac{1}{2}\left(\frac{\partial u_{i}}{\partial x_{j}}-\frac{\partial u_{j}}{\partial x_{i}}\right)=S_{i j}-\frac{\epsilon_{i j k} \omega_{k}}{2}
$$

where $\epsilon_{i j k}$ is the Levi-Civita symbol. Therefore, the velocity gradients are the combined result of strain and vorticity. 
Through the derivative property of the Fourier transform, $\left\langle\boldsymbol{\omega}^{2}\right\rangle$ can be expressed in terms of the energy spectrum of the velocity fluctuations (see Ref. [41] for details):

$$
\left\langle\omega^{2}\right\rangle=\int_{0}^{\infty} k^{2} E(k) d k
$$

Upon comparison of (2.20) with (2.13), the correspondence between enstrophy and dissipation becomes evident:

$$
\varepsilon=v\left\langle\omega^{2}\right\rangle
$$




\section{Chapter 3}

\section{The Statistics of Homogeneous Isotropic Turbulence}

\subsection{Three Statistical Objects}

The purpose of this section is to define the three most important statistical objects that will be studied for the remainder of this thesis, namely the correlation function, the energy spectrum, and the structure functions.

\subsubsection{The Correlation Function}

The correlation function of a velocity field $u_{i}(\mathbf{x})$ is defined as

$$
C_{i j}(\mathbf{r})=\left\langle u_{i}(\mathbf{x}) u_{j}(\mathbf{x}+\mathbf{r})\right\rangle
$$

Unless denoted otherwise, $\langle\cdot\rangle$ denotes an average over the flow field. In the case of a statistically isotropic field, the correlation function is independent of the direction of $\mathbf{r}$. We can therefore consider the correlation function (and the other two statistics introduced here) in a coordinate system, where one axis $\left(x_{1}\right)$ is parallel to $\mathbf{r}$ and the other two perpendicular. In this case, only the diagonal components are nonzero and $C_{22}=C_{33} \neq C_{11}$ [41]. I.e. there are only two components that need to be considered here: the longitudinal correlation function $C_{11}$ (also called $C_{L L}$ ) and the transverse correlation function $C_{22}=C_{33} \equiv C_{N N}$, where the velocity components are normal to $\mathbf{r}$. In summary:

$$
C_{11}(r)=C_{L L}(r)=\left\langle\left(\mathbf{u}(\mathbf{x}) \cdot \mathbf{e}_{\mathbf{r}}\right)\left(\mathbf{u}(\mathbf{x}+\mathbf{r}) \cdot \mathbf{e}_{\mathbf{r}}\right)\right\rangle,
$$




$$
C_{22}(r)=C_{33}(r)=C_{N N}(r)=\left\langle\left(\mathbf{u}(\mathbf{x}) \times \mathbf{e}_{\mathbf{r}}\right)\left(\mathbf{u}(\mathbf{x}+\mathbf{r}) \times \mathbf{e}_{\mathbf{r}}\right)\right\rangle,
$$

where $\mathbf{e}_{\mathbf{r}}$ denotes the unit vector in the direction of the increment vector $\mathbf{r}$. Since both the energy spectrum and structure function can be derived from ( $n$ th order) correlation functions, similar simplifications exist for these statistical objects as well.

\subsubsection{The Energy Spectrum}

The energy spectrum tensor is the fourier transform of the correlation function $[41]$ :

$$
\phi_{i j}(\mathbf{k})=\frac{1}{(2 \pi)^{3}} \int C_{i j}(\mathbf{r}) e^{-i \mathbf{k r}} d \mathbf{r} .
$$

In the case of an incompressible fluid, we know that $k_{i} \phi_{i j}=k_{j} \phi_{i j}=0$. In combination with the simplifications through isotropy outlined in the previous section, the energy spectrum of homogeneuos, isotropic turbulence in an incompressible fluid can be expressed by a single function $E(k)$ :

$$
\phi_{i j}=\frac{E(k)}{4 \pi k^{2}}\left(\delta_{i j}-\frac{k_{i} k_{j}}{k^{2}}\right) .
$$

In Part II of this thesis, the results from hot wire measurements will be discussed. The only component of the energy spectrum tensor accessible to such measurements is $\phi_{11}\left(k_{1}\right)$, also denoted as $E_{11}\left(k_{1}\right)$. In terms of the energy spectrum function

$$
E_{11}\left(k_{1}\right)=\int_{0}^{k_{1}} \frac{E(k)}{k}\left(1-\frac{k_{1}^{2}}{k^{2}}\right) d k
$$

\subsubsection{Structure Functions}

The statistical information about a phenomenon can be described by a probability distribution function (PDF). Since in turbulent flows structures of different scales interact with each other, the scale-by-scale statistics are of prime interest. To combine the two approaches, one traditionally measures the $n$-th order velocity increment statistics, also called structure functions

$$
S_{n}^{i}(\mathbf{r})=\left\langle\left(u_{i}(\mathbf{x}+\mathbf{r})-u_{i}(\mathbf{x})\right)^{n}\right\rangle .
$$

$S_{n}^{i}$ and the $n$-th order correlation function $\left\langle\left(u_{i}(\mathbf{x}+\mathbf{r}) u_{i}(\mathbf{x})\right)^{n}\right\rangle$ are trivially 
connected. Therefore, just as correlation functions, $S_{n}^{i}$ can be fully decomposed into a component in the direction of $\mathbf{r}$ (longitudinal) and a component normal to $\mathbf{r}$ (transverse). Since we consider longitudinal structure functions in most cases, we call the longitudinal structure function of order $n$

$$
S_{n}(r)=\left\langle\left(u_{x}(x+r)-u_{x}(x)\right)^{n}\right\rangle
$$

Thus, structure functions exhibit similar properties in terms of complexity as correlation functions and energy spectra. They can in fact be converted into each other. The structure function is however easier to interpret at higher orders than correlation functions or higher-order spectra. Further, the structure function is smoother than the energy spectrum, because it is an average of local quantities (the velocity increments), whereas the energy spectrum implicitly assumes the existence of some periodicity, which is in general not present. For this reason, structure functions can be considered a very simple wavelet transform [43] with a wavelet consisting of two $\delta$-distributions.

\subsection{The Karman-Howarth-Equation}

After introducing the canonical tools of statistical analysis of turbulence, we merge the definitions from the previous section with the fundamental fluid dynamics equations from Sec. 2.1. For this, we consider the NavierStokes-Equations (2.5) for the velocity field $u_{i}$ and the velocity field shifted by an increment $r$ called $u_{i}^{\prime}$. Following Davidson [40],

$$
\frac{\partial u_{i}}{\partial t}=u_{i} \frac{\partial\left(u_{k}\right)}{\partial x_{k}}-\frac{\partial(p / \rho)}{\partial x_{i}}+2 v \frac{\partial^{2}}{\partial x_{i} \partial x_{j}} u_{i}
$$

An equivalent equation can be written down for the shifted velocity field $u_{j}^{\prime}\left(x_{k}^{\prime}\right)$. We now multiply equation (3.9) by $u_{j}^{\prime}$, add it to the equivalent equation for $u_{j}^{\prime}$ and average. Assuming isotropy, one arrives at

$$
\frac{\partial\left\langle u_{i} u_{j}^{\prime}\right\rangle}{\partial t}=-\frac{\partial}{\partial r_{k}}\left(\left\langle u_{i} u_{k} u_{j}^{\prime}\right\rangle+\left\langle u_{i} u_{j}^{\prime} u_{k}^{\prime}\right\rangle\right)+2 v \frac{\partial^{2}}{\partial r_{k}^{2}}\left\langle u_{i} u_{j}^{\prime}\right\rangle
$$

with $r_{k}=x_{k}-x_{k}^{\prime}$.

This equation is easier to interpret when written in terms of the normalised longitudinal correlation functions $f(r)=C_{11}(r) / u_{R M S}^{2}$ and $K(r)=$ 


$$
\begin{aligned}
\left\langle u(x)^{2} u(x+r)\right\rangle / u_{R M S}^{3}: & \\
\frac{\partial}{\partial t} u^{2} f(r, t) & =\frac{1}{r^{4}} \frac{\partial}{\partial r}\left(r^{4} u^{3} K(r)\right)+2 u \frac{1}{r^{4}} \frac{\partial}{\partial r}\left(r^{4} u^{2} \frac{\partial f}{\partial r}\right) .
\end{aligned}
$$

We have arrived at an evolution equation for the second-order longitudinal correlation function in isotropic turbulence (removing the simplification of isotropy yields a longer, but otherwise similar equation, see e.g. [44, 41, 45]). However, this equation has two unknowns: The second- and third order correlation functions $f(r)$, and $K(r)$, respectively. This is the closure problem of turbulence arising in the equations of turbulence statistics. It is a major addition to the mathematical complexity of the problem. To circumvent the closure problems, several closure assumptions have been suggested. For example, the quasi-normal approximation assumes the fourth-order statistics to be Gaussian even at small increments [46]. This is not only contradicting experimental data (see Sec. 3.3.4), but also leads to serious theoretical problems. For example, $E(k)<0$ in an important range of wavenumbers [47]. Some of these inconsistencies are removed by the eddy-damped quasi-normal approximation, where an additional time-irreversibility is installed by adding an eddy-viscosity term. More detailed views on closure schemes can be found in e.g. $[48,40]$.

Eq. 3.11 can be recast in terms of the structure functions $S_{2}(r)$ and $S_{3}(r)$. It is straightforward to see that $S_{2}(r)=2 u^{2}(1-f(r))$, and $S_{3}(r)=6 u^{3} K(r)$. With this one arrives at

$$
-\frac{2}{3} \varepsilon-\frac{1}{2} \frac{\partial S_{2}(r)}{\partial t}=\frac{1}{6 r^{4}} \frac{\partial r^{4} S_{3}(r)}{\partial r}-\frac{v}{r^{4}} \frac{\partial}{\partial r}\left(r^{4} \frac{\partial}{\partial r} S_{2}(r)\right)
$$

Evidently, the third order structure function is completely determined by the time- and scale dependence of $S_{2} . S_{3}$ is unique in this sense, because the evolution equations of higher orders contain additional pressure contributions. The terms in eq. (3.12) correspond to different ranges of scales. The second term on the left hand side comes from the statistical nonstationarity, e.g. due to the decay of turbulent kinetic energy. The last term on the right hand side describes the effects of viscosity on $S_{3}$.

In a statistically stationary flow $\partial S_{2} / \partial t=0^{1}$. In the limit of very large $R_{\lambda}$,

\footnotetext{
${ }^{1}$ Statistical stationarity means that averaged quantities are constant throughout one instance of the flow. It makes no statement about the repeatability of such a flow.
} 
$v \rightarrow 0$. In this case, eq. (3.12) can be integrated over $r$ leaving [49]

$$
S_{3}(r)=\frac{4}{5} \varepsilon r
$$

Hence, at those scales in a stationary flow, where viscous effects (and any large-scale forcing) are negligible, the third order statistics are uniquely determined by the dissipation rate $\varepsilon$. This range of scales is called the inertial range. In Sec. 3.3 it will become apparent that (3.13) is only a special case of a hierarchy of scaling laws for the $n$-th order structure function, albeit the only one that can be derived rigorously from the equations of motion.

This section closes by the conversion of (3.11) into spectral space. The application of a Fourier transform yields

$$
\frac{\partial}{\partial t} E(k, t)=T(k, t)-2 v k^{2} E(k, t)
$$

where the spectrum of scale-by-scale energy transfer $T(k, t)$ has been introduced.

\subsection{Phenomenology and Scaling}

\subsubsection{The Cascade Picture}

Already Leonardo da Vinci must have realised the multiscale organisation of turbulent flow in his studies of water leaving a rectangular channel [50]. Richardson [51] was the first to hypothesise the existence of an energy cascade from large scales to smaller ones. In particular, he envisioned turbulence as a sea of vortex structures ("whorls" or "eddies"), that subsequently break up into smaller and smaller eddies "and so on to viscosity (in the molecular sense)".

As outlined in Sec. 1.1, the presence of turbulence is immediately recognisable when presented with a sufficiently visualised turbulent flow. This is likely owing to the presence of easily recognisable, unique structures. Just as turbulence itself, these structures are difficult to capture in a strict, mathematical sense. In the literature (and this thesis) such structures are referred to as "eddies". They can be loosely described as (weakly) coherent fluid motion with a length scale $\ell$.

The cascade picture of turbulence - in particular in its original form envisioned by Richardson [51] - makes frequent use of this picture. In the Richardson picture of turbulence, a turbulent flow originates from eddies of size $\ell_{1}$, 
that carry the majority of the turbulent kinetic energy. These eddies break up into a number of smaller eddies of size $\ell_{2}$, which in turn break up into even smaller eddies. The cascade ends when the energy within each eddy can be directly dissipated into heat.

This picture has been very influential for the turbulence research it preceded, but its strict hierarchy of eddies breaking up into smaller eddies has proven inaccurate. In particular, these eddies coexist at many different length scales and interact with each other nonlinearly. This is reflected in the two different approaches to turbulence. In the Eulerian framework one or more snapshots of the fluid field are considered and scale-by-scale statistics emerge. Because Eulerian measurements are relatively straightforward to realise, this has been the major way to study turbulence and the vast majority of statistical theories is centered around spatially resolved flow fields.

The insufficiencies of the strict Richardson cascade picture are even more obvious when considering the motion of a single fluid parcel over time. It shall be argued in Sec. 3.4.1 that this Lagrangian framework is equivalent to the Eulerian and in some sense a more natural way to study turbulence (cf. $[52,53])$. We observe that fluid parcels regularly experience extreme accelerations suggesting a small-scale motion followed by relatively quiescent periods, which cannot be captured by the simple Richardson cascade model. Such measurements are however extremely difficult and the underlying phenomenology is not nearly as well-developed as in the Eulerian case.

We have thus introduced the length scale $\ell$ of an eddy, which can be supplemented by an eddy time scale (eddy turnover time) $\tau_{\ell}$, and an eddy velocity scale $u_{\ell}=\ell / \tau_{\ell}$.

This phenomenological model implicitly introduced a far-reaching assumption, namely that the power introduced at the beginning of the cascade (the large scale $L$ ) and the power ultimately dissipated into heat are connected by a simple factor. The power present at any scale $\ell$ can be defined by dimensional analysis as $u_{\ell}^{3} / \ell$. In the particular case of the energy injection scale $L$ the relevant velocity is the root mean square (RMS) of the velocity fluctuations. The power dissipated into heat is given by the dissipation rate $\varepsilon \sim v\left\langle\left(\partial u_{i} / \partial x_{i}\right)^{2}\right\rangle$. The cascade picture implies that

$$
\varepsilon=C_{\varepsilon} \frac{u^{3}}{L}
$$

This relation is frequently called the "Zeroth Law" of turbulence. The value of $C_{\varepsilon}$ depends on the precise definition of $L$, and the flow geometry or forcing 
scheme, but is constant at large Reynolds numbers. Eq. 3.15 has particular implications for the limit of $\operatorname{Re} \rightarrow \infty$, which can be seen as $v \rightarrow 0$ with $L=$ const. If this relation holds as $v$ becomes smaller and smaller, the velocity gradients $\partial u_{i} / \partial x_{i}$ must become steeper and steeper, ultimately causing singularities. This is called the dissipation anomaly and is discussed in greater detail in Sec. 7.1.

\subsubsection{Self-Similarity}

The previous section has made clear that the velocity field of a turbulent flow cannot be smooth in the limit of infinite $R_{\lambda}$. Indeed, O. Reynolds observed in his groundbreaking experiments [3] described in Sec. 1.1 that as soon as the energy input exceeded a certain threshold, the smooth flow broke down into a flow with steep velocity gradients efficiently mixing the dye he used to visualize it. The flow apparently changed its geometry dramatically, because the kinetic energy could not be dissipated efficiently enough by the gradients of a smooth velocity field. The system then forms a fractal to accomodate the excess energy input giving it a non-smooth character. Such phenomena are prevalent in numerous physical and biological systems, such as metabolism, fractures, and geology. They typically exhibit fractal or selfsimilar features (see [54, 55] for more examples). As Jiménez [56] writes: "It may be said that the reason why fractals, geometric or otherwise, are prevalent in nature is the need of handling solicitations that cannot be managed by smooth means.".

A defining feature of fractals is their self-similarity, i.e. its features $f$ at a scale $\ell$ appear again in a scaled version of themselves when observing it at a scale $\lambda \ell$. Mathematically, a scalar field $f(r)$ is called self-similar if [54]

$$
f(\ell)=\lambda^{\zeta} f(\lambda \ell)
$$

for all $\lambda>0$. The scaling exponent $\zeta$ is an important characteristic of the fractal properties of the system.

\subsubsection{Kolmogorov's 1941 Theory}

In 1941, Kolmogorov published his seminal work that would influence generations of researchers $[57,49]$. It is commonly abbreviated as $\mathrm{K}_{41}$. It combines the Richardson conjecture of an energy cascade [51] and the concept of self-similarity. The work is centered around three hypotheses and 
two definitions regarding the statistics of velocity increments $\mathbf{u}(\mathbf{x}+\mathbf{r})-\mathbf{u}(\mathbf{x})$. Those statements are paraphrased in the following.

Definition of Local Homogeneity and Isotropy A flow is locally homogeneous and isotropic in a region of the flow, if the statistics of velocity differences are invariant under translations (homogeneity), rotations and reflections (isotropy) over that domain.

Hypothesis of Local Isotropy and Homogeneity The velocity difference statistics are homogeneous and isotropic over a region of the flow's typical length scale $L$, time scale $T=L / U$, and sufficiently far away from the flow boundaries.

First Similarity Hypothesis The statistics of the small-scale $(r<L)$ velocity differences in locally isotropic turbulence depend only on the dissipation rate $\varepsilon$ and the kinematic viscosity $v$.

Second Similarity Hypothesis If $L \gg r \gg \eta$, where $\eta$ is the typical length scale of viscous dissipation, the velocity increment statistics depend on the dissipation rate $\varepsilon$ only and are independent of viscosity.

These hypotheses have far-reaching consequences. First, they allow to quantify the typical length scales of the flow. The size of smallest length scale in the flow (the size of the smallest eddies) is proportional ${ }^{2}$ to the Kolmogorov length $\eta$. According to the first similarity hypothesis, $\eta$ can only depend on $\varepsilon$ and $v$. The only dimensionally correct length scale that can be formed by these two quantities is

$$
\eta=\left(\frac{v^{3}}{\varepsilon}\right)^{1 / 4} .
$$

Similarily, a time- and velocity scale of the dissipative regime can be formed:

$$
\begin{aligned}
\tau_{\eta} & =\sqrt{v / \varepsilon} \\
u_{\eta} & =(\varepsilon v)^{1 / 4} .
\end{aligned}
$$

The size of the largest length scale over which local isotropy can be assumed according to $\mathrm{K}_{41} \mathrm{I}$ is less well defined. A common estimate is the integral over the correlation function $C_{11}(r)$ starting from $r=0$. The upper

\footnotetext{
${ }^{2}$ Note that Kolmogorov never claimed equality. In fact, the smallest eddies are likely roughly of size $\approx 10 \eta[58]$.
} 

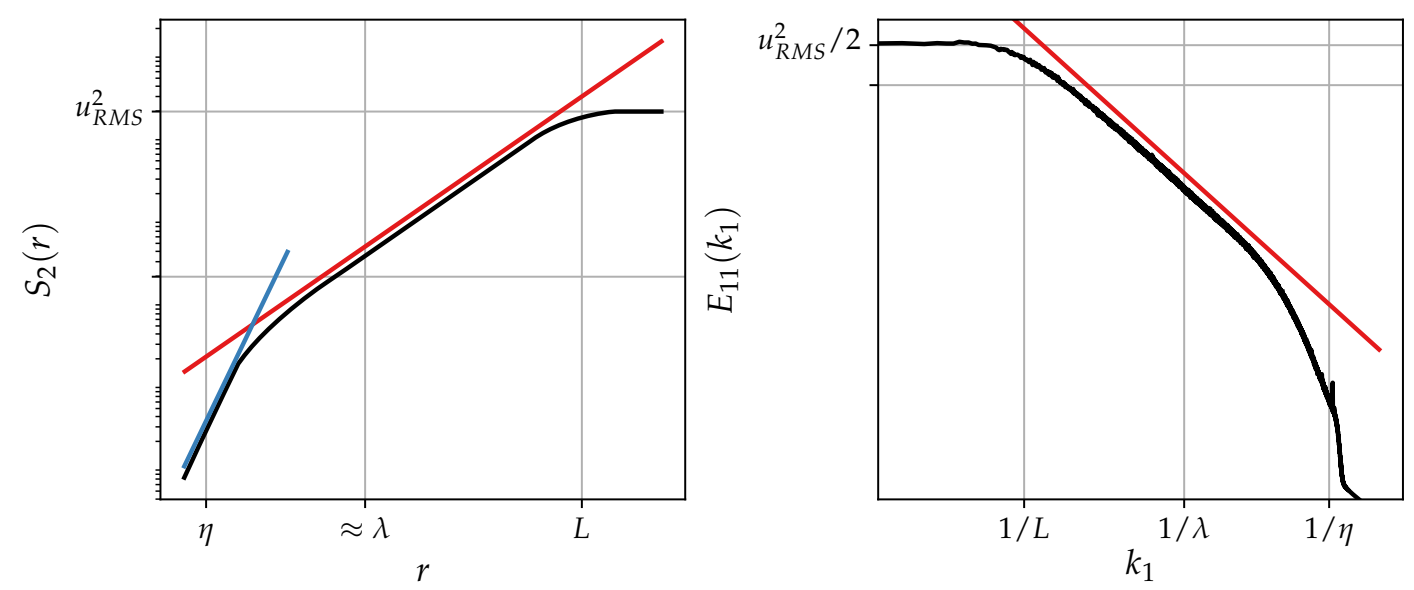

Figure 3.1: Left: Illustration of the second order structure function $S_{2}(r)$. Blue line: smallscale scaling $\sim r^{2}$, red line: $\mathrm{K}_{41}$ inertial range scaling $\sim r^{2 / 3} . S_{n}(r)$ is constant at the largest scales. Right: Measurement of the one-dimensional energy spectrum. Red line: K41 inertial range prediction $\sim k^{-5 / 3}$. At large scales, $E_{11}=$ const., whereas $E(k<1 / L) \sim k^{\alpha}$ with $2 \leq$ $\alpha \leq 4$. The large-scale behaviour of $E(k)$ is not recovered in the one-dimensional surrogate due to the integral in eq (3.6).

boundary is often taken as the first point where $C_{11}\left(r_{0}\right)=0$, but other definitions are possible $[59,60]$.

There exists another important length scale first defined by Taylor [18]. The Taylor scale $\lambda$ is originally defined over the correlation function as well $[18,42]$, but the more practical definition

$$
\lambda=\sqrt{\frac{u^{2}}{(\partial u / \partial x)^{2}}}=\sqrt{\frac{15 v u^{2}}{\varepsilon}}
$$

is presented here. It is of great importance for fundamental turbulence studies, as the commonly used Taylor-scale Reynolds number is based on this length scale. However, its interpretation is not as clear as in the case of $\eta$, or $L$. $\lambda$ was originally meant to characterise the scale at which dissipative effects become negligible [18]. It has been observed in the past that the average distance between zero-crossings of the fluctuation signal is very close to $\lambda$ [19], and that $\lambda$ is linked to the onset of Markovian properties in the turbulent cascade [61].

$\mathrm{K}_{41}$ augments the phenomenology of the Richardson cascade picture of turbulence by a quantification of the most important length- and time scales involved. Kolmogorov's theory further makes detailed predictions about how the velocity increment statistics scale with the increment $r$. The $n$-th order structure function defined by (3.8) has units of $(\mathrm{m} / \mathrm{s})^{n}$. A family of function that fulfils the self-similarity condition (3.16) are power laws of the form 
$f(r) \sim r^{\zeta}$. Assuming a self-similar power law for $S_{n}(r)$, the only dimensionally correct result with $\varepsilon$ as the only free parameter is

$$
S_{n}(r) \sim(\varepsilon r)^{n / 3}
$$

Note that for $n=3$, the scaling is readily confirmed by the 4/5-law derived directly by averaging the Navier-Stokes equations. This lends support to the ad-hoc assumption of a power law.

From this the following overall picture emerges for $S_{n}$ : At very small scales

$r \lesssim \eta$, where viscosity dominates the dynamics, $S_{n} \sim r^{2}$ as can be seen from a Taylor expansion around $r=0$. For very large separations $r>L$, the velocity field is decorrelated and $S_{n}(r) \sim u_{R M S}^{n}$. If those two regimes are separated well enough, a third distinct range of scales emerges, where nonlinear dynamics (inertia) dominate. Here, viscosity and flow geometry play only a minor role and the structure function follows eq. (3.21).

Through equivalent arguments, Kolmogorov could predict the scaling of the energy spectrum in the inertial range

$$
E\left(\eta^{-1} \ll k \ll L^{-1}\right)=C_{K} \varepsilon^{2 / 3} k^{-5 / 3} .
$$

These results have far-reaching consequences. They imply that in a statistical sense only the largest scales are flow dependent. The statistics of the small scales (including but not limited to the dissipation scales) do not depend on their origin, i.e. are universally shared among different types of flow up to a constant prefactor. It turns out that even the prefactors are at least similar from flow to flow.

In the decades following its original publication, the spirit of the $\mathrm{K}_{41}$ predictions has been confirmed in experiments too numerous to list. At leading order, $\mathrm{K}_{41} \mathrm{i}$ is a remarkably good description of turbulence statistics, in particular given its simple derivation.

It should be noted that the history of these predictions is much richer than suggested here. In fact, Prandtl [62], Onsager [63], and Heisenberg [64] and Weizsäcker [52] arrived at the same conclusions independently.

\subsubsection{Intermittency}

The $\mathrm{K}_{41}$ framework was a milestone in the understanding of turbulence statistics. However, it was quickly realised $[65,66]$ that $\varepsilon$ is an intermittent function of space and time, and therefore a simple average is an oversim- 


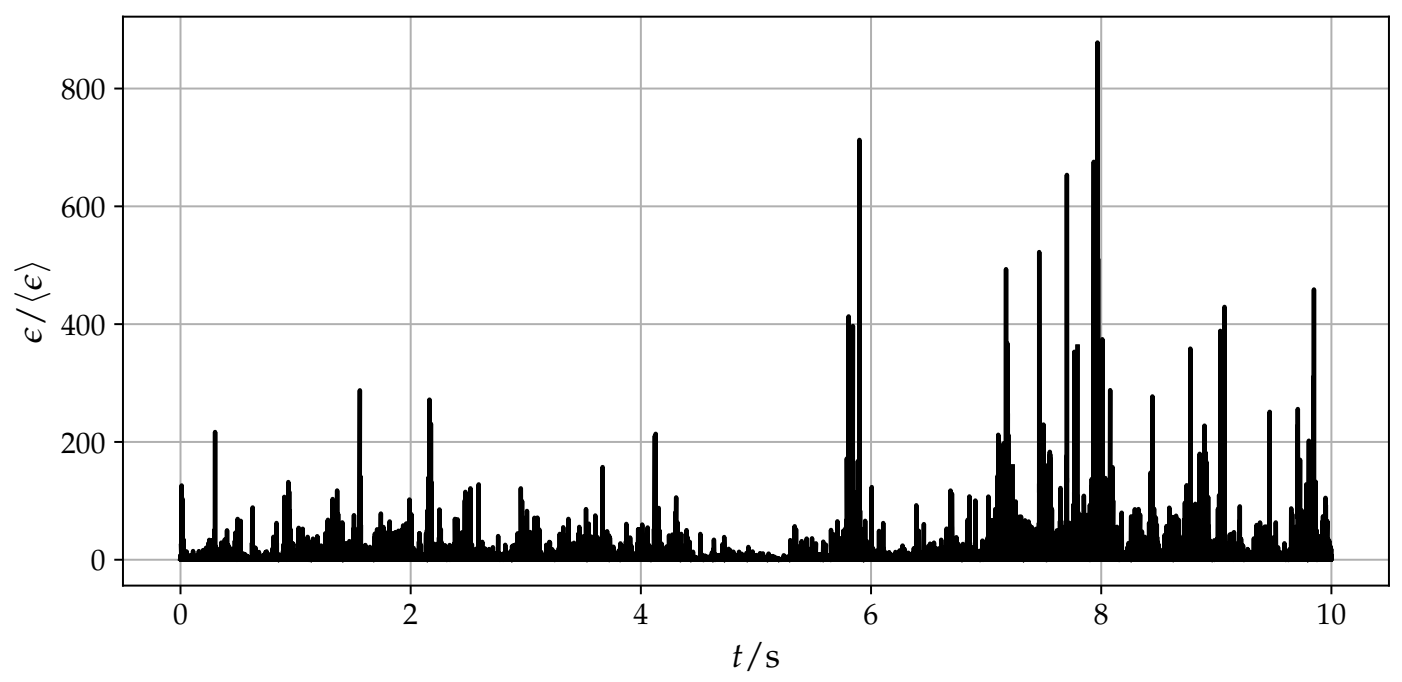

Figure 3.2: The time series of the dissipation rate $\left(R_{\lambda} \approx 4000\right)$ computed from the a single velocity component illustrating the intermittency of $\varepsilon$. The signal is an extremely spiky function of time (and space) regularly taking values several hundred times its mean.

plification. Fig. 3.2 illustrates that the dissipation rate frequently departs extremely far from its average values. This objection has led to the development of several theories that aim at modelling this intermittency. Historically, Kolmogorov quickly refined his original theory [66] assuming a probability distribution that accounts for the abundance of extreme dissipation values. Such a distribution is the log-normal distribution, which causes corrections to the structure function scaling exponents of the form

$$
\zeta_{n}=\frac{n}{3}-\frac{\mu}{18} n(n-3)
$$

$\mu$ is the intensity of the intermittency.

Different assumptions for the PDF of $\varepsilon$ have been proposed. For example, Andrews et al. [67] assume a Gamma-Distribution for $\varepsilon$.

Another probability-based model is the Log-Poisson model by Dubrulle [68]. It yields

$$
\zeta_{n}=(1-\Delta) n / 3+\frac{\Delta\left(1-\beta^{n / 3}\right)}{1-\beta} .
$$

$\beta$ is equivalent to $\mu$ in eq. (3.23), and $\Delta$ is proportional to the codimension of the "most intermittent dissipative structures" [68]. These structures are explicitly captured by the largest statistical moments, since higher moments emphasise the tails of the distribution. An intuitive assumption for the nature of these structures are extremely thin vortex filaments, which are practically one-dimensional. For this special case of eq (3.24) one gets $\beta=\Delta=2 / 3$. This 
is known as the She-Leveque model [69], which precedes the Dubrulle model historically. Incidentially, this yields an acceptable fit to measured values of $\zeta_{n}$ without the need for free parameters (see Fig. 7.6, and e.g. Ref. [70])) and with a plausible physical interpretation. The two models furthermore predict a order-by-order hierarchy of scaling laws, i.e. $S_{m} \sim S_{n}$. This is strikingly similar to the empirical observation that $S_{n}$ scales with $S_{|m|}$ over a much longer range of scales than $S_{n}(r)$ (known as "Extended Self-Similarity" (ESS), see Chs. 6 and 8 [71]. Finally, the Dubrulle model is a limiting case of the random $\beta$ model[72].

The random $\beta$ model belongs to a class of models that are based on the geometric interpretation of intermittency $[72,73]$. Intermittency of $\varepsilon$ implies that certain regions of space contain large values of $\varepsilon$, whereas others do not. A way to construct such a spatial distribution is to start at a scale $\ell_{0}$ (in turbulence: the length scale, where the energy is injected into the cascade) and to subdivide the volume $\ell_{0}^{3}$ into smaller volumes of size $\left(\ell_{0} / 2\right)^{3}$ (or any other factor $>0$ ). A fraction $\beta$ of these subvolumes carry "active" eddies (strong dissipation). These "active" subvolumes are subsequently divided in $N$ steps into even smaller subvolumes of scale $\ell_{0} / s^{N}$, such that the energy transfer at the $N$-th iteration is $\varepsilon_{N}=\beta^{N} u_{n}^{3} / \ell_{N}$. Here, $u_{N}$ is the RMS velocity within all active subvolumes at scale $\ell_{N}$. This yields structure function scaling exponents

$$
\zeta_{n}=\frac{n}{3}+(3-D)\left(1-\frac{n}{3}\right)
$$

$D$ can then be interpreted as the fractal dimension of the active eddy volume. According to eq. (3.25), the structure function scaling exponents are still a linear function of $n$. While measurements of $\zeta_{n}$ are subject to large uncertainties at high $n$, they generally point towards a nonlinear behaviour of $\zeta_{n}$ [74]. However, when looking at the closed-loop integral over the velocity field instead of velocity differences, the available data points towards a combination of two linear functions valid for different ranges of $n$ [75]. This would correspond to a bifractal model, where the dissipation can exist on two different fractal sets, where the measured scaling exponent is the smaller of the two at any given order $n$. The next step is naturally to assume the existence of infinitely many fractal sets with scaling exponents between $\left(h_{\min }, h_{\max }\right)$, which is known as the multifractal model $[76,77,78,72]$. An example of such a model is the random $\beta$-model [72], where the fraction $\beta$ occupied by active eddies (strong dissipation) is an independent random variable for each subvolume and cascade step. Together with the arguments made for the $\beta$-model, the 
random $\beta$ model yields for the structure function scaling exponents

$$
\zeta_{n}=\frac{n}{3}-\log _{2}\left(\langle\beta\rangle^{1-n / 3}\right)
$$

\subsection{Lagrangian Turbulence}

\subsubsection{The Lagrangian Framework}

The preceding chapters of this thesis have been exclusively concerned with the Eulerian description of the flow field, i.e. the vector field of the flow velocity $\mathbf{u}(\mathbf{x}, t)$. The corresponding statistical objects are based on observations of that flow field at one or two locations in the flow. There exists however a different, but equivalent method of describing fluid flow. Instead of considering the fluid parcel at each point in the volume, we label each fluid parcel by its location $\mathbf{X}$ at the point $t=0$ by $\mathbf{x}_{\mathbf{0}}$ and track its position as a function of time.

The relation between this Lagrangian description in a comoving reference frame and the Eulerian description in the laboratory frame is straightforward [54]:

$$
\begin{aligned}
\frac{d \mathbf{X}\left(\mathbf{x}_{\mathbf{0}}, t\right)}{d t} & =\mathbf{u}\left(\mathbf{X}\left(\mathbf{x}_{\mathbf{0}}, t\right)\right) \\
\mathbf{X}\left(\mathbf{x}_{\mathbf{0}}, t\right) & =\mathbf{x}_{\mathbf{0}}
\end{aligned}
$$

Similarily, the Lagrangian velocity is defined as

$$
\mathbf{U}\left(\mathbf{x}_{\mathbf{0}}, t\right)=\frac{d}{d t} \mathbf{X}\left(\mathbf{x}_{\mathbf{0}}, t\right)=\mathbf{u}\left(\mathbf{X}\left(\mathbf{x}_{\mathbf{0}}, t\right)\right)
$$

Writing down the Navier-Stokes equations in an entirely Lagrangian framework yields complicated shear-stress terms and is of little insight [79]. Here, only the implicit version using the Eulerian equations shall be presented [54]:

$$
\frac{d}{d t} \mathbf{U}\left(\mathbf{x}_{\mathbf{0}}, t\right)=\left.\left[-\nabla P(\mathbf{x}, t)+v \nabla^{2} \mathbf{u}(\mathbf{x}, t)\right]\right|_{\mathbf{x}=\mathbf{X}\left(\mathbf{x}_{0}, t\right)} .
$$

The nonlinearity of the advective gradient has been absorbed into the Lagrangian acceleration. Therefore, measurements of the acceleration of a fluid particle contain direct information about the nonlinear facets of turbulence. The Lagrangian description is furthermore of great practical importance, since transport and mixing processes are more intuitively understood in a comov- 
ing reference frame [8o]. However, wind tunnel experiments of transport phenomena, such as two-particle dispersion, are a practical challenge. These phenomena require particle tracking over long periods of time $>50 \tau_{\eta}$, such that a stationary measurement device would need to resolve a very large field of view sufficiently or the device needs to be moved with the mean flow. The dispersion characteristics of turbulence are beyond the scope of this thesis.

\subsubsection{Lagrangian Structure Functions}

In principle, all statistical objects defined in Sec. 3.1 exist in the Lagrangian reference frame with spatial increments usually replaced by temporal increments along the trajectory of a fluid parcel. However, quantities like the energy spectrum rely on unbiased sampling, i.e. a constant sampling independent of the value under study. Faster particles cross any finite measurement volume in a shorter time than slower ones, which introduces a sampling bias. Since structure functions are local in scale and space, they are less plagued by preferential sampling.

The $n$-th order structure function is defined as the $n$-th order moment of the velocity increments separated by a time step $\tau$ along the trajectory of a fluid element:

$$
S_{n}^{L}(\tau)=\left\langle\left(u_{i}(t+\tau)-u_{i}(t)\right)^{n}\right\rangle
$$

Equivalent to the Eulerian Framework, the $\mathrm{K}_{41}$ theory yields for the inertial range of scales

$$
S_{n}^{L}(\tau)=C_{n}(\varepsilon \tau)^{n / 2},
$$

which is straightforward to derive by dimensionally matching $\tau$ and $\varepsilon$ under the assumption of self-similarity. If the inertial range statistics take a universal form at larger $R_{\lambda}, C_{n}$ should approach the same constant for every homogenous, isotropic turbulent flow. In the case of $n=2$ (typically called $C_{0}$ ), this constant is closely connected to the Richardson constant of pair dispersion for separations in the inertial range and the structure functions of passive scalars [81]. It furthermore appears in relative dispersion- and transport models. The experimental measurement of $C_{0}$ is however very difficult. In general, Lagrangian statistics of adequate precision have been accessible for little more than two decades at the point of writing [82, 83] with some earlier atmospheric measurements being subject to large uncertainties [84]. This is a very short time frame compared to the body of Eulerian measurements acquired over more than one century. The measurement of inertial range quantities 
in the Lagrangian reference frame is particularly difficult, because its inertial range grows by a factor $R_{\lambda}^{1 / 2}$ slower than the Eulerian [85]. The existing measurements and numerical simulation arrive at values of $2<C_{0}<9$ with very small or absent inertial range scalings.

As $\tau \rightarrow 0$, a Taylor expansion yields that $S_{n}^{L}\left(\tau \ll \tau_{\eta}\right) \sim \tau^{n}$. Using in addition, Kolmogorov's First Hypothesis of Similarity along with dimensional arguments,

$$
S_{2}\left(\tau \ll \tau_{\eta}\right)=a_{0} \sqrt{\frac{\varepsilon^{3}}{v}} \tau^{2} .
$$

$a_{0}$ is called the normalised acceleration variance. It is predicted to approach a universal constant in the limit of very large $R_{\lambda}$ (cf. Sec. 3.4.3).

Note that in the Lagrangian framework a straightforward relation between statistics of different orders such as the Kármán-Howarth equation (3.12) does not exist. Therefore, the scaling laws (3.32) are only based on dimensional ar-

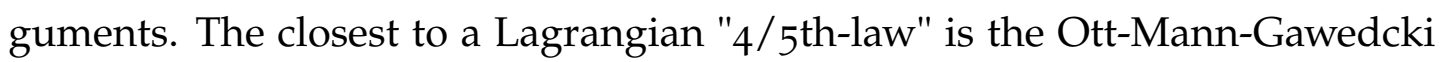
relation $[86,87,88]$. Considering two particles with velocities $\mathbf{v}_{\mathbf{1}}$ and $\mathbf{v}_{\mathbf{2}}$ and an initial separation in the inertial range,

$$
\left\langle\left.\frac{d}{d t}\left(\mathbf{v}_{\mathbf{1}}(t)-\mathbf{v}_{\mathbf{2}}(t)\right)^{2}\right|_{t=0}\right\rangle=-4 \varepsilon
$$

This is intimately connected with the time-irreversiblity of turbulence [89].

\subsubsection{Lagrangian Accelerations}

The Lagrangian acceleration is a fundamental quantity in the study of turbulent flows, since it is equivalent to the convective derivative as demonstrated in Sec. 3.4.1. It is furthermore a direct measure of the smallest scales and its magnitude scales with $\ell^{-1 / 3}$ in the inertial range, i.e. fluid element accelerations become smaller with increasing length scale.

Heisenberg [64] and Yaglom [53] derived a relation for the acceleration variance based on Kolmogorov's K 41 self-similarity theory:

$$
\left\langle a^{2}\right\rangle=a_{0} \varepsilon^{3 / 2} v^{-1 / 2}
$$

In the presence of small-scale intermittency, $\left\langle a^{2}\right\rangle$ might carry a Reynolds number dependence. For example, if the dissipation rate is log-normally distributed, $\left\langle a^{2}\right\rangle \sim R_{\lambda}^{9 \mu / 16}$ [9o]. Since the measurement of accelerations at high $R_{\lambda}$ requires extremely well-resolved spatiotemporal coordinates of the fluid parcel locations, its statistics are accessible in experiments only relatively re- 
cently $[83,91,90]$.

Since the Lagrangian acceleration directly probes the small-scale structure of the flow, its probability distribution function (PDF) has to reflect the relative abundance of extreme events. In other words, the PDF of accelerations must be heavy-tailed. This has been confirmed in DNS [92] and experiments [91]. 


\section{Chapter 4}

\section{Experimental Setup and Flow Properties}

The purpose of this chapter is twofold: First, the experimental facility in which the experiments were performed is described. Particular attention is paid to the active grid that allowed the creation of higher $R_{\lambda}$ and is the most recent addition to the wind tunnel structure. Then, the large-scale flow properties are discussed in three brief sections. The corresponding measurements are the hot wire measurements tabulated in Appendix unless stated otherwise.

\subsection{The Variable Density Turbulence Tunnel}

The Variable Density Turbulence Tunnel (VDTT) is a closed-loop highpressure wind tunnel. It is described in detail by Bodenschatz et al. [36]. In the following a brief description of the facility is provided for the reader's convenience. The wind tunnel consists of two parallel, horizontal $11.68 \mathrm{~m}$ long tubes with an inner diameter of $1.84 \mathrm{~m}$ connected by two elbows of inner diameter $1.52 \mathrm{~m}$. The centerlines of the tubes are $3.5 \mathrm{~m}$ apart. The total volume is $\approx 88 \mathrm{~m}^{3}$ enclosed by $20 \mathrm{~mm}$ thick steel ( $18 \mathrm{~mm}$ in the elbows). The pressure vessel is approved for operation with sulphur-hexaflouride $\left(\mathrm{SF}_{6}\right)$ up to 15 bar between 15 and $30^{\circ} \mathrm{C}$.

The gas is propelled by a $210 \mathrm{~kW}$ electric motor, which is located at one end of the lower straight tube. The motor rotates a fan with 20 blades at up to $24 \mathrm{~Hz}$ accelerating the working fluid to mean flow speeds between 0.5 and $5.5 \mathrm{~m} / \mathrm{s}$. It is water cooled with the cooling water provided by the institute cooling system through a dedicated water line into the tunnel. 


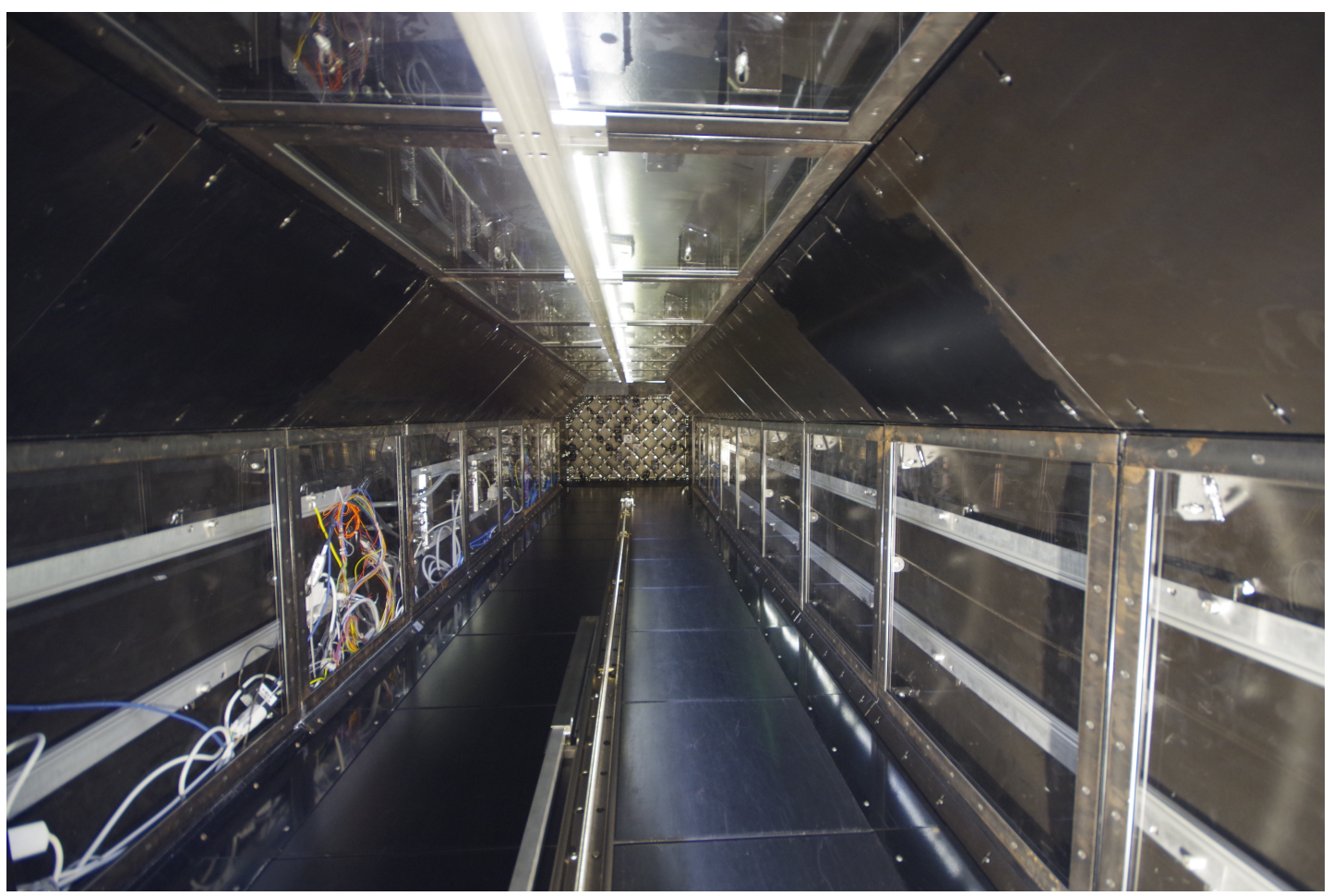

Figure 4.1: Upper measurement section looking upstream with the active grid at the end.

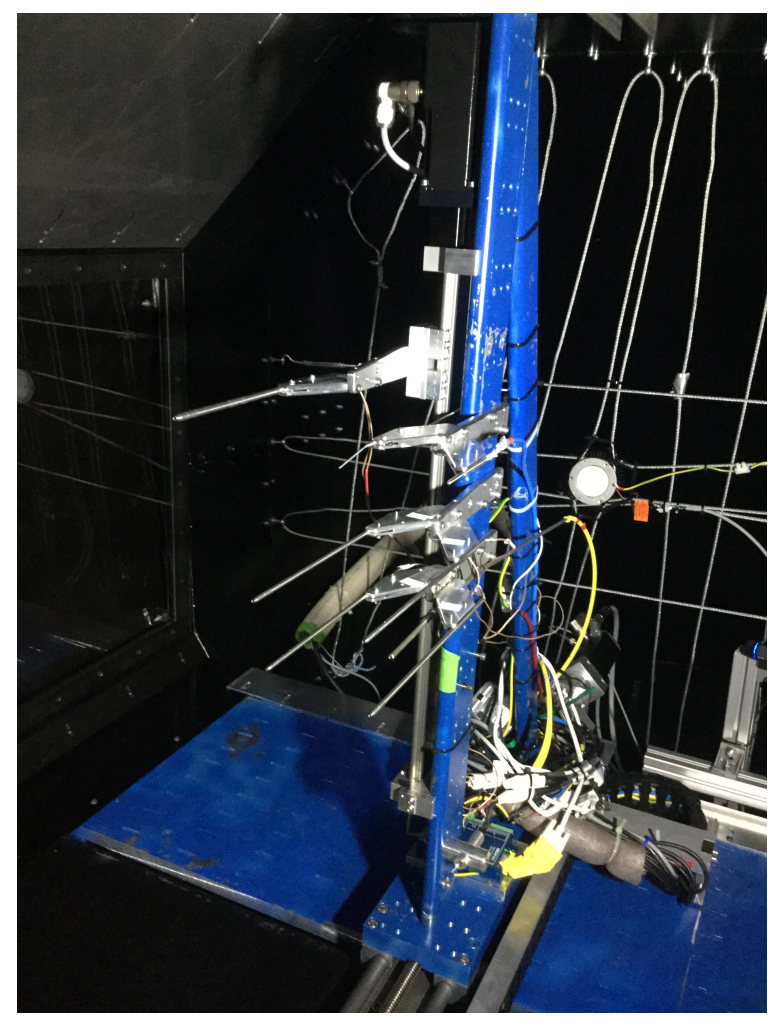

Figure 4.2: Vertical traverse with hot wire holders and pitot tubes installed looking downstream. A vertically mounted linear stage on the left side can be used to record velocity profiles 
The operating gas first flows through one elbow, which is immediately followed by a $240 \mathrm{~kW}$ heat exchanger at the beginning of the upper straight cylindrical tube. This heat exchanger removes the heat injected into the flow by the electrical motor once it has dissipated into heat. The heat exchanger consists of two registers of water-cooled plates that are stacked in such a way that cool water flows from both the top and the bottom of the cross-section to avoid a temperature gradient along the tunnel height. The cooling water supply is isolated from the pressurised tunnel inside and the laboratory environment. Leaks of $\mathrm{SF}_{6}$ into the cooling water as well as cooling water leaks into the $\mathrm{SF}_{6}$ would be detected. The rectangular heat exchanger is smoothly adapted to the cylindrical cross section through a contraction and subsequently adapted to the approximately octangular cross section shape of the following measurement section by an expansion. During the expansion the flow encounters three meshes of increasing mesh spacing (0.850mm, 1.267mm, 2.833mm). Together with the heat exchanger slots these meshes laminarise the flow and destroy any residual mean vorticity introduced by the rotating fan. At the beginning of the upper measurement section turbulence production mechanisms are installed. The measurements in this thesis were carried out using an active grid (see Sec. 4.2), in previous experiments a passive grid was installed $[93,94,95]$.

After the active grid the flow passes through another vertical expansion from $104 \mathrm{~cm}$ to $116 \mathrm{~cm}$ height and is then left to develop freely within the boundaries of the $8.8 \mathrm{~m}$ long, $1.5 \mathrm{~m}$ wide, and $1.17 \mathrm{~m}$ high (measured at the downstream end) measurement section. Note that the section was elevated by $12 \mathrm{~cm}$ compared to the setup described in Ref. [36]. The section features a traverse that can be moved in the streamwise and vertical directions. A cable guide brings digital connections, RG223 coaxial signalling cables, as well as plastic pipes to the traverse, which provide the basis for hot wire measurements. Ch. 10 describes the implementation of a camera platform that is about $6 \mathrm{~m}$ downstream from the active grid. After leaving the upper measurement section the flow is guided through the other elbow through another set of three meshes before entering the lower measurement section. This measurement section was not in use during the experiments presented here. After this section the fluid enters the fan again. 


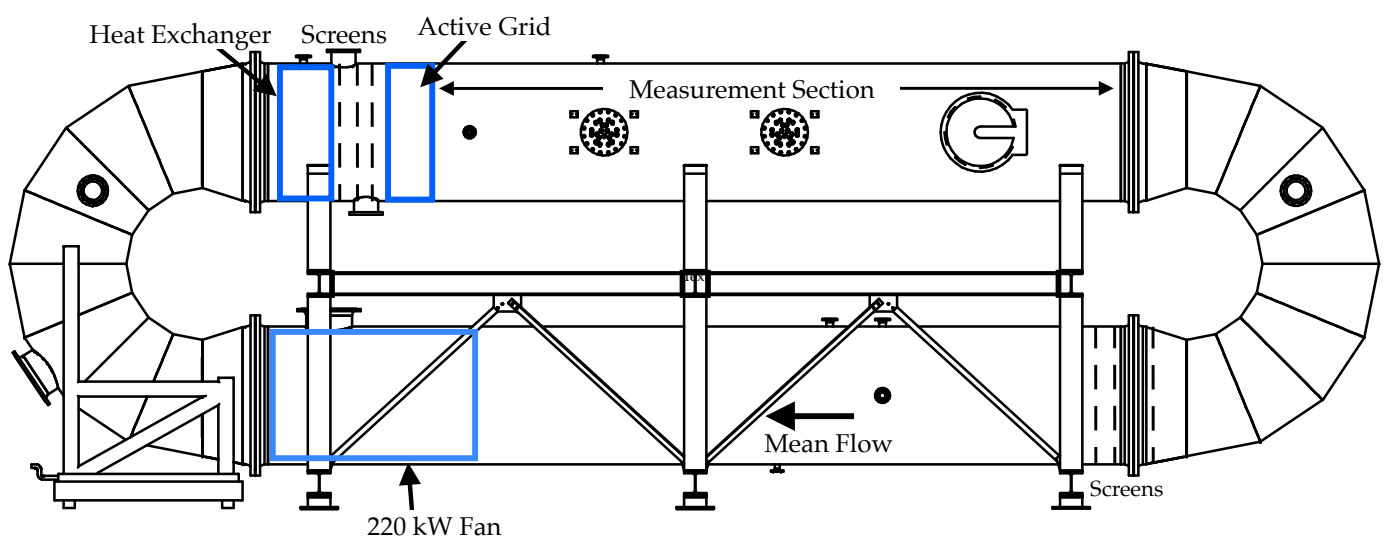

Figure 4.3: Side view of the Max Planck Variable Density Turbulence Tunnel indicating essential parts of the flow. Graphic by A. Kubitzek.

\subsection{The Active Grid}

The most classic turbulence experiment is perhaps the wind tunnel flow in the far-field wake of a solid grid of regularly spaced bars [96]. This flow is very close to the idealised case of homogeneous, isotropic turbulence [97]. It also has a rather low turbulence intensity, which allows the application of Taylor's frozen flow hypothesis (see Sec. 5.2, Ref. [98]) to convert single-location time series into one-dimensional flow fields. The energy injection scale $L$ is well-defined by the spacing $D$ of the grid bars. While these properties make it a very good choice to study fundamental aspects of decaying turbulence, they also bring difficulties: The Reynolds number $R_{\lambda}$ depends on the RMS of the velocity fluctuations $u$. Thus, when the turbulence intensity $u / U$ is low, a high mean flow velocity $U$ is required to obtain high Reynolds numbers. High mean flow velocities require very fast sensors (see Ch.5).

Further, the grid spacing $D$ is limited by the wind tunnel dimensions to maintain a high degree of homogeneity and isotropy. Therefore, the energy injection scale $L$ is limited to a fraction of the wind tunnel dimensions and difficult to adjust. The aim of the active grid in the VDTT is to create higher turbulence intensities and larger energy injection scales while sacrificing only little of the flow isotropy and homogeneity.

Makita \& Sassa [99] were the first to create an active grid and show that it produces turbulence that is comparable to the canonical passive grid turbulent flow. Their grid consisted of agitator winglets mounted to rotating rods. This setup and variations of it were the blueprint of most active grids built subsequently (see Ref. [100] for a review). Active grids with rotating rods were used to generate homogeneous, isotropic turbulence at high 

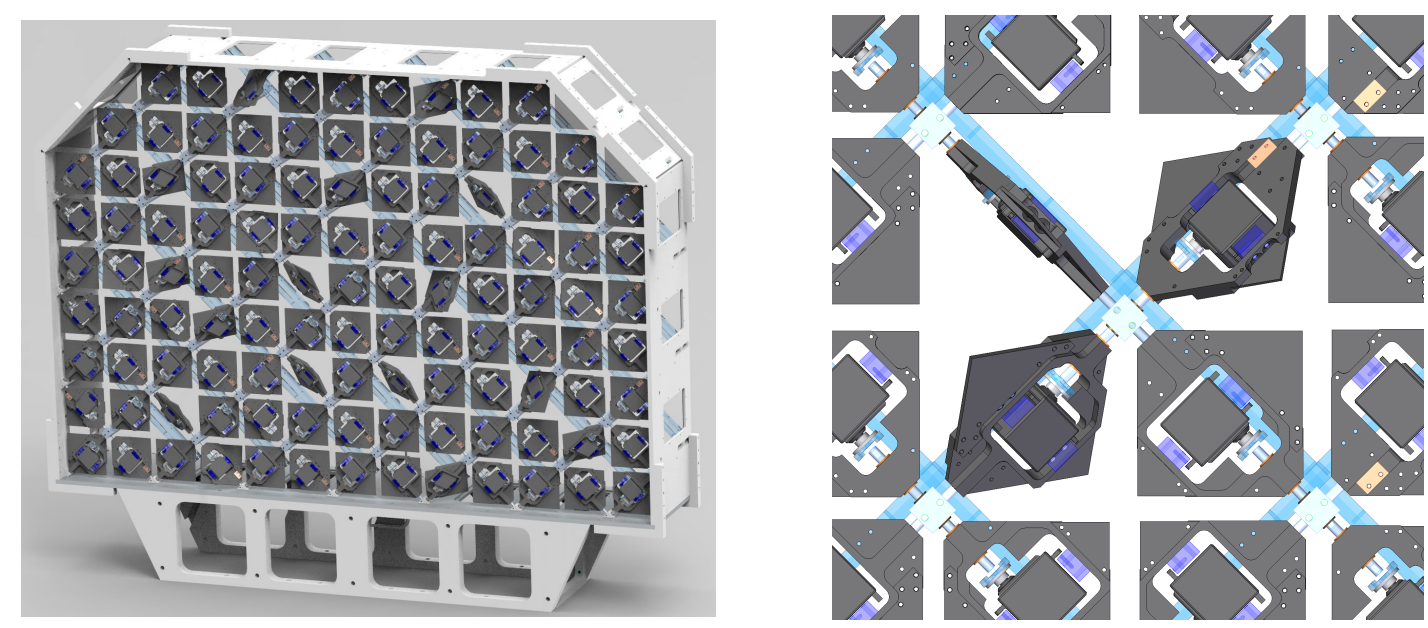

Figure 4.4: Left: Overview of active grid structure with most flaps in closed state. The outer aluminum structure is not exposed to the flow. Right: Close view on some active grid flaps with flaps pointing at (clockwise) $0^{\circ}, 45^{\circ}, 90^{\circ}$, and $-45^{\circ}$ with the flow pointing out of the plane. The center of each flap has a servo motor, which is decorated by plastic plates to form a $11 \mathrm{~cm}$ side length winglet. Graphic by A. Kubitzek, reprinted with permission from [108].

Reynolds numbers [101], generate conditions that closely resemble the atmospheric boundary layer [102, 103], create uniform velocity gradient [102], avoid or generate shear [104, 105], and to investigate wind turbines [106] and wind turbine arrays [107].

The active grid installed in the VDTT is a significant advancement over the Makita-style grids of rotating rods. It consists of 111 winglets that are completely independent, i.e. not connected by rods. Each $11 \mathrm{~cm} \times 11 \mathrm{~cm}$ square winglet has a Futaba BLS152 servo motor mounted in its center. This allows the winglet to rotate by $\pm 90 \mathrm{deg}$ from its open position at $0 \mathrm{deg}$ at a speed of $40^{\circ}$ per o.1s. In the open position, the winglet's blockage is minimal and one of their diagonals is parallel to the streamwise direction (see Fig. 4.4). At \pm 90 deg one side of the winglets directly faces the flow, respectively. The winglets are mounted in such a way that strong deflections of the flow are prevented and the turbulence is generated by the interaction of several wakes rather than large-scale vortices. The servo motors are powered by $1224 \mathrm{~V}$ power supplies within the tunnel and controlled by two SD84 boards connected to a computer via USB and Ethernet.

The blockage of the active grid to the fluid inertia can be up to practically $100 \%$. When filled with $\mathrm{SF}_{6}$ at 15 bars and the fan pushing fluid towards it this would correspond to $>8$ tons of fluid being pushed through the remaining slots between the flaps. This is not only a danger to the winglets, but comes close to the structural limits of the entire tunnel due to the sudden loss of 
angular momentum and the associated torque. Such a situation can occur either when commanded by the user or when for some reason the active grid motors receive no power or signal. The former case is prevented by the software that controls the grid (see below). When the total blockage exceeds $70 \%$, all angles are reduced by a common factor to bring the blockage below that threshold. The latter case of sudden loss of power or signal is even more dramatic, because the stable position of the flaps in the presence of flow is closed, i.e. blocking the flow. Thus, a loss of power leads to the sudden closure of the active grid flaps. To prevent damage to the grid and the tunnel, the active grid is constantly monitored and a loss of power or signal triggers an emergency stop of the wind tunnel fan.

The unique flexibility of the active grid allows for unseen ways to create turbulence, which has been shown in Ref. [109]. The authors use an active grid that is virtually identical to the one installed in the VDTT, but slightly larger. They have developed a protocol that drives the active grid and is described here briefly. It rests upon operations on a $N \times M \times T$-Matrix of grid angles $A_{m, n, t}$, where $n$ and $m$ are the coordinates of a virtual grid. Seven wingelts are padded to each dimension of the matrix to avoid boundary effects when computing correlations. The index $t$ corresponds to the order in which the angle configurations are realised on the active grid. The algorithm starts by creating a completely random instance of $A_{m, n, t}$. The matrix $A_{m, n, T / 2}$ is chosen as the first set of grid angles. The key step is to correlate $A_{m, n, T / 2}$ in all three dimensions before initiating the motion of the winglets on the active grid. The correlation function and its parameters determine the exact form of the forcing and have a big impact on the turbulence properties [109, 108]. To illustrate this we consider a top-hat correlation function $C_{T H}$ of correlation length $\sigma_{S}$ and -time $\sigma_{t}$ centered around the indices $(5,5, T / 2)$ :

$$
C_{T H}=I \begin{cases}1 & \text { for } 5-\sigma_{S}<m, n<5+\sigma_{S} \text { and } T / 2-\sigma_{t}<t<T / 2+\sigma_{t} \\ 0 & \text { else }\end{cases}
$$

$I$ is a normalisation constant. In this case an averaging over the $\sigma_{s}$ adjacent winglets in each direction and the values $A_{5,5, t}$ for $t=T / 2 \pm \sigma_{t}$ would take place and lead to the final angle for winglet coordinate $(5,5)$. This procedure is repeated for all values of $A_{m, n, T / 2}$ and the resulting matrix determines the next set of angle sent to the active grid. The matrix $A_{m, n, T / 2}$ is now updated with the correlated values. Finally, the matrix $A_{m, n, 1}$ is discarded, $A_{m, n, t} \rightarrow A_{m, n, t-1}$, and $A_{m, n, T}$ is a new, random $N x M$ matrix with values 


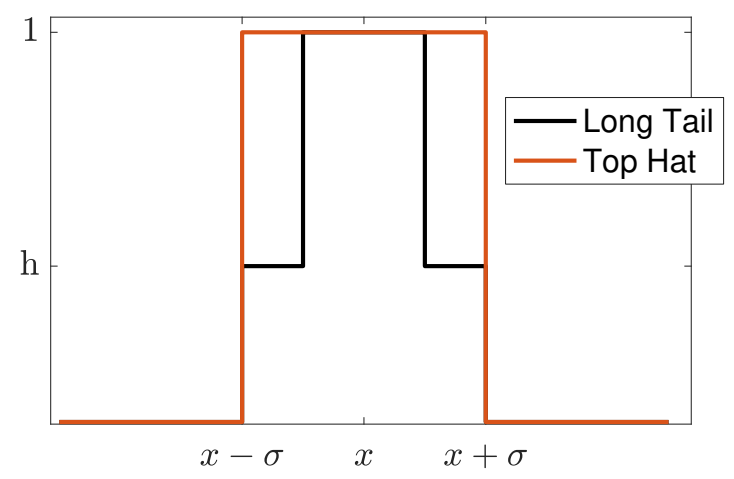

Figure 4.5: Long Tail and Top Hat correlation functions used to calculate the stream of correlated grid angles

between \pm 90 deg with a pre-defined RMS. This algorithm forms a random stream of grid angle sets that are correlated in space and time. $C_{T H}$ is only a simple, illustrative example, much more complex shapes of the correlation function are possible and the effects are described in detail in [109].

This thesis does not contain a detailed study of active grid protocols, but some experience on the effects of different grid protocols is communicated. The measurements presented here were taken exclusively using either a fully random mode with a pre-defined angle RMS, a top hat correlation function (TH), a long tail correlation function (LT) or a completely opened, stationary grid. The top hat and long tail correlation functions are sketched in Fig. 4.5. The grid protocols used are indicated by the following identifiers: Spatial Correlation Function $\sigma_{S} / 0.11 \mathrm{~m}$; Temporal Correlation Function $\sigma_{T} / 0.1 \mathrm{~s}$. For example, a grid protocol with a long tail spatial correlation with $\sigma_{S}=0.55 \mathrm{~m}$ and a top hat temporal correlation with $\sigma_{T}=0.3 \mathrm{~s}$ would be abbreviated as $\mathrm{LT}_{5} \mathrm{TH}_{3}$.

The active grid allows turbulent kinetic energy to be injected into the flow with unseen flexibility and opens possiblities for studies on the effects of different turbulence forcings.

This thesis is centered around the measurement of ultra-high Reynolds numbers, for which the active grid is driven with the aim to generate energy injection scales of different sizes. The relevant grid parameters is the spatial correlation length $\sigma_{S}$ and the temporal correlation length $\sigma_{T}$. $\sigma_{S}$ determines the energy injection scale in the spanwise (transverse) directions, while $\sigma_{T} U$ determines the injection scale in the streamwise (longitudinal) direction at a given mean flow velocity $U$. The volume of a correlation is therefore given by

$$
V_{\text {corr }}=\sigma_{S}^{2} \sigma_{T} U
$$



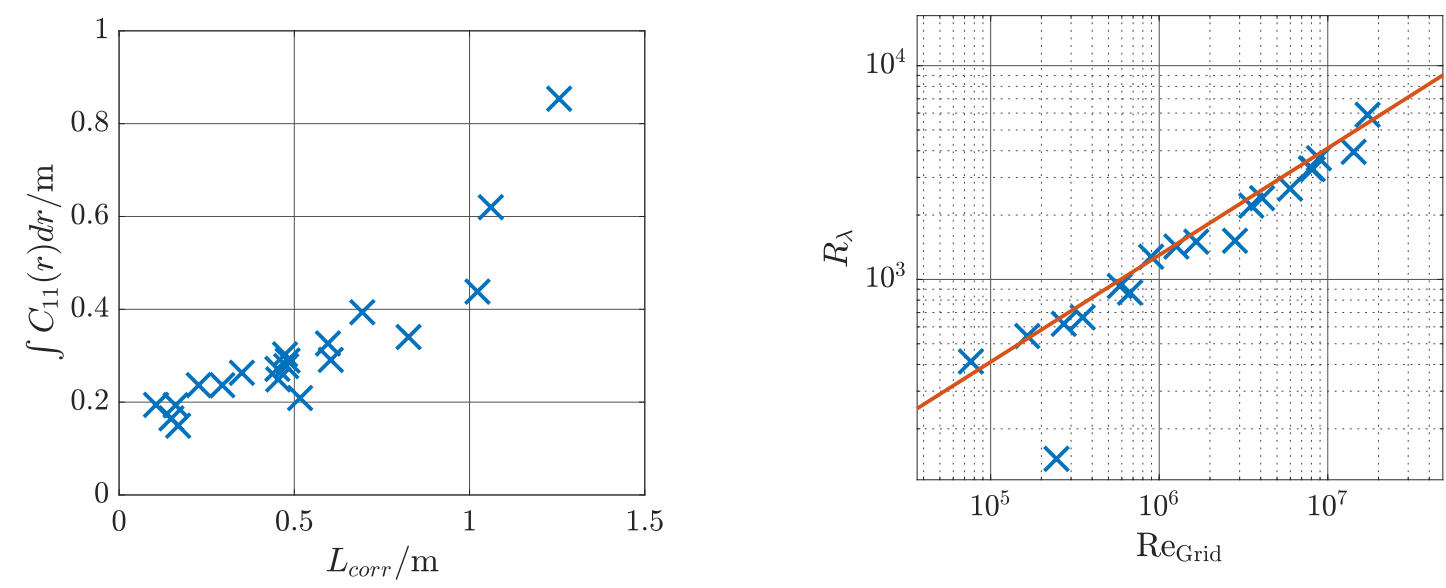

Figure 4.6: Left: The flap correlations of the active grid measured by $L_{c o r r}$ determine the correlation length of the velocity time series even at the end of the measurement section about 9m ( $\sim 80$ flap sizes) downstream of the active grid. Therefore, the energy injection scale is efficiently controlled by the active grid correlations. Right: The a priori grid Reynolds number is a good predictor of the final Taylor scale Reynolds number $R_{\lambda}$. The red line shows the function $0.9845 \sqrt{\operatorname{Re}_{\text {Grid }}}$. The outlier corresponds to a case where the grid was stationary and open.

It can be seen as the average fluid volume that is influenced by one grid correlation. It can be converted into a length scale $L_{\text {corr }}=V_{\text {corr }}^{1 / 3}$. Fig 4.6 shows that the scale at which the active grid correlations occur influences the correlation lengths of the velocity time series in the far-field (>8o flap lengths downstream) of the active grid.

To quantify the relation between the active grid and the creation of large Reynolds numbers we define the Reynolds number based on the active grid by

$$
\operatorname{Re}_{\text {Grid }}=\frac{\sin \left(\Phi_{R M S}\right) U L_{c o r r}}{v}
$$

where $\Phi_{R M S}$ is the RMS of the flap angles with respect to the mean flow velocity, i.e. a measure for the grid blockage. $\operatorname{Re}_{\text {Grid }}$ can be calculated purely from input quantities, i.e. before the actual experiment. As can be seen in Fig. 4.6 the grid Reynolds number is a good predictor for the Taylor-scale Reynolds number $R_{\lambda}$, which is based on quantities that are typically only available after conducting an experiment. Moreover, the simple relation $R_{\lambda} \approx 0.985 \sqrt{\operatorname{Re}_{\text {Grid }}}$ is a good description of the data and resembles the isotropic relation between $R_{\lambda}$ and Re. It can therefore be used to estimate the $R_{\lambda}$ completely from a priori quantities, i.e. active grid setting, facility pressure, and mean flow velocity (c.f. Ref. [108], and Sec. 9). 


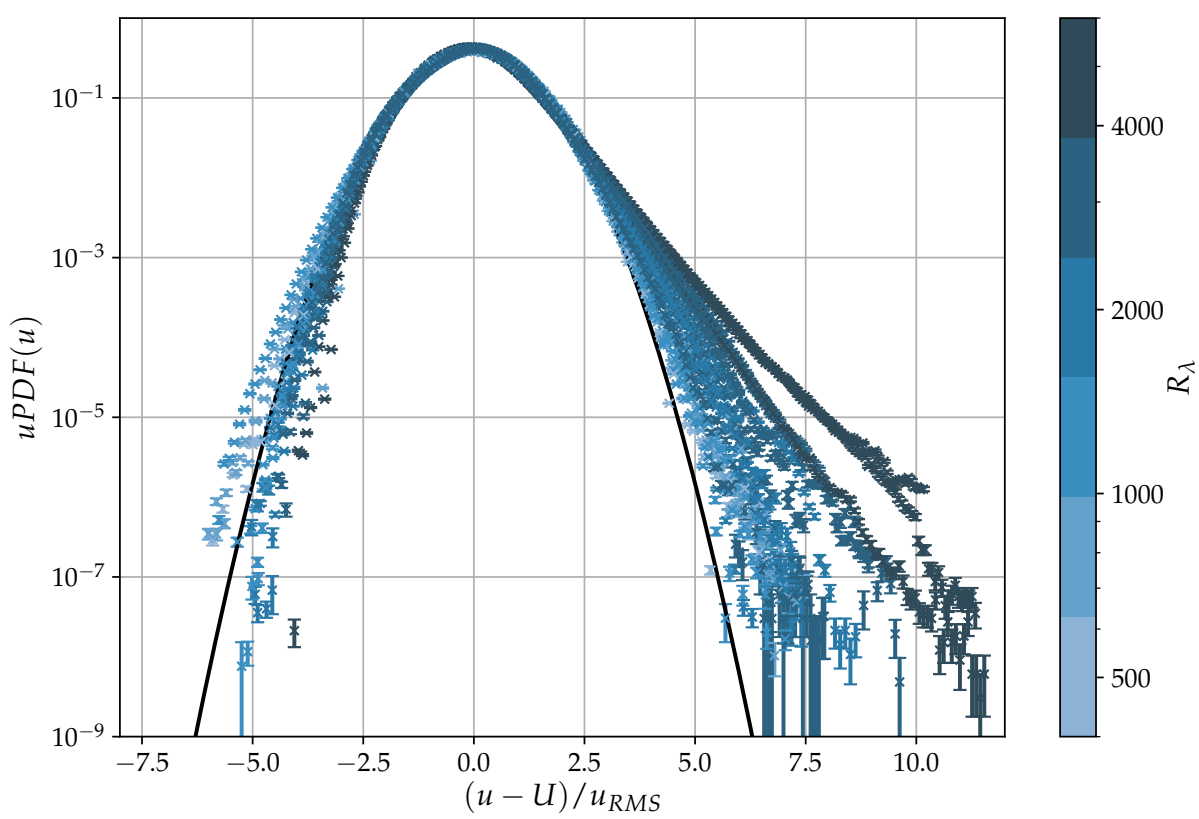

Figure 4.7: Normalised PDFs of the velocity fluctuation color coded by their Taylor-scale Reynolds number $R_{\lambda}$. The black curve is the normal distribution. The measurements show a noticeable deviation from the normal distribution, albeit acceptable in most cases. Three curves have a strong positive skewness. In these cases the active grid was driven in a very anisotropic way to create very large $R_{\lambda}$ (LT3.5LT6, LT5LT10, LT5.2LT15 to reach $R_{\lambda}=4141,5006,5865$, respectively.)

\subsection{Velocity Distributions}

The velocity fluctuations $u$ in a homogeneous turbulent flow are typically assumed to be normally distributed. This means that their flatness $F=\left\langle u^{4}\right\rangle /\left\langle u^{2}\right\rangle^{2}=3$ and their skewness $S=\left\langle u^{3}\right\rangle /\left\langle u^{2}\right\rangle^{3 / 2}=0$. The formal justification for this assumption is the central limit theorem, by which the superposition of independent random variables will be Gaussian distributed [110]. In a turbulent velocity field these random variables are the Fourier coefficients into which the velocity field can be decomposed at any time. However, this justification has to be questioned if the Fourier amplitudes span a wide range of scales. In particular, if the spectrum falls off steeper than $k^{-1}$ as it is typically the case in a turbulent flow, the velocity PDF is expected to be slightly sub-Gaussian [111]. A more comprehensive theoretical treatment [112] yields similar results. These authors find that forced and decaying turbulence are not fundamentally different in their velocity distributions. Still, Maxey [113] applies simple eddy-diffusivity arguments to arrive at the conclusion that in grid turbulence the flux of turbulent kinetic energy in the 
streamwise direction should have a small, positive skewness on the order of the turbulence intensity. Mouri et al. [114] found that the tails of the velocity PDF develop from sub-Gaussian to hyper-Gaussian with increasing distance from their passive grid.

Even though the velocity PDF is strongly influenced by the specific largescale inhomogeneities of real flows, slightly sub-Gaussian statistics are observed in a wide variety of different flows [20, 115, 116]. Metzer \& Klewicki [117] find that skewness and flatness increase with decreasing distance from the turbulent boundary layer. Hearst [118] carried out an extensive study of the wake of a fractal grid and found that skewness and flatness of the velocity distributions deviate from their Gaussian values only in the near-field of the grid (<30 mesh sizes). Similarly, in a strongly anisotropic wind tunnel flow, skewness and flatness settled to (sub-)Gaussian values quickly behind the turbulence generator (an active grid with only three or four horizontal rows) [119]. In summary, the a priori expectation for the velocity distribution in grid turbulence appears to be a Gaussian shape, but deviations from this shape (even strong ones) are not entirely surprising. In the following, the shape of the distributions measured in the VDTT with an active grid shall be described and analysed. The data was sampled with the hot wire system described in 5.3.1 and corresponds to that of Tab. 11.1. The normalised PDFs measured with our constant temperature hot wire setup are shown in Fig. 4.7. With the exception of the case where the active grid was constantly in its most open state (i.e. closely representing a passive grid) the PDFs are skewed towards higher velocities. The majority of them show a skewness $-0.1<S_{u}<0.25$ and a flatness $2.9<F_{u}<3.2$ (see also Fig. 4.8 ). There appears to be a small trend of larger skewness and flatness values towards higher $R_{\lambda}$. Three measurements show very large deviation from a normal distributions. Because the velocity PDF is strongly influenced by the largescale structure of the flow, the dependence of the two shape parameters on the active grid initial conditions and the decay of the flow is investigated in the following.

Fig. 4.8 shows the dependence of the shape parameters on the active grid initial conditions. These measurements were all taken at the downstream end of the measurement section. Note that they are therefore in different positions when normalising by active grid units. The spatial and temporal correlation lengths $\sigma_{S}$ and $\sigma_{T}$ were introduced in Sec. 4.2. The quantity $\sigma_{T} U / \sigma_{S}$ is the dimensionless grid anisotropy that describes the ratio between streamwise (temporal) and transverse (spatial) correlations introduced by the grid. We 

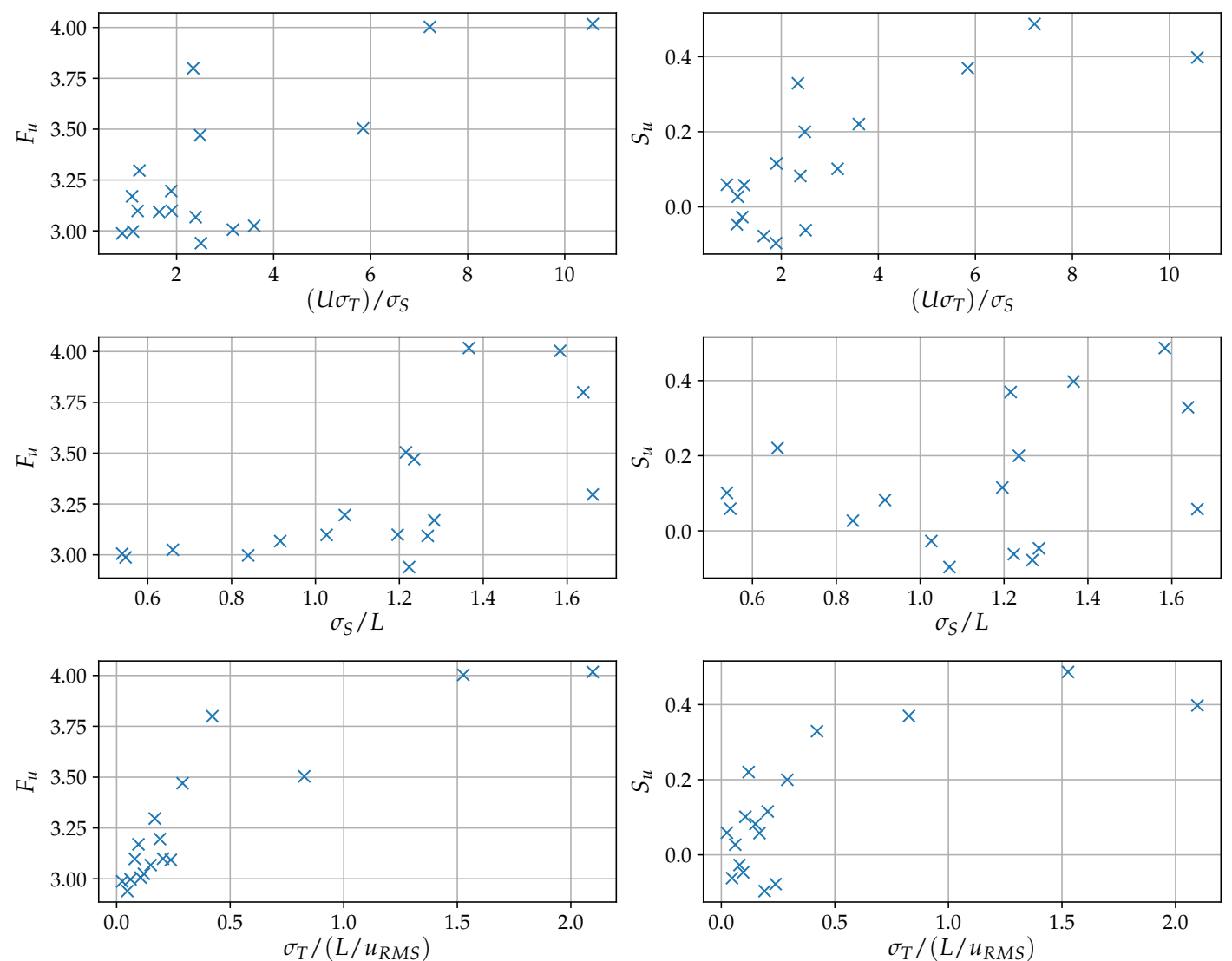

Figure 4.8: Dependence of the PDF shape on the active grid initial conditions. The temporal correlation length $U \sigma_{T}$ appears to be the main contributor to both increased flatness and skewness, whereas the PDF shape is relatively independent of the spatial correlation length $\sigma_{T}$ 

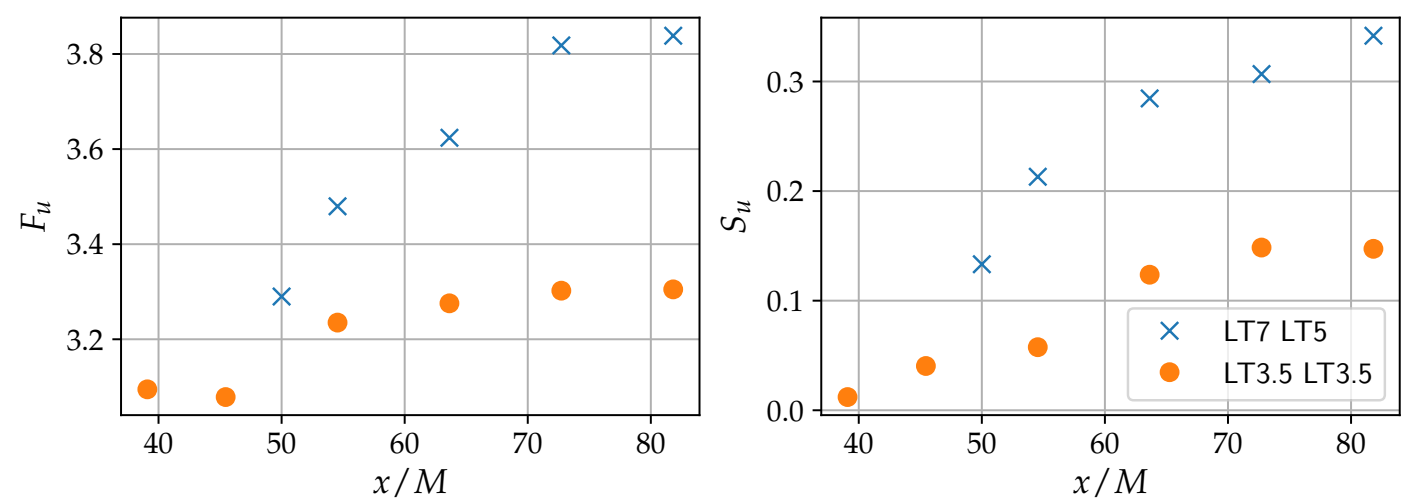

Figure 4.9: Dependence of the PDF shape on the distance from the active grid normalised by the flap size $M=0.11 \mathrm{~m}$ for two different grid protocols $\left(\mathrm{LT}_{7} \mathrm{LT}_{5}, \mathrm{LT}_{3} . \mathrm{LT}_{3} .5\right.$, see Sec. 4.2 for details). $F_{u}$ and $S_{u}$ deviate more and more from their Gaussian values with increasing distance from the grid, but saturate in both cases. Facility pressure: 5.95 bar, mean flow speed $\approx 3.6 \mathrm{~m} / \mathrm{s}$.

can see from Fig. 4.8 that the PDF skewness and (less clear) flatness is relatively independent of $\sigma_{S}$. The PDF shape is much more sensitive to the streamwise correlation length $\sigma_{T} U$. Larger values of this parameter seem to lead to stronger deviations from a Gaussian velocity distribution.

Refs. $[113,112,118,120]$ suggest that the PDF shape depends on the distance from the turbulence generator. Fig. 4.9 therefore shows $S_{u}$ and $F_{u}$ as a function of the distance from the grid for to different grid protocols. As anticipated by Ref. [113], the skewness increases with increasing distance from the grid $x$. The difference between the two grid protocols becomes more and more pronounced with $x$. It is however difficult to compare these data to other studies or draw further conclusions, because the interpretation depends sensitively on the choice of $M$. In our active grid it is not clear, which value to choose. The flap dimensions are certainly a lower bound for the 'grid spacing' $M$, because the their motions are correlated over multiple flaps.

This section concludes by recognising that the PDFs of velocity fluctuations behind our active grid are more complex than in conventional passive grids or even fractal grids [118]. Namely, they are in general flatter than a Gaussian distribution and skewed towards positive fluctuations. This seems to be mainly due to the streamwise correlations introduced by the active grid. If this correlation length $U \sigma_{T}<0.4$, skewness and flatness are restricted to acceptable values. This was the case in most, but not all of our measurements. A velocity distribution close to a Gaussian is 'desirable' for a homogeneous flow, but not strictly necessary. In summary, particular attention should be paid to those cases, where $U \sigma_{T}>0.4$, and this limit should be exceeded only 
if strictly necessary. Furthermore, the influence of the decay on the PDF shape should be investigated in greater detail and the meaning of $M$ in the context of the VDTT active grid should be elucidated.

\subsection{Decay of Turbulent Kinetic Energy}

Even though the turbulence in this experiment is actively stirred throughout a single experiment, the flow has to be regarded as non-stationary. The turbulence in a volume of fluid is excited at one location in the tunnel (the location of the active grid) and then carried with the mean flow without further injection of energy (except wall friction). Therefore, the energy injected at some scale $L$ will be transported to smaller and smaller scales, where it is dissipated by viscosity into heat. Because there is no other mechanism to replace the dissipated energy, the turbulent kinetic energy in the fluid volume is decaying. The way turbulent kinetic energy decays in (grid) turbulence has been investigated in-depth for decades [121, 122, 123, 124, 125, 126, 127, 40]. A very brief summary of the findings could read as follows [40]: The turbulent kinetic energy $u^{2}$ in an unforced fully developed turbulence decays with a power law $t^{n}$ with $-2<n<-0.9$ over time (the exception of exponential decay [124] is noted). The length scales grow also with power laws, unless suppressed by the boundaries (e.g. [128]). During the decay certain quantities remain constant, namely either the Loitsyansky integral

$$
I=-\int \mathbf{r}^{2}\left\langle\mathbf{u} \cdot \mathbf{u}^{\prime}\right\rangle d \mathbf{r}
$$

which implies a constant, but finite global angular momentum of the flow, or the Saffman integral

$$
L=\int\left\langle\mathbf{u} \cdot \mathbf{u}^{\prime}\right\rangle d \mathbf{r}
$$

which is connected to the linear momentum of the flow. In the former case, the small-wavenumber part of the energy spectrum is expected to grow as $E(k) \sim k^{4}$ and $n=-10 / 7$, in the latter case $E(k) \sim k^{2}$ and $n=-6 / 5$ is found. In practice, both laws are observed, with passive grid turbulence usually preferring the latter (e.g. [95]).

The attractiveness of decaying turbulence behind a (passive) grid is that it is known to be very close to the canonical ideal of homogeneous isotropic turbulence [97] (with the obvious exception of the streamwise inhomogeneity due to the presence of a mean flow). The main purpose of this section is to 

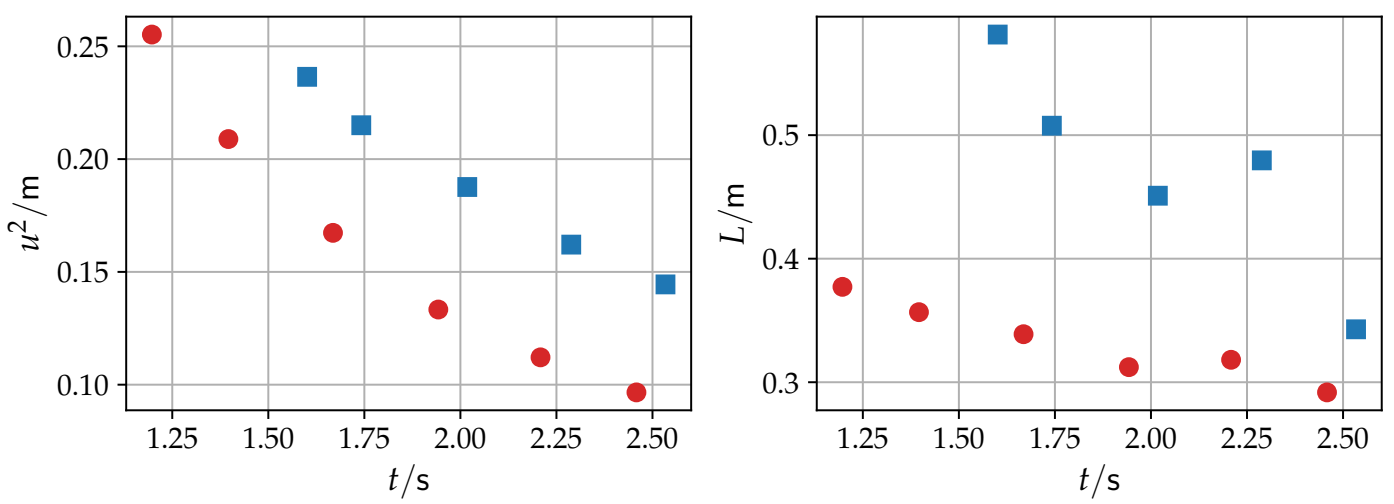

Figure 4.10: Decay of turbulent kinetic energy and development of the energy injection scale $L$ as function of time. $L$ was estimated as $\int C_{11}(r) d r$ in this case. Two different grid protocols are shown (blue squares: $\mathrm{LT}_{7} \mathrm{LT}_{5}$, red circles: $\mathrm{LT}_{3} . \mathrm{LT}_{3} .5$, see Sec. 4.2 for details). The kinetic energy decays as a power law. In contrast to freely decaying turbulence, the size of the energy injection scale does not grow. This is most likely associated to the confinement of the flow in the transverse directions. $t$ is a Galilei transform from the downstream distance from the active grid and the mean flow velocity $U$.

verify that the measurements we take are recorded in the decaying part of the active grid wake in contrast to the near-field buildup. Fig. 4.10 illustrates that the turbulence in the VDTT with an active grid is decaying for both grid protocols tested. As they represent a strongly correlated forcing (blue squares) as well as a more moderate forcing (red circles), we deduce that the active grid in general produces decaying turbulence. As the right panel of Fig. 4.10 shows, the turbulence is not freely decaying, because the largest scales do not grow, but decrease. This is most likely because the growing wall boundary layers confine the flow to a shrinking domain. This effect is less severe when estimating $L=u^{3} / \varepsilon$ (not shown). Since the purpose of this experiment was merely to confirm the decaying nature of the flow, the nearfield was not examined. It is thus not possible to identify the beginning of the decay region $\left(t_{0}\right)$ from this data. Therefore, crucial information to measure the decay exponent $n$ is missing. Sinhuber et al. instead related $u^{2}(t)$ to $L(t)$ to distinguish between the decay theories (4.4) and (4.5), but because $L$ is not growing in the present case, $n$ cannot be extracted this way. In theory, decaying turbulence with $L=$ const. leads to $n=2$ [129].

\subsection{Flow Profiles}

This thesis aims to contribute to the field of homogeneous, and isotropic turbulence. It is therefore of interest to verify that the flow under study is to a reasonable extent homogeneous and isotropic. To measure the isotropy 
of the flow, it is necessary to measure two velocity components. This can be achieved in principle using a pair of crossed hot wires [130], but sensors at the degree of miniaturisation necessary in the VDTT are still in their infancy [131] and their directional calibration in a pressure vessel is a technical challenge. Preliminary data from a Lagrangian particle tracking system (see Ch. 11) suggests a significant presence of anisotropy that depends on the employed active grid forcing. The author therefore had to rely on indirect methods to verify the isotropy of the flow, the knowledge that wind tunnel flows behind a grid are in general close to isotropic [97], and evidence from passive grid measurements inside the VDTT [36]. Bodenschatz et al. [36] have acquired flow profiles along the height of the wind tunnel measurement section (total height $H$ ). They have shown that the mean flow is approximately homogeneous between $0.3 \mathrm{H}$ and $0.6 \mathrm{H}$ with a visible deficit around $0.5 \mathrm{H}$. For the turbulence intensity $u / U$ the homogeneous region is about $0.05 H$ higher with a small local maximum around $0.6 H$. They also find that the flow has an isotropy ratio $v / u \approx 1.1$, as expected for passive grid turbulence[97]. Note that the measurement section was about $12 \mathrm{~cm}$ higher in their case.

In the following a similar set of vertical profiles is presented. The data was acquired in the VDTT filled with 2.92 bar of SF6. The rotation rate of the wind tunnel fan was set at $19.2 \mathrm{~Hz}$ (slightly lower than in Ref. [36] to allow a larger calibration range relative to the measurement speed). Two 450 $\mathrm{m}$ conventional hot wires were mounted on a vertical traverse of $500 \mathrm{~mm}$ travel and driven by a Dantec StreamWare CTA. The traverse was positioned at the downstream end of the measurement section. When the traverse was at the position closest to the floor of the measurement section, one probe was $21.6 \mathrm{~cm}$ above the floor and $9.5 \mathrm{~cm}$ away from the center of the section, while the other was $34.5 \mathrm{~cm}(11.0 \mathrm{~cm})$ above the floor (away from the center). Velocity time series at different heights spaced $1 \mathrm{~cm}$ were recorded for 90 seconds. The measurements were repeated with three different grid protocols (LT1LT1, $\mathrm{LT}_{3.5} \mathrm{LT}_{5}$, and $\mathrm{LT}_{7} \mathrm{LT}_{5}$, see Sec. 4.2 for details), and the mean flap angle was always set at $40^{\circ}$. The original active grid code was adjusted to include the option of decreased solidity at the measurement section boundaries. The flap angles of the four outermost rows could be reduced by a constant factor from their prescribed values. This way, the correlation lengths were kept constant, but the grid solidity was slightly reduced with the aim of decreasing the size of the boundary layers.

Fig. 4.11 shows profiles of the mean velocity acquired from those measurements. The distance from the tunnel floor $y$ has been normalised by the 

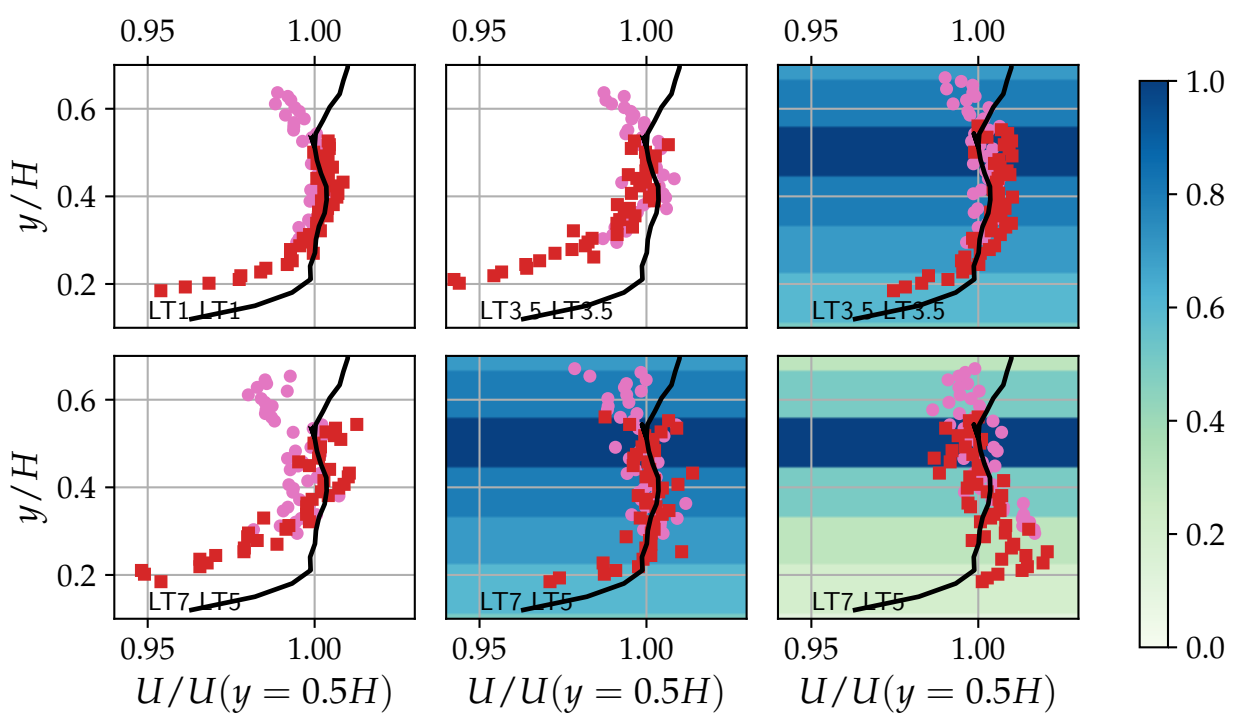

Figure 4.11: Vertical velocity profiles for different grid protocols. Heights are normalised by the measurement section total height $H$, velocities are normalised by the velocity at the center of the measurement section. Vertical profiles with colored background were taken with adjusted active grid solidity: The outer flap angles of the active grid were continuously reduced by a constant factor $\beta$. Lighter color correspond to more open flaps. The black line is the velocity profile measured in the VDTT with a passive grid installed [36]. Different marker colors indicate different probes.

total height of the measurement section $H$. The mean velocity $U$ has been normalised by its value at $y=0.5 H$. This removed some of the existing differences in the measured values of $U$ between the two probes at similar positions. These differences were within the expected calibration errors typical for CTA measurements [132]. Flow profiles, without boundary layer reduction through the active grid (white backgrounds) are approximately homogeneous only between $0.3 \lesssim y / H \lesssim 0.55$. These profiles are most representative of the situations that were investigated in Pt. II (see Appendix B). Active grid protocols $\mathrm{LT}_{3} \cdot 5 \mathrm{LT}_{3} .5$ and $\mathrm{LT}_{7} \mathrm{LT}_{5}$ show more scatter than $\mathrm{LT}_{1} \mathrm{LT}_{1}$, which is attributed to the inferior relative convergence of the statistics due to the larger eddy turnover time and the overall increased turbulence intensity. The flow profile of LT1 $1 \mathrm{LT}_{1}$ does not seem to be perfectly flat at any point. $\mathrm{LT}_{7} \mathrm{LT}_{5}$ appears to show the velocity deficit at the center of the tunnel already observed in the passive grid case [36].

Fig. 4.12 shows the RMS value of the velocity fluctuations normalised by their value at $y / H=0.5$. The regular LT1LT1 and LT3.5LT3.5 profiles show similar boundary layers as deduced from Fig. 4.11. It is notable that the fluctuation intensity in the homogeneous part of the flow (bulk) is less different for large active grid correlation lengths (LT7 $\left.\mathrm{LT}_{5}, \mathrm{LT}_{3} .5 \mathrm{LT}_{3} \cdot 5\right)$ than 


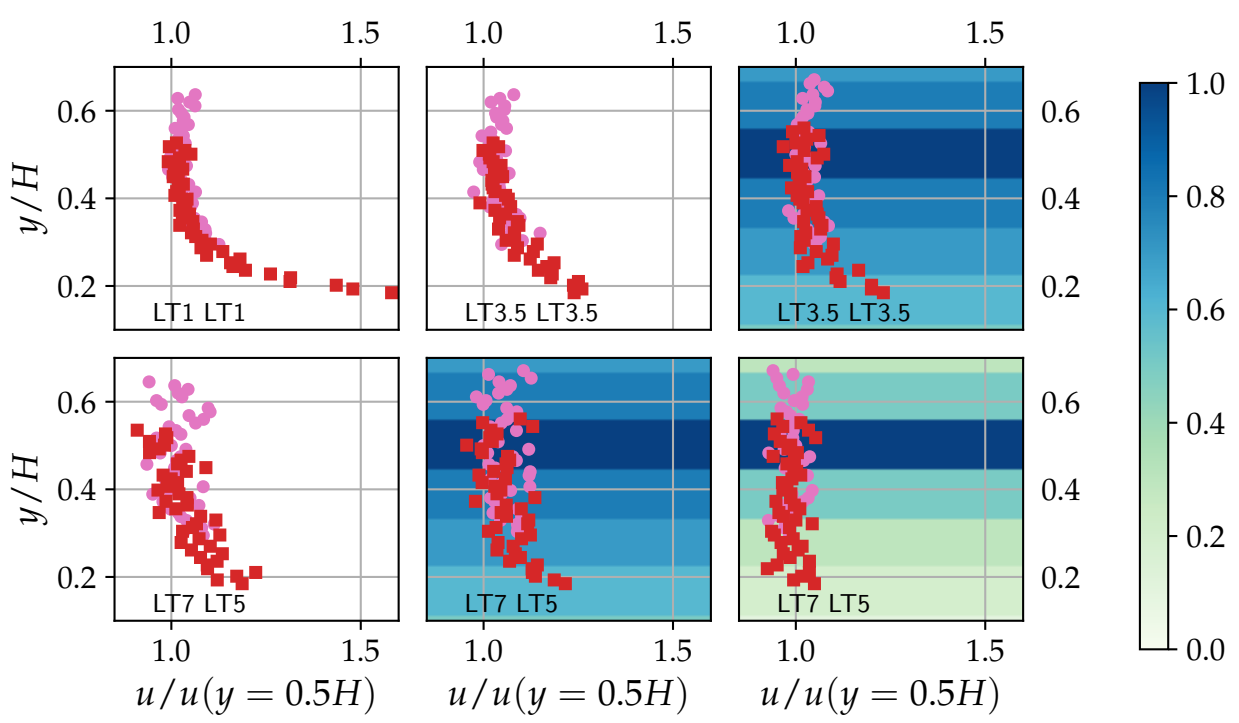

Figure 4.12: Vertical profiles of the velocity fluctuations for different grid protocols. Heights are normalised by the measurement section total height $H$, velocities are normalised by the velocity at the center of the measurement section. Vertical profiles with colored background were taken with adjusted active grid solidity: The outer flap angles of the active grid were continuously reduced by a constant factor. Lighter colors correspond to more open flaps.

for smaller correlation lengths (LTILTI). A possible explanation is that the larger Reynolds number of these flows increase the mixing between the bulk and the boundary layer and therefore cause the their fluctuation intensity to become more similar.

Fig. 4.13 shows the values of $\partial U / \partial y$ for different heights. This quantity is a measure for the mean shear of the flow, which is an indication for the presence of anisotropy [105]. To obtain the gradient, the data from Fig. 4.11 has been smoothed by a moving average of kernel size 9 and the second-order numerical derivative of the smoothed data was calculated.

For the cases in which the active grid solidity close to the tunnel boundary was not reduced (white background), only a relatively small region is approximately free of mean shear $(0.4<y / H<0.5)$. This is largely independent of the grid protocol used. The data for grid protocol $\mathrm{LT}_{7} \mathrm{LT}_{5}$ would justify a slightly larger shear-free region due to the large scatter.

In addition to the investigation of the regular grid protocols used to obtain the statistics presented in Part II, the possibility of minimising the mean shear present in the system by adjusting the active grid flap angles was explored. Mean shear is produced by the friction between the moving fluid and the stationary tunnel walls slowing the fluid down. This could be counteracted by providing the regions of the flow close to the tunnel boundaries with 


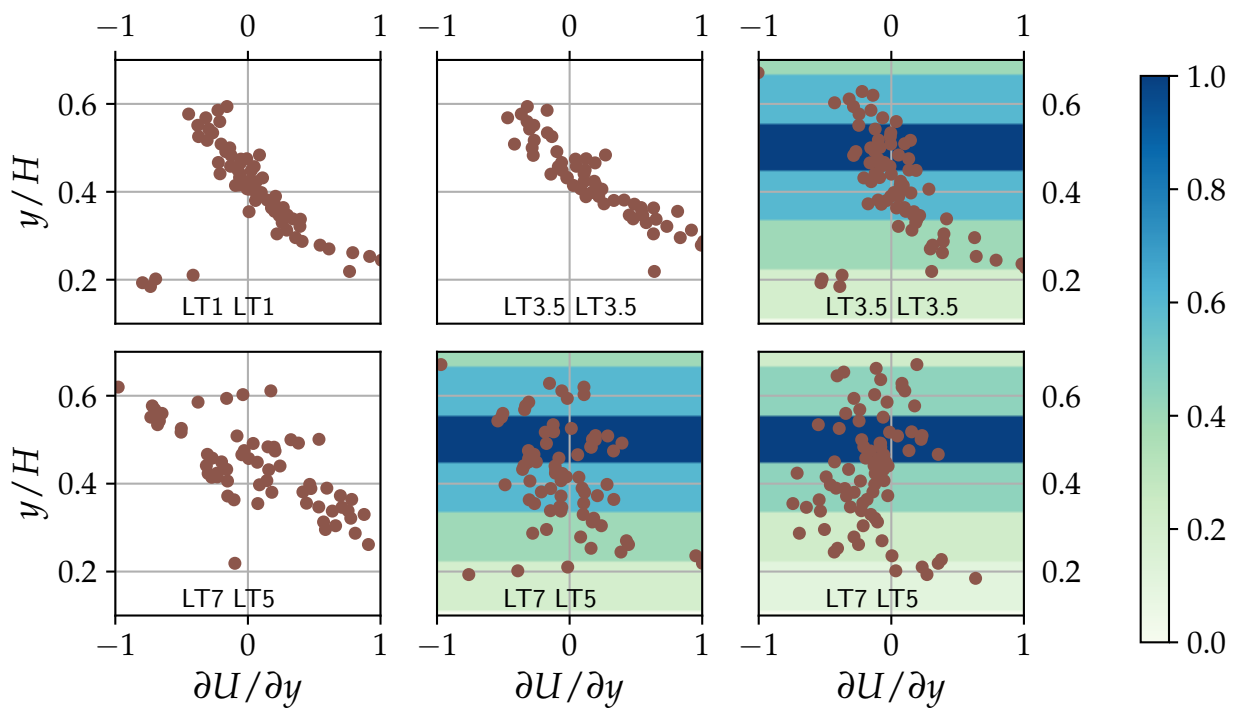

Figure 4.13: Profiles of mean shear along the tunnel height for different grid protocols. The data was obtained by applying a moving average smoothing to the data in 4.11. Vertical profiles with coloured background were taken with adjusted active grid solidity. The outer flap angles of the active grid continuously reduced by a constant factor. Lighter colours correspond to more open flaps.

additional kinetic energy relative to the bulk flow.

To this end, the active grid algorithm introduced in [133] and described in 4.2 was adjusted as follows: Each of the four outermost rings of flaps has a constant factor assigned to it. The algorithm produced the sets of grid angles in the regular way, but before these sets were sent to the servo motors to move the active grid flaps, the angles of the outer rings of angles were multiplied by the constant factor $\beta$ set in the program. For example, if the regular setting of the RMS of grid angles was set to $50^{\circ}$, and the outermost ring had a boundary correction factor of 0.1 set to them, the RMS of the outermost ring would be calculated as $5^{\circ}$. Assuming the next ring of flaps closer to the center had $\beta=0.3$ assigned to it, the RMS angle of this ring would be $15^{\circ}$. Since smaller active grid angles cause smaller flow obstruction, it is expected that this technique causes higher mean flow velocities on the edges of the tunnel and thus less mean shear.

Figs. 4.11, 4.12, and 4.13 confirm this expectation to a good extent. To obtain the upper right and lower middle plots of this figures, the outer rings were set to $\beta_{1}=0.5, \beta_{2}=0.6, \beta_{3}=0.7, \beta_{4}=0.8$, where index 1 corresponds to the outermost ring of flaps. To obtain the lower right plot in these figures, $\beta_{1-4}$ was set to $0.1,0.2,0.3,0.5$, respectively. Figs. 4.11, 4.12, and 4.13 clearly show that this procedure reduces the size of the boundary layers and brings 
the flow profile closer to that of the passive grid. The homogeneous region becomes larger and the mean shear is reduced albeit large scatter. However, when applying a strong boundary layer correction as in the lower right plots, a small constant mean shear appears instead of a homogeneous region as shown in Fig. 4.11. The profile of the velocity fluctuations is constant across the entire height investigated, but Fig. 4.13 shows clearly the existence of a relatively constant nonzero value of $\partial U / \partial y$. The reason for this behaviour is not clear at this moment and demands further investigation.

In summary, the flow shows only a small homogenous region when using the active grid without correction of the boundary layer. This was the case for all hot wire measurements. These results are therefore likely influenced by large-scale inhomogeneities, and large-scale anisotropies. The degree of inhomogeneity and anisotropy is however small compared to those considered by e.g. Shen \& Warhaft [104]. These authors consider a purely shear-driven flow, where the mean velocity changes by a factor of 3 over a similar range of $y$ as considered here. They find that at second order, the postulate of local isotropy is valid even in strongly sheared turbulence, but departures begin already at third order. Chang et al. [134] similarly find that anisotropy in the absence of shear influences the inertial range statistics at levels below their experimental uncertainty.

In the future, the use of the boundary layer correction is strongly recommended since it significantly increases the homogeneity of the flow and most likely reduces the level of anisotropy. The new feature is also a prime example of the flexibility of active grids. The boundary layer correction can in principle also be used to improve active grids after the example of Makita [99], where only rows and columns can be controlled individually.

\subsection{Summary}

This thesis presents data from the wind tunnel that achieves the largest Taylor-scale Reynolds number in the world (at the point of writing) under well-controlled conditions. This is possible due to the unique combination of a low-viscosity pressurised gas (SF6) and a very flexible active grid. The turbulence created in such a way is decaying, its velocity PDFs are slightly skewed and super-Gaussian, and the vertical velocity profiles have a small, but sufficient homogeneous region, which can be extended by adjusting the active grid. To obtain extreme $R_{\lambda}$, some of the "cleanliness" of passive grid turbulence had to be sacrificed. It is therefore of great importance to verify 
that the conclusions drawn from the active grid data are robust against different active grid configurations and comparisons to the (isotropic) passive grid data presented most throughly in Ref. [93] are to be carried out whenever possible given the lower $R_{\lambda}$ of that data. Thus, when care is being taken, the data from the VDTT with the active grid can contribute to the knowledge about (closely) homogeneous and isotropic turbulence. 


\section{Bibliography}

[1] R. Ecke. The Theory of Turbulence. Ed. by E. A. Spiegel. Vol. 81o. Lecture Notes in Physics. Dordrecht: Springer Netherlands, 2011. DoI: 10. 1007/978-94-007-0117-5.

[2] A. Tsinober. An Informal Introduction to Turbulence. Vol. 63. 2004, pp. 277311. DOI: $10.1007 / 0-306-48384-\mathrm{x}$.

[3] O. Reynolds. "An experimental investigation of the circumstances which determine whether the motion of water shall be direct or sinuous, and of the law of resistance in parallel channels". In: Philos. Trans. R. Soc. London 174 (1883), pp. 935-982. DOI: 10.1098/rst1.1883.0029.

[4] K. Avila et al. "The Onset of Turbulence in Pipe Flow". In: Science (80-. ). 333.6039 (July 2011), pp. 192-196. DOI: 10.1126/science.1203223.

[5] E. Bodenschatz et al. "Can We Understand Clouds Without Turbulence?" In: Science (80-. ). 327.5968 (Feb. 2010), pp. 970-971. DOI: 10 . 1126/science. 1185138.

[6] S. Thorpe. "Recent Developments In The Study Of Ocean Turbulence". In: Annu. Rev. Earth Planet. Sci. 32.1 (May 2004), pp. 91-109. Dor: 10 . 1146/annurev . earth. 32.071603.152635.

[7] F. Veron. "Ocean Spray". In: Annu. Rev. Fluid Mech. 47.1 (Jan. 2015), pp. 507-538. DOI: 10.1146/annurev-fluid-010814-014651.

[8] R. E. Breier et al. "Emergence of phytoplankton patchiness at small scales in mild turbulence". In: Proc. Natl. Acad. Sci. U. S. A. 115.48 (2018), pp. 12112-12117. DOI: 10.1073/pnas.1808711115.

[9] H. Yamazaki and T. R. Osborn. "Review of Oceanic Turbulence: Implications for Biodynamics". In: Towar. a Theory Biol. Interact. World Ocean. Yamazaki1988: Springer Netherlands, 1988, pp. 215-234. DOI: 10.1007/978-94-009-3023-0_12.

[10] J. Reneaux. "Overview on drag reduction technologies for civil transport aircraft". In: ECCOMAS 2004 - Eur. Congr. Comput. Methods Appl. Sci. Eng. 2004.

[11] D. Xu et al. "Nonlinear hydrodynamic instability and turbulence in pulsatile flow". In: Proc. Natl. Acad. Sci. 117.21 (May 2020), pp. 1123311239. DOI: $10.1073 /$ pnas. 1913716117. 
[12] P. Milan, M. Wächter, and J. Peinke. "Turbulent Character of Wind Energy". In: Phys. Rev. Lett. 110.13 (Mar. 2013), p. 138701. DoI: 10 . 1103/PhysRevLett.110.138701.

[13] M. A. Miller et al. "Horizontal axis wind turbine testing at high Reynolds numbers". In: Phys. Rev. Fluids 4.11 (Nov. 2019), p. 110504. DOI: 10 . 1103/PhysRevFluids.4.110504.

[14] J. Peinke et al. "Turbulence, a challenging problem for wind energy". In: Phys. A Stat. Mech. its Appl. 2004. DOI: 10.1016/j . physa. 2004.02 . 040.

[15] T. Mücke, D. Kleinhans, and J. Peinke. "Atmospheric turbulence and its influence on the alternating loads on wind turbines". In: Wind Energy (2011). DOI: 10.1002/we.422.

[16] A. H. Boozer. "Physics of magnetically confined plasmas". In: Rev. Mod. Phys. 76.4 (Jan. 2005), pp. 1071-1141. Dor: 10.1103/RevModPhys . 76.1071.

[17] T. Klinger et al. "Overview of first Wendelstein 7 - $X$ high-performance operation". In: Nucl. Fusion 59.11 (Nov. 2019), p. 112004. DOI: 10.1088/ $1741-4326 / \mathrm{ab} 03 \mathrm{a} 7$.

[18] G. I. Taylor. "Statistical Theory of Turbulence". In: Proc. R. Soc. A Math. Phys. Eng. Sci. 151.873 (Sept. 1935), pp. 421-444. DOI: 10.1098/rspa. 1935.0158.

[19] K. R. Sreenivasan, A. Prabhu, and R. Narasimha. "Zero-crossings in turbulent signals". In: J. Fluid Mech. 137.-1 (Dec. 1983), p. 251. DOI: 10.1017/S0022112083002396.

[20] K. R. Sreenivasan and B. Dhruva. "Is There Scaling in High-ReynoldsNumber Turbulence?" English. In: Prog. Theor. Phys. Suppl. 130 (1998), pp. 103-120. DOI: 10.1143/PTPS.130.103.

[21] M. Sinhuber. "On the Scales of Turbulent Motion at High Reynolds Numbers". PhD thesis. Georg-August-Universität Göttingen, 2015.

[22] H. Kahalerras et al. "Intermittency and Reynolds number". In: Phys. Fluids 10.4 (Oct. 1998), p. 910. DOI: 10.1063/1.869613.

[23] M. Bourgoin et al. "Investigation of the small-scale statistics of turbulence in the Modane SIMA wind tunnel". In: CEAS Aeronaut. J. 9.2 (June 2018), pp. 269-281. DOI: 10.1007/s13272-017-0254-3. 
[24] B. Rousset et al. "Superfluid high REynolds von Kármán experiment". In: Rev. Sci. Instrum. 85.10 (Oct. 2014), p. 103908. DOI: 10.1063/1. 4897542.

[25] S. Pietropinto et al. "Superconducting instrumentation for high Reynolds turbulence experiments with low temperature gaseous helium". In: Phys. C Supercond. 386 (Apr. 2003), pp. 512-516. Dor: 10.1016/S0921 4534 (02) 02115-9.

[26] G. Gulitski et al. "Velocity and temperature derivatives in high-Reynoldsnumber turbulent flows in the atmospheric surface layer. Part 2. Accelerations and related matters". In: J. Fluid Mech. 589 (2007), pp. 83-102. DOI: $10.1017 / \mathrm{S} 0022112007007501$.

[27] S. Risius et al. "Schneefernerhaus as a mountain research station for clouds and turbulence". In: Atmos. Meas. Tech. (2015). DoI: 10.5194/ amt-8-3209-2015.

[28] H. Siebert et al. "High-resolution measurement of cloud microphysics and turbulence at a mountaintop station". In: Atmos. Meas. Tech. 8.8 (Aug. 2015), pp. 3219-3228. DOI: 10.5194/amt-8-3219-2015.

[29] G. Bagheri et al. "Cloudkite: an airborne platform for resolving clouds". In: Geophys. Res. Abstr. EGU, 2018.

[30] C. Canter and S. C. Bailey. "Spatially Sampled Turbulence Measurements Acquired Using Unmanned Aerial Vehicles". In: 71st Annu. Meet. APS Div. Fluid Dyn. Atlanta, Georgia, 2018.

[31] P. K. Yeung, K. R. Sreenivasan, and S. B. Pope. “Effects of finite spatial and temporal resolution in direct numerical simulations of incompressible isotropic turbulence". In: Phys. Rev. Fluids 3.6 (2018), pp. 115. DOI: 10.1103/PhysRevFluids.3.064603.

[32] T. Ishihara. "Energy spectrum in high-resolution direct numerical". In: 082403 (2016), pp. 1-9. DOI: 10.1103/PhysRevFluids.1.082403.

[33] S. A. Orszag and G. S. Patterson. "Numerical simulation of threedimensional homogeneous isotropic turbulence". In: Phys. Rev. Lett. (1972). DOI: 10.1103/PhysRevLett.28.76.

[34] M. V. Zagarola and A. J. Smits. "Scaling of the mean velocity profile for turbulent pipe flow". In: Phys. Rev. Lett. (1997). Dor: 10.1103/ PhysRevLett.78.239. 
[35] J. Salort et al. "A local sensor for joint temperature and velocity measurements in turbulent flows". In: Rev. Sci. Instrum. 89.1 (Jan. 2018), p. 015005. DOI: 10.1063/1.4989430.

[36] E. Bodenschatz et al. "Variable Density Turbulence Tunnel Facility". In: Rev. Sci. Instrum. 85.9 (2014), pp. 1-45. DOI: 10.1063/1.4896138.

[37] L. Mydlarski and Z. Warhaft. "On the onset of high-Reynolds-number grid-generated wind tunnel turbulence". In: J. Fluid Mech. 320.-1 (Aug. 1996), p. 331. DOI: 10.1017/S0022112096007562.

[38] L. Neuhaus, J. Peinke, and M. Hölling. "High Reynolds number turbulence generation by active grid and wind tunnel control". In: Eur. Turbul. Conf. 17. 2019.

[39] ONERA. SiMA L arge T ransonic A tmospheric $W$ ind $T$ unnel. Tech. rep. 2006.

[40] P. Davidson. Turbulence - An Introduction For Scientists and Engineers. Oxford University Press, 2015.

[41] A. S. Monin and A. M. Yaglom. Statistical Fluid Mechanics Vol. II. Dover, 1975 .

[42] S. B. Pope. "Turbulent Flows". In: Cambridge Univ. Press. Vol. 1. 2000. Chap. 6 - Scalin, pp. 232-233. DOI: 10.1088/1468-5248/1/1/702.

[43] M. Farge. "Wavelet Transforms And Their Applications To Turbulence”. In: Annu. Rev. Fluid Mech. (1992). Dor: 10.1146/annurev .fluid.24.1. 395.

[44] J. Boschung et al. "Finite Reynolds number corrections of the 4/5 law for decaying turbulence". In: Phys. Rev. Fluids 1.6 (Oct. 2016), p. 064403. DOI: 10.1103/PhysRevFluids.1.064403.

[45] R. J. HILL. "Equations relating structure functions of all orders". In: J. Fluid Mech. 434 (May 2001), pp. 379-388. DOI: 10.1017/S0022112001003949.

[46] M. Millionshchikov. "On the theory of homogeneous isotropic turbulence". In: Dokl. Akad. Nauk SSSR 32.9 (1941), pp. 611-614.

[47] S. A. Orszag. "Analytical theories of turbulence". In: J. Fluid Mech. 41.2 (Apr. 1970), pp. 363-386. DOI: 10.1017/S0022112070000642.

[48] P. Sagaut and C. Cambon. Homogeneous turbulence dynamics. 2018. DoI: 10.1007/978-3-319-73162-9. 
[49] A. N. Kolmogorov. "Dissipation of energy in the locally isotropic turbulence". In: Dokl. Akad. Nauk SSSR 32.1 (1941). DoI: 10.1098/rspa. 1991.0076.

[5o] J. Monaghan and J. Kajtar. "Leonardo da Vinci's turbulent tank in two dimensions". In: Eur. J. Mech. - B/Fluids 44 (Mar. 2014), pp. 1-9. DoI: 10.1016/j.euromechflu.2013.09.005.

[51] L. F. Richardson. "Weather Prediction by Numerical Process". en. In: Cambridge Univ. Press. Cambridge mathematical library (1922), p. 236. DOI: $10.2136 /$ sssaj1966.03615995003000010006x.

[52] C. F. v. Weizsäcker. "Das Spektrum der Turbulenz bei großen Reynoldsschen Zahlen". In: Zeitschrift für Phys. 124.7-12 (July 1948), pp. 614-627. DOI: $10.1007 / \mathrm{BF} 01668898$.

[53] A. Yaglom. "On the local structure of a temperature field in a turbulent flow". In: Dokl. Akad. Nauk. SSSR (1949).

[54] J. Argyris et al. Die Erforschung des Chaos. 2nd. Berlin, Heidelberg: Springer Berlin Heidelberg, 2010. DOI: 10.978.3540/710714.

[55] B. Mandelbrot. The Fractal Geometry of Nature. 1982.

[56] J. Jimenez. “Turbulence and vortex dynamics". In: Notes Polytecnique course Turbul. (2004).

[57] A. Kolmogorov. "The Local Structure of Turbulence in Incompressible Viscous Fluid for Very Large Reynolds' Numbers". In: Dokl. Akad. Nauk SSSR 30 (1941), pp. 301-305.

[58] D. Lohse and A. Muller-Groeling. "Bottleneck effects in turbulence: Scaling phenomena in r versus p space". In: Phys. Rev. Lett. 74.10 (1995), pp. 1747-1750. DOI: 10.1103/PhysRevLett.74.1747.

[59] B. R. Pearson, P.- A. Krogstad, and W. van de Water. "Measurements of the turbulent energy dissipation rate". In: Phys. Fluids 14.3 (Mar. 2002), pp. 1288-1290. DOI: 10.1063/1.1445422.

[6o] P. Burattini, P. Lavoie, and R. A. Antonia. "On the normalized turbulent energy dissipation rate". In: Phys. Fluids 17.9 (2005), pp. 1-4. DoI: 10 . 1063/1.2055529.

[61] S. Lück et al. "The Markov-Einstein coherence length—a new meaning for the Taylor length in turbulence". In: Phys. Lett. A 359.5 (Dec. 2006), pp. 335-338. DOI: 10.1016/j . physleta.2006.06.053. 
[62] E. Bodenschatz and M. Eckert. "Prandtl and the Göttingen school". In: A Voyag. Through Turbul. Ed. by P. A. Davidson et al. Cambridge: Cambridge University Press, 2011, pp. 40-100. DOI: 10.1017/CB09781139018241. 003.

[63] L. Onsager. "Minutes of the Meeting of the Metropolitan Section held at Columbia University, New York, November 9 and 10, 1945". In: Phys. Rev. 68.11-12 (Dec. 1945), pp. 281-288. DoI: 10.1103/PhysRev. 68.281.

[64] W. Heisenberg. "Zur statistischen Theorie der Turbulenz". In: Zeitschrift für Phys. 124.7-12 (July 1948), pp. 628-657. DoI: 10.1007/BF01668899.

[65] L. Landau and E. Lifshitz. Mechanics of continuus media. 1st Russia. Moscow: Gostekhizdat, 1944.

[66] A. N. Kolmogorov. "A refinement of previous hypotheses concerning the local structure of turbulence in a viscous incompressible fluid at high Reynolds number". In: J. Fluid Mech. 13.1 (May 1962), pp. 82-85. DOI: $10.1017 /$ S0022112062000518.

[67] L. C. Andrews et al. "A statistical theory for the distribution of energy dissipation in intermittent turbulence". In: Phys. Fluids A Fluid Dyn 1.6 (June 1989), pp. 999-1006. DOI: 10.1063/1.857412.

[68] B. Dubrulle. "Intermittency in fully developed turbulence: Log-poisson statistics and generalized scale covariance". In: Phys. Rev. Lett. (1994). DOI: 10.1103/PhysRevLett.73.959.

[69] Z.-S. She and E. Leveque. "Universal scaling laws in fully developed turbulence". In: Phys. Rev. Lett. 72.3 (1994), pp. 336-339. DOI: 10.1103/ PhysRevLett.72.336.

[70] N. Cao, S. Chen, and Z.-S. She. "Scalings and Relative Scalings in the Navier-Stokes Turbulence". In: Phys. Rev. Lett. 76.20 (May 1996), pp. 3711-3714. DOI: 10.1103/PhysRevLett.76.3711.

[71] R. Benzi et al. "Extended self-similarity in turbulent flows". In: Phys. Rev. E 48.1 (1993), pp. 29-32. DOI: 10.1103/PhysRevE.48.R29.

[72] R. Benzi et al. "Multifractal nature of fully developed turbulence and chaotic systems." In: (1984).

[73] U. Frisch, P.-L. Sulem, and M. Nelkin. "A simple dynamical model of intermittent fully developed turbulence". In: J. Fluid Mech. 87.04 (Aug. 1978), p. 719. DOI: 10.1017/S0022112078001846. 
[74] U. Frisch. Turbulence. Cambridge University Press, Nov. 1995. DOI: 10. 1017/CB09781139170666.

[75] K. P. Iyer, K. R. Sreenivasan, and P. K. Yeung. "Circulation in High Reynolds Number Isotropic Turbulence is a Bifractal". In: Phys. Rev. X (2019). DOI: 10.1103/PhysRevX.9.041006.

[76] C. Meneveau and K. R. Sreenivasan. "Simple multifractal cascade model for fully developed turbulence". In: Phys. Rev. Lett. 59.13 (Sept. 1987), pp. 1424-1427. DOI: 10.1103/PhysRevLett.59.1424.

[77] C. Meneveau and K. R. Sreenivasan. "The multifractal nature of turbulent energy dissipation". In: J. Fluid Mech. 224.-1 (Mar. 1991), p. 429. DOI: $10.1017 / \mathrm{S} 0022112091001830$.

[78] U. Frisch and M. Vergassola. "A Prediction of the Multifractal Model: the Intermediate Dissipation Range". In: Europhys. Lett. 14.5 (Mar. 1991), pp. 439-444. DOI: 10.1209/0295-5075/14/5/009.

[79] A. Bennett. Lagrangian Fluid Dynamics. 2006. DoI: 10.1017/cbo9780511734939.

[8o] S. B. Pope. "Lagrangian PDF Methods for Turbulent Flows". In: Annu. Rev. Fluid Mech. 26.1 (Jan. 1994), pp. 23-63. DOI: 10.1146/annurev.f1. 26.010194 .000323$.

[81] B. Sawford. “T URBULENT R ELATIVE D ISPERSION”. In: Annu. Rev. Fluid Mech. 33.1 (Jan. 2001), pp. 289-317. DoI: 10.1146/annurev.fluid. 33.1.289.

[82] N. Mordant et al. "Measurement of lagrangian velocity in fully developed turbulence". In: Phys. Rev. Lett. (2001). DoI: 10.1103/PhysRevLett. 87.214501.

[83] G. A. Voth, K. Satyanarayan, and E. Bodenschatz. "Lagrangian acceleration measurements at large Reynolds numbers". In: Phys. Fluids 10.9 (Oct. 1998), p. 2268. DOI: 10.1063/1.869748.

[84] S. R. Hanna. "Lagrangian and Eulerian time-scale relations in the daytime boundary layer ( Boulder, Colorado)." In: J. Appl. Meteorol. (1981). DOI: $10.1175 / 1520-0450$ (1981)020<0242:LAETSR>2 . 0 . CO 2.

[85] F. Toschi and E. Bodenschatz. "Lagrangian Properties of Particles in Turbulence". In: Annu. Rev. Fluid Mech. 41.1 (Jan. 2009), pp. 375-404. DOI: 10.1146/annurev.fluid.010908.165210. 
[86] S. Ott and J. Mann. "An experimental investigation of the relative diffusion of particle pairs in three-dimensional turbulent flow". In: J. Fluid Mech. (2000). DOI: 10.1017/S0022112000001658.

[87] G. Falkovich, K. Gawedzki, and M. Vergassola. "Particles and fields in fluid turbulence". In: Rev. Mod. Phys. 73.4 (Nov. 2001), pp. 913-975. DOI: 10.1103/RevModPhys .73.913.

[88] T. D. Drivas. "Turbulent Cascade Direction and Lagrangian Time-Asymmetry". In: J. Nonlinear Sci. (2019). DOI: 10.1007/s00332-018-9476-8.

[89] J. Jucha et al. "Time-reversal-symmetry breaking in turbulence". In: Phys. Rev. Lett. (2014). DoI: 10.1103/PhysRevLett.113.054501.

[9o] G. A. VOTH et al. "Measurement of particle accelerations in fully developed turbulence". In: J. Fluid Mech. 469 (Oct. 2002), pp. 121-16o. DoI: 10.1017/S0022112002001842.

[91] A. La Porta et al. "Fluid particle accelerations in fully developed turbulence". In: Nature 409.6823 (Feb. 2001), pp. 1017-1019. DOI: 10.1038/ 35059027.

[92] D. Buaria. "Lagrangian Investigations of Turbulent Dispersion and Mixing Using Petascale Computing". PhD thesis. Georgia Institute of Technology, 2016.

[93] M. Sinhuber. “On the Scales of Turbulent Motion at High Reynolds Numbers". PhD thesis. 2015.

[94] M. Sinhuber, G. P. Bewley, and E. Bodenschatz. "Dissipative Effects on Inertial-Range Statistics at High Reynolds numbers". In: Phys. Rev. Lett. 119.1962 (Sept. 2016), p. 134502. DOI: 10.1103/PhysRevLett.119. 134502.

[95] M. Sinhuber and M. Sinhuber. "On the Scales of Turbulent Motion at High Reynolds Numbers". PhD thesis. Georg-August-Universität Göttingen, 2015.

[96] G. Comte-Bellot and S. Corrsin. "Simple Eulerian time correlation of full-and narrow-band velocity signals in grid-generated, 'isotropic' turbulence". In: J. Fluid Mech. (1971). DOI: 10.1017/S0022112071001599.

[97] G. Comte-Bellot and S. Corrsin. "The use of a contraction to improve the isotropy of grid-generated turbulence". In: J. Fluid Mech. 25.4 (1966), pp. 657-682. DOI: 10.1017/S0022112066000338. 
[98] G. I. Taylor. "The Spectrum of Turbulence". In: Proc. R. Soc. A Math. Phys. Eng. Sci. 164.919 (Feb. 1938), pp. 476-490. DOI: 10.1098/rspa . 1938.0032.

[99] H. Makita and K. Sassa. "Active Turbulence Generation in a Laboratory Wind Tunnel". In: Adv. Turbul. 3. Berlin, Heidelberg: Springer Berlin Heidelberg, 1991, pp. 497-505. DOI: 10.1007/978-3-642-843990_54.

[100] L. Mydlarski. "A turbulent quarter century of active grids: From Makita (1991) to the present". In: Fluid Dyn. Res. 49.6 (2017). DOI: 10.1088 / 1873-7005/aa7786.

[101] L. Mydlarski and Z. Warhaft. "On the onset of high-Reynolds-number grid-generated wind tunnel turbulence". In: J. Fluid Mech. 320.-1 (1996), p. 331. DOI: 10.1017/S0022112096007562.

[102] H. E. Cekli and W. van de Water. "Tailoring turbulence with an active grid". In: Exp. Fluids 49.2 (Jan. 2010), pp. 409-416. DoI: 10.1007 / s00348-009-0812-5.

[103] P. Knebel, A. Kittel, and J. Peinke. "Atmospheric wind field conditions generated by active grids". In: Exp. Fluids 51.2 (Aug. 2011), pp. 471481. DOI: $10.1007 / \mathrm{s} 00348-011-1056-8$.

[104] X. Shen and Z. Warhaft. "Longitudinal and transverse structure functions in sheared and unsheared wind-tunnel turbulence". In: Phys. Fluids 14.1 (2002), pp. 370-381. DOI: 10.1063/1.1421059.

[105] X. Shen and Z. Warhaft. "The anisotropy of the small scale structure in high Reynolds number $(\mathrm{R}(\lambda) \sim 1000)$ turbulent shear flow". In: Phys. Fluids 12.11 (2000), pp. 2976-2989. DOI: 10.1063/1.1313552.

[106] J. Schottler et al. "On the impact of non-Gaussian wind statistics on wind turbines - an experimental approach". In: Wind Energy Sci. 2.1 (2017), pp. 1-13. DOI: 10.5194/wes-2-1-2017.

[107] R. B. Cal et al. "Experimental study of the horizontally averaged flow structure in a model wind-turbine array boundary layer". In: J. Renew. Sustain. Energy 2.1 (2010). DOI: 10.1063/1.3289735.

[108] C. Küchler, G. Bewley, and E. Bodenschatz. "Experimental Study of the Bottleneck in Fully Developed Turbulence". In: J. Stat. Phys. 175·3-4 (May 2019), pp. 617-639. DoI: 10.1007/s10955-019-02251-1. 
[109] K. P. Griffin et al. "Control of long-range correlations in turbulence". In: Exp. Fluids 60.4 (Apr. 2019), p. 55. DOI: 10.1007/s00348-019-26981.

[110] A. S. Monin, A. M. Yaglom, and T. S. Lundgren. "Statistical Fluid Mechanics, Vol. II". In: J. Appl. Mech. 43.3 (Sept. 1976), pp. 521-521. DOI: $10.1115 / 1.3423915$.

[111] J. Jimenèz. "Turbulent velocity fluctuations need not be Gaussian". In: J. Fluid Mech. 376 (Dec. 1998), pp. 139-147. DOI: 10.1017/S0022112098002432.

[112] M. Wilczek, A. Daitche, and R. Friedrich. "Theory for the single-point velocity statistics of fully developed turbulence". In: Europhys. Lett. 93.3 (Feb. 2011), p. 34003. DOI: 10.1209/0295-5075/93/34003.

[113] M. R. Maxey. "The velocity skewness measured in grid turbulence". In: Phys. Fluids 30.4 (1987), p. 935. DOI: 10.1063/1.866279.

[114] H. Mouri et al. "Probability density function of turbulent velocity fluctuations". In: Phys. Rev. E 65.5 (May 2002), p. 056304. DoI: 10.1103/ PhysRevE. 65.056304.

[115] A. Vincent and M. Meneguzzi. "The spatial structure and statistical properties of homogeneous turbulence". In: J. Fluid Mech. 225 (1991), pp. 1-2O. DOI: 10.1017/S0022112091001957.

[116] P. A. Krogstad and P. A. Davidson. "Near-field investigation of turbulence produced by multi-scale grids". In: Phys. Fluids 24.3 (2012). DoI: $10.1063 / 1.3693132$.

[117] M. M. Metzger and J. C. Klewicki. "A comparative study of near-wall turbulence in high and low Reynolds number boundary layers". In: Phys. Fluids 13.3 (2001), p. 692. DOI: 10.1063/1.1344894.

[118] R. J. Hearst. "Fractal, Classical and Active Grid Turbulence From Production to Decay". PhD thesis. University of Toronto, 2015.

[119] G. H. Good and Z. Warhaft. "On the probability distribution function of the velocity field and its derivative in multi-scale turbulence". In: Phys. Fluids 23.9 (Sept. 2011), p. 095106. DoI: 10.1063/1.3632090.

[120] I. Hosokawa. "One-point velocity statistics in decaying homogeneous isotropic turbulence". In: Phys. Rev. E - Stat. Nonlinear, Soft Matter Phys. 78.6 (2008), pp. 1-6. DOI: 10.1103/PhysRevE.78.066312.

[121] P. G. Saffman. "The large-scale structure of homogeneous turbulence". In: J. Fluid Mech. 27.3 (1967), pp. 581-593. DOI: 10.1017/S0022112067000552. 
[122] P. G. Saffman. "Vortex dynamics". In: (1993), pp. 31-51.

[123] W. K. George. "The decay of homogeneous isotropic turbulence". In: Phys. Fluids A Fluid Dyn. 4.7 (July 1992), pp. 1492-1509. DoI: 10.1063/ 1.858423.

[124] W. K. George and H. Wang. "The exponential decay of homogeneous turbulence". In: Phys. Fluids 21.2 (Feb. 2009), p. 025108. Dor: 10.1063/ 1.3081557 .

[125] R. E. Seoud and J. C. Vassilicos. "Dissipation and decay of fractalgenerated turbulence". In: Phys. Fluids 19.10 (Oct. 2007), p. 105108. DoI: $10.1063 / 1.2795211$.

[126] G. Comte-Bellot and S. Corrsin. "The use of a contraction to improve the isotropy of grid-generated turbulence". In: J. Fluid Mech. (1966). DOI: $10.1017 / \mathrm{S} 0022112066000338$.

[127] L. Skrbek and S. R. Stalp. "On the decay of homogeneous isotropic turbulence". In: Phys. Fluids 12.8 (2000), pp. 1997-2019. DOI: 10.1063/ 1.870447 .

[128] P.-F. Yang, A. Pumir, and H. Xu. "Generalized self-similar spectrum and the effect of large-scale in decaying homogeneous isotropic turbulence". In: New J. Phys. 20.10 (Oct. 2018), p. 103035. DOI: 10.1088/13672630/aae72d.

[129] M. Oberlack. "On the decay exponent of isotropic turbulence". In: PAMM 1.1 (Mar. 2002), p. 294. DOI: 10 . 1002/1617-7061(200203) 1 : 1<294: : AID-PAMM294>3.0.CO;2-W.

[130] C. Tropea, A. L. Yarin, and J. F. Foss, eds. Springer Handbook of Experimental Fluid Mechanics. Berlin, Heidelberg: Springer Berlin Heidelberg, 2007. DOI: 10 . 1007/978-3-540-30299-5.

[131] M. K. Fu, Y. Fan, and M. Hultmark. "Design and validation of a nanoscale cross-wire probe (X-NSTAP)". In: Exp. Fluids 6o.6 (June 2019), p. 99. DOI: $10.1007 / \mathrm{s} 00348-019-2743-0$.

[132] F. E. Jørgensen. How to measure turbulence with hot-wire anemometers - a practical guide. Skovlunde, Denmark: Dantec Dynamics A/S, 2002.

[133] K. Griffin et al. "Correlated forcing with an active grid to control turbulence". In: August (2016), pp. 26-27. 
[134] K. Chang, G. P. Bewley, and E. Bodenschatz. “Experimental study of the influence of anisotropy on the inertial scales of turbulence". In: $J$. Fluid Mech. 692 (Feb. 2012), pp. 464-481. DoI: 10.1017/jfm. 2011.529. 


\section{Part II}

\section{Eulerian Measurements}





\section{Chapter 5}

\section{On Hot Wire Measurements}

This chapter serves three purposes: (i) introduction of the measurement principle used in this part of the thesis (ii) verify its applicability to the flow under study, and (iii) report on progress regarding the technique that has been used in the VDTT. First, a general introduction to the measurement principle is provided loosely following Ch. 5.2 of [1]. Then, the limiting timeand length scales of the system introduced by the sensing element are given for the conditions in the VDTT. Afterwards, two main circuits are introduced and some new insights into their operation with nanoscale sensors are presented. Next, the validity of Taylor's Hypothesis is studied for measurements at turbulence intensities $>10 \%$ (typical for active grid turbulence). Finally, the hot wire measurement protocol used here is described along with routine calculations and a comparison of different ways to determine the turbulent dissipation rate $\varepsilon$.

\subsection{Introduction to Hot Wire Anemometry}

\subsubsection{Operating Principle and King's Law}

Hot wire anemometry is a technique to measure the local fluid velocity through the flow's advective cooling. The electric resistance of a conducting wire depends in many cases on the temperature of the wire material. Typically, a higher temperature corresponds to a higher electrical resistance (positive temperature coefficient of resistivity). This phenomenon is exploited to transform a temperature signal into a voltage signal. For example, PT1oo elements that are commonly used for a rough temperature measurement are simply a platinum film with a resistance of $100 \Omega$ at $20^{\circ} \mathrm{C}$.

In hot wire anemometry this physical phenomenon is applied to the mea- 


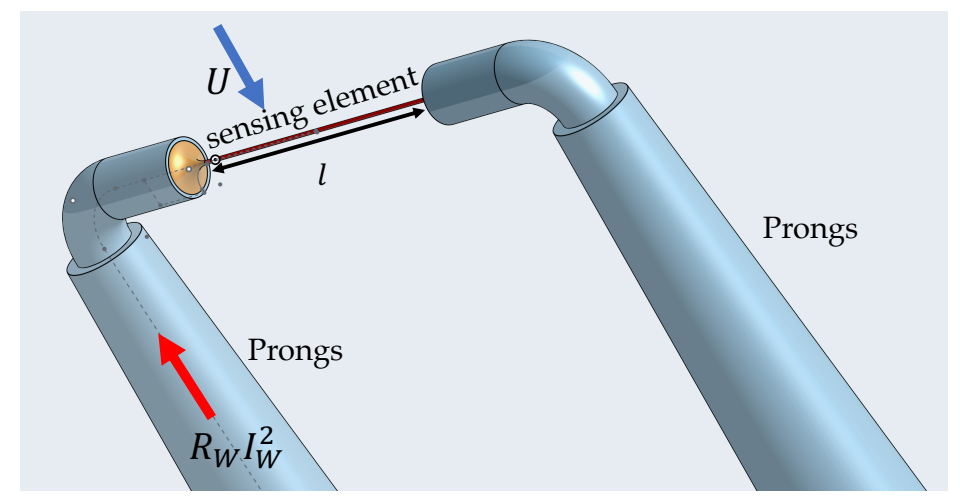

Figure 5.1: Illustration of the hot wire measurement principle. The ohmic heating $R_{W} I_{W}^{2}$ heats the wire to its operating temperature $T_{W}$. It is cooled by a flow of velocity $U$. This causes a change in the wire resistance, which can be picked up by connected electronics.

surement of flow velocities. The idea is that a fluid flow over a material carries away excess heat it might have compared to the environment and thus causes a change in the material's temperature (forced convective cooling). It is clear that a direct change of the temperature in the surrounding fluid has the same effect and pollutes the velocity measurement. To make this effect negligible, the wire is operated at a temperature $T_{W}$ significantly higher than the ambient temperature $T_{a}\left(T_{W}-T_{a}>100^{\circ} \mathrm{C}\right)$.

The Nusselt number $\mathrm{Nu}$ is the dimensionless number that characterises the relative importance of convective over conductive heat transport and therefore allows insights into the effect of convective cooling of a heated wire of diameter $d$ and length $l$. It is typically defined as

$$
\mathrm{Nu}=\frac{h L}{k} .
$$

$k$ is the thermal conductivity of the fluid, and $L$ is a characteristic length scale (in this case the wire diameter $d$ ). $h$ is the convective heat transfer coefficient, which quantifies the heat flux through a surface of area $A=\pi l d$ given a temperature difference $T_{W}-T_{a}$ across the surface. The heat flux $d Q / d t$ is given by the ohmic heating $R_{W} I_{W}^{2}$, necessary to maintain the wire temperature. The Nusselt number can therefore be reexpressed as

$$
\mathrm{Nu}=\frac{R_{W} I_{W}^{2}}{\pi l k\left(T_{W}-T_{a}\right)} .
$$

The wire resistance is assumed to be a linear function of its temperature, i.e. $T_{W}-T_{a}=\left(R_{W}-R_{a}\right) /\left(\chi R_{a}\right)$, where $R_{a}$ is the wire temperature in quiet fluid, and $\chi$ the temperature coefficient of resistivity of the wire material. 
The Nusselt number dependence on the wire Reynolds number Re has been investigated empirically (see [2] for a list of references). The one commonly used for hot wire anemometry reads $\mathrm{Nu}=A+B(\operatorname{Re})^{n}$. Setting equal the wire Nusselt number and (5.2), one arrives at King's Law

$$
V^{2}=A^{\prime}+B^{\prime} U^{n}
$$

Here, $I_{W}^{2} \sim V_{W}^{2}$ was used, and the fluid and material coefficients were absorbed into the coefficients $A^{\prime}$, and $B^{\prime} . n$ is around 0.50 for conventional wires, but can be higher for film-like miniature wires [3].

\subsubsection{Wire Requirements}

The sensing element is naturally an essential factor for the performance of any hot wire system. Ideally, its thermal coefficient of resistance $\chi$ is large, and its thermal conductivity low to ensure a good sensitivity and quick response to changes in the flow velocity. Nickel, Silver, and Platinum are regularly used to build hot wire sensors [1].

The length of a hot wire determines its spatial resolution, which is ideally $\leq \eta$. However, end conduction effects limit the ratio of wire length $l$ to its diameter $d$. A common rule of thumb is $l / d>200$, which was recently revisited by Hultmark et al. [4]. Still, shorter wires must be thinner and thus more delicate to operate.

Since $\eta$ can be as small as $10 \mu \mathrm{m}$ in the VDTT, experiments in this facility require the use of the smallest sensors available. These are currently the so-called Nanoscale Thermal Anemometry Probes (NSTAPs) produced by Princeton University $[5,6,7]$. An alternative was developed recently by Twente University [8]. While the NSTAP sensing element is technically a film of dimensions $30 / 60 \mu \mathrm{m} \times 2.5 \mu \mathrm{m} \times 1$ 10onm, the Twente wire is an almost cylindrical sensor of similar length.

Unless stated otherwise, the experiments in this part of the thesis were conducted with $30 \mu \mathrm{m}$ NSTAP or a conventional platinum wire of $450 \mu \mathrm{m}$ length and $2.5 \mu \mathrm{m}$ diameter. These probes are well-established and the reader is referred to the literature $[5,9,10,11,12,13,14,7,15]$ for the details.

Particular to the flow under study are the extremely high Reynolds numbers, the operating gas, and the associated small Kolmogorov scales $\eta$. Such environments are similar to those found in liquid helium, where the thermal boundary layer around a probe can significantly impact the measurements of small-scale turbulence $[16,17,18]$. This viscous boundary layer does not 


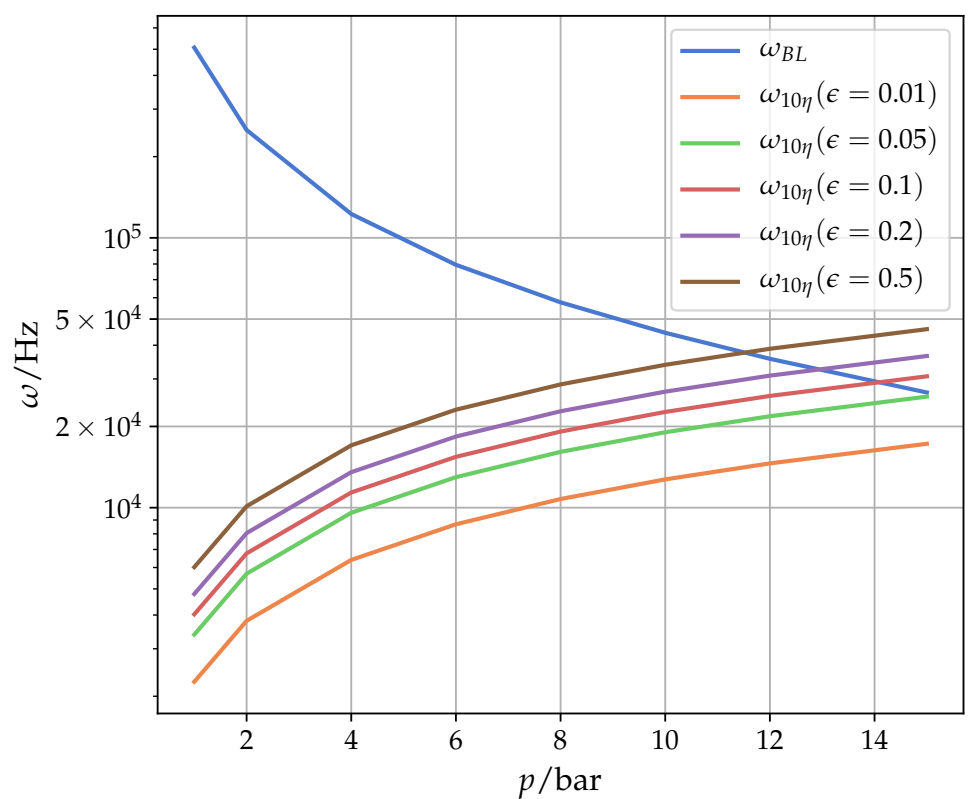

Figure 5.2: Critical Frequency $\omega_{B L}=\kappa / d^{2}$ induced by the presence of a viscous boundary layer through which the temperature differences between wire and flow must diffuse. For comparison, the experimental frequency where $10 \eta$ are typically found is indicated for different values of the turbulent dissipation rate $\varepsilon$.

follow the flow and any temperature difference between the flow and the wire must diffuse through it. Considering the Reynolds number based on the probe diameter $d$, it is found that the relevant boundary layer size scales with d. Following the arguments of Emsellem et al. [16], the thermal boundary layer affects the flows time scales faster than the diffusive time scale through the boundary layer

$$
\tau_{B L}=d^{2} / \kappa,
$$

where $\kappa$ is the thermal conductivity of the material. This time scale induces a critical frequency $\omega_{B L}=1 / \tau_{B L}$, which is shown in Fig. 5.2 as a function of the ambient pressure. The highest frequencies encountered in the experiments presented here are given by the Kolmogorov scale $\eta$, which is swept by the sensor at the speed of the mean flow $U$ in the most demanding cases. For $U=4.5 \mathrm{~m} / \mathrm{s}$ the experimental frequency where $10 \eta$ would be measured is shown in Fig. 5.2 as well. At high pressures and for large dissipation rates $\varepsilon$, this frequency exceeds that of the thermal boundary layer and the statistics may therefore be influenced by the viscous boundary layer around the probe. It is however clear that in the inertial range, which starts around $100 \eta$, the thermal boundary layer around the probe does not play a role. 


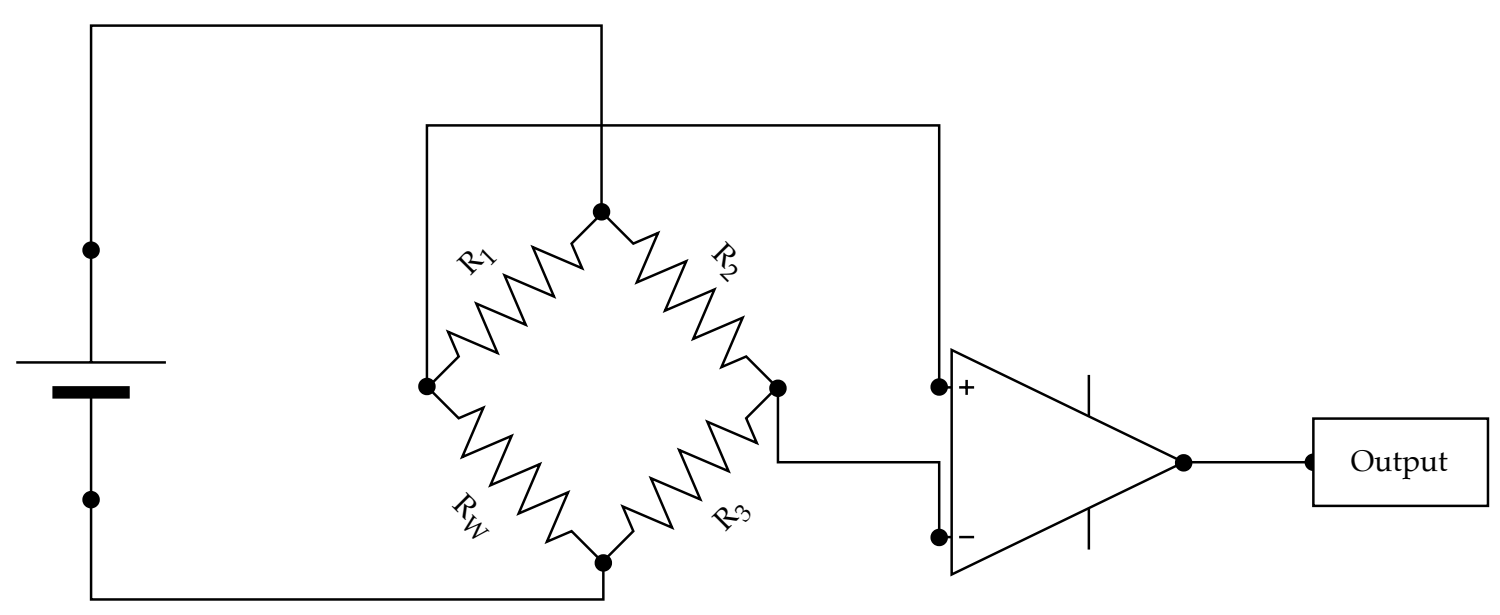

Figure 5.3: Example for a simple constant current anemometer. The Wheatstone bridge improves the noise characteristics if the potentiometer is used to balance the bridge. To compensate for the time constant of the wire, a $R C$-circuit can be placed between the bridge and the output amplifier.

\subsubsection{Anemometry Circuits}

There exist three common methods to drive hot wires, each with intrinsic advantages and disadvantages. The reader is referred to the review in Ref. [1]. An overview is provided in the following paragraphs along with results on the frequency response of the system used in this thesis. These results are complemented by the discussion found in Ch. 9 .

Constant Current Anemometer (CCA) The simplest form of the CCA consists of a high-precision current source or a low-noise voltage source with a large resistor whose output is driven through the wire and the voltage drop across it is measured. The main attractiveness of the circuit lies in its simplicity and low costs. For conventional hot wires it will result in a poor frequency response, high noise, and small sensitivity towards flow changes. Some of these problems can be reduced by electronic correction (e.g. [19]) and placement of a wire in a Wheatstone bridge. A basic circuit without frequency compensation is shown in Fig. 5.3.

As the wire becomes smaller and smaller, i.e. has a smaller and smaller thermal mass, these problems also gradually disappear. The time constant of the wire decreases, because the thermal equilibrium after an initial change in wind speed is restored quicker and quicker. This can become particularly relevant when using MEMS-manufactured nanowires, such as the NSTAP [5, 9, 7] or similar approaches [8,3]. Such wires are notoriously difficult to operate and are known to cause instabilities in conventional CTA circuits, which 

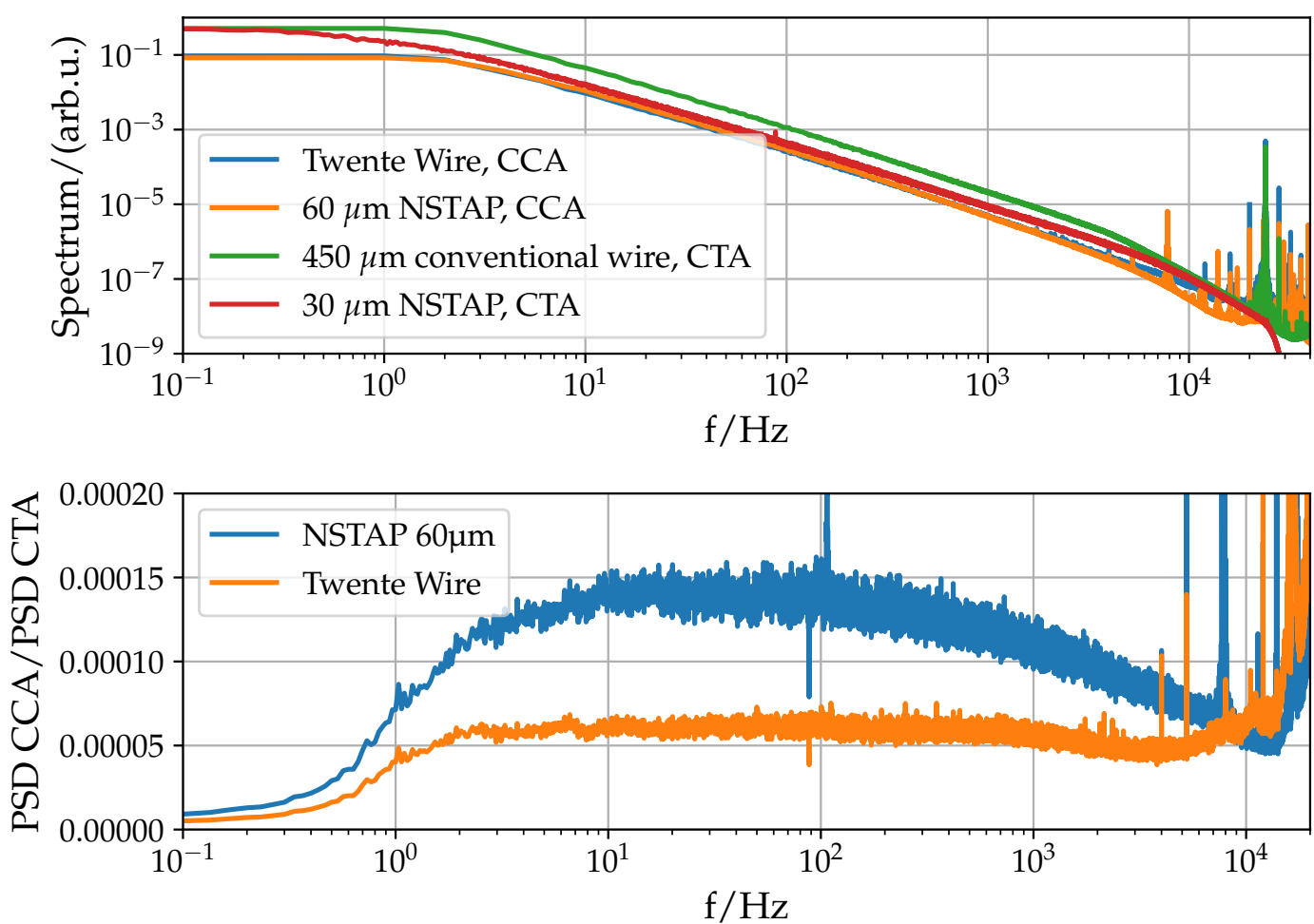

Figure 5.4: Upper plot: comparison of different power spectra recorded with different combinations of sensing wire and anemometer circuit under similar flow conditions. The CCAoperated wires start to deviate from the CTA curve around $1 \mathrm{kHz}$. Lower Plot: Comparison of the performance of two different micromachined wires using the same CCA bridge (PSD CCA) using a CTA measurement with an NSTAP as benchmark (PSD CTA). While the cylindrical Twente wire follows the CTA measurement fairly well, the NSTAP response differs significantly.

leads to fatal wire burning [7]. The CCA provides a way to operate the wire in a very controlled way allowing a slow increase in heating current free of feedback-loops. Although these wires have a respectable frequency response even when operated with a very simple CCA, the system cannot take advantage of the wire's fine spatial resolution, because substantial damping occurs for frequencies above $1 \mathrm{kHz}$ as can be seen in Fig. 5.4. The commercial CTA circuit can be tuned to optimise the frequency response using a square wave test, which corrects for the wire thermal lag. In the CCA system as implemented here, the finite wire response is not compensated and completely dictates the system frequency response.

Here, the spectra of a $60 \mu \mathrm{m}$ long NSTAP and a "Twente Wire" of similar length (both with overheat ratio 1.18) are compared to a $30 \mu \mathrm{m}$ long NSTAP driven by a commercial Dantec StreamWare Constant Temperature Anemometer. The main difference between the NSTAP and the Twente Wire is their cross-section. While the NSTAP is strictly speaking a film $100 \mathrm{~nm}$ thick- 


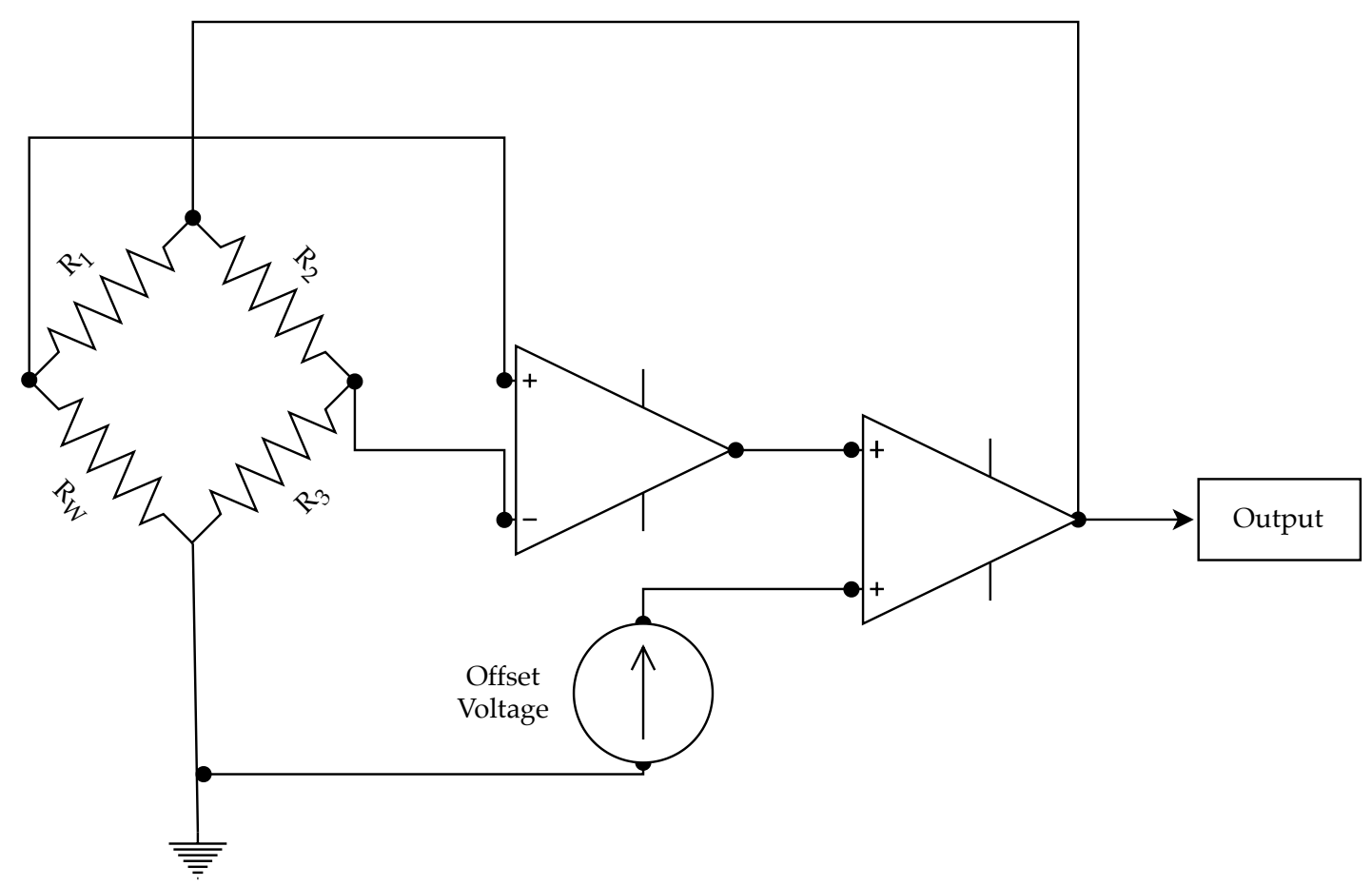

Figure 5.5: Schematic of a simple CTA circuit [1]. The feedback loop corrects any imbalance in the otherwise balanced bridge, such that the wire resistance $R_{W}$ remains constant. Typically, $R_{1}=R_{2}$, and $R_{3}$ is a variable resistor

ness and $2.5 \mu \mathrm{m}$ width, the Twente Wire is close to a round cylinder [8]. It is apparent that both CCA-spectra cut the spectrum off significantly compared to a CTA. Building the ratio between the two CCA spectra and the CTA spectrum reveals that the Twente Wire is essentially parallel to the CTA curve up to the cutoff frequency. The 6o $\mu \mathrm{m}$ NSTAP driven with a CCA has a less constant frequency response. In any case, the nanoscale sensor cannot overcome the disadvantages of the circuit and a frequency-dependent compensation is required for them to compete with constant temperature anemometers.

Constant Temperature Anemometer (CTA) The CTA has been the most widely used hot wire anemometer for decades, because it provides a very good frequency response, excellent noise characteristics and allows measurements at a constant wire temperature. The principle is based on a feedback loop that corrects any change in wire resistance by increasing or decreasing the current through the wire. A simplified example circuit is shown in 5.5.

Because the wire is immediately driven back to its nominal overheat, the thermal lag of the wire is reduced significantly leading to a superior frequency response over CCA or constant voltage anemometry (CVA). This comes at the expense of a more complex system. While CCA and CVA are linear systems 


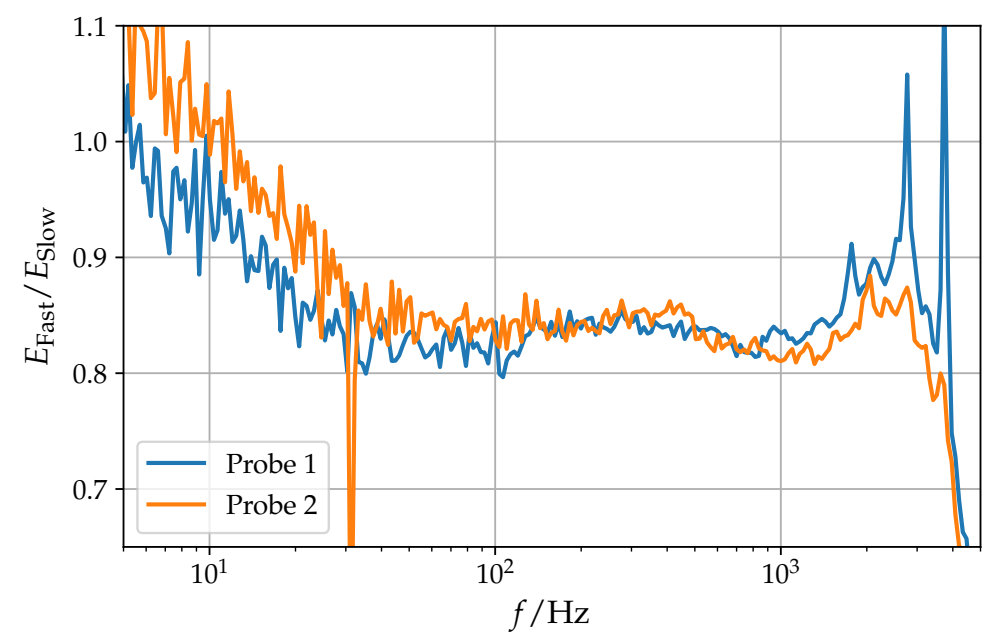

Figure 5.6: Measured frequency response using a CTA with two different NSTAPs. Spectra at equal $R_{\lambda}$, but different frequency contents are compared. Both show a dampening between 0.5 and $1 \mathrm{kHz}$ and a subsequent amplification of the signal. The effects are small, but significant. There appears to be a relatively small probe-to-probe variance.

that are easy to analyse, the CTA is a nonlinear system. In a linearized form its theory has been studied extensively $[20,21,10]$. It has been found that a CTA performance is very sensitive to all parts of the system, including cable inductances and probe support resistance $[1,22]$. While the frequency response of a CCA or CVA is essentially that of a low-pass filter (provided no frequency compensation has been added), the response of a CTA is more complex. Unexpected damping or amplification effects can occur at rather low frequencies, in particular when using nanoscale probes [10, 23].

For example, Hutchins et al. [23] have compared spectra of identical Reynolds numbers, but different frequency contents with combination of an NSTAP and a Dantec StreamWare CTA (the same system used for most measurements presented in this thesis). They concluded that this system exhibits a damping of up to $10 \%$ starting around $500 \mathrm{~Hz}$. This is a substantial effect when small features in scale-resolved statistics are investigated (see also Ch. 9). Their measurements have been performed in a high pressure pipe flow. By changing both the kinematic viscosity through the fluid density and the mean flow speed they can create flows at practically identical Reynolds numbers, very different frequency ranges. Comparison of these features then yields a measure of the damping at a given frequency. In the VDTT such measurements can be performed at constant pressures, by varying turbulence intensity through the active grid and mean flow speed at the same time. It is assumed that at low frequencies the frequency response curve is essentially 
flat, and at the more interesting higher frequencies, the details of the turbulence generation play only a minor role. One therefore measures a velocity spectrum once at small wind speeds, but high grid solidity (active grid flaps at $70^{\circ}$ ) and once at high mean flow speed, but small grid solidity (active grid flaps at $10^{\circ}$ ). This method presented essentially confirms the results of Ref. [23] as shown in Fig. 5.6. The signal is dampened between $500 \mathrm{~Hz}$ and $1 \mathrm{kHz}$, then amplified up to around $3 \mathrm{kHz}$. Beyond $3 \mathrm{kHz}$ definite conclusions are difficult, because the curve is very sensitive to the frequency normalisation.

Alternative methods of obtaining the frequency response are electrical heating through square- or sine waves [24], laser heating of the wire[25], and fluid 'square waves' [26]. Square waves were routinely used in this study to optimise the frequency response (see Sec. 5.3.1).

In combination, Figs. 5.4 and 5.6 show that the Dantec StreamWare CTA is far superior over the in-house CCA, but its frequency response is still far from ideal. Furthermore, the tendency of the CTA to break wires oftentimes prevents the consistent use of a specific hot wire for many measurements. A frequency-compensation of the CCA or the design of a constant voltage anemometer should be considered to allow high-fidelity hot wire measurements optimised for miniature hot wires and with a high degree of control. The use of the constant tempertaure system considered here requires great care in the interpretation of data. It is an important asset of the VDTT that similar Reynolds numbers can be created in many different ways unless the Reynolds number is extremely high. This provides an important means of distinguishing turbulence phenomena from probe effects.

\subsection{Taylor's Hypothesis}

\subsubsection{Random Sweeping Hypothesis and One-Dimensional Spectra (joint project with M. Wilczek)}

The aim of the hot wire measurements presented here is to obtain statistics about the turbulent flow field that passes by the sensor, i.e. a spatially resolved measurement of flow velocities. However, a single hot wire can only provide time series of flow velocities $U(t)$. These time series are commonly converted into one-dimensional flow fields $U(x)$ by the following approximation first stated by Taylor in 1938 [27]: In situations where the mean flow $\langle\mathbf{U}\rangle$ is relatively constant and unidirectional compared to its fluctuations 
$u(t)=U(t)-\langle U(t)\rangle$, i.e. where $\sqrt{\left\langle u(t)^{2}\right\rangle} \ll\langle U\rangle$, the fluctuating part of the velocity signal is passively advected past the sensor by the mean flow the turbulent field is 'frozen' and carried over the sensor. Then time $t$ can be replaced by $x=\langle U\rangle t$. For the remainder of this part of the thesis, only the fluctuating part of the velocity signal will be of importance and $\langle U\rangle$ is denoted as $U$ for simplicity.

Taylor's assumption is a fundamental part of almost all studies of turbulence statistics that rely on one-point measurements, such as Laser-Doppler anemometry or single hot wire anemometry. In this section, the validity of Taylor's Hypothesis is critically assessed by comparing it to the alternative concept of a turbulence field that is advected past the sensor by a sweeping velocity $V$, which is a random variable with a Gaussian distribution. This will yield an estimate of the potential error due the imperfections of Taylor's Hypothesis.

The hypothesis of large-scale eddies carrying the turbulent velocity field was first introduced by Kraichnan [28] and Tennekes [29]. The corresponding change of variables is $\mathbf{u}(\mathbf{x}) \rightarrow \mathbf{u}((\mathbf{U}+\mathbf{V}) t)$, which leads to the differential equation

$$
\frac{d \boldsymbol{u}}{d t}=-(\boldsymbol{U}+\boldsymbol{V}) \cdot(\nabla \cdot \mathbf{u})
$$

In fourier space this gives [28]

$$
\frac{d \boldsymbol{u}(\boldsymbol{k}, t)}{d t}=-i \boldsymbol{k} \cdot(\boldsymbol{U}+\boldsymbol{V}) \boldsymbol{u}(\boldsymbol{k}, t)
$$

which can be solved exactly:

$$
\boldsymbol{u}(\boldsymbol{k}, t)=\boldsymbol{u}(\mathbf{k}, 0) \exp [\boldsymbol{k} \cdot(\boldsymbol{U}+\boldsymbol{V}) t]
$$

It can then be shown [30, 31], that the wavenumber-period spectrum is related to the wavenumber spectrum by

$$
E(\boldsymbol{k}, t)=E(\boldsymbol{k})\langle\exp [-i \boldsymbol{k} \cdot(\boldsymbol{U}+\boldsymbol{V}) t]\rangle
$$

Assuming a Gaussian distribution of large-scale sweeping velocities of mean $\boldsymbol{U}$ and variance $\boldsymbol{V}$ we obtain after a fourier transform the wavenumberfrequency spectrum

$$
E(\boldsymbol{k}, \omega)=\frac{E(\boldsymbol{k})}{\sqrt{2 \pi k^{2} V^{2}}} \exp \left[-\frac{(\omega-\boldsymbol{k} \cdot \boldsymbol{U})^{2}}{2 k^{2} V^{2}}\right]
$$


The full spectral tensor of the velocity field $\Phi_{i j}(\boldsymbol{k})$ contains information about the correlations between velocity components $u_{i}$ and $u_{j}$ separated by the wavevector $\boldsymbol{k}$. The measurement of a single hot wire will yield the onedimensional spectrum in longitudinal direction [32] $E_{11}\left(k_{1}\right)=2 \int_{-\infty}^{\infty} \int \Phi_{11} d k_{2} d k_{3}$. Under the assumption of statistical isotropy and homogeneity the full spectral tensor can be reduced to the energy spectrum function (energy spectrum) $E(k)(k=|\boldsymbol{k}|)$, to which it is related by

$$
\Phi_{i j}(\boldsymbol{k})=\frac{E(k)}{4 \pi k^{2}}\left[\delta_{i j}-\frac{k_{i} k_{j}}{k^{2}}\right] .
$$

Therefore, the one-dimensional longitudinal wavenumber spectrum is given by [33] (see also 3.1.2)

$$
E_{11}\left(k_{1}\right)=\int_{0}^{\infty} \frac{E(k)}{4 \pi k^{2}}\left(1-\frac{k_{1}^{2}}{k^{2}}\right) d k
$$

in isotropic and homogeneous turbulence. The one-dimensional frequency spectrum that is actually measurable by the hot wire can then be obtained by eliminating all directional information. Using the Gaussian sweeping model eq. 5.9 one obtains

$$
\begin{aligned}
E_{11}(\omega) & =\int_{0}^{\infty} k^{2} d k \int_{0}^{2 \pi} d \phi \int_{0}^{\pi} \sin (\theta) d \theta \frac{E(k)}{4 \pi k^{2}}\left(1-\frac{k_{1}^{2}}{k^{2}}\right) \frac{1}{\sqrt{2 \pi k^{2} V^{2}}} \exp \left(-\frac{(\omega-\boldsymbol{k} \cdot \boldsymbol{U})^{2}}{2 k^{2} V^{2}}\right) \\
& =\int_{0}^{\infty} k^{2} d k \int_{0}^{2 \pi} d \phi \int_{0}^{\pi} \sin (\theta) d \theta \frac{E(k)}{4 \pi k^{2}}\left(1-\cos ^{2}(\theta)\right) \frac{1}{\sqrt{2 \pi k^{2} V^{2}}} \\
& =\int_{0}^{\infty} d k \int_{0}^{\pi} d \theta \frac{E(k)}{\sqrt{8 \pi k^{2} V^{2}}} \sin ^{3}(\theta) \exp \left(-\frac{(\omega-k U \cos (\theta))^{2}}{2 k^{2} V^{2}}\right)
\end{aligned}
$$

Evidently, under random sweeping all $k$ contribute to the frequency spectrum through a weighting function. This weighting function is illustrated in Fig. 5.7. At a given frequency $\omega_{p}$, points that correspond to a wavenumber about $0.2 \omega_{p}$ contribute strongest to $E\left(\omega_{p}\right)$. In the following, we replace $E(k)$ in eq. (5.14) by different model spectra. 


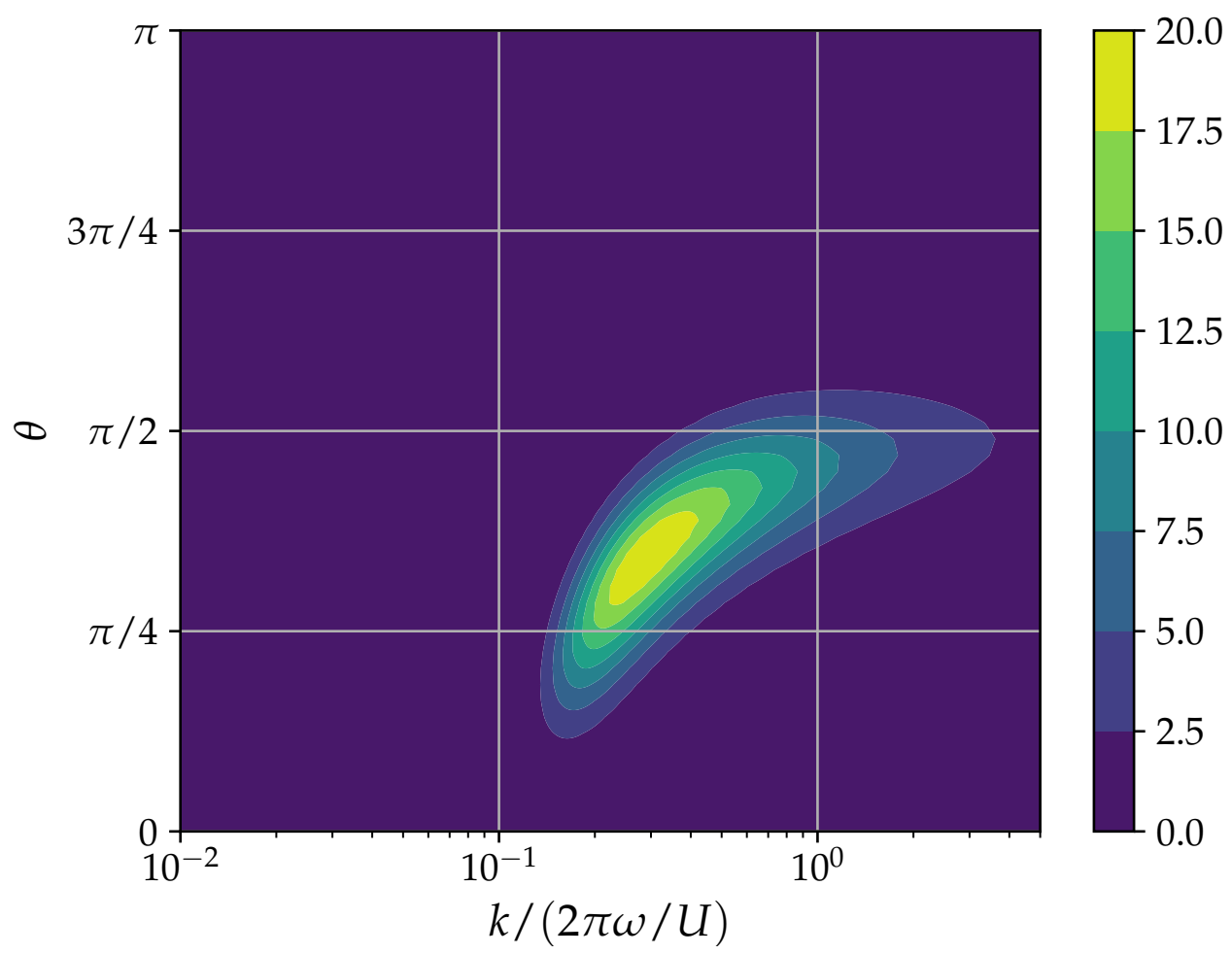

Figure 5.7: The weighting function $\frac{1}{\sqrt{8 \pi k^{2} V^{2}}} \sin ^{3}(\theta) \exp \left(-\frac{(\omega-k U \cos (\theta))^{2}}{2 k^{2} V^{2}}\right)$ that determines the contributions of $k$ and $\omega$ to the one-dimensional frequency spectrum $E(k)$.

\subsubsection{Application to Model Spectra}

The energy spectrum $E(k)$ follows a power law with an exponent close to $5 / 3$ in the inertial range. Wilczek et al. [34] have shown that the application of a random sweeping model (5.14) sustains such a power law. Therefore, Taylor's hypothesis can be applied up to very high turbulence intensities $V / U$ to obtain the value of the spectral exponent. They found however, that the value of the Kolmogorov constant $C_{K}$ is impacted by random sweeping and predict that spectral features such as the small- and large-scale cutoffs or the bottleneck effect are smeared out by the presence of large-scale random sweeping. Here model spectra are used in place of $E(k)$ in eq. (5.14) to elucidate the relevance of these effects for turbulence measurements in a wind tunnel with the increased turbulence intensities introduced by an active grid. To simplify the notation, without loss of generality, $U=1$ is chosen.

$k^{-5 / 3}$-spectrum with cutoffs To explore the effects of random sweeping in the most simple way, we consider a $k^{-5 / 3}$-Spectrum with sharp cutoffs at 


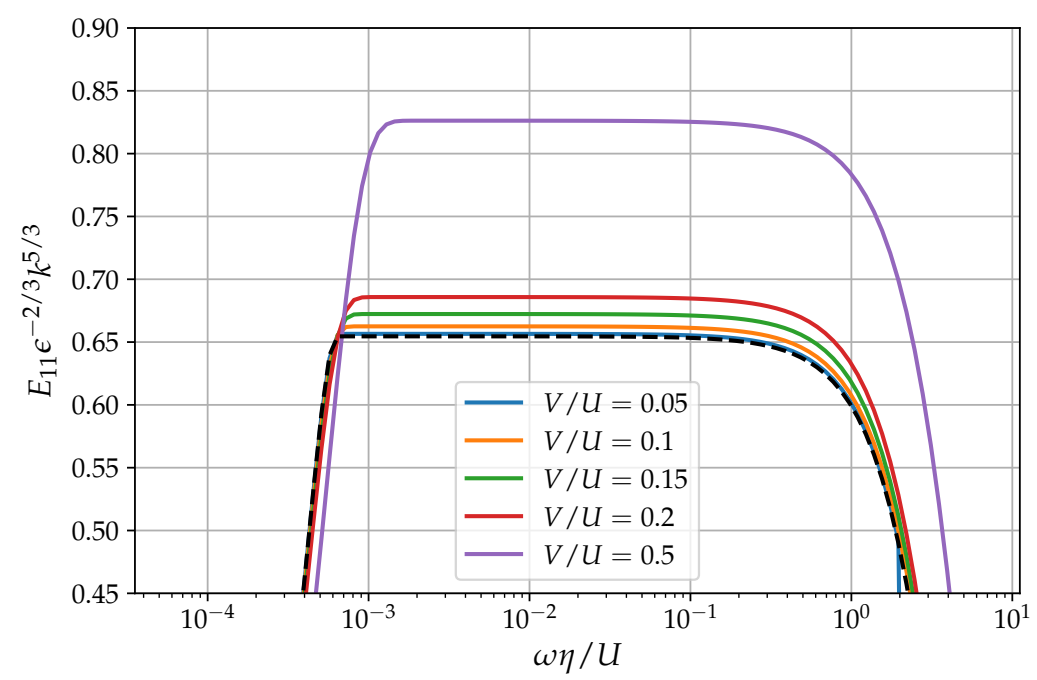

Figure 5.8: Compensated one-dimensional spectra based on a $k^{-5 / 3}$-spectrum with cutoffs at small and large scales. The sharp cutoffs are smeared out by the projection on the single component of the spectral tensor (dashed curve). The effects of the random advection velocity $V$ cause additional smoothing of these edges and their apparent shift towards higher frequencies. The Kolmogorov constant $C_{K}$ is increasingly overestimated with increasing $V$.

scales $\eta$ and $L$ :

$$
E(k)= \begin{cases}k^{-5 / 3}, & \text { for } 2 \pi / L<k<2 \pi / \eta \\ 0, & \text { else }\end{cases}
$$

This "spectrum" is inserted into eq. (5.14) and the integrals evaluated numerically using dblquad from scipy. integrate. The integration is carried out for different values of $V$ while keeping $\eta$ (and thus $L$ ) constant. $\varepsilon=0.5 V^{3} / L$, and the kinematic viscosity $v=\left(\eta^{4} \varepsilon\right)^{1 / 3}$ are adjusted in such a way that the Reynolds number $R_{\lambda}=3000$ is the same for all cases. Fig. 5.8 shows the spectra compensated by the Kolmogorov prediction and compares them to the case $V / U \rightarrow 0$. The flat segment is located at the level of the resulting Kolmogorov constant $C_{K}$ and clearly rises with increasing sweeping velocity $V$. The sharp edges of the original $E(k)$ are rounded off by both the conversion to $E_{11}$, and the random sweeping effects. Furthermore, the apparent position of these cutoffs is shifted towards higher frequencies, which can be anticipated from the random-sweeping term in the integrand. The example in Fig. 5.7 shows that the maximum contribution to $E(\omega)$ occurs a wavenumbers $<2 \pi \omega$. Thus, features at a given $k$ in the original one-dimensional spectrum calculated by (5.11) are shifted towards larger frequencies. 

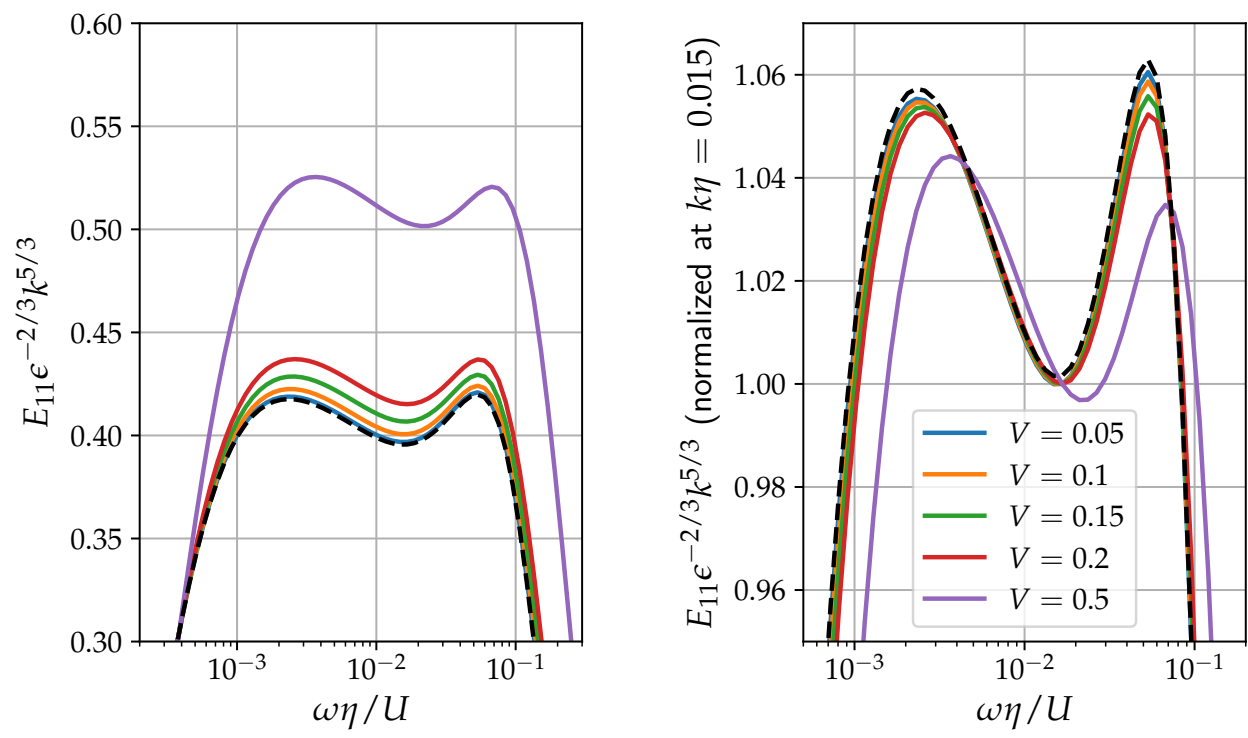

Figure 5.9: Spectra resulting from the random sweeping model to the comprehensive model spectrum by Meyers \& Meneveau [35] compensated by the Kolmogorov prediction. Dotted black lines denote the one-dimensional spectrum in the limit $V \rightarrow 0$, i.e. when Taylor's Hypothesis is prefectly valid. Left: The level of the inertial range rises as a result of random sweeping. Right: The same curves are collapsed at $\omega \eta / U=0.015$, which corresponds approximately to the position of the minimum in the inertial range. Features such as the bottleneck effect are attenuated by the random sweeping model. The spectra are also shifted towards higher frequencies. The effect is rather small for turbulence intensities occuring in active grid turbulence.

Model Spectrum by Meyers \& Meneveau (2008) There exists a long tradition of attempts to provide functional forms of scale-resolved statistical quantitites such as the energy spectrum function $E(k)$ that effectively describes the data obtained from measurements and numerical simulations. An early example for the second order structure function was given by Batchelor [36], which was later transformed into spectral space [37]. Pope [32] provided a spectral form with more parameters by combining a spectrum of exponent $5 / 3+\beta$ and functional forms of he large- and small-scale ends of the spectrum. Meyers \& Meneveau [35] use a similar approach, but also parameterise the the bump at the transition between inertial and disspation range (bottleneck effect). Their model is further consistent with fundamental physical 
relations, such as $u_{R M S}^{2}=V^{2} \sim \int E(k) d k$. The full model reads

$$
\begin{aligned}
E(k) & =\frac{55}{18} C_{K} \varepsilon^{2 / 3} k^{-5 / 3}(k L)^{-\beta} f_{L}(k L) f_{\eta}(k \eta) \\
f_{L}(k L) & =\left\{\frac{k L}{\left[(k L)^{p}+\alpha_{5}\right]^{1 / p}}\right\}^{5 / 3+\beta+2} \\
f_{\eta}(k \eta) & =\exp \left(-\alpha_{1} k \eta\right)\left[1+\frac{\alpha_{2}\left(k \eta / \alpha_{4}\right)^{\alpha_{3}}}{1+\left(k \eta / \alpha_{4}\right)^{\alpha_{3}}}\right]
\end{aligned}
$$

This model is implemented into the random sweeping framework with $R_{\lambda}=$ 3000 , and $\eta=0.01$ for different $V$ and the corresponding values of $L, \eta$, and $\varepsilon$. Fig. 5.9 shows that the random sweeping has three main effects on the compensated spectra: First, the apparent Kolmogorov constant, i.e. the level of the inertial range in compensated plots, increases with increasing sweeping velocity. This is in agreement with earlier findings [34]. Second, the positions of distinct features, such as the bottleneck effect, are shifted towards higher frequencies. Third, the random sweeping causes an attenuation of these features depending on the sweeping velocity. These effects are rather weak when $V / U<0.2$, in accordance with the empirical rule-of-thumb that the turbulence intensity must not exceed $20 \%$ for Taylor's Hypothesis to be applicable. In the VDTT with the active grid turbulence intensities can reach up to $15 \%$. Therefore, some care must be taken when studying the fine details of spectra. Conclusions from measurements at very high turbulence intensities should be cross-checked with measurements at lower intensities.

\subsection{Measurement Protocol and Data Analysis}

The previous sections have been concerned with some potential sources for systematic errors when acquiring (scale-resolved) measurements of Eulerian statistics using hot wire anemometry. Naturally, the particular difficulties within the VDTT environment were emphasised. Since these error sources span all ranges of scales, their systematic elimination is practically impossible. The overall strategy for acquiring the data was therefore to generate dataset that scan the available range of Reynolds numbers in different ways whenever possible. This way, observations that are not robust against different probes, values of $\eta$, flow speeds, or turbulence intensity can be identified. This section will introduce the hot wire system used in this thesis, the data analysis algorithm applied routinely to any dataset, and will present different ways of estimating the turbulent dissipation rate $\varepsilon$. 


\subsubsection{Calibration and Data Acquisition}

The hot wire measurements presented here were recorded using either Nanoscale Thermal Anemometry Probes (NSTAPs) $[5,6,7]$ provided by Princeton University or $450 \mu \mathrm{m}$ long conventional Dantec Hot Wires. The wires were mounted on the traverse depicted in Fig. 4.2 between 0.45 and $0.65 \mathrm{~m}$ from the measurement section floor and less than $10 \mathrm{~cm}$ away from its center. The sensors were connected by $20 \mathrm{~m}$ long RG223 BNC cables through NPT cable feedthroughs to a Dantec StreamWare Constant Temperature Anemometer.

In the case of the NSTAPs the protocol recommended by Princeton University [38] was followed. For this, an external resistor $R_{\text {ext }}$ was included in the bridge. Since the CTA feedback attempts to maintain a zero potential difference between the arms of its bridge and its two top resistors are identical, the value of this resistor is equal to the wire's (hot) resistance. The wire overheat is therefore determined by $a_{W}=R_{\text {ext }} / R_{\text {cold }}$, where $R_{\text {cold }}$ is the resistance of the wire measured in its unheated state. Typical values for $a_{W}$ were 1.2-1.3, i.e. slightly lower than those reported by Princeton University. It was essential to bypass all automatic overheat settings provided by the system to avoid wire burning.

The overheat ratio of the conventional wires could be automatically set to 1.8 by the system in all cases.

After setting the overheat, a square wave test was performed and its response was optimised. After conditioning the signal to fall within the bounds of the data acquisition system, the hot wires were ready to be calibrated.

Calibration and data acquisition were controlled by a MATLAB program. To calibrate the wires, the flow velocity was measured directly by means of a pitot tube and a static pressure tube attached to the two ports of a SITRANS DS-PIII differential pressure transducer outside the wind tunnel. The pressure transducer was referenced by applying zero pressure difference at the beginning of each measurement day. Its output (4-20mA) was calibrated to the voltage measurement of the data acquisition system before each calibration. Mean CTA output and differential pressure were recorded for 60 seconds for 15-30 different flow speeds by incrementally changing the wind tunnel fan rotation speed. In between measurements the flow was allowed to homogenise for 45 seconds. The active grid was fully opened during calibration. At the end of each measurement day the calibration was repeated. Fig. 5.10 shows an example calibration. Throughout the measurement the calibration drifted very slightly.

The data was recorded using a National Instruments PCI-6123 16 bit data 


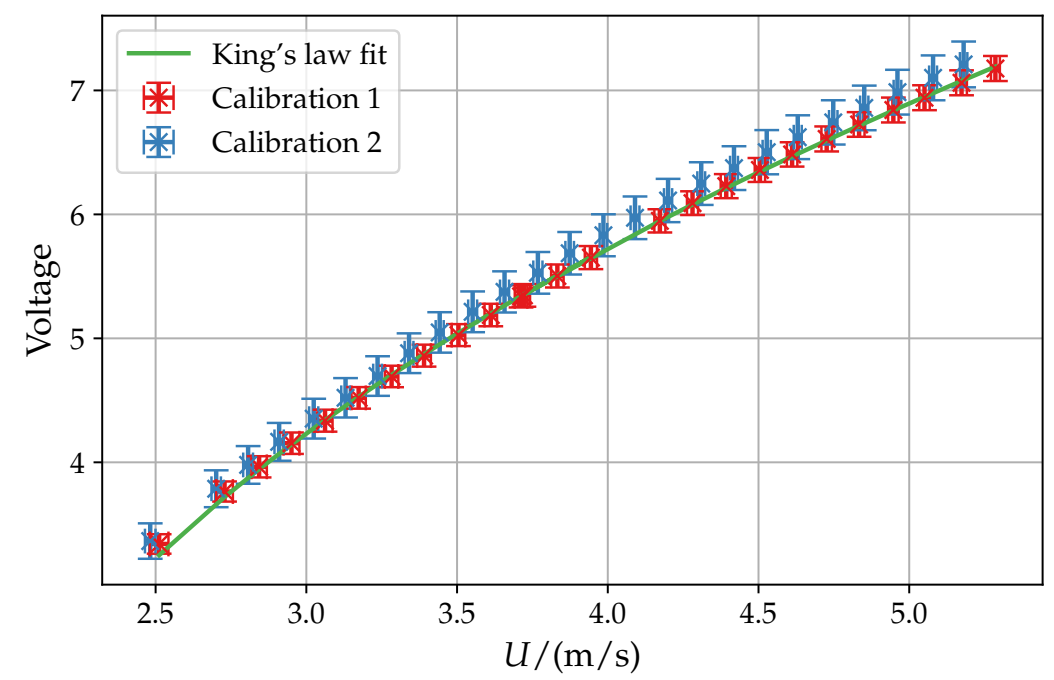

Figure 5.10: Typical calibration data with King's law fit. Calibration 1 was taken before the measurement, Calibration 2 afterwards. Errorbars indicate the standard deviation of the fluctuations around the mean while acquiring the calibration point.

acquisition card. The sampling rates were dictated by the internal low pass filters of the CTA that were set to $30 \mathrm{kHz}$ (conventional wires) or $100 \mathrm{kHz}$ (NSTAPs), such that the sampling frequencies were $60 \mathrm{kHz}$, and $200 \mathrm{kHz}$, respectively. The data was sampled in chunks of 6 Mio. samples and stored in a binary format.

During each recording the ambient pressure in the tunnel and the temperature were recorded. The fluctuations of both were typically $<2 \%$.

\subsubsection{Routine Calculations}

The same initial data analysis was applied to all hot wire datasets presented here and is described briefly in the following. Each datasets consists of multiple chunks of $N_{S}=6$ Mio velocity samples, which are analysed in parallel. The following procedures are carried out on each chunk of data. The results are averaged once all chunks have been analysed.

The voltages are converted into flow velocities using King's Law of the form $V^{2}=A+B U^{n}$ (see. Sec. 5.1). The resulting velocity arrays are flipped, such that throughout the analysis the $x$-coordinate is parallel to the flow direction.

Then, the data is filtered using an 8th-order butterworth low pass filter. The filter frequency $f_{F}$ is determined by finding the minimum of the unfiltered energy spectrum, which indicates the beginning of the frequency regime in which noise dominates. The filtering introduces edge effects, which are 
eliminated by discarding the first and last 60 values of a dataset. The energy spectrum is calculated by first calculating the fourier transform using the MATLAB implementation of FFTW routines [39]. The squared absolute value of the result is divided by $N_{s} f_{s}$, where $f_{s}$ is the sampling frequency to yield the energy spectrum.

The correlation function is calculated using MATLAB's xcorr function. This function estimates the correlation function through a fast fourier transform employing the Wiener-Khinchin-Theorem [40, 41] for speed. The velocity structure functions are calculated equally exploiting the speed of the fast fourier transform. They can be rewritten as

$$
\left\langle(u(x+r)-u(x))^{n}\right\rangle=\sum_{k=0}^{n}\left(\begin{array}{l}
n \\
k
\end{array}\right)\left\langle u(x+r)^{k}(-u(x))^{n-k}\right\rangle .
$$

This way, the fast fourier transform can be used to reduce the time required to calculate the structure functions by $>10 \times$. Further quantities routinely calculated are histograms of velocity and voltage, as well as mean velocity $\langle U\rangle$, the RMS value of the velocity fluctuations $u(t)=U(t)-\langle U\rangle$, and the mean value of squared velocity derivatives $\left\langle(\partial u / \partial x)^{2}\right\rangle$.

\subsubsection{Estimation of $\varepsilon$}

The turbulence dissipation rate

$$
\varepsilon=15 v\left\langle\left(\frac{\partial u}{\partial x}\right)^{2}\right\rangle
$$

is one of the central turbulence parameters to be estimated from a hot wire dataset. Three methods to estimate $\varepsilon$ are considered here.

Second-Order Structure Function According to Kolmogorov's $K_{41}$ theory [42]

$$
S_{2} \sim C_{K}(\varepsilon r)^{2 / 3}
$$

in the inertial range, where $C_{K}$ is the Kolmogorov constant taking values around 2.0 [31]. A measure for $\varepsilon^{2 / 3}$ is therefore the peak of $S_{2} / C_{K} r^{2 / 3}$. The advantage of this technique is that this peak lies at smaller experimental frequencies, which are less demanding for the instrumentation. The value of $C_{K}$ depends however on the Reynolds number [31]. 

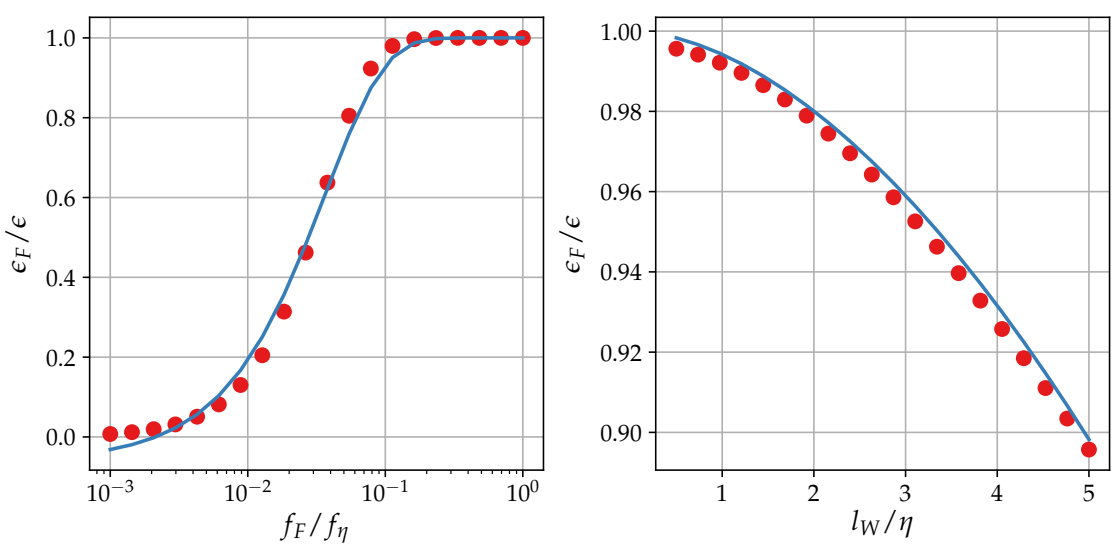

Figure 5.11: Filter Effects on the rate of dissipation estimated from a model spectrum after Pope [32]. Left: Effect of low pass filtering during post-processing. Typical value for the most demanding situations in the experiment are $f_{\eta}=U / \eta=0.1$. The blue line shows a fit of the form $1-1.06 \exp \left(-27.2 f_{F} / f_{\eta}\right)$. Right: Effect of spatial averaging across the hot wire sensor following Wyngaard [44]. Typically, $l_{W}<3 \eta$. The blue line shows $1-0.0058\left(l_{W} / \eta\right)^{1.78}$.

Third-Order Structure Function According to Kolmogorov's K41 theory [42]

$$
S_{3}=\frac{4}{5}(\varepsilon r)
$$

in the inertial range. A measure for $\varepsilon$ is therefore the peak of $S_{3} /(4 / 5) r$. As in the case of $S_{2}$, this measure of $\varepsilon$ lies in a range of frequencies that is not very demanding for the measurement system used. Further, (5.22) is an analytic result for homogeneous, isotropic, and statistically stationary turbulence at high Reynolds numbers (3.11) and no empirical constants appear. However, the approach to the $4 / 5$-law with $R_{\lambda}$ is very slow [43] for decaying flows.

Velocity Gradients The method of choice for the remainder of this part of the thesis is to directly estimate $\varepsilon$ by numerically deriving the filtered velocity fluctuation time series. This procedure is free of assumptions and valid in inhomogeneous, as well as in low- $R_{\lambda}$ flows. It is however most severely affected by finite hot wire resolution. In contrast to the effects of large-scale inhomogeneities and finite Reynolds number effects on inertial range statistics, these systematic errors introduced by spatial or temporal filtering of the small scales are well-known. It is easy to show [32] that

$$
\varepsilon=15 v \int_{0}^{\infty} k_{1}^{2} E_{11}\left(k_{1}\right) d k_{1}
$$

is identical to eq. (5.20). Therefore, a model spectrum based on a known value of $\varepsilon$ can be used to estimate the effects of different filters on the measured 
value of $\varepsilon_{m}$. Wyngaard [45] derived a formula describing the effect of spatial averaging along the length $l_{W}$ of a single hot wire on the one-dimensional spectra. This theory was applied to a model spectrum presented in Ref. [32] to estimate the effect of spatial filtering on the wire dissipation rate. The results are presented in Fig. 5.11. Equally, the effect of the employed temporal filtering to reduce noise effects can be investigated using a model spectrum. The results are shown in the left panel of Fig. 5.11. Typical values for $f_{F} / f_{\eta}$, where $f_{e} t a=U / \eta$ is the experimental frequency where $\eta$ is found, are 0.1, typical values for $l_{W} / \eta$ are $<3$. Therefore, the systematic error on $\epsilon$ measured this way is $\sim 5 \%$, which is on the order of the general experimental uncertainty for the velocity measurements. Thus, corrections for filtering effects on the dissipation rate need to be employed only when a very high accuracy is required. These results are generally confirmed by experiments investigating spatial resolution effects on turbulence statistics [46]. The correction procedure is simplified with an empirical fit to the simulated corrections to the dissipation. Such fits are added in Fig. 5.11. They are valid for single hot wires whenever the Pope model spectrum is a acceptable approximation of the energy spectrum of the flow, in particular in homogenous, isotropic turbulence. 


\section{Chapter 6}

\section{Scaling In Decaying High-Reynolds Number Turbulence}

This chapter is a reprint of the manuscript "Scaling in Decaying HighReynolds Number Turbulence", which was in the peer review process at the point of writing [1]. The author of the thesis performed the measurements, and data analysis and prepared the text. The coauthors organised the research, helped in interpreting the data, and edited the manuscript.

[1] C. Küchler, E. Bodenschatz, and G. P. Bewley. Scaling in Decaying Turbulence at High Reynolds Numbers. June 2020 


\title{
Scaling in Decaying Turbulence at High Reynolds Numbers
}

\author{
Christian Küchler* \\ Max Planck Institute for Dynamics and Self-Organization, \\ Göttingen, Germany \\ University of Göttingen, Göttingen, Germany
}

Gregory P. Bewley ${ }^{\dagger}$

Sibley School of Mechanical and Aerospace Engineering, Cornell University, Ithaca, NY, USA

\author{
Eberhard Bodenschatz $z^{\ddagger}$ \\ Max Planck Institute for Dynamics and Self-Organization, Göttingen, Germany \\ Physics Department, Cornell University, Ithaca, NY, USA \\ Institute for Dynamcis of Complex Systems, University of Göttingen, Göttingen, Germany
}

(Dated: February 20, 2021)

\begin{abstract}
We report increment statistics of turbulent velocity fluctuations and on the way they scale with increment size, which are centerpieces of turbulence theories. The data were acquired in decaying turbulence in the Max Planck Variable Density Turbulence Tunnel (VDTT). Inertial range statistics approach a universal and nontrivial shape with increasing Reynolds number. This shape is in part explained, at the larger scales, by the energy decay. We employed a model of this energydecay effect on the second-order statistics to measure corresponding scaling exponents. We found the scaling exponents to be independent of the Reynolds number and equal to $0.693 \pm 0.003$ for $2000 \lesssim R_{\lambda} \lesssim 6000$. Toward smaller scales and at high orders, the universality at high Reynolds numbers persists and does so in a way that is qualitatively similar to the larger scales and at the second order, but in a way not captured by any model, to our knowledge.
\end{abstract}

\section{INTRODUCTION}

Turbulence transfers kinetic energy from its largest scales of motion, whose dimension is $L$, to its smallest scales, measured by the Kolmogorov scale $\eta$. The energy is transferred from scale to scale at a rate $\varepsilon$, which is the power per unit mass. Turbulent fluid motion is thus multi-scale in space and time and its statistical properties are thought to be universal in the intermediate range of scales between $L$ and $\eta$, where the dynamics are dominated by fluid inertia. The extent of this intermediate range, called the inertial range, is determined by the Reynolds number of the flow, which quantifies the relative importance of inertial forces over viscous forces. The Reynolds number used here, $R_{\lambda}=u_{R M S} \lambda / \nu$, is based on the Taylor scale $\lambda$ [1]. One key question since the seminal work by Kolmogorov in 1941 [2] is the extent to which the inertial range is universal, i.e. independent of the Reynolds number and flow geometry.

In fully developed statistically isotropic turbulence of an incompressible fluid, the inertial range is characterized by scalar statistics of the velocity differences [3]. The statistics are the $n$-th order moments of the velocity increments $\Delta u(r)=u(x+r)-u(x)$, i.e., $S_{n}(r)=$ $\left\langle(\Delta u(r))^{n}\right\rangle$, and are called structure functions. At high Reynolds numbers the turbulent dissipation rate, $\varepsilon$, is the only relevant parameter in the inertial range and $S_{n}$

\footnotetext{
* christian.kuechler@ds.mpg.de

$\dagger$ gpb1@cornell.edu

‡ eberhard.bodenschatz@ds.mpg.de
}

follows scaling laws such that $S_{n} \sim r^{\zeta_{n}}$. If the statistics are self-similar with respect to scale $r$, then $\zeta_{n}=n / 3$ [2]. The observed scaling exponents, $\zeta_{n}$, deviate substantially from $n / 3$ and are a convex function of the order $n$ due to intermittency. The understanding of the role of intermittency in the energy cascade is a major problem of modern turbulence research and subject of numerous models [4-16].

Laboratory experiments [17-23], field measurements [24-28], and numerical simulations [29-31] support the existence of scaling laws, but differ in their details. In numerical simulations forced to maintain a statistical steady state and not to decay, $S_{n} \sim r^{\zeta_{n}}$ is a good approximation of the data in the inertial range $[32,33]$. Because increasing the Reynolds number is computationally costly, numerical studies have so far been limited to Reynolds numbers of 1300, and exhibit Reynolds number dependencies over the range of observed Reynolds numbers. When turbulence decays freely, power laws are more difficult to identify in computer-generated data $[34,35]$. Data from recent laboratory results of extreme statistical fidelity [36] and experiments in the atmospheric boundary layer $[28,37]$ suggest a complicated shape of $\left\langle(\Delta u(r))^{n}\right\rangle$. The latter data was acquired in the atmospheric boundary layer, whose flow parameters cannot be changed systematically. Atmospheric turbulence measurements provide important insights because the large Reynolds numbers in the atmosphere reduce the influence of large-scale effects and viscous dissipation on the inertial range. The flows are however known to be very anisotropic and inhomogeneous and the flow parameters are not controllable.

Departures from power law scaling have been sum- 
marized as finite Reynolds number effects that were expected to vanish once at large enough $R_{\lambda}$. Special data analysis schemes $[38,39]$, that have been developed to circumvent these effects, find power laws over wider ranges of scales and thus more robust estimates of $\zeta_{n}$. One established technique is to use the extended self-similar scaling that appears when plotting $S_{n}(r)$ vs $\left\langle|\Delta u|^{3}\right\rangle$ known as Extended Self-Similarity (ESS) [38]. ESS has proven to yield reproducible scaling exponents $\zeta_{n}$ across experiments pointing towards a universal regularity with respect to the order of the structure function. ESS effectively removes the parts of the structure function that do not show scaling. The underlying assumption is that the finite Reynolds number effects shadow the scaling that carries information about the general dynamics.

Despite these empirical successes and large number of studies, the shape of $S_{n}$ is still not fully explained. Numerous turbulence theories rest on the assumption that the statistics are a combination of universal scaling laws and non-universal effects that disappear at large $R_{\lambda}$.

To test this assumption, the Reynolds number must be gradually increased towards a regime, where the inertial range statistics are indeed independent of $R_{\lambda}$. The reason this has not been achieved to date, is that $L$ and $\eta$ must differ by many orders of magnitude to ensure that viscosity and flow geometry have little effect on the inertial range. This is a considerable challenge in a wellcontrolled laboratory flow. In addition, to show universality in the sense of independence from experimental parameters, the turbulence forcing and the viscosity have to be controlled independently. These properties are unified within the Variable Density Turbulence Tunnel at the MPI for Dynamics and Self-Organisation in Göttingen, Germany. The viscosity is controlled by the fluid density, while the turbulence can be forced by an extremely flexible active grid. This way, Reynolds numbers in excess of 6000 can be created while keeping anisotropy and requirements on instrumentation modest.

In this article we demonstrate how the $n$-th order structure functions approach $R_{\lambda}$-independent forms between $2000<R_{\lambda}<6000$. Increases in $R_{\lambda}$ change only the extent of the inertial range, not its shape, which is more complex than a power law. We proceed to offer a possible explanation for this shape by employing a model that accounts for the decay of turbulent kinetic energy. The functional form underlying this model allows the estimation of a scaling exponent very similar to that extracted from ESS.

\section{EXPERIMENT}

The Reynolds number of the flow in the Variable Density Turbulence Tunnel [40] (VDTT) can be finely adjusted in three largely independent ways up to levels typical for atmospheric turbulence: (i) the large-scale forcing with a novel active grid, (ii) the mean flow speed $U$ up to $5.5 \mathrm{~m} / \mathrm{s}$ by adjusting the rotation frequency of its fan, and (iii) the kinematic viscosity $\nu$ by changing the static pressure. The VDTT is filled with sulfur-hexaflouride $\left(\mathrm{SF}_{6}\right)$ at pressures 1 mbar $<p<15$ bar [40]. Flow structures of variable size are introduced using a mosaic-like arrangement of individually controllable paddles ("active grid"). It allows us to obstruct the flow on finely adjustable timeand length scales $[41,42]$. In this way we control the energy injection scale between about $0.1 \mathrm{~m} \lesssim L \lesssim 0.6 \mathrm{~m}$. The small kinematic viscosity of pressurized $\mathrm{SF}_{6}$ permits the existence of very small flow structures. The size of these structures scales with the dissipation length $\eta=\left(\nu^{3} / \varepsilon\right)^{1 / 4}$, where $\varepsilon=15 \nu\left\langle(\partial u / \partial x)^{2}\right\rangle$. For the range of ambient pressures 1 bar $<p<15$ bar, this dissipation length is between $250 \mu \mathrm{m} \gtrsim \eta \gtrsim 10 \mu \mathrm{m}$.

We record time series of hot-wire signals and convert them into one-dimensional flow fields assuming that the turbulent fluctuations are passively advedcted across the sensor by the mean flow $U$. Thus, a time step $\Delta t$ is converted to a spatial increment $\Delta x=U \Delta t$ [43]. We use a commercial constant temperature anemometer to drive and acquire data from Nanoscale Thermal Anemometry Probes (NSTAP) provided by Princeton University [44-46]. These ultra-small hot wire probes average the flow field over a length of only $30 \mu \mathrm{m}$, which is sufficient for this experiment. For flows where the turbulence length scales are larger, we also use commercial hotwires from Dantec Dynamics with sensing length $450 \mu \mathrm{m}$ $(\gtrsim 4 \eta)$. The frequencies (and wavenumbers) encountered in the measurements presented here are in a range that is not particularly demanding for this combination of sensor and anemometer circuitry [47-49]. The experiments presented here were taken under different ambient pressures and different active grid forcing schemes to allow for a careful check of the hot wire fidelity. We thus ensure the robustness of the results against probe- or flow geometry-induced biases. We emphasise that all conclusions presented here are independent of the type of probe used (NSTAP or commercial hot wire), the frequencies where turbulent fluctuations are measured, the dissipation length scale, and the active grid forcing (see Supplementary Material for more details).

\section{SMALL SCALE UNIVERSALITY}

We investigate whether the shape of the velocity increment statistics approach a universal form at large Reynolds numbers $R_{\lambda}$. In particular, we seek a universal scaling exponent $\zeta_{n}$ such that $S_{n} \sim r^{\zeta_{n}}$.

A rigorous method to find and extract power laws is to calculate the local scaling exponents

$$
\zeta_{n}(r)=\frac{d \log \left(S_{n}\right)}{d \log (r)} .
$$

$\zeta_{n}(r)$ is constant when power law scaling exists in the data. At the same time, it is a measure of the shape of $S_{n}$ free from constants that are known to be sensitive to flow configuration and Reynolds number. Fig. 1 (A) 

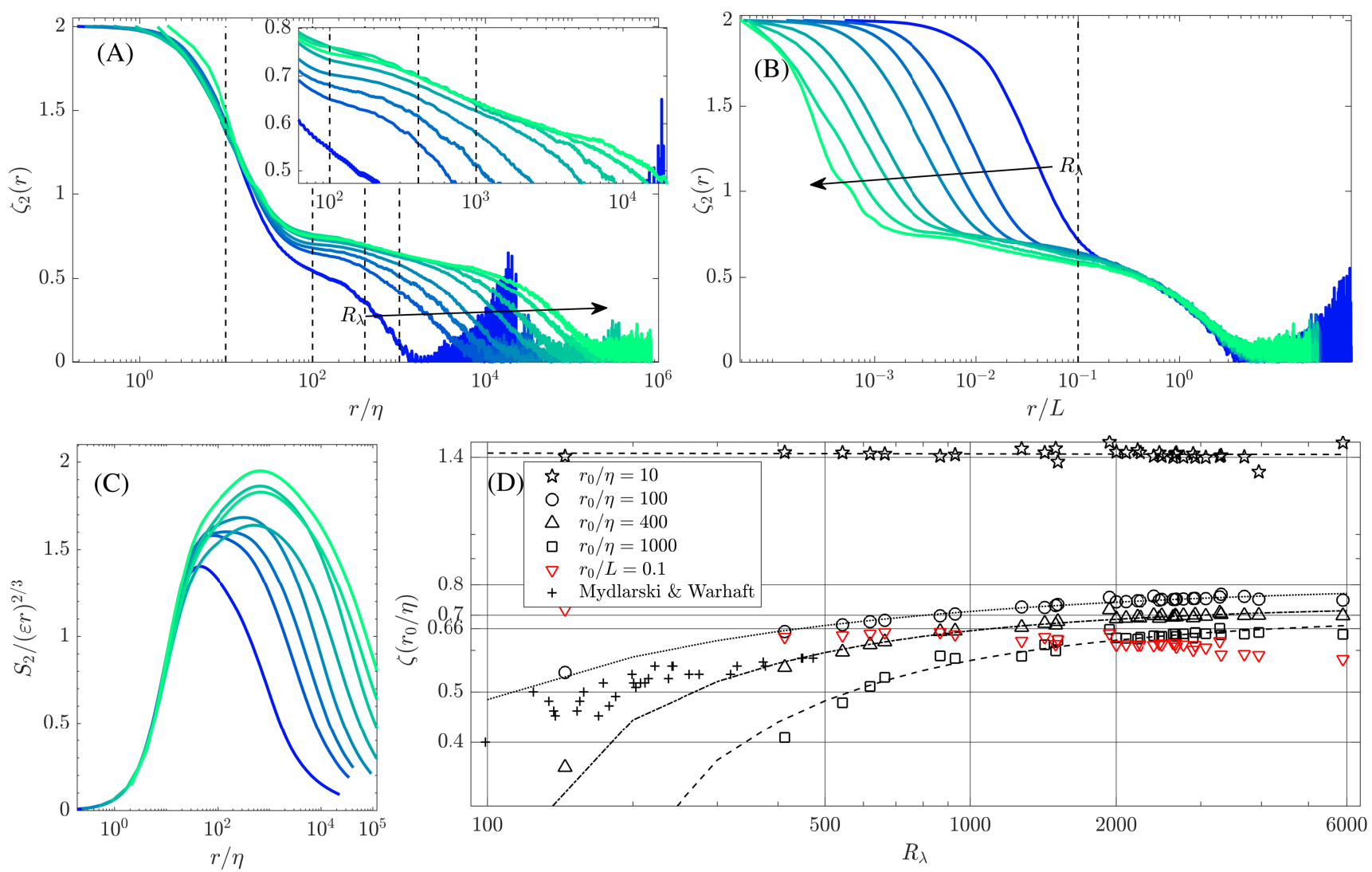

FIG. 1. (A): $\zeta_{2}(r)$ for $R_{\lambda}=144,413,620,931,1520,2400,3690$, and 5890 . The curves collapse approximately to a universal form for $R_{\lambda}>2000$ that extends from the smallest scales up to $0.1 L$ and is different from a constant, which indicates that power law scaling is masked in these data. In contrast, the curves at $R_{\lambda}<2000$ change significantly with $R_{\lambda}$. Inset: Zoom on the inertial range of the same curves. At the largest $R_{\lambda}$ a wave-like fine structure can be seen as in [36]. Dashed lines: $r_{0} / \eta$ of curves in (D); (B): Same as (A), but normalised by $L$. The curves approach a universal form from the largest scales down to $0.2 L$. (C): Structure functions $S_{2}$ compensated by the self-similar prediction $\sim(\varepsilon r)^{2 / 3}$. (D): $\zeta_{2}(r)$ evaluated at fixed $r_{0} / \eta$. The curves approach constants, but their values depend on $r$. Thus, the value of $\zeta_{2}$ (assumed constant across a wide range of scales and universal in $R_{\lambda}$ in many turbulence models) is a function of both $r$ and $R_{\lambda}$. The curves saturate at finite $R_{\lambda}$ indicating that this apparent discrepancy with the models persists as long as $R_{\lambda}$ takes finite values. The lines are fits of $C_{1}-C_{2} R_{\lambda}^{\alpha}$. The red inverted triangles correspond to the dashed line in (B), i.e. they show the scaling exponent at a fixed scale relative to $L$. Black points above this curve are within the inertial range (except for the case $r_{0} / \eta=10$ ).

shows measurements of $\zeta_{2}(r)$ for flows at different $R_{\lambda}$. Above some finite $R_{\lambda} \approx 2000$ the curves begin to collapse from the dissipation range up to $\approx 0.1 \mathrm{~L}$. The collapse in the dissipation range $r<20 \eta$ is expected and the exponent at $r \rightarrow 0$ corresponds to a Taylor expansion around that point. Around $r / \eta=100$ the curves deviate slightly from each other even for $R_{\lambda}>2000$. This region is influenced by the bottleneck effect $[36,50-53]$, whose Reynolds number dependence even at high $R_{\lambda}$ has been shown [42]. Above the bottleneck in the inertial range, the curves collapse again for $R_{\lambda}>2000$, i.e. they are are independent of $R_{\lambda}$. This is precisely the situation we looked for: The shape of $S_{n}$ does not change when increasing the separation between $L$ and $\eta$. However, the curves are not flat as would be expected from a simple power law scaling.

We investigate this observation with greater rigour in
Fig. 1 (D). We pick several values of $r_{0} / \eta$ (indicated as dotted lines in Fig. $1(\mathrm{~A})$ and $(\mathrm{B}))$ and plot $\zeta_{2}\left(r_{0} / \eta\right)$ for different $R_{\lambda}$. For each $r_{0} / \eta$, the curves approach a different constant at high $R_{\lambda}$. In contrast, if a scaling exponent were to emerge at even higher $R_{\lambda}$, these curves would approach a common constant independent of $r_{0} / \eta$ (as long as $r_{0}$ is in the inertial range). Fig. 1 (B) shows that equivalent arguments can be made when normalizing by the energy injection scale $L$ instead of the dissipation scale $\eta$.

The procedures outlined above for the case of $n=2$ are repeated in Figs. 2 and 3 for higher orders $n$ with overall similar results. The odd orders suffer from increased noise compared to even ones. In Fig. 2 all data collapse in the inertial and dissipation range with the exception of the highest $R_{\lambda}$. While this could point towards a transition at these higher $R_{\lambda}$, we regard instrumenta- 

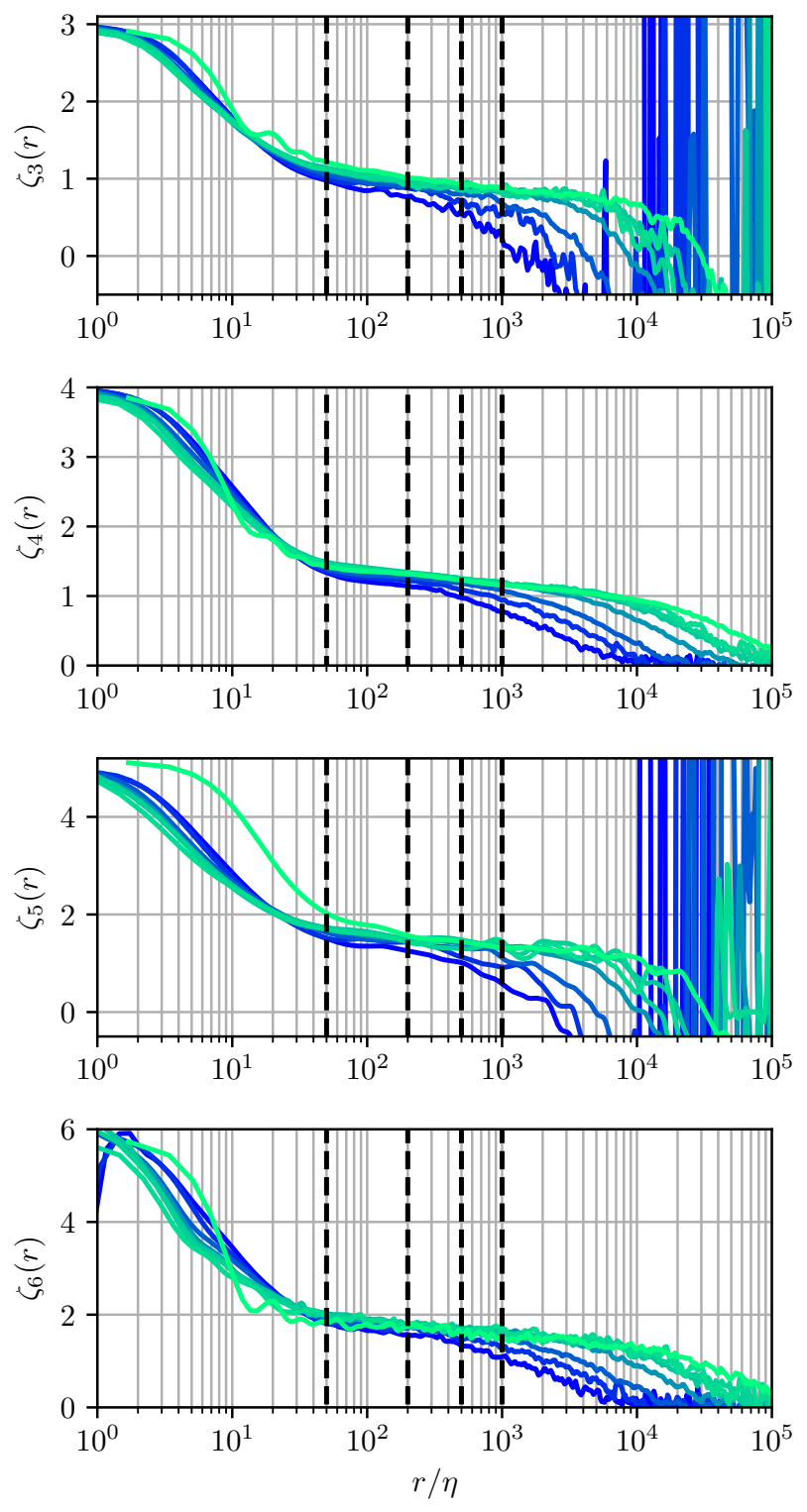

FIG. 2. Same as Fig. 1 (A), but for $2<n<7$. $\zeta_{5}$ has been smoothed using cubic splines. The curve of the highest $R_{\lambda}=5890$ is influenced by instrumentation limitations.

tion limitations as more likely. Consequently, final conclusions should not be drawn from these three datasets alone and they have been excluded when performing the fits presented in Fig. 3. As expected, $\zeta_{n}(r \rightarrow 0)=n$ for all data. Defining as the inertial range all scales between $100 \eta$ and $0.1 L$, similar inertial range trends as for $n=2$ can be identified for higher orders. With increasing $R_{\lambda}$, the local slopes become increasingly flat, i.e. $S_{n}(r)$ approaches an inertial range power law. However, this trend stops around $R_{\lambda}=2000$, even though $\zeta_{n}(r)$ still depends on $r / \eta$ in the inertial range. As for $n=2$, we have found a transition, where separating inertial, dissi-
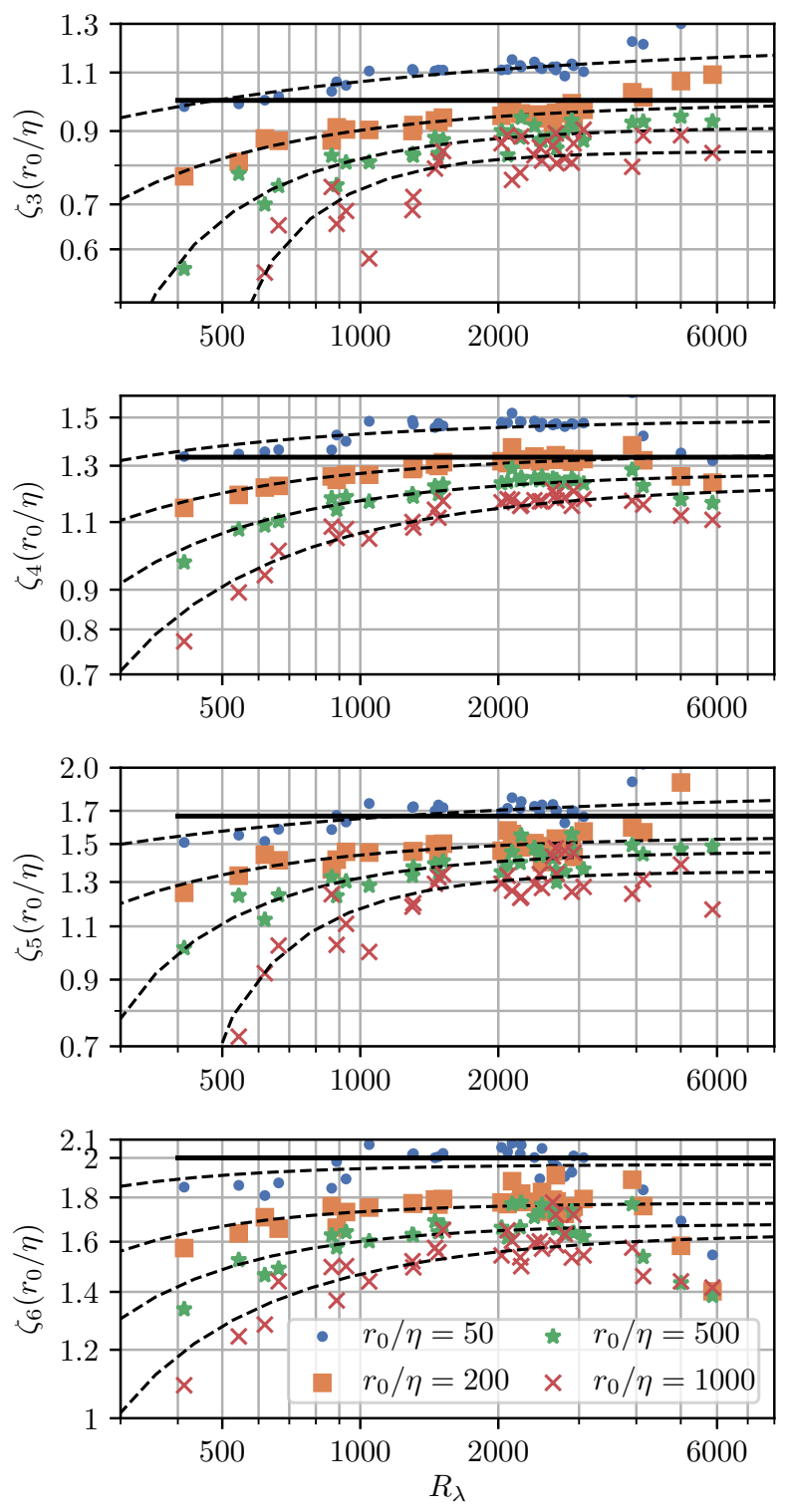

FIG. 3. Same as Fig. 1 (D), but for $2<n<7$.

pative, and energy injection scales further does not alter the shape of $S_{n}$ in the inertial range.

Similar to the case $n=2$, four different inertial range increments $r_{0} / \eta$ were selected and $\zeta_{n}\left(r_{0} / \eta\right)$ was plotted vs $R_{\lambda}$ in Fig. 3 to rigorously demonstrate this effect. For $R_{\lambda}<4000$, fits of the form $C_{1}-C_{2} R_{\lambda}^{\alpha}$ were performed. Fig. 3 shows that the asymptotic value of $C_{1}$ depends on the inertial range increment chosen at all orders studied here. 

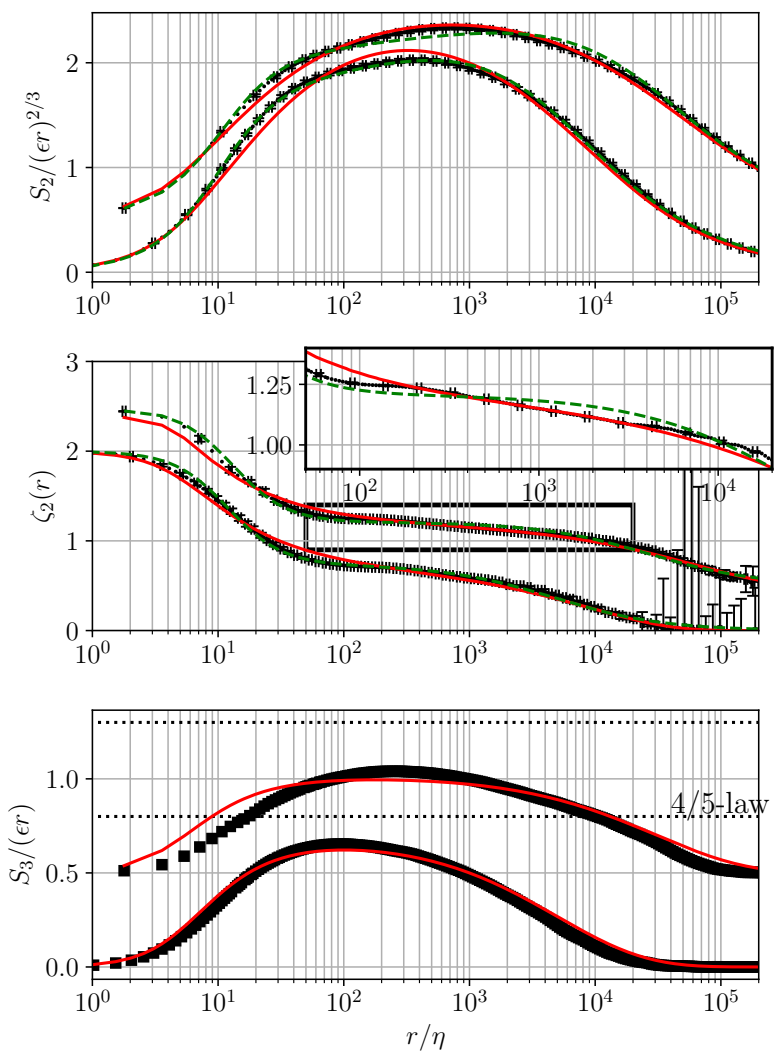

FIG. 4. Demonstration of the fit results for $R_{\lambda}=1600$ (lower curves) and $R_{\lambda}=3700$ (upper curves, offset by 0.5 for clarity). The black dots are experimental data, the red curves are the fit to the physics-based model eq. (2) with three fit parameters. The green curves represent four-parameter fits of the Batchelor interpolation formula. The region of interest is the inertial range between $100<r / \eta<10000$ in the high- $R_{\lambda}$ case as highlighted by the inset, where the red curves follow the experimental data more closely. For $r / \eta<100$ the Batchelor interpolation is superior, where the physics-based model is expected to perform poorly. At scales larger than the inertial range, both fits have a similar quality. The lower panel shows the compensated third order structure function. The high- $R_{\lambda}$ asymptote in the inertial range is indicated by dotted lines. The model prediction from the fits to $\zeta_{2}(r)$ fits the experimental data well in the low- $R_{\lambda}$ case. In the offset case of $R_{\lambda}=3700$, the model predicts an extensive scaling range that cannot be observed in the data.

\section{INTERPRETATION IN TERMS OF DECAYING TURBULENCE}

In the previous section we show that the local slopes $\zeta_{2}(r)$ of the second order structure function $S_{2}(r)$ take a universal form at finite $R_{\lambda}$ different from a clear power law scaling. These are most likely explained by departures from the ideal of homogeneous, and isotropic turbulence.

A decomposition of $S_{n}(r)$ into isotropic and anisotropic contributions might lead to much flatter $\zeta_{n}(r)$ in the isotropic sector [54]. However, such analysis requires knowledge of at least two velocity components. Instrumentation with the required spatial resolution is still in its infancy and in particular not yet compatible with large fluid densities [55].

To consider the potential effects of anisotropy, we first note that at low $R_{\lambda}<1300$ our results are in very good agreement to passive grid experiments conducted in the same facility [36], which has been shown to contain very small anisotropy [56]. $\zeta_{2}(r)$ similar to those shown in the previous section have been found in a rough-wall boundary layer, where significant large-scale anisotropy $S *=\sqrt{\nu / \varepsilon} \partial U / \partial y>0.1$ exists [57]. We have verified that the mean shear in the measurement section is small $(S *<0.02)$. Finally, our active grid dataset contains a wide variety of different wake generation schemes, which do not appear to impact our results in the inertial range. For example, $\zeta_{2}(r)$ measured behind a passive grid, and in the wake of two entirely different active grid algorithms all collapse throughout dissipation and inertial ranges. We thus argue that the observed shapes are robust against dramatic alterations in the turbulence generation mechanism and associated potential large-scale anisotropies.

The shapes found in Figs. 1 - 3 regularly appear in laboratory [36, 58] and field [28] experiments, but are in contrast to forced direct numerical integrations of the Navier-Stokes-equations[33], where $\zeta_{2}(r)$ is flatter in the inertial range. The main difference between experiments and simulations is that the latter is usually forced continuously, whereas most measurements can be performed in unsteady flow states only, where the turbulent kinetic energy decays over time. $\zeta_{2}(r)$ is tilted in simulations of decaying turbulence $[34,35]$ as it is in experiments [58]. In our experiment, the turbulent kinetic energy $u_{R M S}^{2}$ decays along the length of the measurement section, but the integral length scale $L$ remains constant or also decays over time. This is in contrast to freely decaying turbulence, where $L$ grows with time[3, 59]. We believe that the boundaries of the measurement section with cross-section $1.2 \mathrm{~m} \times 1.5 \mathrm{~m}$ (with $0.1 \mathrm{~m} \lesssim L \lesssim 0.6$ m) suppress this growth. We found this to be relatively independent of the way we estimate $L$. We chose to use $L=\int_{0}^{r_{s}}\langle u(x) u(x+r)\rangle / u_{R M S}^{2} d r$ with $\left\langle u(x) u\left(x+r_{s}\right)\right\rangle=0$. Other definitions of $L$ impact the results at small $R_{\lambda}$ and the scatter of the data otherwise.

Recent work in Yang et al. [35] provides a model spectrum based on decaying turbulence in a confined domain. It rests on an inertial range scaling $k^{-5 / 3}$ (equivalent to the self-similar scaling of $S_{2} \sim r^{2 / 3}$ ) and generic functions to describe the behaviour at the large- and dissipation scales. We have replaced the $k^{-5 / 3}$ - term by the more general $k^{-\left(\zeta_{2 F}+1\right)}$, to account for effects of potential inertial range intermittency. To extract the function that describe finite- $R_{\lambda}$ effects, the model assumes a self-similar decay of turbulent kinetic energy, i.e. $u_{R M S}(t) \sim t^{-\alpha}$, and applies a model [60] for the scale-by-scale energy 
transfer term in the evolution equation of the energy spectrum. For the case of constant $L$, the model reads (see Appendix C and [35] for details)

$E(k) \sim \frac{-A_{K}}{C}(k L)^{-\left(\zeta_{2 F}+1\right)} e^{\left(3 A_{K} / C\right)(k L)^{-2 / 3}} e^{-(1.5 / C)(k \eta)^{4 / 3}}$.

Apart from the intermittency parameter we introduced, the model contains a parameter $A_{K}$, which describes the influence of the decay. In our measurements, $A_{K}$ depends only slightly on $R_{\lambda}$. This is consistent with Fig. 1 (B) and earlier studies [59] that measured that the decay exponent and thus the large-scale part of the energy spectrum are largely independent of the Reynolds number. It further agrees with the observation that $A_{K}$ is related to the cascade efficiency $C_{\varepsilon}=\varepsilon L / u_{R M S}^{3}=\left(-A_{K}\right)^{3 / 2}$ [35, 61-63].

We fit the logarithmic derivative eq.(1) of $S_{2}$ calculated from (2) to the measured values of $\zeta_{2}(r)$. Fig. 4 compares the experimental data with the model (2) and a threeparameter Batchelor fit. The latter is a parametric fit that models the structure function shape without physical justification $[53,64]$. The Batchelor fit appears to be slightly superior in the case of low $R_{\lambda}$ (lower curves). However, as $R_{\lambda}$ increases, it cannot follow the inertial range shape. In contrast, eq. (2) does follow the experimental data in the inertial range.

The deviations around $r / \eta=60$ are due to the bottleneck effect described earlier. The model (2) allows us to obtain estimates for $\left\langle\zeta_{2 F}\right\rangle$ in the form of a fit parameter.

In the following analysis we therefore assume that a self-similar organisation of the flow structures exists, which is distorted by the nonstationarity effects induced by the decay. While the model predicts an inertial range power law to appear at very large $R_{\lambda}$, our data is in agreement with the asymptotic approach towards a complex inertial range shape different from a power law scaling.

Fig. 5 shows how $\zeta_{2 F}$ depends on $R_{\lambda}$. We observe the approach towards a common scaling exponent around $\left\langle\zeta_{2 F}\right\rangle=0.69$ for $R_{\lambda} \gtrsim 2000$. This is a little higher than the prediction for intermittency-free turbulence $\zeta_{2}=2 / 3$ and almost identical to the values that can be read off typical DNS data [30-32, 65] and the ESS estimate from the data. However, the ESS scaling exponent is practically constant around 0.69 over all $R_{\lambda}$. The range of values that $\zeta_{2}(r)$ takes between $r / \eta=100$ and $r=0.1 L$ in our measurements is indicated by the shaded region. The variation of $\zeta_{2 F}$ over one decade of $R_{\lambda}$ is much smaller than the variation in $\zeta_{2}(r)$ within a single measurement in the inertial range. We also compare the data to the measurements by Mydlarski \& Warhaft [20]. We finally revisit data that was acquired in the VDTT in the farfield of a passive grid and apply the aforementioned fit. For a passive grid the decay in this experiment has been shown by Sinhuber et al. [59] to follow the self-similar decay predicted by Saffman [66]. In this case, the integral scale grows as a function of the decay time. However, as can be seen from Fig. 3 in their paper [59], the integral length saturates at the most downstream positions. The

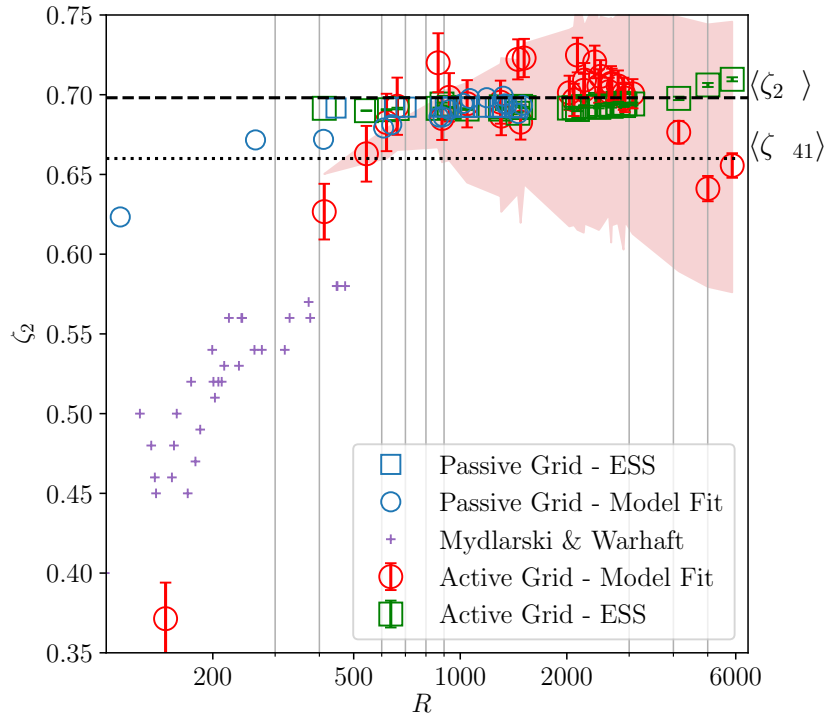

FIG. 5. Circles: Results of fitting the parameters in eq. (2). $\zeta_{2}$ approaches a constant $\left\langle\zeta_{2 F}\right\rangle=0.693 \pm 0.003$ (dashed), higher than Kolmogorov's prediction (dotted) [2]. We attribute the slight downward trend in last two data points of $\left\langle\zeta_{2 F}\right\rangle$ to the anisotropic grid forcing that was used to reach these high $R_{\lambda}$. For comparison we show the extended selfsimilarity estimates (squares), i.e. the scaling of $S_{2}\left(\left|S_{3}\right|\right)$ and the data from Mydlarski \& Warhaft [20]. The shaded region corresponds to the values that the local slope $\zeta_{2}(r)$ takes within $100 \eta<r<0.1 L$

reason is likely the same as in the active grid case investigated here: The tunnel boundaries inhibit a further growth of $L$. To make a fair comparison, we use those downstream positions in the passive grid data and apply the theory for the case of $L=$ const.

The model eq. (2) is based on a closure hypothesis that links second and third order statistics based on dimensional arguments. Thus, a prediction of $S_{3}$ can be obtained from the model (see Appendix). Fig. 4 shows that such an estimate provides good results for low $R_{\lambda}$. However, at larger $R_{\lambda}$ the model predicts a power law scaling $\sim r$, which cannot be found in the data. This is due to the aforementioned qualitative difference between the model and the data. While the model predicts the finite- $R_{\lambda}$ corrections to become less and less important, our data indicates that these effects saturate at $R_{\lambda}$ observable in the VDTT (see Fig. 1 - 3). This is more obvious at third order, where the model predicts a faster diminishing of finite- $R_{\lambda}$ effects than at second order. 


\section{DISCUSSION}

In this paper we consider the foundation of multiple models of turbulence, namely the presence of a welldefined power law scaling in the inertial range. We confirm experimental results that such a power law is a reasonable approximation to the shape of the $n$ th-order velocity increment moments $S_{n}(r)$. Existing differences between the experimental data, theoretical predictions, and numerical simulations were in the past attributed to an insufficient range of inertial scales [58, 67]. There seemed to be a consensus that in a wind tunnel at extremely large Reynolds numbers, viscosity and flow geometry would become unimportant, and $S_{n}$ would approach a clear power law of exponent $\zeta_{n}$. We test this consensus first by analysing the local scaling exponent $\zeta_{n}(r)$.

We find that the shape of $S_{n}(r)$ measured by $\zeta_{n}(r)$ follows a universal function from the dissipation scales up to the largest scales in the flow, but does not follow a power law. In particular, increasing $R_{\lambda}$ beyond 2000 does not improve the validity of $S_{n} \sim r^{\zeta_{n}}$ further. Even at $R_{\lambda} \approx 6000$ we cannot find a range of scales where $\zeta_{n}=$ const.

$\zeta_{n}(r)$ is strikingly similar throughout the inertial range for all orders $n<7$. This is in principle not surprising, since the scaling of $S_{n}\left(S_{m}\right)$ is well-documented [38, 68]. In combination with the observation of a quasi-universal inertial range, we would like to mention the functional similarity to a result based on the She-Leveque model [69], which is tightly connected to a log-Poisson distributed dissipation rate [13]. It can be recast in terms of the velocity structure function [70], which can then be decomposed into a $n$-dependent term, raised by a universal exponent $\beta$ and a term independent of the order $n$ related to the most singular structures in the flow (most likely vortex filaments).

On the grounds of the $R_{\lambda}$-independence, we infer that the physical effect that causes the observed structure function shape must (i) be active along the entire inertial range, (ii) active across different flow conditions, and (iii) similar across orders $n<7$. It is evident that we cannot make any conclusive statements about even higher $R_{\lambda}$, or even the actual limit $R_{\lambda} \rightarrow \infty$. However our data raises the question whether a power-law scaling can be observed in any terrestrial flow. It is therefore crucial to understand this shape.

In a next step, we investigate whether the decay of the turbulent kinetic energy present in the flow is a potential explanaition for the observed structure function shape at second and third order. A model of the energy spectrum derived from a popular closure model [60] while accounting for the decay [35], yields predictions of $S_{2}$ that are superior to those of a interpolation formula (Batchelor) aiming to empirically describe the effects of finite Reynolds numbers alone [64]. Our explanation is also supported by observations from numerical simulations: While $\zeta_{2}(r)=$ const. can be found in numerical simula- tions even at moderate Reynolds numbers [31], this is not possible in simulations of decaying turbulence $[34,35]$. However, the approach to the model towards an inertial range free of finite- $R_{\lambda}$ effects is much faster than supported by the data - in particular at third order.

Under the assumption that a self-similar scaling underlies the finite-Reynolds number effect, we used the aforementioned model of nonstationarity effects to extract an inertial range scaling exponent for $S_{2}$. We arrive at a value value of $\left\langle\zeta_{2 F}\right\rangle=0.698 \pm 0.011$ by averaging all values of $\zeta_{2 F}$ measured at $R_{\lambda}>2000$. This value is very close to the value $\left\langle\zeta_{E S S}\right\rangle=0.692 \pm 0.001$ extracted by comparing structure functions of different order (extended self-similarity, ESS). This suggests that the physical processes underlying the almost 30-year long successful application of ESS are small-scale universality combined with large-scale effects influencing almost all turbulence length scales.

The scaling exponent $\left\langle\zeta_{2 F}\right\rangle$ extracted from the model fit shows a clear $R_{\lambda}$-dependence, whereas the ESS estimate is constant over the whole range of $R_{\lambda}$. This study indicates that the second-order statistics scale differently at small and high $R_{\lambda}$. Nevertheless, the asymptotic scaling exponents we extract agree with the ESS estimates at much lower Reynolds numbers. It is therefore a matter of future studies to elucidate the underlying reasons. Finally, recent experimental results[36] suggest that dissipative effects occur over the entire inertial range, which is in agreement with small-scale universality from the smallest scales up to $0.1 L$ observed here.

By creating an inertial range with unmatched control over the flow parameters, we show the emergence of a $R_{\lambda^{-}}$ universal inertial range at $R_{\lambda}>2000$. We claim that dissipative and large-scale effects cause the universal inertial range to deviate from a power law scaling. We argue that knowledge of these effects allow the extraction of scaling exponents that describe the self-organisation that might be shadowed by these effects. At the same time we ask the question how relevant power laws and related concepts are practically given that they cannot be expected to dominate the statistics for at least another decade in $R_{\lambda}$.

\section{ACKNOWLEDGEMENTS}

We thank Marcus Hultmark and Yuyang Fan for providing the nanoscale hot wire probes and helping with their operation. We thank M. Sinhuber for help with using the passive grid data and helpful discussions. We thank A. Pumir, H. Xu, M. Wilczek, and D. Lohse for helpful discussions. The VDTT is maintained and operated by A. Kubitzek, A. Kopp, and A. Renner. The machine workshop led by U. Schminke and the electronic workshop led by O. Kurre built and installed the active grid. The Max Planck Society and Volkswagen Foundation provided financial support for building the VDTT. 
[1] G. I. Taylor, Statistical theory of turbulence, Proc. R. Soc. London 156A, 307 (1936).

[2] A. Kolmogorov, The Local Structure of Turbulence in Incompressible Viscous Fluid for Very Large Reynolds' Numbers, Dokl. Akad. Nauk SSSR 30, 301 (1941).

[3] P. Davidson, Turbulence - An Introduction For Scientists and Engineers (Oxford University Press, 2015).

[4] A. N. Kolmogorov, A refinement of previous hypotheses concerning the local structure of turbulence in a viscous incompressible fluid at high Reynolds number, J. Fluid Mech. 13, 82 (1962).

[5] U. Frisch, P.-L. Sulem, and M. Nelkin, A simple dynamical model of intermittent fully developed turbulence, J. Fluid Mech. 87, 719 (1978).

[6] R. Benzi, G. Paladin, G. Parisi, and A. Vulpiani, Multifractal nature of fully developed turbulence and chaotic systems., (1984).

[7] K. R. Sreenivasan and C. Meneveau, The fractal facets of turbulence, J. Fluid Mech. 173, 357 (1986).

[8] C. Meneveau and K. R. Sreenivasan, Simple multifractal cascade model for fully developed turbulence, Phys. Rev. Lett. 59, 1424 (1987).

[9] L. C. Andrews, R. L. Phillips, B. K. Shivamoggi, and J. K. Beck, A Statistical Theory for the Distribution of Energy Dissipation in Intermittent Turbulence, Phys. Fluids A 1, 999 (1989).

[10] K. R. Sreenivasan, Fractals and Multifractals in Fluid Turbulence, Annu. Rev. Fluid Mech. 23, 539 (1991).

[11] S. Kida, Log-Stable Distribution and Intermittency of Turbulence, J. Phys. Soc. Japan 10.1143/JPSJ.60.5 (1991).

[12] Z.-S. She and E. Leveque, Universal scaling laws in fully developed turbulence, Phys. Rev. Lett. 72, 336 (1994), arXiv:arXiv:1011.1669v3.

[13] B. Dubrulle, Intermittency in fully developed turbulence: Log-poisson statistics and generalized scale covariance, Phys. Rev. Lett. 10.1103/PhysRevLett.73.959 (1994).

[14] S. Grossmann and D. Lohse, Fractal-Dimension Crossovers in Turbulent Passive Scalar Signals, Europhys. Lett. 27, 347 (1994).

[15] K. R. Sreenivasan and R. A. Antonia, The Phenomenology of Small-Scale Turbulence, Annu. Rev. Fluid Mech. 29, 435 (1997).

[16] B. Mandelbrot, Scaling in financial prices: II. Multifractals and the star equation, Quant. Financ. 1, 124 (2001).

[17] S. G. Saddoughi, Veeravalli, and S. V., Local isotropy in turbulent boundary layers at high Reynolds number, J. Fluid Mech. 268, 333 (1994).

[18] H. Kahalerras, Y. Malécot, Y. Gagne, and B. Castaing, Intermittency and Reynolds number, Phys. Fluids 10 910 (1998).

[19] M. Ferchichi and S. Tavoularis, Reynolds number effects on the fine structure of uniformly sheared turbulence, Phys. Fluids 12, 2942 (2000).

[20] L. Mydlarski and Z. Warhaft, On the onset of highReynolds-number grid-generated wind tunnel turbulence, J. Fluid Mech. 320, 331 (1996).

[21] Z. She and E. Jackson, On the universal form of energy spectra in fully developed turbulence, Phys. Fluids A Fluid Dyn. 5, 1526 (1993).
[22] B. Rousset, P. Bonnay, P. Diribarne, A. Girard, J. M. Poncet, E. Herbert, J. Salort, C. Baudet, B. Castaing, L. Chevillard, F. Daviaud, B. Dubrulle, Y. Gagne, M. Gibert, B. Hebral, T. Lehner, P.-E. Roche, B. SaintMichel, and M. Bon Mardion, Superfluid high REynolds von Kármán experiment, Rev. Sci. Instrum. 85, 103908 (2014).

[23] H. L. Grant, R. W. Stewart, and A. Molliet, Turbulent Spectra From a Tidal Channel, J. Fluid Mech. 12, 241 (1962).

[24] M. M. GIBSON, Spectra of Turbulence at High Reynolds Number, Nature 195, 1281 (1962).

[25] A. Praskovsky and S. Oncley, Measurements of the Kolmogorov constant and intermittency exponent at very high Reynolds numbers, Phys. Fluids 6, 1048 (1994).

[26] K. R. Sreenivasan, An update on the energy dissipation rate in isotropic turbulence, Phys. Fluids 10, 528 (1998).

[27] G. Gulitski, M. Kholmyansky, W. Kinzelbach, B. Lüthi, A. Tsinober, and S. Yorish, Velocity and temperature derivatives in high-Reynolds-number turbulent flows in the atmospheric surface layer. Part 2. Accelerations and related matters, J. Fluid Mech. 589, 83 (2007).

[28] Y. Tsuji, Intermittency effect on energy spectrum in high-Reynolds number turbulence, Phys. Fluids 16, 11 (2004).

[29] T. Ishihara, Energy spectrum in high-resolution direct numerical, 082403, 1 (2016).

[30] T. Ishihara, T. Gotoh, and Y. Kaneda, Study of High-Reynolds Number Isotropic Turbulence by Direct Numerical Simulation, Annu. Rev. Fluid Mech. 41, 165 (2009).

[31] Y. Kaneda, T. Ishihara, M. Yokokawa, K. Itakura, and A. Uno, Energy dissipation rate and energy spectrum in high resolution direct numerical simulations of turbulence in a periodic box, Phys. Fluids $\mathbf{1 5}$ $10.1063 / 1.1539855$ (2003).

[32] N. Cao, S. Chen, and Z.-S. She, Scalings and Relative Scalings in the Navier-Stokes Turbulence, Phys. Rev. Lett. 76, 3711 (1996).

[33] K. P. Iyer, K. R. Sreenivasan, and P. K. Yeung, Reynolds number scaling of velocity increments in isotropic turbulence, Phys. Rev. E 95, 21101 (2017).

[34] D. Fukayama, T. Oyamada, T. Nakano, T. Gotoh, and K. Yamamoto, Longitudinal structure functions in decaying and forced turbulence, J. Phys. Soc. Japan 69, 701 (2000).

[35] P.-F. Yang, A. Pumir, and H. Xu, Generalized self-similar spectrum and the effect of large-scale in decaying homogeneous isotropic turbulence, New J. Phys. 20, 103035 (2018).

[36] M. Sinhuber, G. P. Bewley, and E. Bodenschatz, Dissipative Effects on Inertial-Range Statistics at High Reynolds numbers, Phys. Rev. Lett. 119, 134502 (2016).

[37] K. R. Sreenivasan and B. Dhruva, Is There Scaling in High-Reynolds-Number Turbulence?, Prog. Theor. Phys. Suppl. 130, 103 (1998).

[38] R. Benzi, S. Ciliberto, R. Tripiccione, C. Baudet, F. Massaioli, and S. Succi, Extended self-similarity in turbulent flows, Phys. Rev. E 48, R29 (1993).

[39] J. Schumacher, K. R. Sreenivasan, and V. Yakhot, Asymptotic exponents from low-Reynolds-number flows, 
New J. Phys. 9, 89 (2007), arXiv:0604072 [nlin.CD].

[40] E. Bodenschatz, G. P. Bewley, H. Nobach, M. Sinhuber, and H. Xu, Variable Density Turbulence Tunnel Facility, Rev. Sci. Instrum. 85, 1 (2014), arXiv:1401.4970.

[41] K. P. Griffin, N. J. Wei, E. Bodenschatz, and G. P. Bewley, Control of long-range correlations in turbulence, Exp. Fluids 60, 55 (2019).

[42] C. Küchler, G. Bewley, and E. Bodenschatz, Experimental Study of the Bottleneck in Fully Developed Turbulence, J. Stat. Phys. 175, 617 (2019).

[43] G. I. Taylor, The Spectrum of Turbulence, Proc. R. Soc. A Math. Phys. Eng. Sci. 164, 476 (1938).

[44] G. Kunkel, C. Arnold, and A. Smits, Development of NSTAP: nanoscale thermal anemometry probe, 36th AIAA Fluid Dyn. Conf. , 1 (2006).

[45] M. Vallikivi and A. J. Smits, Fabrication and Characterization of a Novel Nanoscale Thermal Anemometry Probe, J. Microelectromechanical Syst. 23, 899 (2014).

[46] Y. Fan, G. Arwatz, T. W. Van Buren, D. E. Hoffman, and M. Hultmark, Nanoscale sensing devices for turbulence measurements, Exp. Fluids 56, 10.1007/s00348015-2000-0 (2015).

[47] N. Hutchins, J. P. Monty, M. Hultmark, and A. J. Smits, A direct measure of the frequency response of hotwire anemometers: temporal resolution issues in wallbounded turbulence, Exp. Fluids 56, 10.1007/s00348014-1856-8 (2015).

[48] M. Samie, N. Hutchins, and I. Marusic, Revisiting end conduction effects in constant temperature hot-wire anemometry, Exp. Fluids 59, 133 (2018).

[49] A. Ashok, S. C. C. Bailey, M. Hultmark, and A. J. Smits, Hot-wire spatial resolution effects in measurements of grid-generated turbulence, Exp. Fluids 53, 1713 (2012).

[50] G. Falkovich, Bottleneck phenomenon in developed turbulence, Phys. Fluids 6, 1411 (1994).

[51] D. A. Donzis and K. R. Sreenivasan, The bottleneck effect and the Kolmogorov constant in isotropic turbulence, J. Fluid Mech. 657, 171 (2010).

[52] M. K. Verma, A. Ayyer, O. Debliquy, S. Kumar, and A. V. Chandra, Local shell-to-shell energy transfer via nonlocal interactions in fluid turbulence, Pramana 65, 297 (2005).

[53] D. Lohse and A. Muller-Groeling, Bottleneck effects in turbulence: Scaling phenomena in $\mathrm{r}$ versus $\mathrm{p}$ space, Phys. Rev. Lett. 74, 1747 (1995), arXiv:9405002 [chao-dyn].

[54] S. Kurien and K. R. Sreenivasan, Measures of Anisotropy and the Universal Properties of Turbulence, in New trends Turbul. Turbul. Nouv. Asp. (Springer Berlin Heidelberg, Berlin, Heidelberg, 2001) pp. 53-111.

[55] M. K. Fu, Y. Fan, and M. Hultmark, Design and validation of a nanoscale cross-wire probe (X-NSTAP), Exp. Fluids 60, 99 (2019).

[56] E. Bodenschatz, G. P. Bewley, H. Nobach, M. Sinhuber, and H. Xu, Variable density turbulence tunnel facility, Rev. Sci. Instrum. 85, 093908 (2014), arXiv:1401.4970.

[57] Y. Tsuji, Large-scale anisotropy effect on small-scale statistics over rough wall turbulent boundary layers, Phys. Fluids 15, 3816 (2003).

[58] R. A. Antonia, S. L. Tang, L. Djenidi, and Y. Zhou, Finite Reynolds number effect and the 4/5 law, Phys. Rev. Fluids 4, 10.1103/PhysRevFluids.4.084602 (2019).

[59] M. Sinhuber, E. Bodenschatz, and G. P. Bewley, Decay of Turbulence at High Reynolds Numbers, Phys. Rev. Lett. 114, 034501 (2015).
[60] Y.-H. Pao, Structure of Turbulent Velocity and Scalar Fields at Large Wavenumbers, Phys. Fluids 8, 1063 (1965).

[61] F. Lepreti, V. Carbone, and P. Veltri, Model for intermittency of energy dissipation in turbulent flows, Phys. Rev. E 74, 026306 (2006), arXiv:0702015 [nlin].

[62] K. R. Sreenivasan, An update on the energy dissipation rate in isotropic turbulence, Phys. Fluids 10, 528 (1998).

[63] K. R. Sreenivasan, On the scaling of the turbulence energy dissipation rate, Phys. Fluids 27, 1048 (1984).

[64] B. Dhruva, An Experimental Study of High Reynolds Number Turbulence in the Atmosphere, Ph.D. thesis, Yale University (2000).

[65] T. Gotoh, D. Fukayama, and T. Nakano, Velocity field statistics in homogeneous steady turbulence obtained using a high-resolution direct numerical simulation, Phys. Fluids 14, 1065 (2002).

[66] P. G. Saffman, The large-scale structure of homogeneous turbulence, J. Fluid Mech. 27, 581 (1967).

[67] S. Tang, R. A. Antonia, L. Djenidi, and Y. Zhou, Can small-scale turbulence approach a quasi-universal state?, Phys. Rev. Fluids 4, 10.1103/PhysRevFluids.4.024607 (2019).

[68] a. Arneodo, C. Baudet, F. Belin, R. Benzi, B. Castaing, B. Chabaud, R. Chavarria, S. Ciliberto, R. Camussi, F. Chillà, B. Dubrulle, Y. Gagne, B. Hebral, J. Herweijer, M. Marchand, J. Maurer, J. F. Muzy, A. Naert, A. Noullez, J. Peinke, F. Roux, P. Tabeling, W. V. D. Water, and $\mathrm{H}$. Willaime, Structure functions in turbulence, in various flow configurations, at Reynolds number between 30 and 5000, using extended self-similarity, Europhys. Lett. 34, 411 (2007).

[69] Z.-S. Z. She, E. Jackson, and Z.-S. Z. She, On the universal form of energy spectra in fully developed turbulence Technique for the experimental estimation of nonlinear energy transfer in fully developed turbulence On the universal form of energy spectra in fully developed turbulence, Phys. Fluids A 5, 1526 (1993).

[70] G. Ruiz Chavarria, C. Baudet, R. Benzi, and S. Ciliberto, Hierarchy of the Velocity Structure Functions in Fully Developed Turbulence, J. Phys. II 5, 485 (1995).

[71] S. B. Pope, Turbulent Flows, in Cambridge Univ. Press, Vol. 1 (2000) Chap. 6 - Scalin, pp. 232-233.

[72] A. S. Monin, A. M. Yaglom, and T. S. Lundgren, Statistical Fluid Mechanics, Vol. II, J. Appl. Mech. 43, 521 (1976).

\section{Appendix A: Measurements of Decay}

We have measured time series of velocity fluctuations along the centerline of the measurement section in various distances from the active grid with two different forcing mechanisms that produce different energy injection scales. Fig 6 shows that the turbulent kinetic energy measured by $u_{R M S}^{2}$ is decreasing at all points considered here for both grid protocols. It further shows that the integral scale is not growing, but slightly decreasing. 

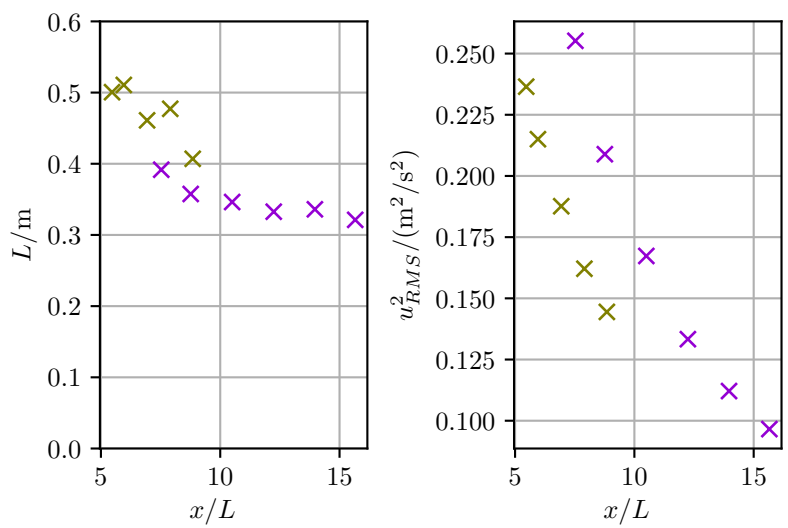

FIG. 6. Development of the integral scale (left) and turbulent kinetic energy (right) for different distances from the grid. The distances are normalised by the active grid length scale defined by the correlation lengths of the paddle protocol (see [42] for details). This scale is different from the mesh size normally used in passive grid turbulence. $L$ was estimated using from $\int_{0}^{r_{0}} C_{1} 1(r) / u_{R M S}^{2} d r$ with $r_{0}$ the first zero-crossing of the correlation function. Results for different definitions of $L$ are similar.

\section{Appendix B: Experimental Conditions}

Fig. 7 indicates critical experimental length scales along the measurements of $\zeta_{2}$. The probe averaging length mainly influences smaller scales and is far away from the region of interest. The temporal resolution is determined by the noise filtering frequency and the frequency response of the measurement system. The frequency response of the system is not perfectly flat anymore starting around $1 \mathrm{kHz}$ [47]. The range of scales we are interested in is therefore in the flat part of the frequency response curve. The noise filtering frequency is always at frequencies above $1 \mathrm{kHz}$.

\section{Appendix C: The Model Spectrum}

The evolution equation of the energy spectrum $E(k, t)$ can be derived directly from the Navier-Stokes-Equation in the isotropic case and is known as the KarmanHowarth-Lin equation.

$$
\partial_{t} E(k, t)=-\partial_{k} \Pi(k, t)-2 \nu k^{2} E(k, t) .
$$

The first term on the RHS describes the nonlinear transfer of energy from small to large wavenumbers and ultimately prevents the closure of the equation, since it is a third-order term. The Pao closure [60] used in the model by Yang et al. [35] assumes that the transfer term $\Pi$ is local in wavenumber space and has a self-similar form:

$$
\Pi(k, t)=C_{0} \varepsilon^{1 / 3} k^{5 / 3} E(k, t)
$$

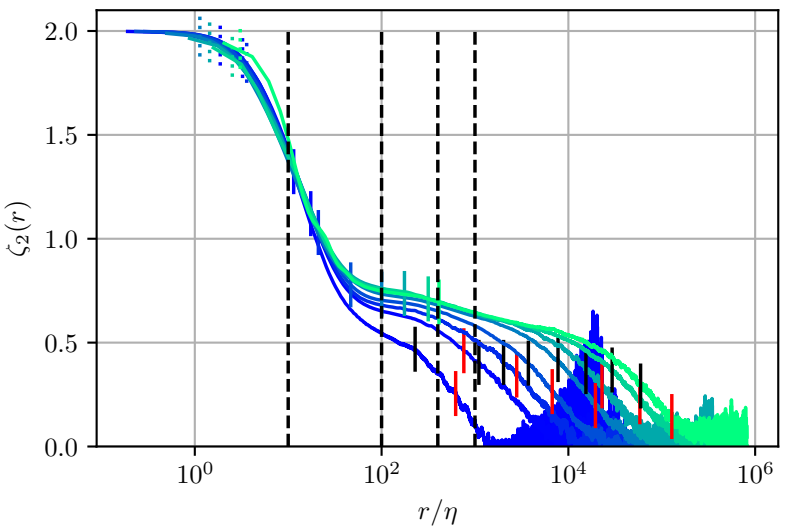

FIG. 7. Same as Fig. 1 (A) with the addition of probe length (dotted vertical lines), the value of $r / \eta$ corresponding to a measurement frequency of $1 \mathrm{kHz}$ through Taylor's Hypothesis (vertical lines), the values of $r_{0} / \eta$ chosen to assemble Fig. 1 (D) (dashed black lines), the length of the energy injection scale (vertical black lines), and the grid length scale (red lines).

The second term on the RHS of $\mathrm{C} 1$ represents the viscous dissipation at the smallest flow scales. This yields a closed form of the Karman-Howerth-Lin equation. The model by Yang et al. further assumes that the energy spectrum can be assembled by a large scale term $f_{L}(k L)$, a small scale term $f_{\eta}(k \eta)$, and a self-similar inertial range:

$$
E(k, t)=C_{k} \varepsilon^{2 / 3} k^{5 / 3} f_{\eta}(k \eta) f_{L}(k L)
$$

These assumptions are now combined with a general, selfsimilar decay of turbulent kinetic energy. In the case of a confined domain, where the parameter describing $d L / d t$ tends to zero, this model predicts the energy spectrum

$$
E(k) \sim \frac{-A_{K}}{C}(k L)^{-\left(\zeta_{2 F}+1\right)} e^{\left(3 A_{K} / C\right)(k L)^{-2 / 3}} e^{-(1.5 / C)(k \eta)^{4 / 3}} .
$$

For the purpose of measuring a scaling exponent, we replaced the term $(k L)^{-5 / 3}$ with $(k L)^{-\left(\zeta_{2 F}+1\right)}$, where the fitting parameter $\zeta_{2 F}$ is the inertial range scaling exponent for the second order structure function[71]. The parameters $C$ and $A_{K}$ are related through $C=$ $-A_{K}(6 / \pi)^{1 / 3}$. In practice, $A_{K}$ describes the large-scale part of the energy spectrum, which is heavily influenced by the decay.

The one-dimensional versions of $S_{2}$ and $E(k)$ are related through the following integral transform [72]:

$$
S_{2}(r)=\int_{0}^{\infty} E(k)\left(\frac{1}{3}+\frac{\cos (k r)}{(k r)^{2}}-\frac{\sin (k r)}{(k r)^{3}}\right) d k .
$$

To obtain the fits shown in Fig. 4, we have searched for parameters $A_{K}$, and $\zeta_{2 F}$ that yield best fits of the logarithmic derivative of eq. C5 to the experimentally measured $\zeta_{2}(r)$. 
It can be shown that $C=-A_{K}(6 / \pi)^{1 / 3}$. This quantity is related to the dissipation constant $C_{\varepsilon}=\varepsilon L / u^{3}$ relating the large scale energy injection and the small scale energy transfer rate $\varepsilon$. $A_{K}$ is the non-dimensionalized timeevolution of the energy spectrum prefactor $d\left(C_{K} \varepsilon^{2 / 3}\right) / d t$, which is a free parameter.

The energy transfer spectrum $\Pi(k)$ is related to $S_{3}$ via

$$
S_{3}=12 \int_{0}^{\infty} \frac{1}{k^{2}} \frac{\partial \Pi}{\partial k} \frac{d}{d r}\left(\frac{1}{3}+\frac{\cos (k r)}{(k r)^{2}}-\frac{\sin (k r)}{(k r)^{3}}\right) d k
$$

Therefore, the model 2 in combination with its underlying closure hypothesis C3 implicitly predicts $S_{3}$. Note that strictly speaking the combination of the intermittency-corrected model 2 and the K41-type closure (C3) yields a third order exponent $\zeta_{3}$ slightly different from 1. It is reassuring to see that instead leaving the 5/3-term in (C3) as a generic scaling and fitting the resulting model to $S_{3}$ yields $\Pi \approx$ const. in the inertial range, i.e. $S_{3} \sim r$. 


\section{Chapter 7}

\section{Extension of Eulerian Predictions to High $R_{\lambda}$}

This chapter stands in the long tradition of classical Eulerian two-point statistics. The dataset presented here allows the extension of this profound body of literature towards larger $R_{\lambda}$ than previously obtainable in laboratory experiments. It is therefore well-suited to study the approach towards the values expected in the limit of high- $R_{\lambda}$. In particular, the dissipation constant $C_{\varepsilon}=\varepsilon L / u_{R M S}^{3}$, the Kolmogorov constant $C_{K}=\max \left(S_{2} /(\varepsilon r)^{2 / 3}\right)$, the 4/5-law, and the extended self-similarity scaling are investigated.

\subsection{The Dissipation Constant}

The quantity

$$
C_{\varepsilon}=\varepsilon L / u_{R M S}^{3}
$$

was introduced in Sec 3.3. Eq. (7.1) rests on the realisation that if the viscous dissipation at small scales $\varepsilon$ is the only sink of turbulent kinetic energy and there exists an energy cascade towards these smaller scales scales, $\varepsilon$ must be at least proportional to the power injected at the largest flow scales. The power injected at scale $L$ can be estimated by dimensional arguments as $u_{R M S}^{3} / L . C_{\varepsilon}$ can therefore be seen as a cascade efficiency.

Under the hypothesis of a universal energy cascade, the cascade efficiency should be independent of the Reynolds number, i.e. $C_{\varepsilon}=$ const. for large Re. This has fundamental consequences for the inviscid limit $v \rightarrow 0$ : Since any increase in Reynolds number through a decrease in viscosity $v$ must sustain $C_{\varepsilon}=$ const. and $\varepsilon \sim v\left(\partial u_{i} / \partial x_{i}\right)^{2}$, the velocity gradients $\left(\partial u_{i} / \partial x_{i}\right)^{2}$ must diverge in this limit. The existence of singularities is an active field of math- 


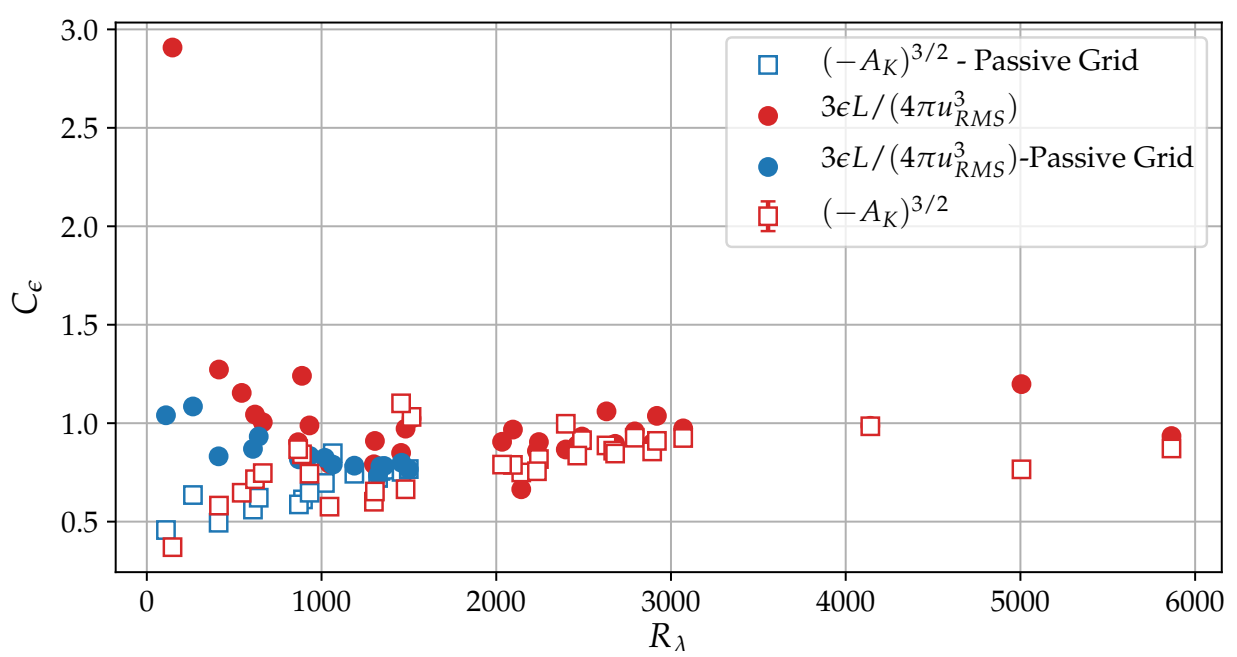

Figure 7.1: Dissipation Rate constant $C_{\varepsilon}=\varepsilon L / u_{R M S}^{3}$. Closed symbols: Data from the VDTT. Passive grid data courtesy of M. Sinhuber [50]. Open symbols denote the prediction of $C_{\varepsilon}$ from the model by Yang et al. [51] based on the fits described in Ch. 6 .

ematical research in both the inviscid Euler equations, and the Navier-Stokes equations $[48,49] . \varepsilon L / u_{R M S}^{3}=$ const can be reformulated in terms of the enstrophy $\left\langle\omega^{2}\right\rangle=\varepsilon / \nu$ (see eq. (2.21)). Inserting this into eq. (7.1) yields

$$
C_{\varepsilon}=\left\langle\omega^{2}\right\rangle \nu L / u_{R M S}^{3}
$$

Therefore, a reduction in $v$ in an otherwise unchanged flow must be accompanied by an increase in the enstrophy. In combination with the blow-up of gradients, the constancy of $C_{\varepsilon}$ points towards the regular appearance of small, structures of intense vorticity, i.e. vortex filaments.

The measurement of $C_{\varepsilon}$ poses two difficulties. First, $\varepsilon$ is notoriously difficult to estimate from hot-wire data as discussed in Sec. 5.3.3. Here, $\varepsilon=$ $15 v\left(\partial u_{1} / \partial x_{1}\right)^{2}$ was chosen and no corrections were applied. The second complication is the measurement of $L$. The most common way to estimate $L$ is the integral over some part of the autocorrelation function of the velocity fluctuations.

$$
L_{u}=\int_{0}^{r_{0}}\langle u(x+r) u(x)\rangle d r
$$

The result depends sensitively on the choice of $r_{0}$. At large $r, C_{11}=\left\langle u_{1}\left(x_{1}+\right.\right.$ $\left.r) u\left(x_{1}\right)\right\rangle$ fluctuates around 0 . While the theoretical limit of $r_{0} \rightarrow \infty$ cannot be realised in any real measurement, the location of the first zero-crossing of $C_{11}$ is often used for $r_{0}$ instead. This choice has been employed here as well. In active grid turbulence, $C_{11}$ can have nonzero values even for very large $r[52,53]$. This has motivated alternative integration boundaries, since it is 
unclear whether the long-range correlations present at these very large separations significantly contribute to the nonlinear cascade. Pearson et al. [54] argued that estimating the energy injection scale from the peak of the spectral quantity $k_{1} E_{11}\left(k_{1}\right)$ provides a more universal estimate of $L$ and improves the collapse of data from different types of flow.

Fig. 7.1 shows the values of $C_{\varepsilon}$ extracted from the hot-wire data. A comparison is made to data acquired by M. Sinhuber [50]. Further, the model of decaying turbulence in a confined domain introduced in Ch. 6 connects $C_{\varepsilon}$ to the decay parameter $A_{K}=-C_{\varepsilon}^{2 / 3}$. The value of $C_{\varepsilon}$ corresponding to the fitted values of $A_{K}$ is shown by open symbols for both the active and the passive grid cases. The data shows a good collapse at $R_{\lambda}>500$ towards a value of slightly below 1 . No $R_{\lambda}$-dependence can be discerned up to $R_{\lambda} \approx 6000$ confirming the prediction of $C_{\varepsilon}=$ const. at large Reynolds numbers. To the author's knowledge this is the largest $R_{\lambda}$, where this was measured in a classical fluid with negligible mean shear. Risius et al. [55] investigated $C_{\varepsilon}$ under atmospheric conditions on a mountain research station finding $C_{\varepsilon} \approx 0.5$ over a comparable range of $R_{\lambda}$. Eq. (7.2) was explored in the SHREK experiment (Superfluid Helium high REynolds number von Kármán flow) up to $R_{\lambda} \approx 30000$ in superfluid helium [56] showing that both viscous and superfluid helium approach a constant at large $R_{\lambda}$. At lower $R_{\lambda}$, an abundance of measurements of $C_{\varepsilon}$ exists $[57,58,59,60,54,61,53]$. The high- $R_{\lambda}$ asymptotic value appears to depend on the turbulence generation mechanism and typically varies between 0.5 and 1 . Forced DNS and [62] active and fractal grid turbulence $[63,64]$ appear to result in values close to 1 , whereas passive grid data and sheared flows result in lower values around 0.5 [6o].

\subsection{The Kolmogorov Constant and the 4/5-th Law}

This section offers results from the VDTT on two classical inertial range predictions at large Reynolds number. By averaging the Navier-Stokes equations, one obtains the exact Karman-Howarth relations (see Sec. 3.2). In terms of the structure functions it reads for the isotropic case [67]

$$
-\frac{2}{3} r^{4} \varepsilon-\frac{r^{4}}{2} \frac{\partial S_{2}}{\partial t}=\frac{\partial}{\partial r} \frac{r^{4} S_{3}}{6}-v \frac{\partial}{\partial r} \frac{r^{4} \partial S_{2}}{\partial r} .
$$

Under the assumption of statistical stationarity, the first term on the LHS vanishes. In the limit of large Reynolds number, the last term on the RHS becomes negligible for $r \gg \eta$. Upon integration, one therefore recovers the 

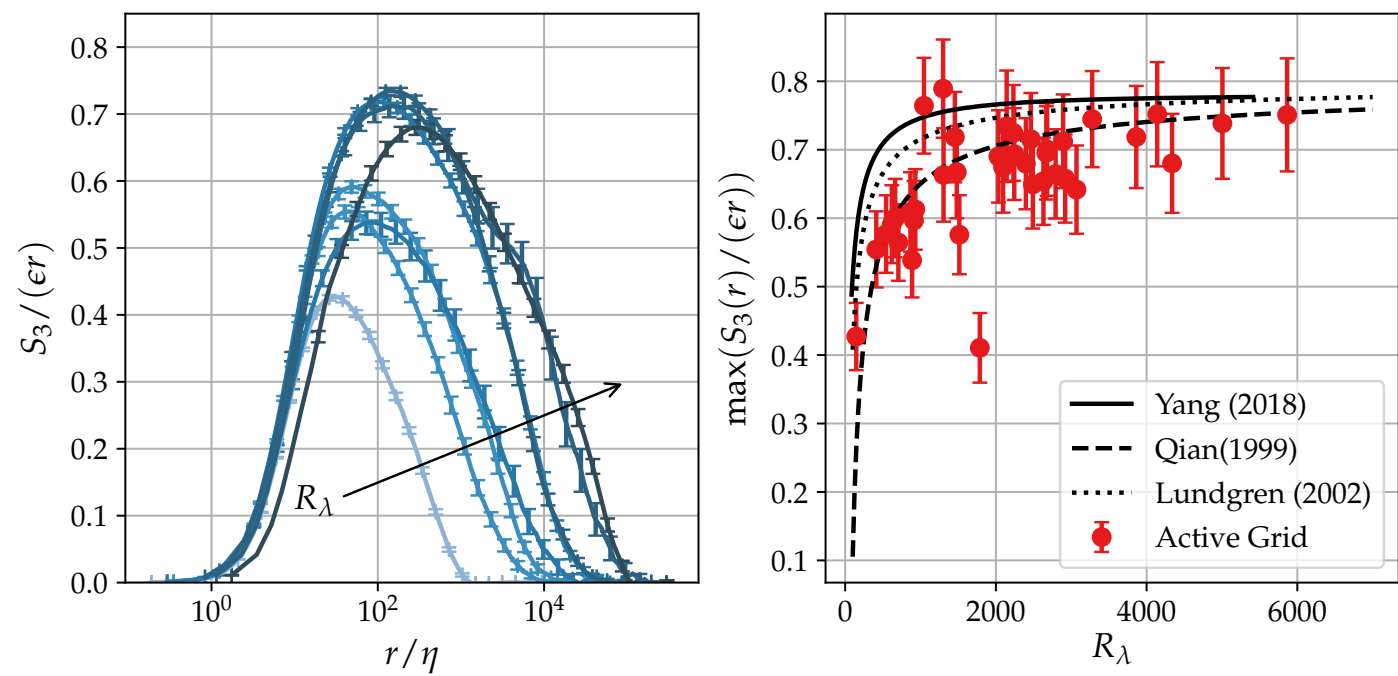

Figure 7.2: Left: $S_{3} /(\varepsilon r)$ as function of scale $r / \eta$ for selected $R_{\lambda}=147,413,619,888,1550$, 2143,2892 , and 5865 . Errors include $95 \%$ CI of the statistical error, a $3 \%$ error from velocity calibration, and a $5 \%$ error on the dissipation measurement. The incomplete collapse at the highest $R_{\lambda}$ is most likely due to insufficient small-scale resolution. Note a bump in the largescale cutoff. Right: Value of the peak of $S_{3} /(\varepsilon r)$ as function of $R_{\lambda}$ along with models by Qian [65], Lundgren [66], and the Yang model dicussed in detail in Ch. 6.

4/5th-law

$$
S_{3}(r)=\frac{4}{5} \varepsilon r
$$

in the inertial range for large enough $R_{\lambda}$. Since this relation can be derived in an exact way for homogeneous, isotropic turbulence, it must be approached in the limit of extremely large $R_{\lambda}$. It is however observed, that in decaying turbulence such as in the wake of an (active) grid, the compensated thirdorder structure function $S_{3} /(\varepsilon r)$ approaches o.8 rather slowly [68]. This has been related to instationarity effects, i.e. the second term on the RHS of eq. (7.4) does not vanish. In numerical simulations of turbulence in a forced periodic box, nonstationarity effects decay quickly, and only viscous effects reduce $S_{3} /(\varepsilon r)$ in the inertial range. Consequently, eq. (7.5) is fulfilled at lower $R_{\lambda}$. To the author's knowledge, the data obtained in the ONERA wind tunnel in Modane at $R_{\lambda}=2260$ constitute the highest $R_{\lambda}$ at which the $4 / 5$ law was studied experimentally $[69,68]$ until now. This thesis extends this range towards $R_{\lambda} \approx 6000$ as shown in Fig. 7.2.

As discussed in $\mathrm{Ch}$. 6 , the scaling range $S_{3} /(\varepsilon r)=$ const. cannot be identified due to effects of finite Reynolds number, and lack of statistical stationarity. The approach to the 4/5-law is studied nevertheless by observing the increase of the maximum of the compensated structure function for different $R_{\lambda}$. The active grid data approaches 0.8 slightly slower than the predictions by 

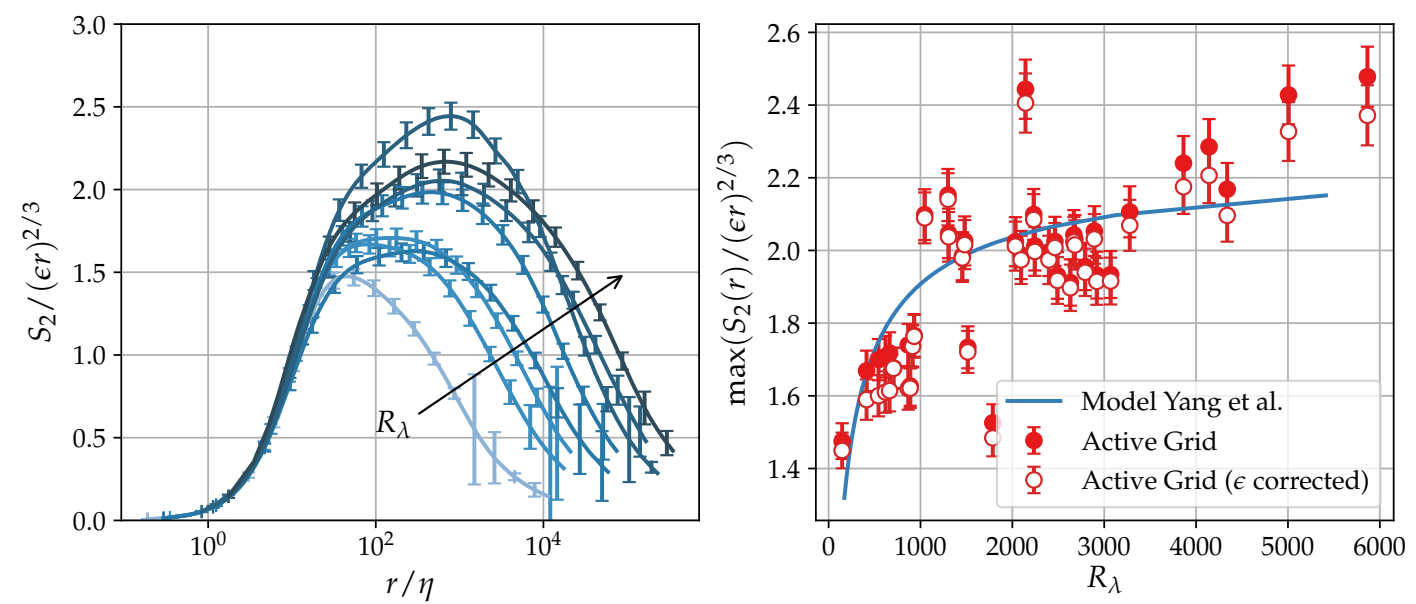

Figure 7.3: Left: $S_{2} /(\varepsilon r)^{2 / 3}$ as function of scale $r / \eta$ for selected $R_{\lambda}=147,413,619,888,1550$, 2143, 2892, and 5865. Errors as in Fig. 7.2. Right: Value of the Kolmogorov constant $C_{K}$ as function of $R_{\lambda}$ along with the prediction by the Yang model [51] dicussed in detail in Ch. 6 .

Yang et al. [51] and Lundgren [66], which account for finite-Reynolds number effects and decaying turbulence using matched asymptotic expansions. The data is well-described by the one-parameter fit to $0.8-C R_{\lambda}^{-2 / 3}$ predicted by Qian for decaying turbulence [65].

Combining equation (7.4) with the $\mathrm{K}_{41}$ framework reveals that the skewness $S=S_{3} / S_{2}^{3 / 2}$ is related to the Kolmogorov constant $C_{K}[67]$ in the inertial range:

$$
S_{3} / S_{2}^{3 / 2}=-\frac{4}{5} C_{K}^{-3 / 2}
$$

$C_{K}$ is related to both the prefactor of the energy spectrum and $S_{2}$ and shall be defined here as

$$
C_{K}=\max \left(\frac{S_{2}}{(\varepsilon r)^{2 / 3}}\right) .
$$

A consequence of the Kolmogorov framework is that $C_{K}$ is a universal function, i.e. independent of the flow under study. In particular, it should be independent of $R_{\lambda}$. Experiments and simulations regularly find values around 2.0, albeit with more scatter from experiment to experiment than for the $4 / 5$ thlaw [70, 71, 72]. Fig. $7 \cdot 3$ shows the Kolmogorov constant as a function of $R_{\lambda}$.

Unlike the corresponding quantity for $S_{3}$, no clear approach towards a high- $R_{\lambda}$ asymptote can be identified even after correction of probe induced biases into $\varepsilon$ in the spirit of Sec. 5.3.3 (open symbols). However, the measurement of $\varepsilon$ relies on local isotropy. In weakly anisotropic flows, $\varepsilon$ is always higher than estimated by the isotropic estimate [71]. Similarly, the scatter is relatively large when compared to $S_{3} /(\varepsilon r)$. This is particularly interesting, 

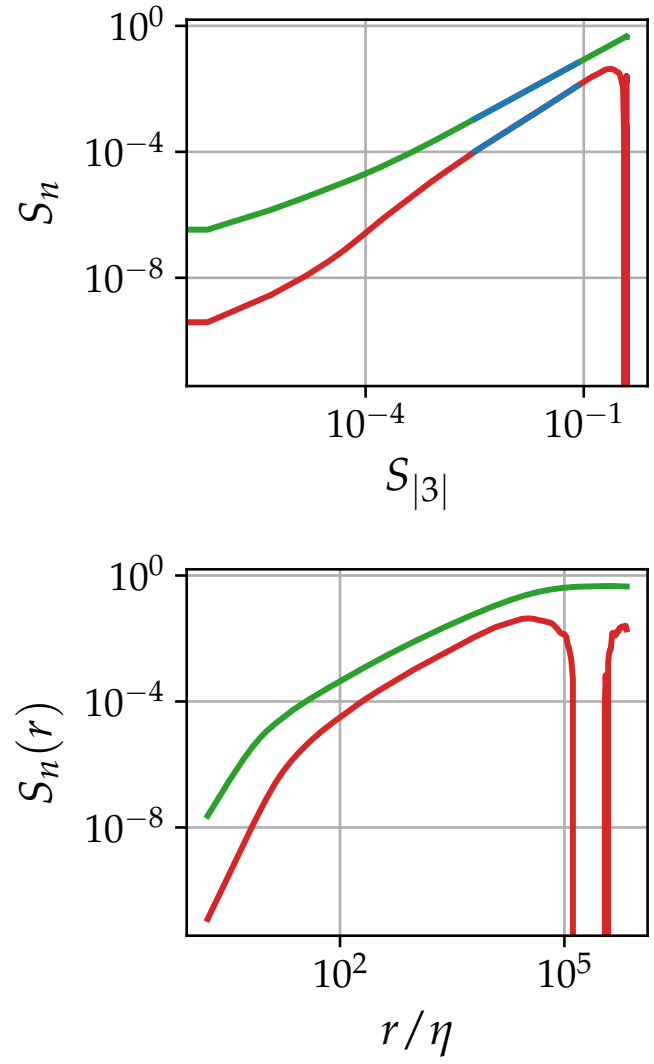

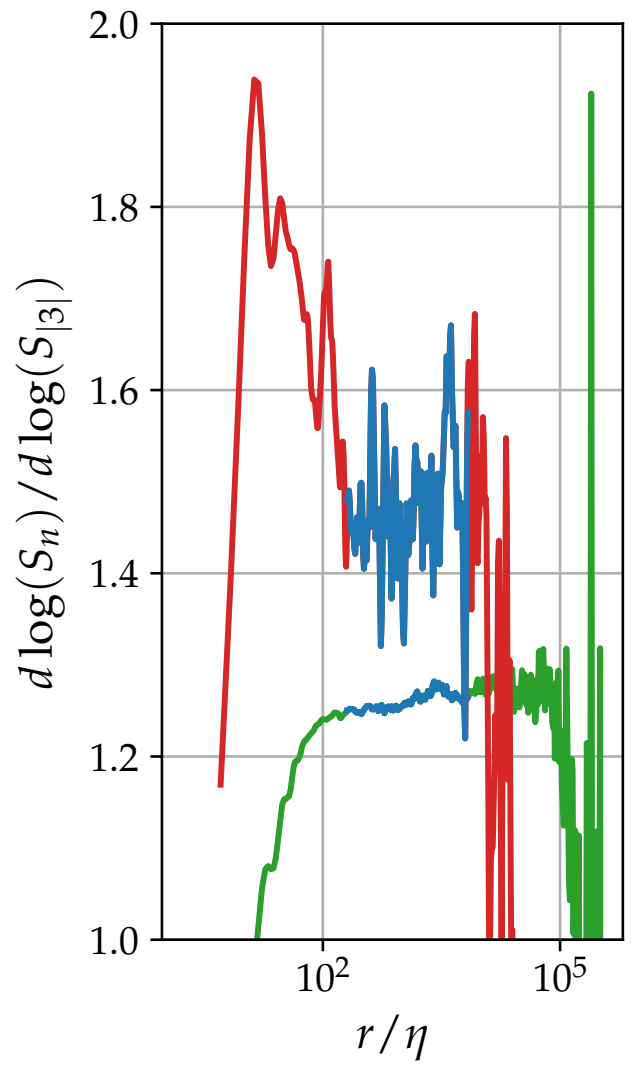

Figure 7.4: Illustration of ESS scaling for $n=4$ (green) and $n=5$ (red) for $R_{\lambda}=4141$. Left: Illustrative example that $S_{n}$ vs $S_{|3|}$ scales over a wider range of scales than $S_{n}$ vs. $r$. Right: Procedure used to extract ESS scaling exponents. The blue range indicates the averaging area interpreted as inertial range.

because the shape of $S_{2}$ and $S_{3}$ when measured by the logarithmic derivative is both independent of $R_{\lambda}$ and shows only little scatter from experiment to experiment (see Ch. 6).

\subsection{ESS-Exponents at large $R_{\lambda}$}

An influential result regarding scaling of the structure functions $S_{n}$ is that in experimental and numerical data, $S_{n}$ scales with $S_{m}$ considerably better than with $r$ [73, 74]. This procedure is known as extended self-similarity (ESS). In particular, the inertial range scaling is extended towards large scales, if $S_{n}\left(S_{|m|}\right)$ is plotted, where $S_{|m|}(r)=\left\langle|u(x+r)-u(x)|^{m}\right\rangle$. It is convenient to choose $m=3$, since $S_{3} \sim r^{1}$, such that the ESS scaling exponent is directly related to the actual scaling exponent $\zeta_{n}$. Grossmann et al. [75] found that the usage of the absolute velocity difference is essential in the application of ESS. 
They suggest in fact that the scaling $S_{|n|}\left(S_{|m|}\right)$ shows an even better scaling. This is, because the ESS procedure extends the inertial range scaling primarily towards large scales, where the scaling behaviour differs drastically between even and odd order structure functions. The scaling of the absolute velocity differences is $r^{0}$ for large $r$, whereas the scaling of the pure velocity differences is $r^{0}$ for even orders only. In all cases, $S_{n}(r<\eta) \sim r^{n}$ at small scales, such that an extended scaling cannot be expected here.

ESS became a standard tool to extract scaling exponents in flows where the inertial scaling range is nonexistent or small due to insufficient $R_{\lambda}$, or strong large-scale inhomogeneities. In this section the scaling exponents are extracted by calculating

$$
\zeta_{n}^{\mathrm{ESS}}(r)=\frac{d \log \left(S_{n}\right)}{d \log \left(S_{|3|}\right)}
$$

The ESS scaling exponent $\zeta_{n}^{\mathrm{ESS}}$ is then taken as the mean of $\zeta_{n}^{\mathrm{ESS}}(200 \eta<$ $r<0.2 L$ ), which is indicated by blue overlays in Fig. 7.4. The procedure is illustrated in Fig. $7 \cdot 4$ for $n=4$ and 5. It illustrates that the scaling range for even orders is extended, whereas the inertial range for odd orders is about as large as in the direct scaling $S_{n}(r)$. Nevertheless, the local slope of $S_{n}\left(S_{|m|}\right)$ is substantially flatter than $d \log \left(S_{n}\right) / d \log (r)$ (see Fig. 8.1). It was shown in Ch. 6 that the ESS estimate of $\zeta_{n}$ compares well to alternative methods of investigating the scaling exponent at second order.

The scaling exponents extracted in this fashion are illustrated in Fig. 7.6 for three $R_{\lambda}(1305,3070,4141)$. For $n<9$, no Reynolds number dependence of $\zeta_{n}^{\mathrm{ESS}}$ can be identified. At $n>9$, the data is not statistically converged as indicated in Fig. 7.5 and longer datasets would have to be taken to reduce the statistical uncertainty. For completeness, the unconverged exponents are shown as open symbols in the inset of Fig. 7.6.

The data is compared to several models of $\zeta_{n}$. The Kolmogorov prediction from 1941 [42] without inertial range intermittency states that $\zeta_{n}=n / 3$. This is clearly violated even at the largest $R_{\lambda}$ indicating the presence of inertial range intermittency. Furthermore, no trend can be observed that would indicate a slow approach towards $\zeta_{n}=n / 3$ at any terrestrial $R_{\lambda}$. The refined similarity hypothesis [76] accounts for a intermittent distribution of $\varepsilon$ in space. It assumes in particular that $\varepsilon$ is log-normally distributed and the $n$-th order moments of the velocity increment statistics therefore scale with $(n / 3)-\mu / 18(n(n-3))$, where $\mu$ is a free intermittency parameter $\approx 0.2$. Here, $\mu$ was taken as a fit parameter to the highest $R_{\lambda}$ data. The fit yields 


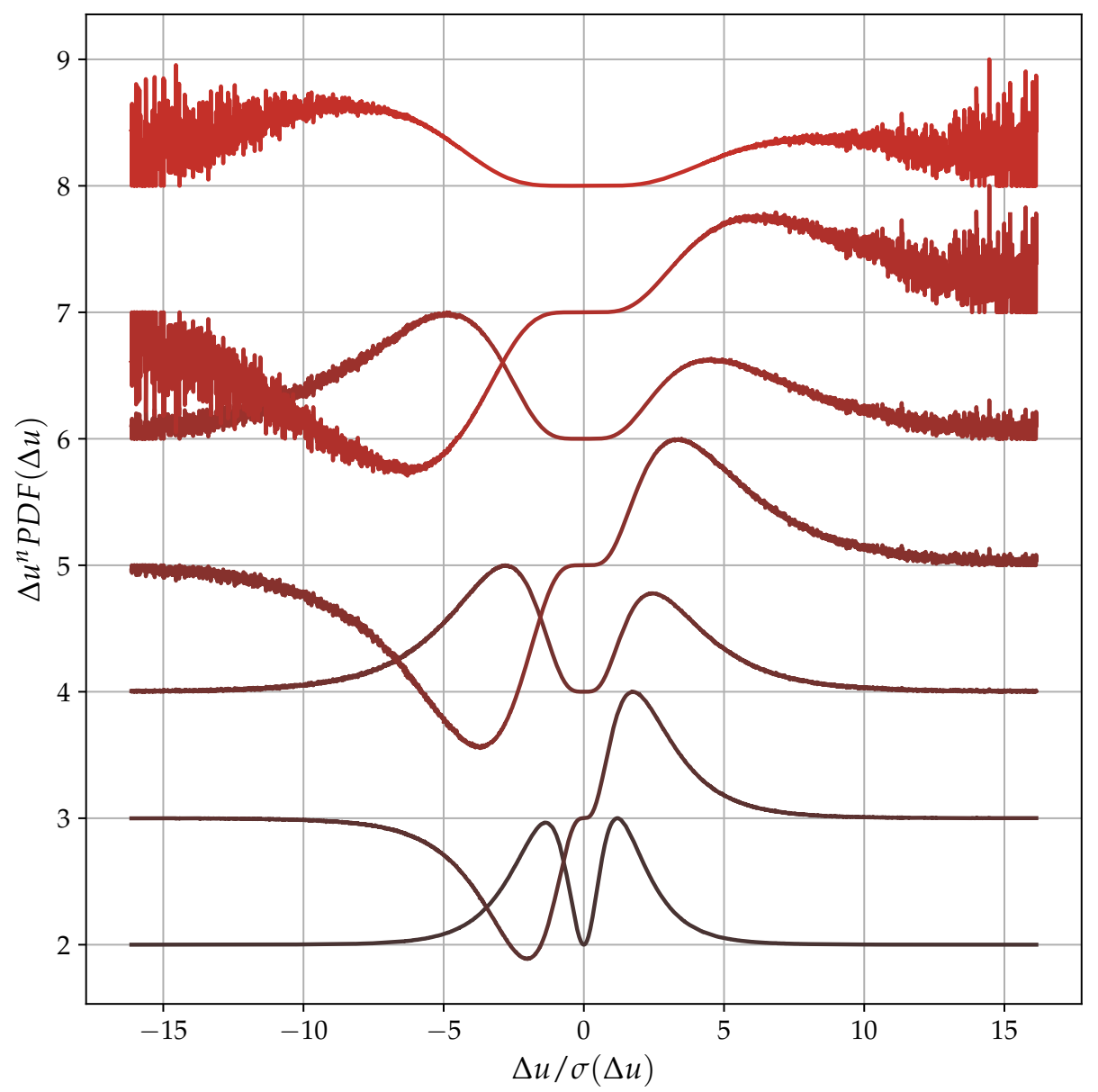

Figure 7.5: $n$-th order moment PDF for $2<n<8$ incrementally offset by 1 for clarity. The increments were taken over $r=242 \eta$. The PDFs return to o for $n<9$ indicating statistical convergence. 


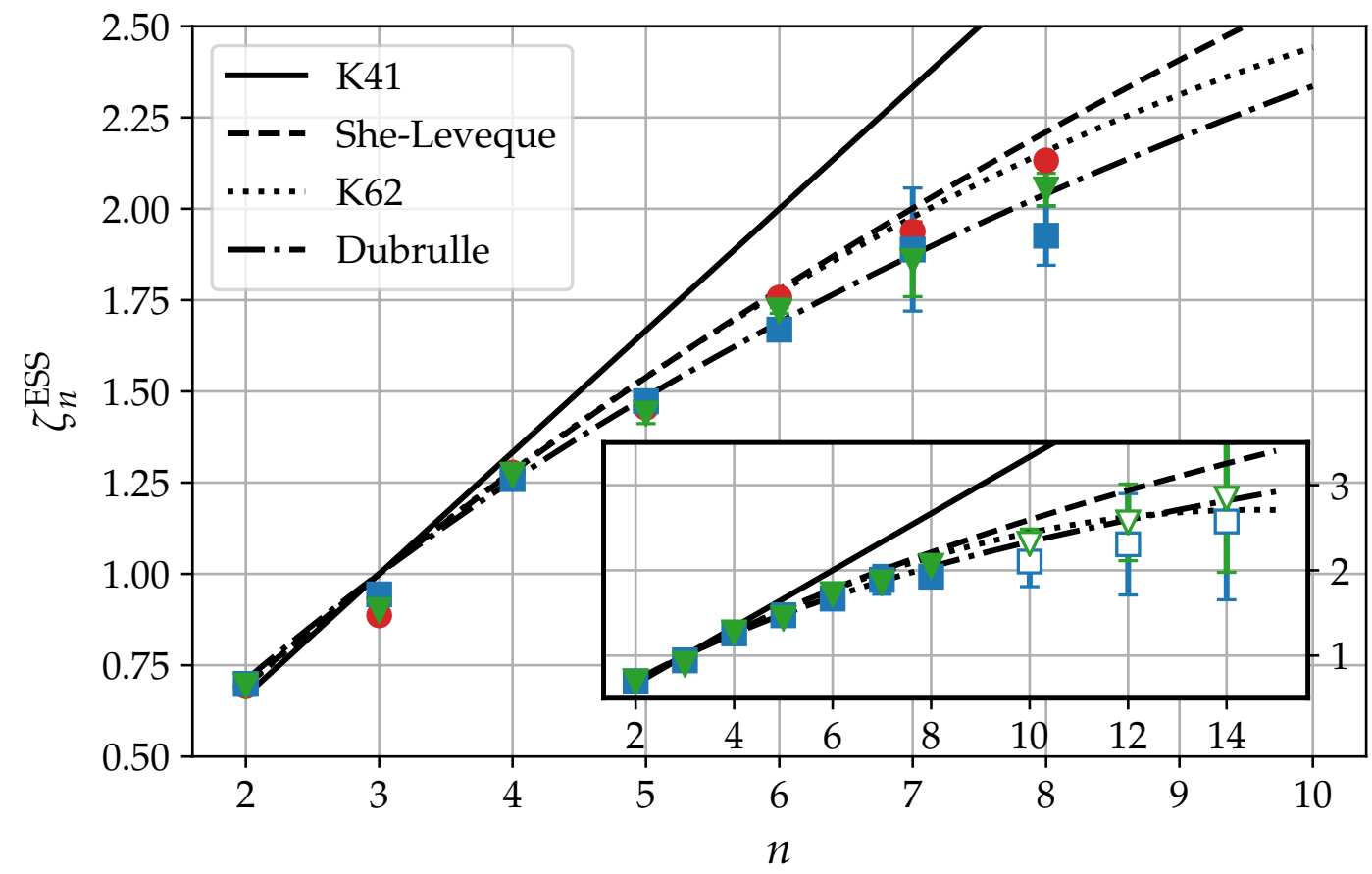

Figure 7.6: ESS exponents as function of order $n$. Three datasets were chosen: $R_{\lambda}=4141$ (blue squares), $R_{\lambda}=3070$ (green triangles), and $R_{\lambda} \approx 1305$ (red circles). Inset: Extension towards higher orders. The data for $n>8$ is not statistically converged and consequently shown in open symbols.

$\mu=0.23 \pm 0.01$, which is in good agreement with previous measurements in the atmosphere $[77,72]$. The She-Leveque model assumes that the moments of the dissipations form a hierarchy. This hierarchy of moments approaches a constant in the limit of $n \rightarrow \infty$, which corresponds to the dissipation of the vortex filaments. This model predicts $\zeta_{n}=n-9+2\left(1-(2 / 3)^{n}\right)$. It overestimates the scaling exponents, i.e. predicts weaker intermittency levels than the data indicates, but only slightly so. Dubrulle [78] showed that the She-Leveque model is a special case of Log-Poisson distributed dissipation statistics, which she generalised (see Sec. 3.3.4 for details). The most dissipative structures that dominate the statistics of $n \rightarrow \infty$ are allowed to have any codimension, whereas the She-Leveque model requires them to be onedimensional, i.e. to be vortex filaments. The Dubrulle model further contains a parameter for the intermittency level $\beta$. Unsurprisingly, the two-parameter fit describes the data better than the She-Leveque model.

In summary, this section confirms previous findings that significant departures from the 1941 Kolmogorov prediction exist and that they become more pronounced at higher orders. This is commonly attributed to the intermittent nature of dissipative structures. After taking into account the statistical 
uncertainty, the data presented here show no significant $R_{\lambda}$-dependence, in accordance with the findings in Ch. 6 and results from measurements with a passive grid installed in the VDTT [50]. The data further confirms the findings in Ch. 6 that the measurement of a scaling exponent in structure functions is a delicate task bearing substantial uncertainties. The data does not clearly favor one of the models shown in Fig. 7.6 and cannot lend particular support to any of the associated theories. 


\section{Chapter 8}

\section{Log-Periodic Oscillations in Even-Order Structure Functions}

\subsection{Introduction}

The data presented in this thesis is uniquely suited to provide insights into the inertial range scaling, because it permits the study of scale-dependent statistics, such as the longitudinal structure function $S_{2}$ with unprecedented detail. In particular, statistical uncertainties can be mitigated to a large degree, because the sampling of thousands of large eddies is very cheap compared to to high-resolution DNS, whose results are oftentimes averaged over only a couple of large eddies or even less than one [79, 80]. Compared to previous wind tunnel experiments, the data presented here allow an examination of the inertial range at scales, where both the scale of turbulence forcing and viscous dissipation are so far apart that their continued separation does not appear to significantly change the inertial range shape. Features found in the inertial range can therefore be reliably distinguished from a "bleed-in" of either the energy-injection or the dissipation scale.

This chapter investigates the scale-by-scale behaviour in the inertial range in detail. We are in particular interested a fine-structure, which has been found in wind tunnel data of extreme statistical fidelity [81], but whose extent is still a matter of debate [82]. This fine-structure is reminiscent of the prediction of log-periodic oscillations and lacunarity, which have been speculated to be a feature of turbulence under certain assumptions (see Sec. 8.2). The active grid increases the time required to achieve a certain degree of statistical convergence. To reveal the fine details in the inertial range, we develop an approximation of the general shape in the inertial range and use it to detrend 


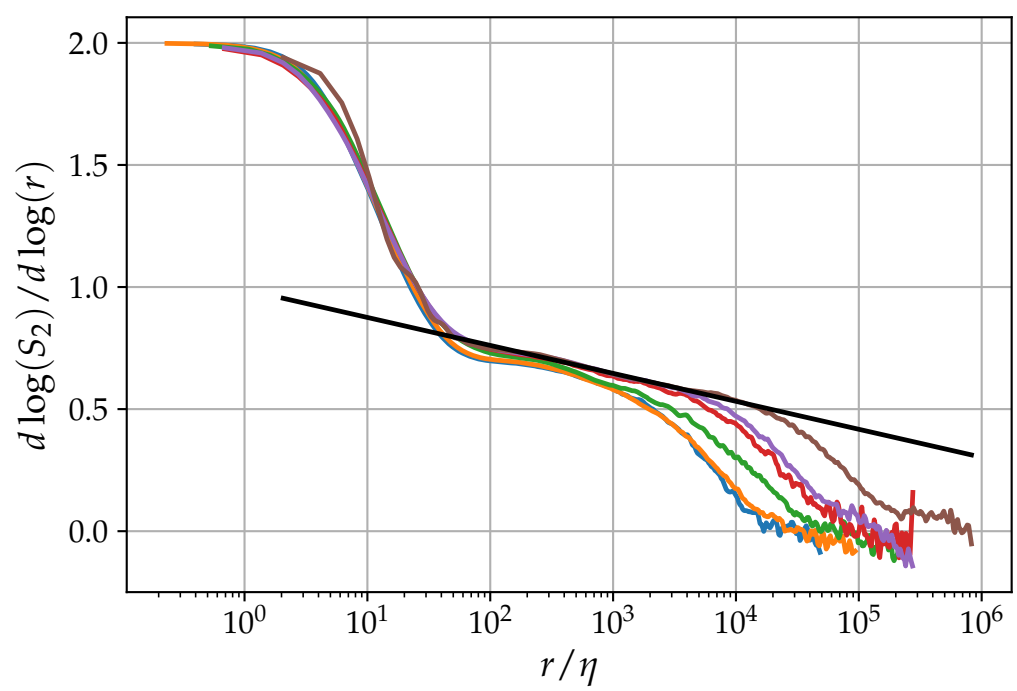

Figure 8.1: Logarithmic derivative of the second-order structure function for selected $R_{\lambda}$ and fit of $a+b \log (r / \eta)$ for the case $R_{\lambda}=5865$. The fit is a good description of this quantity in the inertial range.

the local scaling exponent in the inertial range.

Fig. 8.1 shows the value of the local scaling exponent $\zeta_{2}=d \log \left(S_{2}\right) / d \log (r)$ of the second order structure function for different $R_{\lambda}$. The following analysis makes use of the strikingly simple shape of $\zeta_{2}$ in the inertial range between $100 \eta<r<0.1 L$. Fig. 8.1 shows that in this range of scales, $\zeta_{2}(r)$ follows a line in the log-linear plot, i.e. should be well-described by the functional form $a+b \log (r / \ell)$ (see also Ref. [83]). The parameter $a$ is trivially determined as the value of $\zeta_{2}(r=\ell)$, where $\ell$ is an arbitrary normalisation scale (here: $\left.\eta\right)$. It is shown in Ref. [83], that the slope of the line approaches a constant at high $R_{\lambda}$. Fig. 8.2 shows that the log-linear curve

$$
a_{n}+b_{n} \log (r / \ell)
$$

approximates the general trend in the inertial range rather well also for higher orders $n$.

In the context of this chapter eq. (8.1) will be used to compensate for the general trend of $\zeta_{n}(r)$ in the inertial range and study small features on top of this trend in detail. This should be considered a purely geometrical tool, while the reader is referred to Ref. [51] and Ch. 6 for a more physical, but much more complicated model of the shape of $S_{2}$. The quantity under study is thus

$$
\delta_{n}(r / \eta)=\zeta_{n}(r)-(a+b \log (r / \eta))
$$



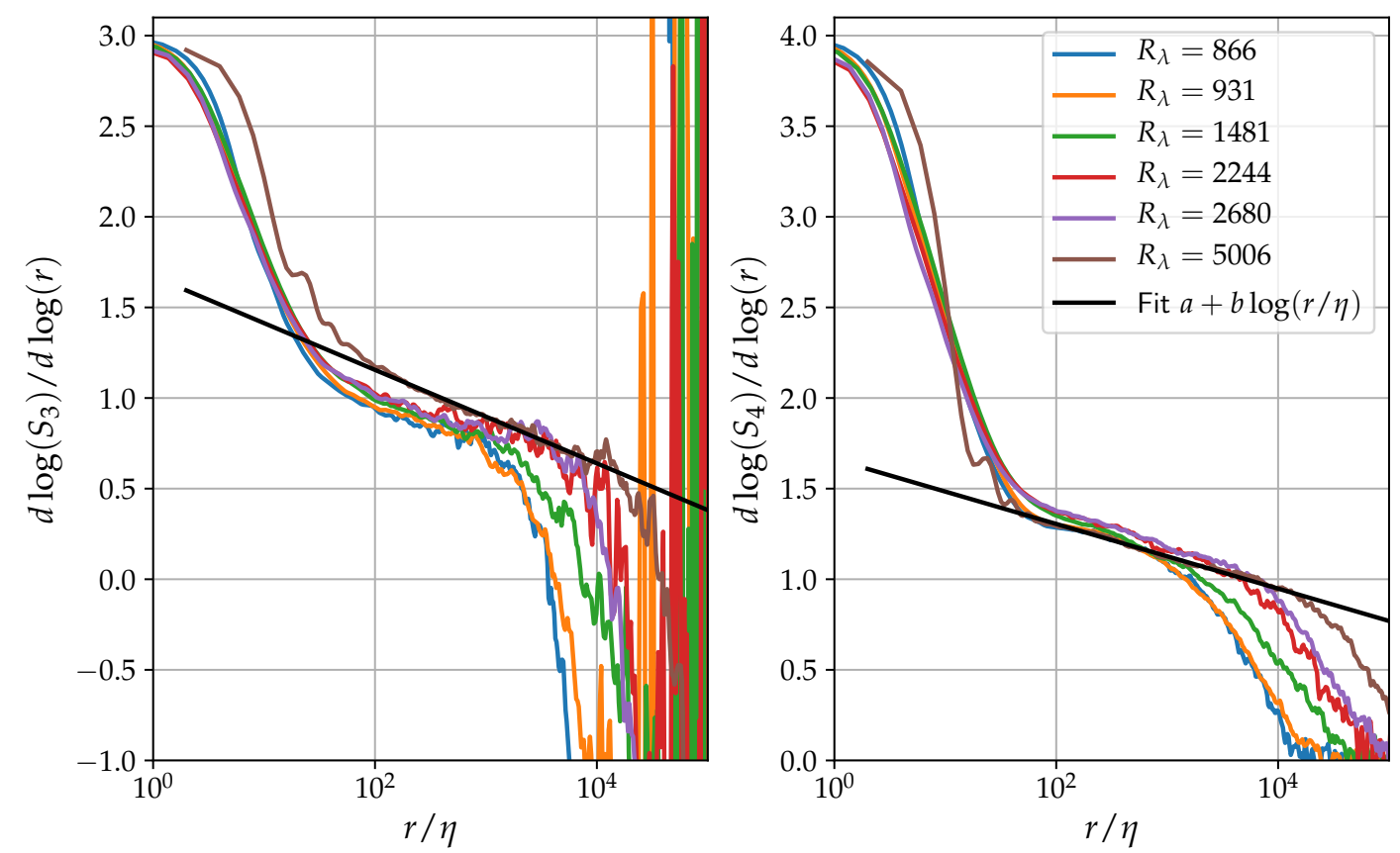

Figure 8.2: Same as Fig. 8.1, but for orders 3 and 4 . The fit is indicated for the higher $R_{\lambda}$ only.

where $a$ and $b$ are fit parameters acquired directly from each measurement of $\zeta_{n}$ by fitting eq. (8.1) to $\zeta_{n}(100 \eta<r<0.1 L)$. This procedure is superior to the study of e.g. $d \log \left(S_{n}\right) / d \log \left(S_{m}\right)$, because it separates the different orders of the structure functions. It further reduces the necessary degree of statistical convergence to distinguish the fine structure from noise. The integral scale $L$ of the data presented here is up to $6 x$ larger than that of the data shown by Sinhuber et al. [81]. As the mean flow was similar to their study, a single time series would have to be about a week long to achieve comparable statistical convergence. In contrast, the time series from which the data presented here was extracted were only 6-8h long. Avoiding the noise-amplifying calculation of $d \log S_{m} / d \log S_{n}$ is thus crucial.

\subsection{Log-Periodic Oscillations in Even-Order Struc- ture Functions}

It has been hypothesised that the structure functions of velocity fluctuations might exhibit small oscillations around the prevailing self-similar scaling. These log-periodic oscillations naturally occur when assuming a selfsimilar process with discrete steps between scales, rather than a continuous scale-dependence $[84,85,86,87]$. Hints of such log-periodic oscillations were found by Anselmet et al. [88] in sixth-order structure functions. Recent exper- 

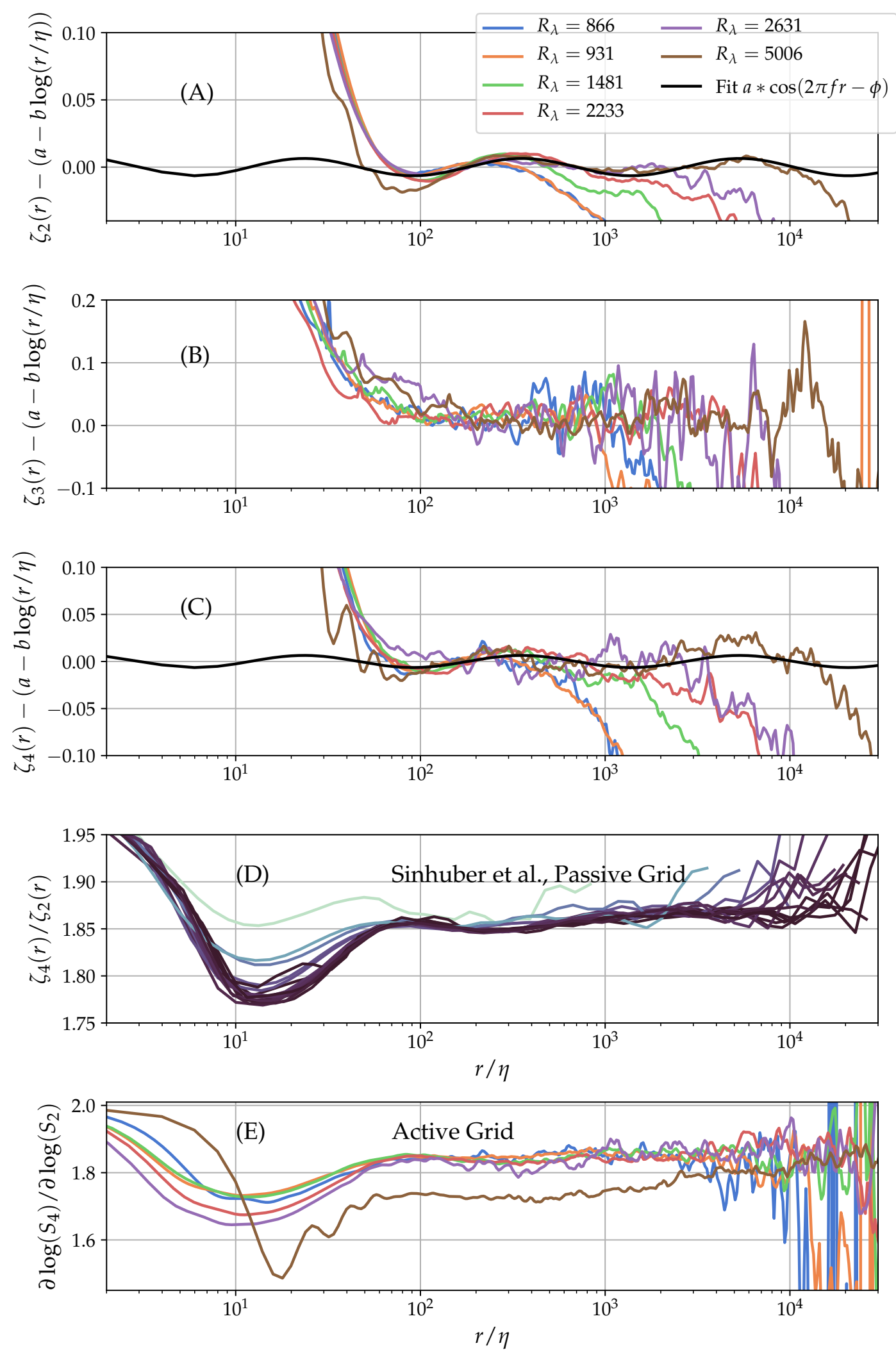

Figure 8.3: (A)-(C) Difference between the local scaling exponent $\zeta_{2}$ and its general trend in the inertial range reveals oscillations for even orders, but not for odd orders. The black curves show fits of the log-periodic cosine (eq. 8.3) to the largest $R_{\lambda}$. (D) and (E) shows the alternative way of extracting these oscillations using ESS-like ratios. The offset of the brown curve in (E) is attributed to instrument effects. The colors in (D) represent $R_{\lambda}$ logarithmically spaced from 100 to 1400 wiht darker colors corresponding to larger Reynolds numbers 
iments of extreme statistical fidelity in the VDTT revealed such a fine structure in plots of $d \log \left(S_{n}\right) / d \log \left(S_{m}\right)$ [81], i.e. the local scaling exponent of ESS plots [89]. The limited Reynolds number and statistical resolution of modern DNS make conclusive statements on the existence of such oscillations difficult. A recent comparison between experimental and numerical data on the matter can be found in Ref. [82].

Fig. 8.3 shows plots of $\delta_{n}(r / \eta)$ for the active grid data and plots of $\zeta_{4}(r) / \zeta_{2}(r)=d \log S_{m} / d \log S_{n}$ from passive grid data of high statistical convergence and active grid data of lower statistical fidelity.

Plot (A) most clearly shows the presence of a fine-structure throughout the inertial range. The number of visible peaks increases with $R_{\lambda}$, while their amplitude saturates at values around o.01. Plot (A) also points towards a constant logarithmic spacing of these peaks, also independent of $R_{\lambda}$.

Plot $(\mathrm{C})$ of $\delta_{4}(r / \eta)$ similarily reveals the the existence of a series of peaks, whose number increase with $R_{\lambda}$. As for $n=2$, the data for $\delta_{4}$ indicates that the position of these peaks is logarithmically spaced. Perhaps owing to the scatter of this data, which has been smoothed by a Konno-OhmachiSmoothing [90, 91], this is not as clear as in plot (A).

Plot (B) of $\delta_{3}$ is at odds to the two even orders, because no periodic features can be distinguished from noise, where again a Konno-Ohmachi-Smoothing was applied. Some of these curves show a peak at the large-scale end of the inertial range. There are indications that this peak is related to the forcing mechanism, because its intensity depends on the active grid configuration.

Plots (D) and (E) show $\zeta_{4}(r) / \zeta_{2}(r)$ as a function of scale $r / \eta$. As pointed out by Sinhuber et al. [81], this quantity equally shows signs of an oscillating fine structure. The most prominent feature in these plots around $\approx 10 \eta$ does not appear in plots $(\mathrm{A})-(\mathrm{C})$. The location of the other local extrema visible in (D) and (E) correspond well to those in (A). The first minimum in (D) is also more pronounced than the extrema at higher $r / \eta$, which appear to have similar heights. Furthermore, this minimum has a clear Reynolds-number dependence and becomes more pronounced with increasing $R_{\lambda}$. This is again at odds with the other extrema of this plot, but owing to the small number of oscillations visible at these rather small $R_{\lambda}<2000$, no conclusive statement can be made.

The first extremum appears to become $R_{\lambda}$-independent in (D), but (E) shows that at the $R_{\lambda}$-dependence extends well beyond the Reynolds numbers studied with a passive grid. Most likely, filtering effects smoothed out the dependence in the passive grid case. This effect can also be observed in 
the active grid data $(\mathrm{E})$ when $\eta$ reaches the size of the sensing element. At this point a comment on the largest $R_{\lambda}$ is necessary. This curve differs from the other measurements from the dissipation range throughout the inertial range. Closer inspection shows that this is due to $\zeta_{4}(r)$, whose value is reduced at the largest $R_{\lambda}$ compared to all other experiments. Higher order moments emphasise the tails of a distribution, in this case the velocity increments. It is hence plausible that higher orders are more challenging for the instrumentation and more prone to related errors. It is nevertheless unsettling that the deviations persist deep into the inertial range.

\subsection{Discussion}

The results indicate that a fine-structure exists in the inertial range that modulates the prevailing trend of scaling statistics of structure functions such as $d \log \left(S_{n}\right) / d \log (r)$ or $d \log S_{m} / d \log S_{n}$. A novel method based on a simple fit is suggested that all allows the extraction of this fine structure. The results are compared to the conventional method [81, 82] based on extended selfsimilarity [73]. The latter method reveals a local minimum around $r / \eta \approx 20$, that the method presented here does not capture. This minimum is clearly outside the inertial range, its amplitude depends on the Reynolds number, and it is a natural outcome of the multifractal $p$-model [92] with a small-scale cutoff [81]. These properties are at odds with all other extrema observable in Fig. 8.3. It is therefore not surprising that a different method to extract the scaling fine structure does not capture this initial oscillation.

The remaining data is consistent with the following picture: The even order structure functions are modulated by oscillations with a logarithmically increasing period and an amplitude that depends neither on scale $r$, or Reynolds number, but increases with order $n$. The data presented here can only show this behaviour up to $n=4$, since measurement noise prevents statements for higher orders $n$. These oscillations are most likely linked to the dissipation range, as pointed out by Sinhuber et al. [81], because the peaks coincide upon normalisation by $\eta$, but not by $L$ (not shown).

Even though only a maximum of two full cycles can be observed in Fig. 8.3, the existence of log-periodic oscillations in even-order structure functions will be taken for granted in the remaining discussion. Such oscillations can be parameterised as

$$
\psi(r)=A \cos (2 \pi f \log (r / \ell))
$$




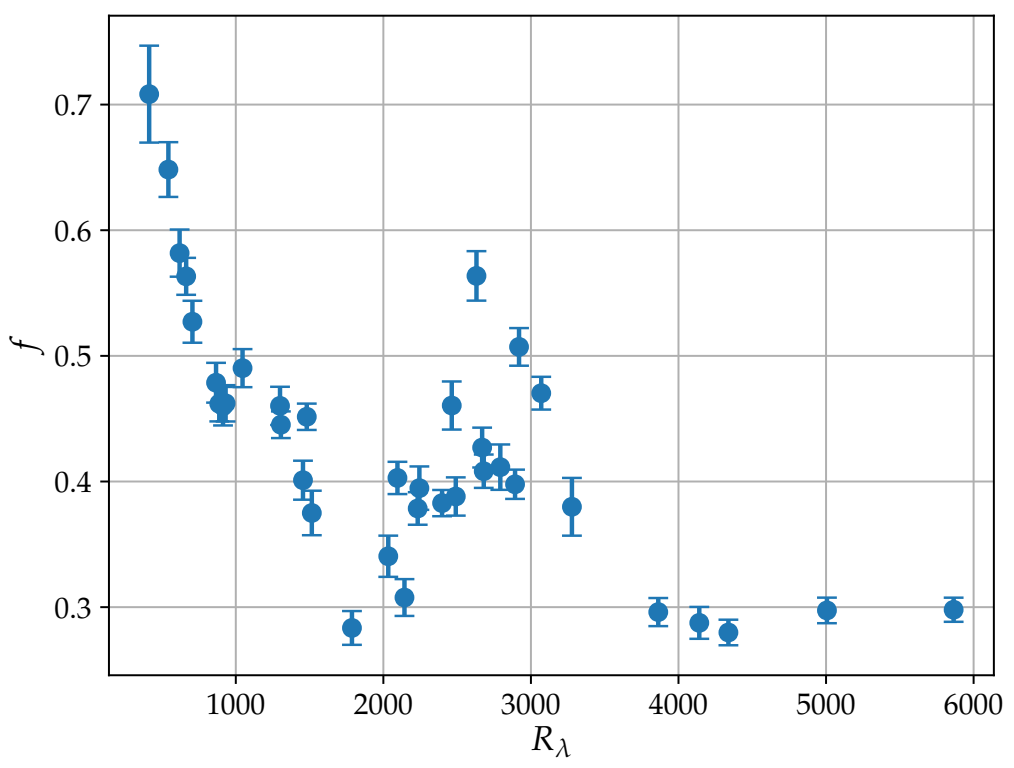

Figure 8.4: Dependence of the logarithmic "frequency" on $R_{\lambda} . f$ appears to assume values between 0.25 and 0.6 corresponding to a scaling factor $\gamma$ between 12 and 30. Error bars mark fit parameter uncertainties.

The parameters $A, f$, and $\ell$ can be obtained by a fit to the data in the range $100 \eta<r<0.1 L$ and the results of such fits are shown in Fig. 8.3 (A) and (C). It can be seen from plot (A) that the parameters of these fits are not expected to change significantly with $R_{\lambda}$, they seem to be universal in this sense. This is confirmed by plotting the extracted values of $f$ vs. $R_{\lambda}$ as in Fig. 8.4. $f$ approaches a value around 0.3 for $R_{\lambda}>2000$, albeit with significant scatter.

According to the discrete scale invariance theory presented in Ref. [93], this frequency is related to a preferred scaling factor $\gamma$, which can be interpreted very loosely as the typical number of eddies that one mother eddy "breaks up" into. If that number is around 2 or 3, but not as large as 10, the log-periodic oscillations are to be expected in statistical objects such as the structure function [86]. Since

$$
\ln (\gamma)=1 / f
$$

$\gamma \approx 12$ for $n=2$ in our data. This is precisely the regime, which Novikov [86] excluded from his prediction of log-periodic corrections to structure functions.

The conclusions of this chapter could therefore be, that undulations to the prevailing scaling of structure functions exist, that these occur in the form of 
logarithmically-spaced peaks, but the spacing of these peak is not as expected originally. The data instead reveals a more than $4 \times$ larger universal scaling factor. This behaviour appears to be limited to even-order structure functions, where only orders 2-4 could be considered. 


\section{Chapter 9}

\section{Experimental Study of the Bottleneck Effect in Fully Developed Turbulence}

This chapter is a reprint with permission of the paper "Experimental Study of the Bottleneck Effect in Fully Developed Turbulence", which appeared in the special issue dedicated to the memory of Pierre Hohenberg of the Journal of Statistical Physics [1]. The author of the thesis performed the measurements, and data analysis and prepared the text. The coauthors organized the research, helped in the interpretation of the data, and edited the manuscript.

[1] C. Küchler, G. Bewley, and E. Bodenschatz. “Experimental Study of the Bottleneck in Fully Developed Turbulence". In: J. Stat. Phys. 175.3-4 (May 2019), pp. 617-639. DoI: 10.1007/s10955-019-02251-1 


\title{
Experimental Study of the Bottleneck in Fully Developed Turbulence
}

\author{
Christian Küchler ${ }^{1,3}$ (D) $\cdot$ Gregory Bewley ${ }^{2}$ Eberhard Bodenschatz ${ }^{1,2,3}$
}

Received: 22 November 2018 / Accepted: 12 February 2019 / Published online: 8 March 2019 (c) The Author(s) 2019

\begin{abstract}
The energy spectrum of incompressible turbulence is known to reveal a pileup of energy at those high wavenumbers where viscous dissipation begins to act. It is called the bottleneck effect (Donzis and Sreenivasan in J Fluid Mech 657:171-188, 2010; Falkovich in Phys Fluids 6:1411-1414, 1994; Frisch et al. in Phys Rev Lett 101:144501, 2008; Kurien et al. in Phys Rev E 69:066313, 2004; Verma and Donzis in Phys A: Math Theor 40:4401-4412, 2007). Based on direct numerical simulations of the incompressible Navier-Stokes equations, results from Donzis and Sreenivasan $(657: 171-188,2010)$ pointed to a power-law decrease of the strength of the bottleneck with increasing intensity of the turbulence, measured by the Taylor micro-scale Reynolds number $R_{\lambda}$. Here we report the first experimental results on the dependence of the amplitude of the bottleneck as a function of $R_{\lambda}$ in a wind-tunnel flow. We used an active grid (Griffin et al. in Control of long-range correlations in turbulence, arXiv:1809.05126, 2019) in the variable density turbulence tunnel (VDTT) (Bodenschatz et al. in Rev Sci Instrum 85:093908, 2014) to reach $R_{\lambda}>5000$, which is unmatched in laboratory flows of decaying turbulence. The VDTT with the active grid permitted us to measure energy spectra from flows of different $R_{\lambda}$, with the small-scale features appearing always at the same frequencies. We relate those spectra recorded to a common reference spectrum, largely eliminating systematic errors which plague hotwire measurements at high frequencies. The data are consistent with a power law for the decrease of the bottleneck strength for the finite range of $R_{\lambda}$ in the experiment.
\end{abstract}

Keywords Turbulence $\cdot$ Fluid dynamics · Anemometry

\section{Introduction}

Turbulence is omnipresent in natural and technological flows. Its consequences for the associated processes are essential in the fields of astrophysics, geophysics, meteorology, biology,

Christian Küchler

christian.kuechler@ds.mpg.de

1 Max-Planck-Institute for Dynamics and Self-Organization, Göttingen, Germany

2 Cornell University, Ithaca, USA

3 Institute for Dynamics of Complex Systems, Georg August University Göttingen, Göttingen, Germany 
and in many engineering disciplines from chemical engineering, combustion science, heat and mass transfer engineering to aeronautics, marine science and renewable energy research. From the fundamental perspective the mathematical field theory of the incompressible Navier Stokes equation continues to challenge pure and applied mathematicians [1]. In turbulence fluid velocities and accelerations fluctuate greatly and any description can only be statistical in nature. It is believed that at very high turbulence levels at spatial scales smaller than the energy injection scale the turbulence shows universal properties, independent of the particular driving. According to Kolmogorov's phenomenology from 1941 [2] (abbreviated K41), the universal statistical spatial properties of fully developed turbulence can be captured in three ranges of spatial scales. Kinetic energy is injected into the turbulent fluctuations at the largest scales, whose properties are particular to the driving mechanism. The kinetic energy is transformed into heat at the very smallest scales through viscous dissipation. If the range of spatial scales found in the turbulent structures is large enough, a third range of scales develops, where neither the peculiarities of energy injection, nor viscous dissipation influence the spatial scale-to scale energy transfer. This range is called the inertial range. In this intermediate range statistical properties can be interpreted by the scale-to-scale transfer of kinetic energy only, described by the kinetic energy dissipation range $\varepsilon$ (dissipated power per unit mass). The dimensionless quantity used to give the strength of turbulence and thus the size of the inertial range scaling is the Taylor microscale Reynolds number

$$
R_{\lambda}=\frac{u \lambda}{v} .
$$

$u$ is the rms of the velocity fluctuations, $v$ is the kinematic viscosity of the fluid, and $\lambda$ is the Taylor microscale, which is a measure for the mean length between two zero-crossings of the velocity fluctuations [3]. $\lambda$ can be thought of as a typical size of an inertial range eddy. In statistically isotropic and homogeneous turbulence $R_{\lambda}$ can be linked to the wellknown Reynolds number $\operatorname{Re}=u L / v$ based on the large scales $L$ via $R_{\lambda}=\sqrt{15 \operatorname{Re}}$ [4]. The integral scale $L$ can be estimated as the integral over the velocity correlation function $L_{11}=\int\langle u(x+r) u(r)\rangle d r$.

In K41 phenomenology for spatially homogeneous and statistically isotropic turbulence the spatial energy spectrum in the inertial range is given by

$$
E(k)=C_{K} \varepsilon^{2 / 3} k^{-5 / 3} .
$$

$C_{K}$ is the Kolmogorov constant, $k$ is the wavenumber. In this K41 spectrum the only free parameter is the dissipation rate $\varepsilon$ as indicated above.

Despite its simplicity, Eq. (1) describes the energy spectrum of observed and simulated turbulent flows quite well (see [5] for a compilation and [6] for an experimental study on the $R_{\lambda}$-dependence of the spectral slope). Nevertheless, important deviations are well known. When analyzing the compensated spectrum $E(k) \varepsilon^{-2 / 3} k^{5 / 3}$, deviations from a $k^{-5 / 3}$ scaling are found. Prominent is an increase in amplitude of the compensated spectrum at the highwavenumber end of the inertial range. This pileup of energy is commonly called the bottleneck effect [7-12]. It has been observed in laboratory flows (e.g. [5,13-15]) and direct numerical simulations (DNS) [16-19] alike and is typically preceded by a distinct local minimum of the compensated spectrum. The bottleneck peak is very shallow or almost absent in hot-wire measurements of atmospheric boundary layer turbulence at very high $R_{\lambda}>10^{4}$ [20-22]. It is generally less pronounced in one-dimensional spectra than in three-dimensional ones [23]. The effect is also present in structure functions and influences the rapidity of the transition between the viscous and inertial ranges in the second-order structure function [19,24], hints of which can also be found in structure functions of higher orders [25]. The most extensive 
analysis of the bottleneck effect has been performed by Donzis and Sreenivasan [19] on DNS at $R_{\lambda}$ up to 1000 . They found that the bottleneck effect can be characterized as the difference between the bottleneck peak height and the level of the preceding minimum in the compensated spectrum. They conclude that the bottleneck effect weakens as a function of $R_{\lambda}$ and report a scaling of $h \sim R_{\lambda}^{-0.04}$. Furthermore, they find that the peak of the bump occurs around $k \eta \approx 0.13$ in three-dimensional spectra, independent of $R_{\lambda}$. Here $\eta$ is the Kolmogorov length scale, where dissipative effects are expected to dominate.

From a theoretical perspective, various explanations exist for the bottleneck effect. Falkovich [10] showed that a small perturbation to a K41 spectrum in the energy transfer equation leads to a correction of the form $\delta E(k)=E(k)\left(k / k_{p}\right)^{-4 / 3} \ln ^{-1}\left(k_{p} / k\right)$, where $k_{p}$ is the bottleneck wavenumber. Kurien et al. [9] argued that the time scale of helicity can be comparable to the energy time scale in the inertial range, where the relative helicity is already weak. They propose that the bottleneck effect is a change in the scaling exponent of the energy spectrum from $-5 / 3$ to $-4 / 3$. Their DNS supports this claim as they find a corresponding scaling range in the three-dimensional spectrum. The scaling is absent in the one-dimensional versions of their spectra. Frisch et al. [8] studied hyperviscous Navier-Stokes equations (Laplacian of order $\alpha \geq 2$ ) and attribute the bottleneck effect to an incomplete thermalization of high-wavenumber modes in the spatial spectrum. None of these studies directly incorporates a $R_{\lambda}$-dependence of the bottleneck height. Verma and Donzis [11] study the nonlocal and nonlinear mode-to-mode energy transfer in a shell model of turbulence and find that a significant portion of the energy flux away from a wavenumber shell goes to distant shells. Thus an efficient energy cascade requires a large inertial range. If the inertial range is insufficient, the energy piles up at the dissipative drop-off. As the length of the inertial range is tightly linked to $R_{\lambda}$, this implies a dependence of the bottleneck intensity on the Reynolds number.

In summary, the bottleneck effect has been studied systematically in DNS and various models. Numerical simulations indicate that the effect gets weaker with increasing $R_{\lambda}$, which is also predicted by Verma and Donzis [11] and in agreement with atmospheric measurements at ultra-high $R_{\lambda}$, where it is absent.

Here we present a detailed analysis of the $R_{\lambda}$-scaling of the bottleneck effect over an unprecedented range of $R_{\lambda}$ in a well controlled laboratory flow. The analysis of the bottleneck effect from experimental data can be demanding as systematic errors can cloud the results. From the perspective of the measuring instrument a small bump in the compensated spectrum is a subtle effect that occurs at rather high frequencies not yet resolvable in PIV or PTV measurements and very difficult to achieve in LDV. We use classical constant temperature hot-wire anemometry (CTA) assuming Taylor frozen flow hypothesis [26] in the Max Planck variable density turbulence tunnel (VDTT) [15]. Even with very well-established hotwire technology, subtle changes in the energy spectrum at high frequencies can be heavily influenced by amplification or attenuation at such frequencies (see Sect. 2.2 for a review).

In this manuscript we work around those effects and investigate the bottleneck effect from the lowest Reynolds number at which it can be identified $(\sim 200)$ up to the highest $R_{\lambda}$ ever measured in a wind tunnel flow.

The paper is organized as follows: first, we present a concise compilation of experimental efforts to reach high $R_{\lambda}$ and describe the variable density turbulence tunnel. We continue with a brief review of challenges posed by constant temperature hot-wire anemometry, especially its frequency responses. In Sect. 3 we introduce the relative spectra that allow us to eliminate instrumentation errors to a large extent. Finally we report the results of our analysis and discuss their relevance for the scaling of the bottleneck effect with $R_{\lambda}$. 


\section{Experimental Methods}

\subsection{High $R_{\lambda}$ and the Variable Density Turbulence Tunnel}

Kolmogorov's 1941 predictions of universal scaling in turbulent flows refer to the limit of large $R_{\lambda}$, such that the regimes of energy injection and viscous dissipation are well separated [2]. This condition is cumbersome to achieve practically. A large separation of scales and therefore a large $R_{\lambda}$ is found in atmospheric flows [20-22], where control is impossible and stationary conditions are difficult to achieve. Flows of high $R_{\lambda}$ are difficult to achieve in controlled laboratory flows, where all scales can be reliably measured. To reach high $R_{\lambda}$ one can turn two knobs: the size of the energy injection scale $L$ and the dissipation scale $\eta=\left(v^{3} / \varepsilon\right)^{1 / 4}$. In direct numerical simulations (DNS), a compromise between the size of the periodoc box, (limiting $L$ ), the spatial and temporal resolution, the convergence time, and the available resources needs to be found [27]. The largest $R_{\lambda}=2340$ achieved in a DNS under these constraints to date has been performed by Ishihara [17]. The limits of computational capabilities in terms of resolution have been recently pointed out by Yeung et al. [27].

In a laboratory experiment the energy injection scale $L$ is limited by the dimensions of the apparatus. Large apparati can be built, e.g. the Modane wind tunnel [28], but are prohibitively expensive to operate, especially considering the many realizations needed for dedicated statistical studies of turbulence. To expand the inertial range the dissipative scales of size $\sim \eta$ can be decreased by lowering the kinematic viscosity $v$ of the working fluid demanding a higher resolution of the measurement instrument. Examples for experiments in liquid helium, which has an ultra-low kinematic viscosity, are found for example in Refs. [29-32]. The authors use liquid helium as working fluid in various flow configurations and have been reported to reach $R_{\lambda}$ up to 10000 . The dissipative scales of these flows are so small that they cannot be resolved by current technology.

Our approach to create a large inertial range is to use a closed-loop wind tunnel filled with sulfur-hexaflouride $\left(\mathrm{SF}_{6}\right)$ at pressures up to 15 bar [15] - the variable density turbulence tunnel (VDTT). With classical grids it has been shown to create $R_{\lambda}$ up to 1600 and Kolmogorov scales $\sim 10 \mu \mathrm{m}$, making even the smallest spatial scales experimentally accessible [33]. With a specially designed autonomous active grid (see below) it is possible to increase the energy injection scale and thus the inertial range. As $R_{\lambda} \sim(L / \eta)^{2 / 3}$, the VDTT features two independent handles to change $R_{\lambda}$-pressure and active grid forcing. In combination they create a laboratory flow of $R_{\lambda}$ more than 5000 at scales resolvable with modern thermal anemometry under the limitations described below.

The autonomous active grid consists of 111 individually controllable flaps of dimensions $11 \mathrm{~cm} \times 11 \mathrm{~cm}$ that rotate around their diagonal. This is different from the Makita-style grids, where the rows and columns of the flaps are mounted rigidly on rotating horizontal and vertical bars [34]. The angle of rotation can be set to any angle between $\pm 90^{\circ}$ The flow obstruction is smallest (flap parallel to the flow) at $0^{\circ}$. At angles $\pm 90^{\circ}$ one of the flap sides is facing the incoming flow, while the other side is facing away from the flow. The sign of the angles determines the side that is facing the flow, while the magnitude defines the deviation of the flap from the parallel position. As in a classical grid with rigid grid bars, wakes are formed that interact with each other downstream of the grid to form a turbulent flow field. The flexibility of the grid allows the superposition of larger structures onto those induced by the individual flaps. A detailed account of the autonomous active grid and the algorithm is given in Ref. [35] and briefly summarized here. A snapshot of several flaps of the autonomous active grid is illustrated in Fig. 1. 


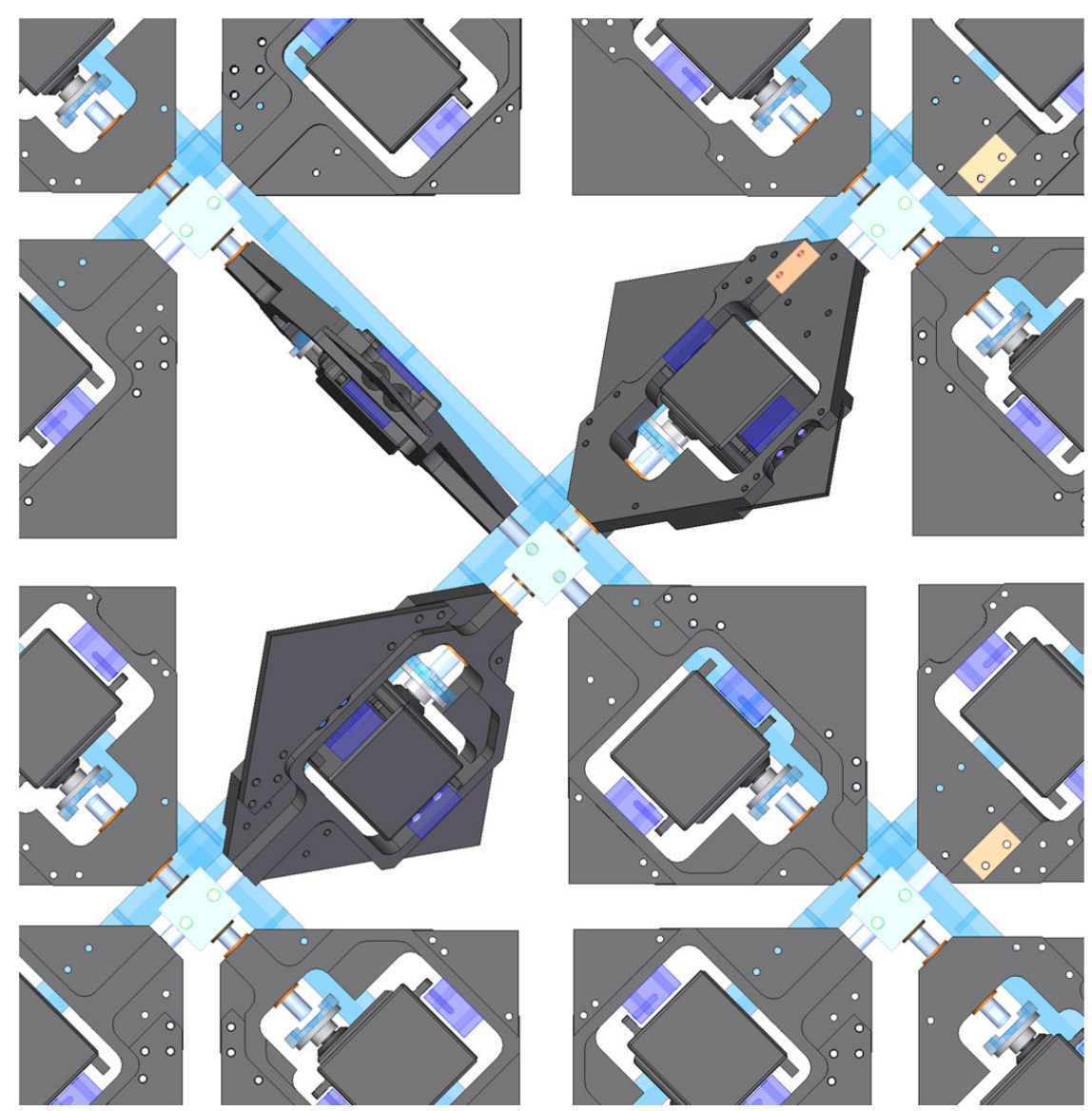

Fig. 1 Several flaps of the active grid. The flow points out of the page. Starting from the top left flap in clockwise direction the flaps are set to $0^{\circ}, 45^{\circ}, 90^{\circ}$, and $45^{\circ}$. The side length of one flap is $11 \mathrm{~cm}$, the black boxes in the flap center are servo motors, the blue rods are the grid support

The algorithm updates the angle of each flap every 0.1s. Each time step starts with a random set of angles and convolves each of those angles with the grid history and a predefined kernel. The kernel is always defined by a certain shape (e.g. Gaussian), the spatial and temporal correlations (the number of neighbors and time-steps included in the convolution), and the desired mean absolute angle $\phi_{R M S}$. For the experiments presented here, a 'Long Tail' kernel has been used, whose description can be found in [35].

This algorithm leads to dynamically evolving patches of more open and more closed flaps without periodicity, which in turn leads to spatial and temporal correlations of the flow structures. The parameters $\sigma_{s}$ and $\sigma_{t}$ describing the correlation lengths that define the grid behavior are typically linked via the mean flow velocity $U$ to avoid a strongly inhomogeneous flow. The grid correlation lengths define the large-scale flow properties. To link these, we consider the overall fluid volume that passes through a typical correlation patch given by $V_{\text {Corr }}=\sigma_{s}^{2} \sigma_{t} U$. The dimensions $L_{\text {Corr }} \approx V_{\text {Corr }}^{1 / 3}$ are proportional to the largest scales in the flow as demonstrated in Fig. 2a. The sine of the mean flap angle $\phi_{\mathrm{RMS}}$ is proportional to the mean area blocked by a single flap. The larger this blockage is, the stronger are the fluctuations induced by the flaps. The product $\sin \left(\Phi_{\mathrm{RMS}}\right) U$ is therefore a predictor for the fluctuating velocity component. The knowledge of typical length scales and velocity defined by the active grid naturally leads to the definition of a Reynolds number using the kinematic viscosity $v$ of the gas.

$$
\operatorname{Re}_{\text {Grid }} \sim \frac{\sqrt[3]{V_{\text {Corr }}} \sin \left(\phi_{R M S}\right) U}{v}
$$



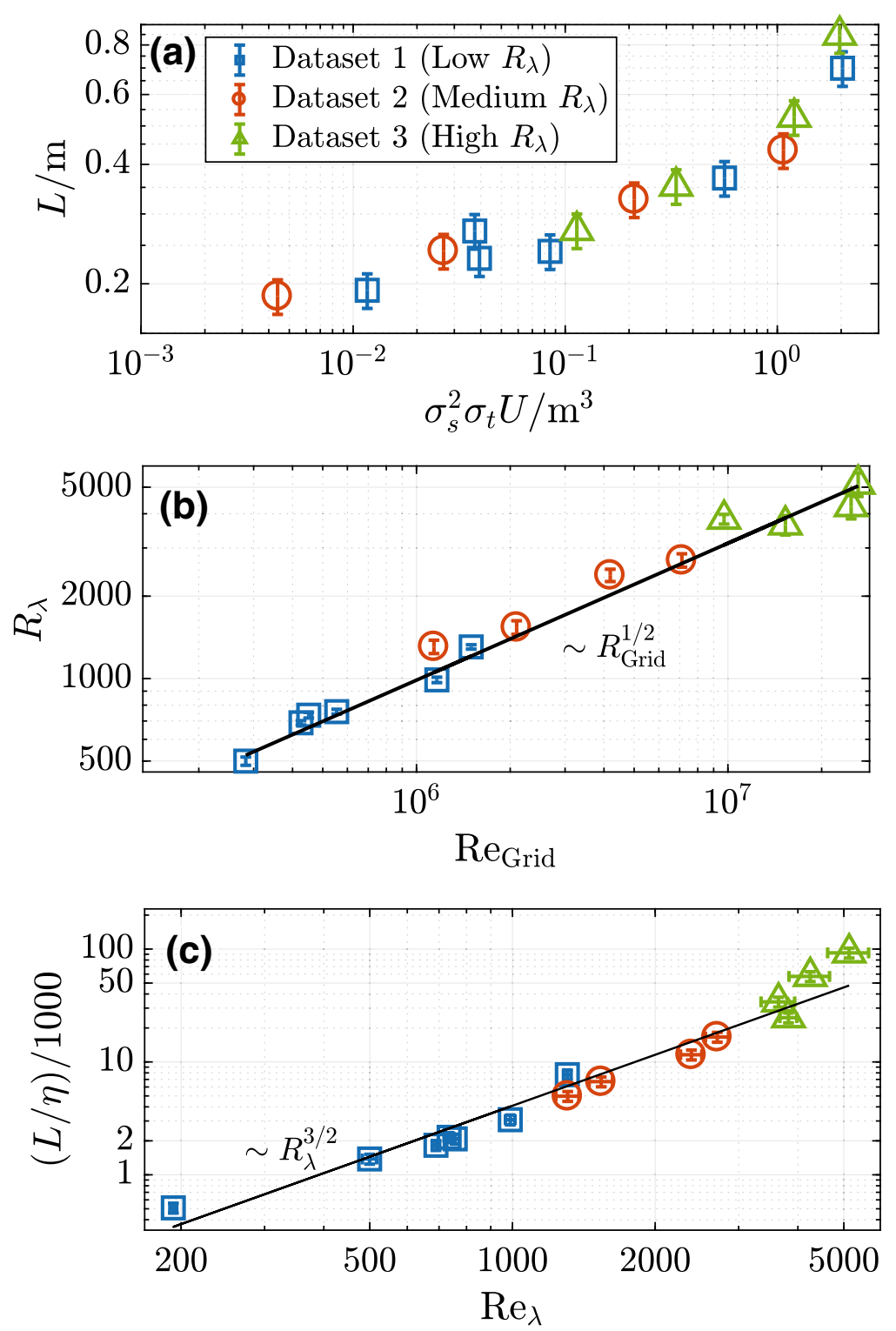

Fig. 2 a The correlation volume $V_{\text {corr }}=\sigma_{s}^{2} \sigma_{t} U$ influences the size of the largest flow scales $L=\int C(r) d r$. b The grid Reynolds number defined as $\operatorname{Re}_{\text {Grid }}=\sqrt[3]{V_{\text {corr }}} \sin \left(\phi_{R M S}\right) U / \nu$ determines $R_{\lambda}$. The black line indicates the isotropic relation $\operatorname{Re}=c \cdot \sqrt{R_{\lambda}}$, where $c$ was chosen to fit the data best. $\mathrm{c}$ The separation of scales $L / \eta$ as a function of $R_{\lambda}$. The black line indicates a best fit of the K41 prediction of $L / \eta \sim R_{\lambda}^{3 / 2}$. We attribute the slight deviations from the scaling to the relation between $L$ and $u^{3} / \varepsilon$, which depends on the large-scale structure of the flow (see Appendix D). The first spectrum of Dataset 1 is not shown in (a) and (b), because $V_{\text {Corr }}$ is not defined for a stationary, open grid

Figure $2 \mathrm{~b}$ shows that the a priori quantity $\operatorname{Re}_{\text {Grid }}$ scales with the a posteriori $R_{\lambda}$ with deviations at $\operatorname{Re}_{\text {Grid }}>10^{9}$. Each Dataset has been obtained by increasing $V_{\text {Corr }}$ while keeping the pressure (and therefore $v$ ) constant as indicated in Table 1. We attribute the slight deviations at large $\operatorname{Re}_{\text {Grid }}$ from a power law dependence to the fact that $L$ is approaching half the diameter of the measurement section because the rule-of-thumb $\sigma_{s} \approx \sigma_{t} \cdot U$ is relaxed slightly. This is a natural limit for a sensible energy injection in any tunnel. We would like to add the word of caution that when approaching this limit, isotropy and homogeneity cannot be assumed easily anymore, which leads to said deviations from the isotropic relation $R_{\lambda} \sim \operatorname{Re}_{\text {Grid }}^{\zeta}$ with $\zeta \approx 0.5$ (black line in Fig. 2b). This effect can also be observed in Fig. 2c). When approximating $L=\int_{0}^{\infty} C(r) d r$, deviations from the K41 prediction $L / \eta \sim R_{\lambda}^{3 / 2}$ appear. The scaling is recovered when calculating $L=C_{\varepsilon} u^{3} / \varepsilon$ (not shown). We attribute 
Table 1 Properties of all spectra

\begin{tabular}{|c|c|c|c|c|c|c|cc|}
\hline Dataset & $R_{\lambda}$ & $p($ bar $)$ & $U(\mathrm{~m} / \mathrm{s})$ & $u(\mathrm{~m} / \mathrm{s})$ & Sensor & $L=\int C(r) d r(\mathrm{~m})$ & $\eta(\mu \mathrm{m})$ & $f_{\eta}(\mathrm{kHz})$ \\
\hline \hline 1 & 193 & 1.5 & 2.75 & 0.04 & Regular HW & 0.12 & 277 & 9.9 \\
\hline 1 & 500 & 1.5 & 2.41 & 0.14 & Regular HW & 0.19 & 139 & 17.3 \\
\hline 1 & 690 & 1.5 & 2.41 & 0.18 & Regular HW & 0.23 & 127 & 19.0 \\
\hline 1 & 735 & 1.5 & 2.65 & 0.19 & Regular HW & 0.27 & 127 & 20.9 \\
\hline 1 & 757 & 1.5 & 2.43 & 0.20 & Regular HW & 0.24 & 116 & 20.9 \\
\hline 1 & 989 & 1.5 & 2.72 & 0.22 & Regular HW & 0.32 & 120 & 22.7 \\
\hline $\mathbf{1}$ & $\mathbf{1 3 0 5}$ & $\mathbf{1 . 5}$ & $\mathbf{2 . 2 8}$ & $\mathbf{0 . 3 4}$ & Regular HW & $\mathbf{0 . 7 0}$ & $\mathbf{9 1}$ & $\mathbf{2 5 . 1}$ \\
\hline $\mathbf{2}$ & $\mathbf{1 3 0 8}$ & $\mathbf{5 . 9 5}$ & $\mathbf{3 . 6 4}$ & $\mathbf{0 . 2 0}$ & NSTAP 1 & $\mathbf{0 . 1 9}$ & $\mathbf{3 7}$ & $\mathbf{9 8 . 4}$ \\
\hline 2 & 1539 & 5.95 & 3.68 & 0.22 & NSTAP 1 & 0.24 & 36 & 102.2 \\
\hline 2 & 2385 & 5.97 & 3.64 & 0.35 & NSTAP 1 & 0.33 & 28 & 130.0 \\
\hline 2 & 2704 & 5.97 & 3.61 & 0.40 & NSTAP 1 & 0.43 & 26 & 138.9 \\
\hline \hline 3 & 3641 & 14.62 & 3.75 & 0.44 & NSTAP 2 & 0.38 & 10 & 375.0 \\
\hline 3 & 3821 & 14.71 & 3.83 & 0.41 & NSTAP 2 & 0.27 & 12 & 319.2 \\
\hline $\mathbf{3}$ & $\mathbf{4 2 4 7}$ & $\mathbf{1 4 . 6 5}$ & $\mathbf{3 . 9 7}$ & $\mathbf{0 . 5 3}$ & NSTAP 2 & $\mathbf{0 . 5 4}$ & $\mathbf{9 . 2}$ & $\mathbf{4 3 1 . 5}$ \\
\hline 3 & 5130 & 14.66 & 4.01 & 0.58 & NSTAP 2 & 0.86 & 9.1 & 440.7 \\
\hline
\end{tabular}

All spectra of a Dataset are distorted by the same function $T(f)$ describing the sensor- and instrumentinduced bias. This is ensured by changing $R_{\lambda}$ only through large scales $L$ and fixing the position of the small scales in frequency space indicated by $f_{\eta}$. A reference spectrum has been chosen from each dataset, which is emboldened in this table

this to differences in $C_{\varepsilon}$ due to the turbulence forcing as discussed e.g. in Refs. [36-39] and plotted for our experiments in the appendix. From a phenomenological of view, introducing correlations into the flow using the active grid increases $R_{\lambda}$ only up to a certain limit (probably related to the tunnel dimensions). When exceeding this limit, the integral length scale $L$ can no longer be estimated by the correlation length. Nevertheless, these data confirm that the active grid is indeed another 'knob' to change $R_{\lambda}$ through the largest scales.

\subsection{Thermal Anemometry}

More than a century after its invention [40], hot-wire anemometry remains the technique of choice to measure the energy spectrum of turbulent velocity fluctuations in a strong mean flow. Constant temperature anemometry is responsive to fluctuations up to very high frequencies. The sensing element's resistance-and therefore its temperature-is kept constant by a feedback circuit. As long as the feedback circuit is fast enough, the thermal lag of the wire does not attenuate fluctuations faster than the thermal time scale of the wire. This comes at the expense of a more complicated circuitry and frequency response.

The frequency response of CTA circuits has been studied extensively both through theoretical models and experimental testing. Freymuth [41] linearized a circuit with a single feedback amplifier of infinitely flat frequency response and analyzed its response to square and sine waves. He finds that the system can be modeled by a third-order ODE if the circuit responds faster than the wire, and the frequency response is optimal (flat over the entire range of frequencies) when the system response to a step perturbation by a single, slight overshoot (critically damped system). Perry and Morrison [42] investigated more moderate amplifier gains and bridge imbalances in their study yielding similar results. Wood [43] expanded the Perry and Morrison analysis, but considered a single-stage amplifier with a frequency-dependent response. Watmuff [44] further expanded the model with multiple, nonideal amplifier stages. He showed that at least two amplifier stages are necessary to model the real amplifier properly. This introduces two additional poles to the system and makes the frequency response more complicated. Samie et al. [45] recently studied anemometry with sub-miniature probes in this model and compared it to a real CTA measurement. The results 
supported the further development of their in-house circuit, such that sub-miniature hot wire probes could be operated successfully on this CTA for the first time.

These theoretical attempts to predict the frequency response of a CTA circuit are accompanied by experimental approaches. Bonnet and de Roquefort [46] heated the wire periodically by a perturbation voltage as well as laser heating to determing the frequency response. Weiss et al. [47] used the aforementioned square wave test and interpreted its power spectrum as a measure for the frequency response curve. Hutchins et al. [48] exploited the well-defined frequency content of pipe flow at different operating pressures to obtain frequency response curves without artificial heating. They were able to create flows of almost identical Reynolds number, but different frequency content and could deduce the frequency-response curves for different circuits and wires. They compared several anemometer circuits and wires and found that the frequency responses are non-constant at frequencies as low as $500 \mathrm{~Hz}$. For the combination of CTA circuit and wire used in the present study, they report an attenuation between $400 \mathrm{~Hz}$ and $7 \mathrm{kHz}$ followed by a strong amplification of the signal. We therefore cannot assume a flat frequency response for our measurements and adress these effects below.

The energy spectrum measured by a hot wire is influenced by the effects of finite wire length. Length scales smaller than the sensor's sensing lengths $l$ will be attenuated, but also larger wavenumbers are influenced. Wyngaard [49] used a Pao model spectrum [50] to investigate this attenuation of small scales. These results were reviewed in Ref. [51] indicating that for $l / \eta=2$, the attenuation of the one-dimensional spectrum is still minimal at $k \eta \sim 0.3$, which was supported by Ashok et al. [52]. Sadeghi et al. [53] used sub-miniature hot wires (NSTAPs) as a benchmark and found that spatial filtering of the energy spectrum is minimal for $l / \eta<3.7$ at $k \eta<0.1$.

In this study we used conventional hot wires of sensing length $450 \mu \mathrm{m}$ for pressures below 2 bar, as well as nanoscale thermal anemometry probes (NSTAP) of sensing length $30 \mu \mathrm{m}$ provided by Princeton University with a Dantec Dynamics StreamWare CTA circuit. The NSTAP is a $100 \mathrm{~nm}$ thick, $2.5 \mu \mathrm{m}$ wide, and 30 or $60 \mu \mathrm{m}$ long free-standing platinum film supported by a silicon structure and soldered to the prongs of a Dantec hot wire. The production process and characteristics are detailed in Refs. [54-57]. For the conventional hot wire $l / \eta<5$ in all cases and for the NSTAP $l / \eta<3$. Therefore, $\eta$ cannot be fully resolved in all cases. However, the bottleneck effect is typically found around $100 \eta$. The aforementioned references show that we can regard the distortions due to finite wire length as minor in this part of the energy spectrum.

To summarize, the spatial resolution of our measurement instruments is sufficient to study the $R_{\lambda}$-dependence of the bottleneck effect. Nevertheless the nonlinear frequency response of the circuitry remains a source of systematic error that is different from random noise occuring at very high frequencies. Here we describe a procedure that takes the response into account and thus removes this systematic measurement error.

\section{Relative Spectra}

\subsection{The Concept}

As outlined above, systematic errors influence the energy spectra recorded with a hot-wire anemometer. Formally, this means that the one-dimensional energy spectrum $E_{11}(f)$ is distorted by a frequency-dependent transfer function $T(f)$ :

$$
E_{M}(f)=E_{11}(f) T(f)
$$


$T(f)$ describes the effects of the thermal wire response, which depends on pressure and speed, and the reponse of the constant temperature anemometry circuit. Ideally, $T(f)$ is a constant over the whole range of relevant frequencies, but the evidence detailed above indicates a complex shape of amplification and attenuation of the signal. In this study we do not make any attempt to find $T(f)$. Instead, we control its effects by keeping $T(f)$ the same for several flows at different $R_{\lambda}$.

To ensure that the spectra only differ because of changes in the turbulent fluctuation and not because of the frequency response curve of the anemometer, we need to ensure that the response curve $T(f)$ is unaltered between spectra. We achieve this in two steps. The ambient pressure might influence the heat transfer of the wire and therefore $T(f)$. Furthermore, $T(f)$ is influenced by the CTA tuning (in particular the overheat), and the sensor itself. Therefore, we fix the ambient pressure within a set of spectra (a 'Dataset') and measure using the same sensor and the same CTA settings.

The second step is to ensure that a given $k \eta$ is influenced by the same part of the frequency response curve $T(f)$. Thus, we need to fix the position of a spectral feature in frequency space. This means that the mean velocity $U$ must be the same within one Dataset. $T(f)$ mainly distorts the small-scale end of the spectrum [41-45,47,48,51], whose location in frequency space at a given $U$ is determined by the kinematic viscosity $v . v$ is fixed within a Dataset because the pressure remains constant.

We can, however, change the energy injection scale and thus $R_{\lambda}$ with the autonomous active grid. This way we can conduct measurements at different $R_{\lambda}$. Ultimately, we can eliminate $T(f)$ by relating each spectrum to a reference spectrum:

$$
\begin{aligned}
\frac{E_{M}^{i}(f)}{E_{M}^{\operatorname{Ref}}(f)} & =\frac{E_{11}^{i}(f) T^{i}(f)}{E_{11}^{\operatorname{Ref}}(f) T^{\operatorname{Ref}}(f)} \\
& =\frac{E_{11}^{i}(f) T(f)}{E_{11}^{\operatorname{Ref}}(f) T(f)}=\frac{E_{11}^{i}(k U / 2 \pi)}{E_{11}^{\operatorname{Ref}}(k U / 2 \pi)} .
\end{aligned}
$$

In the following we call the ratio of a spectra divided by a reference spectrum in the frequncy domain, relative spectrum. We emphasize that the notion of relative spectra is not necessary to investigate $R_{\lambda}$-scaling within one Dataset prepared in the aforementioned way. However, the accessible range of $R_{\lambda}$ by changing only the active grid forcing is limited. Therefore, several of such Datasets with different frequency distortions need to be prepared by changing kinematic viscosity $v$ and mean flow speed $U$ to obtain a convincing scaling range. The notion of relative spectra is then required to compare those Datasets.

\subsection{Results}

We created three sets of spectra that have identical $T(f)$ each. We call these sets 'Datasets'. Table 1 shows important parameters for each spectrum. Note that $L$ changes significantly within a given dataset leading to changes in $R_{\lambda}$, while $f_{\eta}=U / \eta$ remains almost constant within the dataset. This indicates that we changed $R_{\lambda}$ only by increasing the large scales, while keeping all small-scale features of the spectrum at the same frequency. For example, in Dataset 2, the peak of the spectral bump always lies at a frequency of $\sim 700 \mathrm{~Hz}$, whereas the beginning of the inertial range spans a factor of 4 in frequency ( 2 to $8 \mathrm{~Hz}$ ). This exemplifies the excellent control over $R_{\lambda}$ permitted by the autonomous active grid as indicated in Fig. 2 .

The lower graphs of Figs. 3, 4 and 5 show the spectra from each of the respective datasets divided by the reference spectrum $E_{11}^{\mathrm{Ref}} \cdot E_{M}^{\mathrm{Ref}}$ is plotted pre-compensated in the upper graphs 


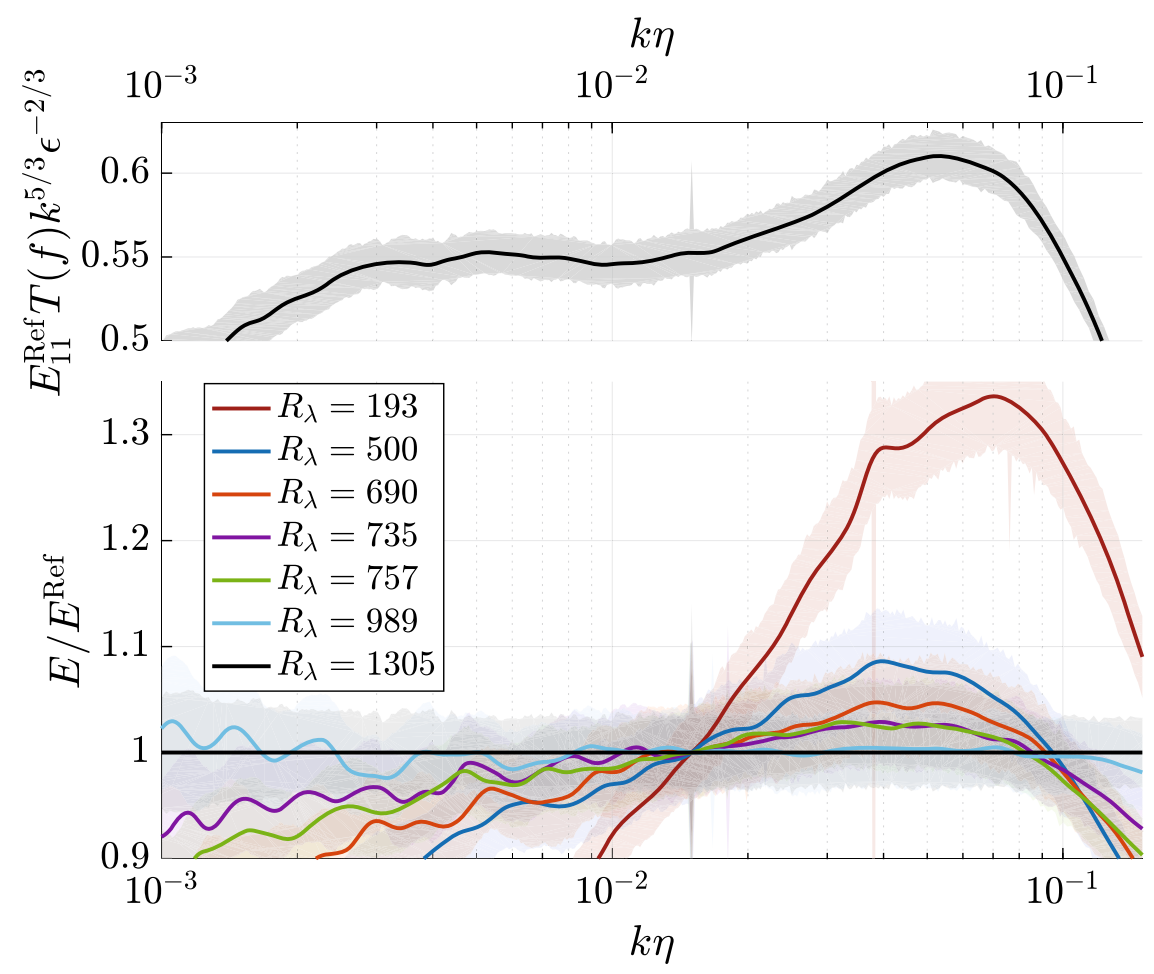

Fig. 3 Reference spectrum at $R_{\lambda}=1305$ (upper plot) and relative spectra from Dataset 1 . The data have been collapsed at $k \eta=0.015$, which we defined as the beginning of the bottleneck region. We identified the peaks in the relative spectra with the bottleneck peak of the absolute spectra. The peak height decreased with increasing $R_{\lambda}$ and different spectra of similar $R_{\lambda}$ result in very similar relative spectra as expected. Furthermore, the slope of the spectrum at $k \eta<0.015$ seems to decrease with $R_{\lambda}$. The shaded areas are a measure of the noise level

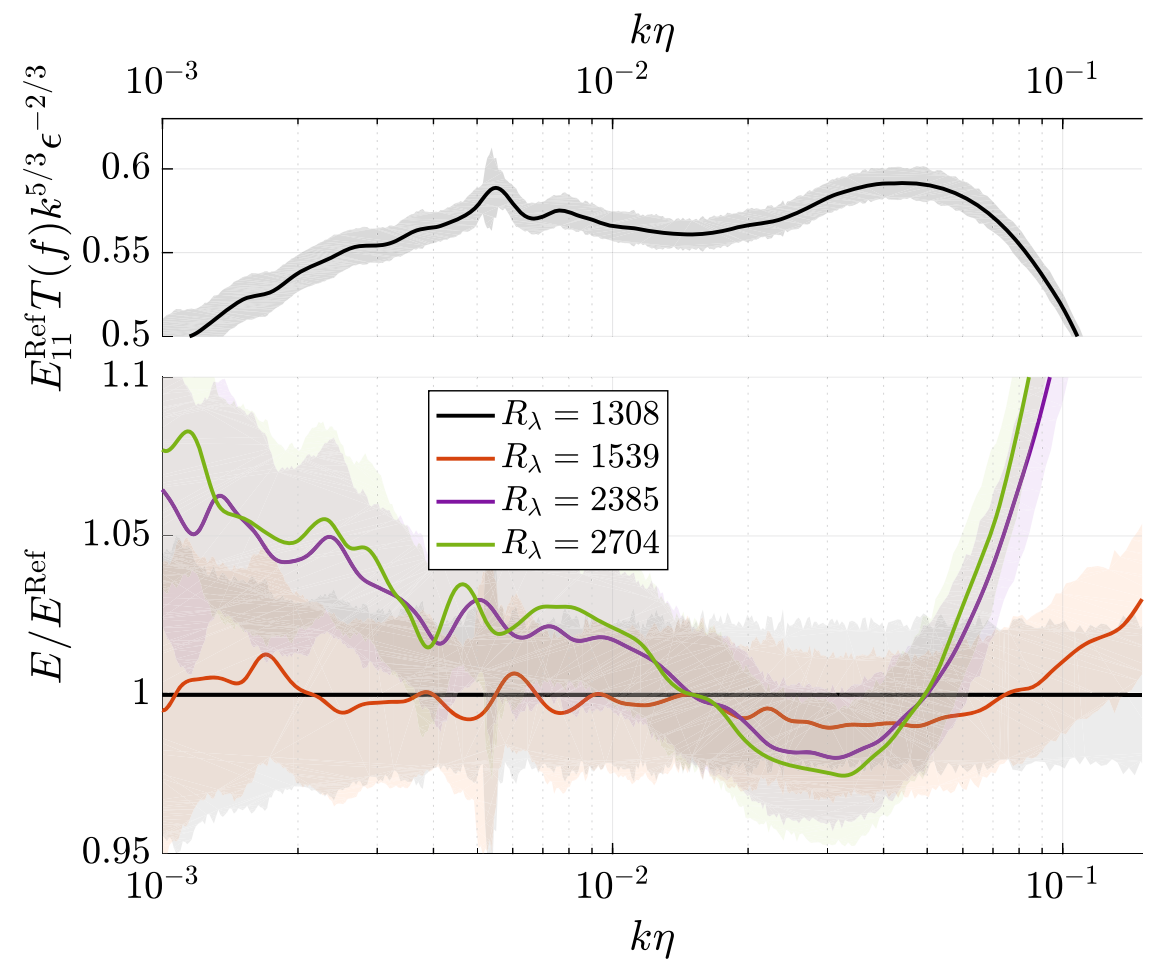

Fig. 4 Reference spectrum at $R_{\lambda}=1308$ (upper plot) and relative spectra from Dataset 2 . The trends in peak height and slope from Fig. 3 continue 


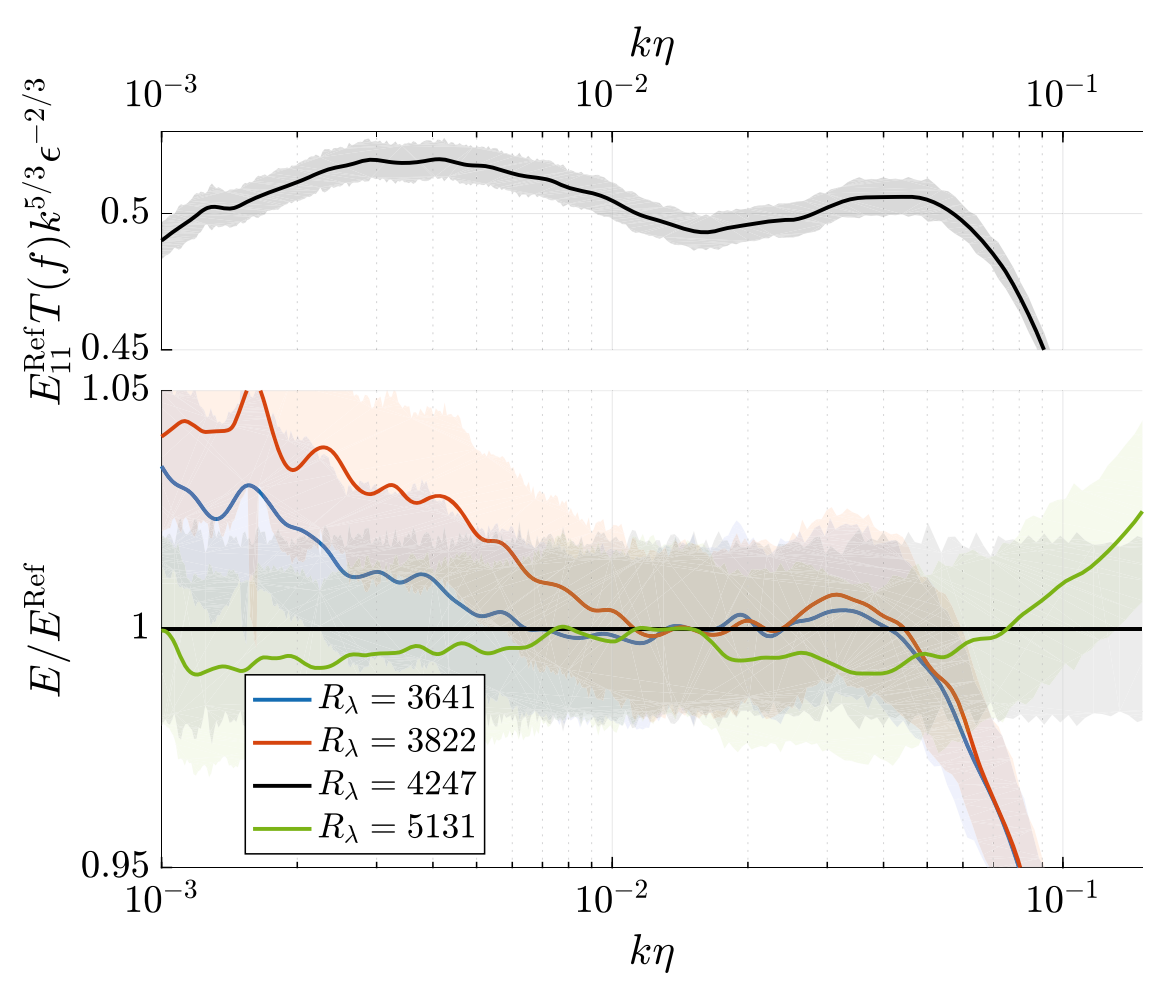

Fig. 5 Reference spectrum at $R_{\lambda}=4247$ (upper plot) and relative spectra from Dataset 3. Unlike in Datasets 1 and 2, the beginning of the bottleneck region around $k \eta=0.015$ is identifiable in the relative spectra as a local extremum

of the respective figure. Note that the absolute spectra in the upper graphs are multiplied by an unknown transfer function $T(f)$ accounting for probe effects and therefore can not be used to reliably measure the features of the bottleneck. However, the relative curves are corrected and allow a measurement. The graphs are the result of a smoothing procedure and error estimate detailed in the appendix. In brief, the spectra were smoothed using a $1 / f$ octave filtering and the error is related to the noise level removed by the smoothing procedure. The spectra have been divided by the reference spectrum in the frequency domain and collapsed at $k \eta=0.015$ afterwards to simplify interpretation. This is the approximate location of the local minimum regularly found in compensated spectra and directly precedes the bottleneck peak in wavenumber space. DNS studies by Donzis and Sreenivasan [19] and Ishihara et al. [17] have shown that the magnitude of the compensated spectrum at $k \eta=0.015$ is practically independent of $R_{\lambda}$. It is difficult to achieve such a good collapse of compensated spectra in experimental data, because of uncertainties in the measurement of the dissipation rate $\varepsilon$. Donzis and Sreenivasan [19] regard the compensated spectrum around $k \eta=0.015$ as the true measure of the Kolmogorov constant and build their analysis of the bottleneck effect on the difference between the local minimum and the bottleneck peak. By normalizing the relative spectra at this point we follow their procedure and remove the effects of imperfect estimates of $\varepsilon$. In other words, we define the bottleneck height as the relative departure of the compensated spectrum from its magnitude at $k \eta=0.015$ following the procedure described in Ref. [19]. The remainder of the analysis is carried out on these relative spectra normalized at $k \eta=0.015$.

While in Dataset 3 the beginning of the bottleneck region around $k \eta=0.015$ is accompanied by a change in the shape of the relative spectra, this point cannot be identified in the relative spectra of Datasets 1 and 2. In the following we concentrate on the bottleneck effect found at $k \eta>0.015$ for the remainder of this section. 
The location of the spectral bump forming the bottleneck effect in relative spectra is not obvious. Our data from one-dimensional spectra suggests that the peak occurs between $k \eta=0.03$ and $k \eta=0.06$, which is consistent with the findings from DNS, where the peak typically occurs at $k \eta=0.046$ in the one-dimensional spectra (see e.g. Refs. [19], [17]). However, when considering the background noise, the peak location is not the major source of error. E.g. for $R_{\lambda}=1539$, all points between $0.015<k \eta<0.07$ are within the errorband at $k \eta=0.05$. We therefore define the extremum in the relative spectrum between $0.015<k \eta<0.08$ as the relative height $h$ of the bottleneck effect. This has the additional advantage to be independent of the errors in the estimate of $\eta$. To preclude biases from this definition, we repeat our analysis with different definitions of the relative bottleneck height in Fig. 11 in the appendix.

Finally, the measured bottleneck height cannot depend on which spectrum is chosen as reference. We have calculated the bottleneck height with all possible choices of $E_{11}^{\text {Ref }}$ and found our results to be largely independent of that choice (see Appendix for details).

Figure 6 shows the bottleneck height—defined as above- as a function of $R_{\lambda} / R_{\lambda}^{\text {Ref }}$ within each dataset. The data shows a trend towards smaller peak heights in the relative spectrum with increasing $R_{\lambda}$. The data follows the numerical data we have compiled from various sources [17,58-60]. We have analyzed the data from Buaria et al. [60] at $R_{\lambda}$ up to $1000\left(R_{\lambda} / R_{\lambda}^{\text {Ref }}<1\right)$. The increased small-scale resolution in comparison to [19] seems to have no noticable impact on the bottleneck. Therefore, this data at is practically the same as the one used by Donzis $\&$ Sreenivasan [19] for our purposes. The data from $R_{\lambda}=1300\left(R_{\lambda} / R_{\lambda}^{\text {Ref }}=1\right)$ was reported in Ref. [58]. The numerical data at $R_{\lambda} / R_{\lambda}^{\text {Ref }} \approx 1.9$, which corresponds to $R_{\lambda}=2340$, is the highest $R_{\lambda}$ reported by Ishihara et al. [17]. The relative spectra of the numerical data were analyzed equivalently to the experimental data and the spectrum at $R_{\lambda}=1300$ was chosen as a reference spectrum. We have used the one-dimensional spectra in our analysis of the numerical data.

When excluding the lowest $R_{\lambda}$, the experimental data is in agreement with the power law of

$$
h \sim\left(R_{\lambda} / R_{\lambda}^{\mathrm{Ref}}\right)^{-0.061 \pm 0.007} .
$$

The fit was obtained by a bootstrap procedure based on the error bars. It compares well with the findings of Donzis and Sreenivasan [19], who report a bottleneck scaling of $h \sim R_{\lambda}^{-0.04}$. Their analysis similarly defines the bottleneck height as the difference between the height of the compensated spectrum at the bottleneck peak and the local minimum preceding this peak.

The spectrum at $R_{\lambda}=193$ follows the general trend of decreasing peak height with $R_{\lambda}$, but its peak differs substantially from the predictions. The absolute spectrum (not shown) exhibits no signs of a 5/3-scaling, and consequently the bottleneck region cannot be clearly separated from the rest of the spectrum. This is substantially different from the other spectra, where the end of the integral range could always be observed in the absolute spectra and we therefore are not surprised that the relative spectrum at $R_{\lambda}=193$ deviates from the remainder of the data. This spectrum has consequently been ignored in our interpretation.

Further, we can change $R_{\lambda}$ only by a factor of 5 through the autonomous active grid. While Dataset 1 and 2 each feature a spectrum at the same $R_{\lambda}$, there is a gap between the highest $R_{\lambda}$ of Dataset 2 (2704) and the lowest of Dataset 3 (3641). To plot $h$ as a function of $R_{\lambda}$ alone, we use the aforementioned power law fit from Fig. 6. Under this assumption we can bridge the gap between the two datasets, because $h_{1} / h_{2}=\left(R_{\lambda 1} / R_{\lambda 2}\right)^{-0.0061 \pm 0.007}$. Using the final point of Dataset 2 as $R_{\lambda 1}=2704$ and the corresponding height $h_{1}$ we can construct $h_{2}$ using the lowest $R_{\lambda 2}=3641$ from Dataset 3 to arrive at Fig. 7 


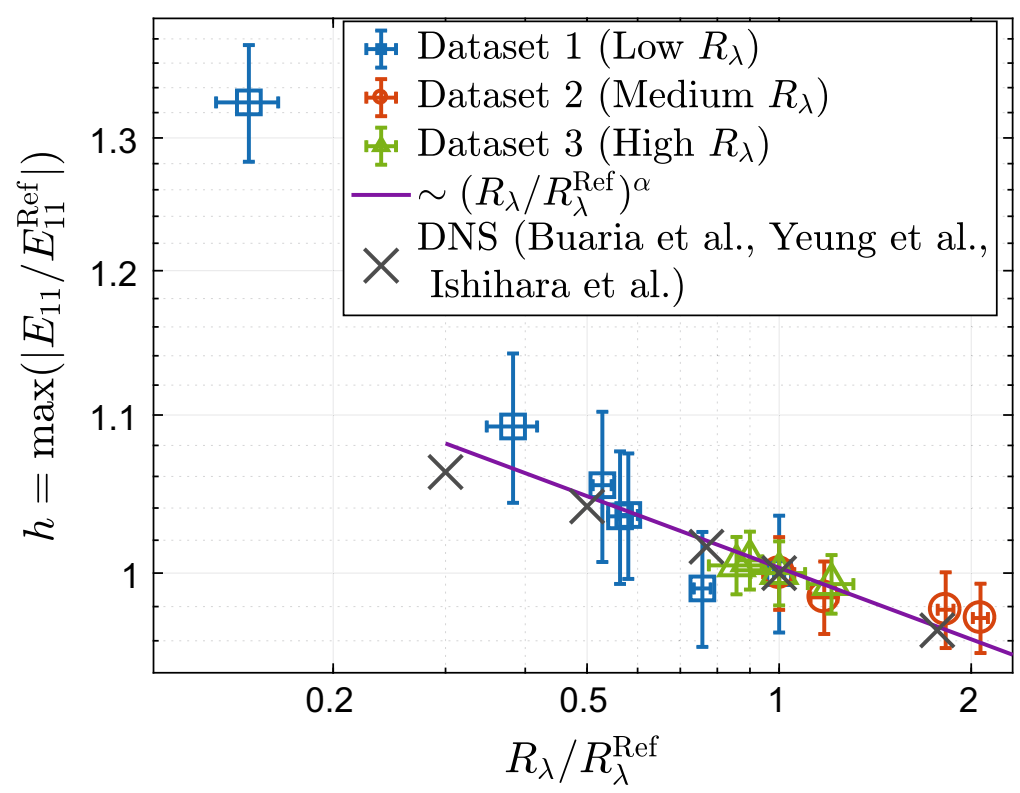

Fig. 6 Bottleneck height relative to the corresponding reference spectra for all datasets as a function of $R_{\lambda} / R_{\lambda}^{\text {Ref }}$. The reference spectra collapse at $R_{\lambda} / R_{\lambda}^{\text {Ref }}=1$ and have a relative bottleneck height of 1 by construction. Numerical simulations from Buaria et al. [60] at $R_{\lambda}$ up to $1000\left(R_{\lambda} / R_{\lambda}^{\text {Ref }}<1\right)$, Buaria et al. [58] $\left(R_{\lambda}=1\right)$ and Ishihara [17] $\left(R_{\lambda}>1\right)$ are added for reference. The DNS data for $R_{\lambda}<2000$ is practically identical to the data analyzed by Donzis and Sreenivasan [19]. A power law is fitted to the experimental data with the lowest $R_{\lambda} / R_{\lambda}^{\mathrm{Ref}}$ excluded. $\left(R_{\lambda} / R_{\lambda}^{\mathrm{Ref}}\right)^{-0.061 \pm 0.007}$ is a good description of the experimental data over one decade of $R_{\lambda}$ (from 500 to 5000) and agrees with the numerical simulations as well. This power law is used in Fig. 7 to combine Datasets 2 and 3

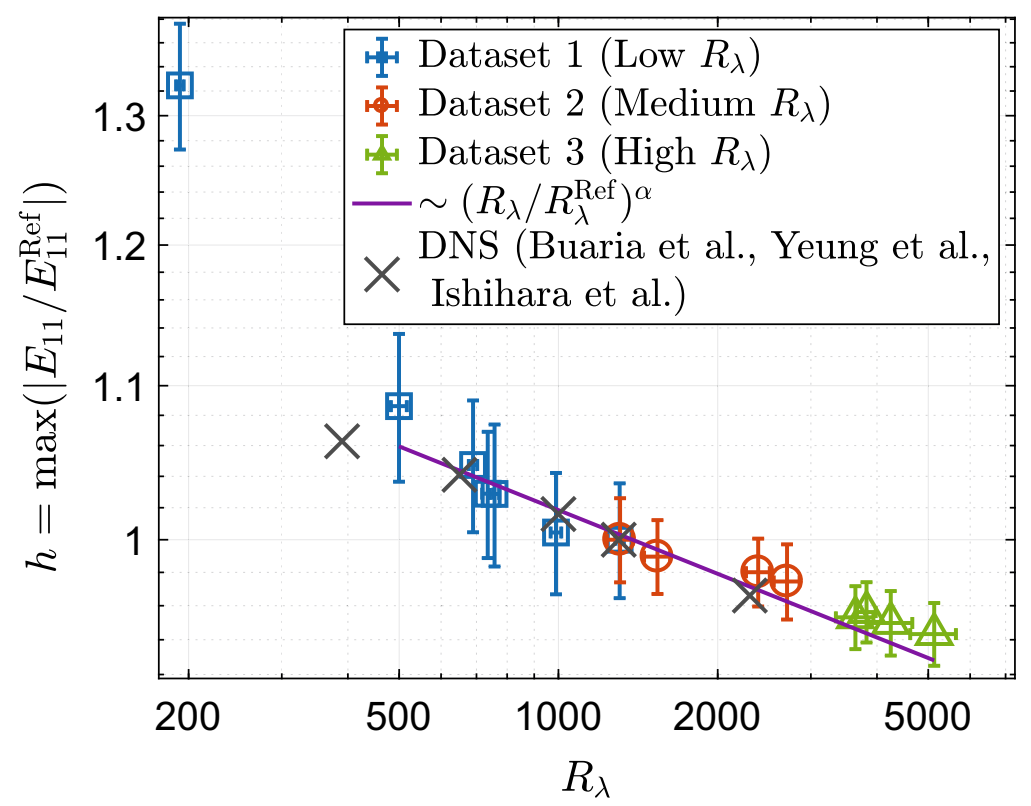

Fig. 7 We have shifted Dataset 3 from Fig. 6 under the assumption of a power law $\sim\left(R_{\lambda} / R_{\lambda}^{\text {Ref }}\right)^{\alpha}$ with $\alpha=-0.061 \pm 0.007$, i.e. the position of Dataset 3 with respect to the other Datasets is constructed artificially from the power law in Fig. 6 to allow us to plot the bottleneck scaling with $R_{\lambda}$ alone, i.e. without dividing by $R_{\lambda}^{\text {Ref }}$. We have no physical justification for this power law and stress that the position of Dataset 3 in this figure is speculative 


\section{Discussion}

In this paper we studied the spectra of a turbulent wind tunnel flow of $R_{\lambda}$ between 193 and 5131. We have used regular hot-wires as well as NSTAPs with a state-of-the-art constant temperature anemometer to record single-point two-time statistics of the turbulent fluctuations, in particular energy spectra. However, such spectra can be heavily influenced by non-ideal frequency responses of the circuit. The frequency response is particularly complicated when operating sub-miniature wires like the NSTAP with a CTA $[45,48]$. A constant current anemometer (CCA) might perform better in this respect, because the frequency reponse is limited only by the thermal lag of the wire and no feedback loop is involved. Still, this comes at the expense of a variable wire temperature and resistance.

In an attempt to interpret CTA data suffering from a non-flat frequency response, we consider energy spectra relative to a reference spectrum. Such an analysis significantly restricts the phenomena that can be observed. The bump in the energy spectrum at the transition from the inertial to the dissipation range can still be identified in the relative spectra as a local extremum beyond $k \eta=0.015$.

To the best of our knowledge, no other wind tunnel achieves $R_{\lambda}>5000$ in a gas. Moreover, we do not know of any other quantitative study of the scaling of the bottleneck effect with $R_{\lambda}$ in a laboratory experiment. We attribute this to the difficulties one faces when interpreting energy spectra from CTA measurements at relatively high frequencies: The spectrum is stronlgy influenced by the CTA circuitry and these influences are hard to quantify or eliminate.

With the aforementioned procedures we are able to extract information about the bottleneck effect from instrument-distorted hot wire spectra. We find indications that the bottleneck effect decreases up to $R_{\lambda} \sim 5000$. We fit a power law of $\left(R_{\lambda} / R_{\lambda}^{\text {Ref }}\right)^{-\alpha}$ with $\alpha=0.061 \pm 0.007$, which is close to the value of $\left(R_{\lambda}\right)^{-0.04}$ found by Donzis and Sreenivasan [19]. Their numerical results are in general in good agreement with our experimental data, lending support to the experiment and data anlysis procedure. Our data equally supports Verma and Donzis [11], who predict that the bottleneck scales as $h \sim 1-\gamma\left(1.5 \log _{2}\left(R_{\lambda}\right)\right)^{2 / 3}$. Revisiting Fig. 6, the data is not inconsistent with different $R_{\lambda} / R_{\lambda}^{\text {Ref }}$-scalings of the relative bottleneck height in the different datasets. We have therefore calculated the scaling of the individual datasets and found that while Dataset 2 and 3 have almost identical scaling exponents $(-0.032 \pm 0.012$, and $-0.034 \pm 0.029$, respectively), Dataset 1 shows a scaling of $-0.1528 \pm 0.012$. When excluding the two lowest $R_{\lambda}$, the scaling exponent becomes $-0.083 \pm 0.024$. This points towards different behaviours at low $R_{\lambda}$, probably due to the effects of a not properly developed inertial range, which contaminates the bottleneck scaling. Such a claim is supported by the slopes of the spectra at $0.001<k \eta<0.015$, which clearly get steeper with increasing $R_{\lambda}$ in Dataset 1 , but change very little in Datasets 2 and 3 , indicating a properly developed inertial range. Interestingly, this effect cannot be seen in DNS, where the bottleneck scaling of $R_{\lambda}^{-0.04}$ can be found at low Reynolds numbers.

We attempt to plot the relative bottleneck height as a function of $R_{\lambda}$ alone. This requires the assumption that the aforementioned power law holds and can be extrapolated. Such an assumption is highly speculative and the results should be considered as such.

We can not quantify the absolute height of the bottleneck bump. Yet, we can argue that if the relative spectra are still changing with $R_{\lambda}$ in the relevant region, the effect has not completely vanished. We can find a systematic decrease of the peak in the relative spectra 
for $R_{\lambda}<3000$. The data for $R_{\lambda}>3000$ in Dataset 3 is inconclusive. A small, decreasing trend can be found, consistent with the power law fit. However, the differences in height are so small compared to the error bars that the claim of a constant bottleneck height at $R_{\lambda}>3000$ would also be supported by the data, especially when considering alternative definitions of the bottleneck height in relative spectra as in Fig. 11 found in the appendix. This is not in contradiction to the atmospheric spectra mentioned above, as they have an even higher $R_{\lambda}$. Further, we note that a bottleneck effect might not show up as a peak in a 5/3-compensated spectrum, yet might be present when compensating by an intermittencycorrected slope $-(5 / 3+\beta)$. In this case, the bottleneck effect would still be visible in the relative spectrum. However, the claim that the bottleneck height does not change with $R_{\lambda}$ for $R_{\lambda}>3000$ is not ruled out by the data.

As far as this study is concerned, the data matches the predictions of Verma and Donzis [11]: The bottleneck height decreases with increasing $R_{\lambda}$, but relatively high $R_{\lambda}$ are necessary to make the effect vanish completely. Based on nonlinear and nonlocal shell-to-shell energy transfer Verma and Donzis [11] estimate that the bottleneck is basically absent for $R_{\lambda}>10^{4}$, but acknowledge that this might be an overestimate. While lending support to existing studies of the bottleneck effect, especially [19] and theories that incorporate a $R_{\lambda}$-dependence of the peak height, an investigation of the effect in terms of absolute measurements of spectra seems necessary to confirm these claims experimentally. With subminiature probes of low thermal lag, such a study might be possible with a constant current anemometer, whose frequency response is intriniscally more simple. However, the present study could reliably measure how the bottleneck decreases with increasing $R_{\lambda}$ for the first time in an laboratory experiment and for $R_{\lambda}$ much higher than achieved in DNS or other wind tunnel studies.

Acknowledgements Open access funding provided by Max Planck Society. The operation of the experiment would be impossible without the help and expertise of A. Kubitzek, A. Kopp, A. Renner, U. Schminke and O. Kurre. The NSTAPs were generously provided by M. Hultmark and Y. Fan. We thank P. K. Yeung, D. Buaria, and T. Ishihara for providing the numerical data. We thank D. Lohse and P. Roche for useful comments.

Open Access This article is distributed under the terms of the Creative Commons Attribution 4.0 International License (http://creativecommons.org/licenses/by/4.0/), which permits unrestricted use, distribution, and reproduction in any medium, provided you give appropriate credit to the original author(s) and the source, provide a link to the Creative Commons license, and indicate if changes were made.

\section{Appendix A: Brief Description of the Wind Tunnel}

The VDTT consists of two $11.7 \mathrm{~m}$ long straight cylindrical tubes connected by two elbows of center-line radius of $1.75 \mathrm{~m}$. The tunnel was filled with sulfur-hexaflouride (SF6) at pressures between 1.5 and 15 bar for the measurements presented here.

The flow is propelled by a fan rotating at up to $24 \mathrm{~Hz}$ creating mean flow speeds of up to $5.5 \mathrm{~m} / \mathrm{s}$. It passes the first elbow and enters a heat exchanger, which removes any turbulent energy dissipated into heat and thus keeps the temperature in the tunnel constant. The rectangular cross-section of the heat exchanger is smoothly adapted to the tunnel's circular geometry by contractions. The vertical slots of the heat exchanger are expected to destroy large-scales structure present in the flow. After the heat exchanger, the flow passes an $80 \mathrm{~cm}$ long expansion, which adapts it to the measurement section. While passing this expansion the flow is stabilized and homogenized by three consecutive meshes of ascending spacing. The flow enters a $9 \mathrm{~m}$ long measurement section through an $104 \mathrm{~cm}$ high active grid, 
which is directly followed by a $70 \mathrm{~cm}$ long expansion to the measurement section's height of $117 \mathrm{~cm}$. The measurement section is followed by another elbow and enters a second measurement section through another sequence of three meshes before being accelerated again by the fan.

\section{Appendix B: Data Acquisition and Analysis Procedure}

The NSTAPs were operated following largely [61] using a Dantec StreamLine 90C10 module within a 90N10 frame. The CTA bridge was set to a 1:1 ratio and the overheat is determined by an external resistor $R_{\text {ext }}$ connected to the system. Typical overheat ratios $R_{\text {ext }} / R_{\text {Probe }}$ were 1.2-1.3, where $R_{\text {Probe }} \sim 100 \Omega$ denotes the probe cold resistance. The Dantec wires were used in a 1:20 bridge utilizing the internal automatics to set the overheat. The data was acquired in the following procedure: The hot wire frequency response and proper operation was tested on a very basic level using the square wave test built into the Dantec CTA-system. The hot-wire system was calibrated by scanning a range of mean flow speeds set by the fan frequency in the tunnel. We determined the mean flow speed through the differential pressure between a pitot tube and a static pressure probe. The differential pressure was picked up by a Siemens SITRANS differential pressure transfucer/ We chose $\sim 20$ calibration points spaced by $\sim 0.1 \mathrm{~m} / \mathrm{s}$. The probe voltage was recorded for $60 \mathrm{~s}$ along with the mean pressure difference, a voltage-velocity curve was calculated, and King's law was fitted to the data. In between calibration points we waited for $45 \mathrm{~s}$ for the mean flow to become stationary. The data was recorded with a National Instruments NI PCI-6123 16-bit DAQ-Card at sampling rates of 60 or $200 \mathrm{kHz}$. Higher sampling rates were used for NSTAP measurements, where the CTA analog low-pass filter was set to $100 \mathrm{kHz}$. When using standard hot wires, the filter frequency was set to $30 \mathrm{kHz}$ and the data was sampled at $60 \mathrm{kHz}$. The data was recorded in segments of 6 million voltage samples, each saved to disk in a 16-bit binary format.

We shall briefly outline the initial data analysis procedure used to obtain essential turbulence statistics as well as the power spectrum. Each of the following steps was carried out on each segment and the results were averaged over all files in the end. We used King's law with parameters obtained from the calibration data to convert the voltages to velocities. Note that the shape of the energy-frequency spectrum is independent of the calibration, which is only required to obtain its absolute value. Because the analog filtering was not sufficient to filter out all noise, we low-pass filtered the data digitally using a butterworthFilter of order 3 in forward and reverse directions. This introduces edge effects, which we remove by cutting the first and last 60 points of the time series. We then subtract the mean $U$ from the velocity time series to obtain a time series of $u$. The remaining analysis is performed on this filtered dataset. The power spectra were calculated using MATLB's fft function, which is based on the FFTW-package. We calculate the correlation function using MATLAB's xcorr function, which itself relies on the aforementioned fourier transform procedure as well as structure functions of order 1 to 8 . Finally, we obtain histograms of velocity and voltage. We use Taylor's Hypothesis, which assumes that a one-dimensional velocity field can be obtained from a time series by multiplying the time increments by the mean velocity: $\Delta x=\Delta t \cdot U$. The power spectra are normalized using the assumption that $\int E(k) d k=u^{2}$.

We routinely calculate basic turbulence quantities in different ways and check the results for consistency. The quantites $R_{\lambda}$, and $\eta$ depend on the mean energy dissipation rate $\varepsilon$, 
which we measure using the third-order structure function $S_{3}(r)=\left\langle(u(x+r)-u(x))^{3}\right\rangle=$ $4 / 5(\varepsilon r)$. The last step follows from the Navier-Stokes equations and is also predicted by Kolmogorov's 1941 theory. In practice we estimate $\varepsilon=\max \left(5 / 4 S_{3} / r\right)$ and check the result with $\varepsilon=15 v \int k^{2} E(k) d k$, and $\varepsilon=\max \left(S_{2}^{3 / 2} / r\right)$. The integral length scale is calculated as $L=\int_{0}^{\infty} C(r) d r$, where $C(r)=\langle u(x+r) u(x)\rangle$ is the velocity auto-correlation function. Its error mainly stems from the ambiguous choice of the upper integration limit, which leads to a relative error about $10 \%$ in $L$.

\section{Appendix C: Calculation and Cross-Check of Relative Spectra}

To obtain relative spectra, the initial spectrum consisting of 3 million points was downsampled to 50000 logarithmically spaced datapoints. To remove the noise from these spectra, we have smoothed them using a fractional octave smoothing algorithm. It multiplies the spectrum at each frequency with a Gaussian centered around the current frequency $f_{i}$ with a width of $\sigma_{i}=\left(f_{i} / n\right) / \pi$, where $n$ determines the smoothing level. Therefore, the smoothing window is larger for higher frequencies. To estimate the noise level in the spectrum and the associated statistical error, we consider the data within $3 \sigma_{i}$ of each frequency. We estimate the standard error as $\delta=\sqrt{\operatorname{Var} / N}$, where $N$ is the number of points considered and Var denotes their variance. Finally, the compensated spectra are calculated as $\psi(k)=E_{11}(k) k^{5 / 3} \varepsilon^{-2 / 3}$, which can be written as $\psi(f)$ by Taylor's Hypothesis. Finally, we divide the $i$-th spectrum in a dataset by the reference spectrum: $\psi_{i}(f) / \psi_{\operatorname{Ref}}(f)$. The result is normalized at $k \eta=0.015$ to remove offsets introduced by uncertainties in $\varepsilon$ and to simplify comparisons.

An important cross-check of the technique is its independence from the choice of reference spectrum. To this end we have calculated the bottleneck effect according to

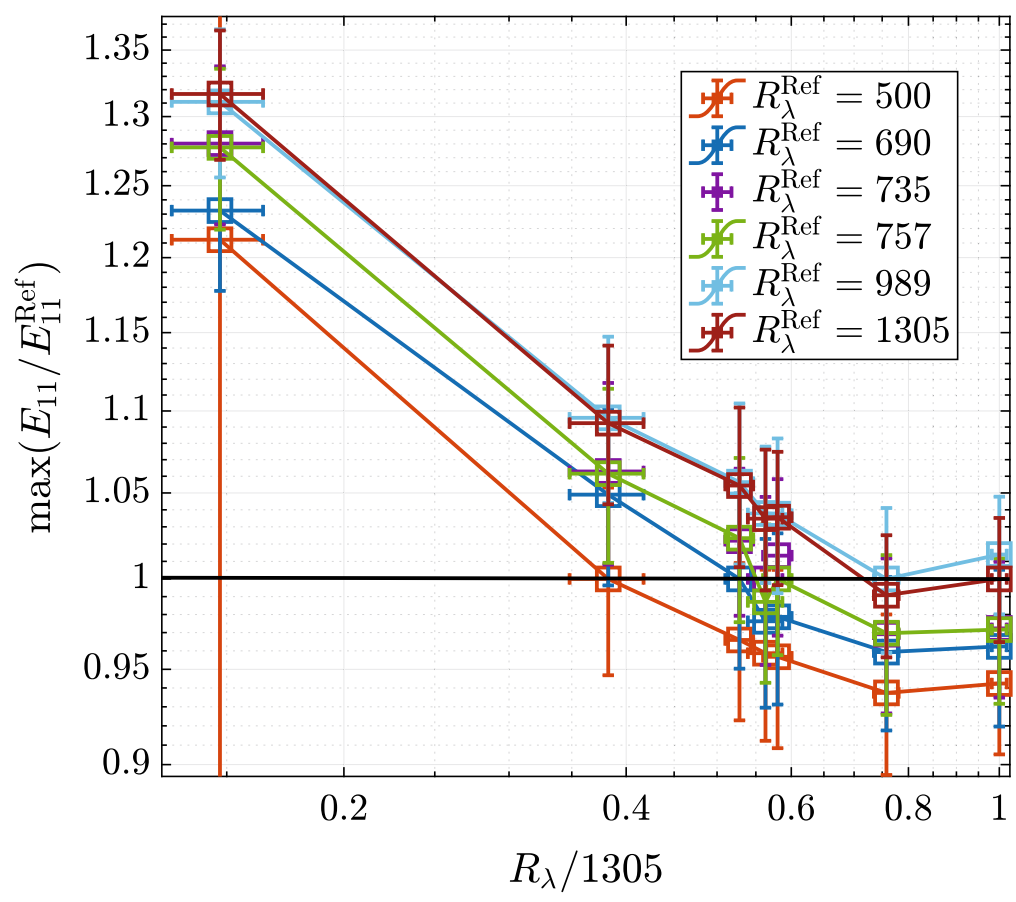

Fig. 8 The bottleneck height in Dataset 1 with different choices of $E_{11}^{\text {Ref }}$. If the analysis is independent of the choice of reference spectrum, the graphs are parallel in this representation. Note that the reference spectrum always has bottleneck height 1 


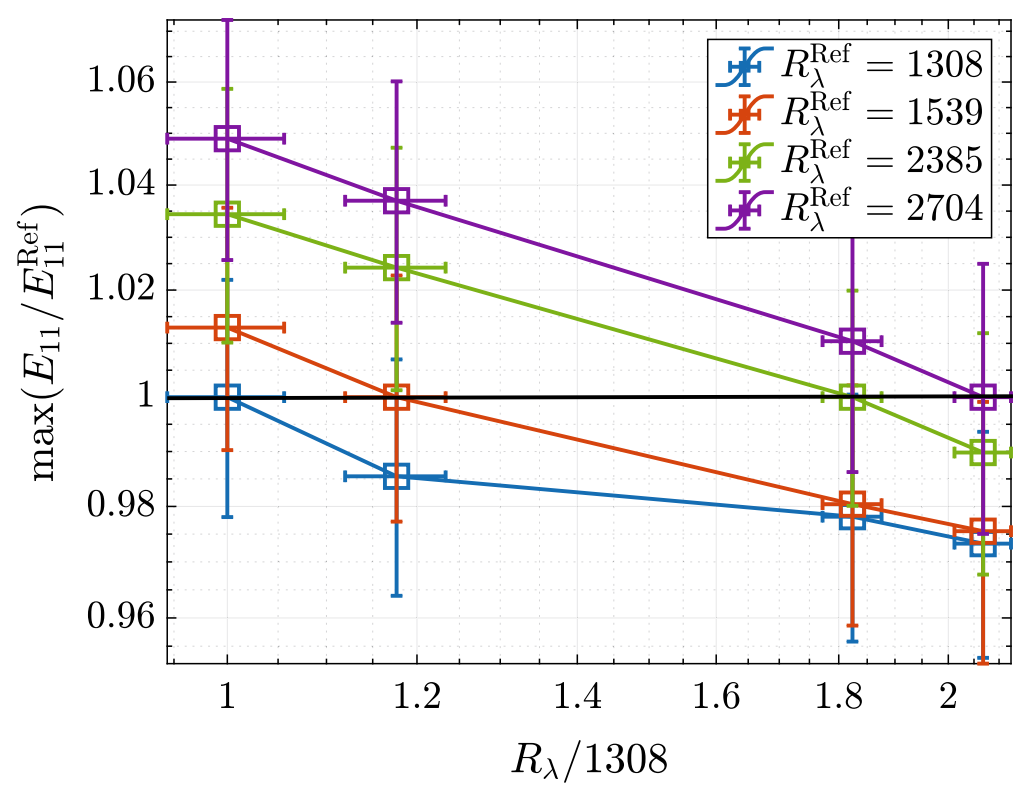

Fig. 9 Same as Fig. 8 for Dataset 2

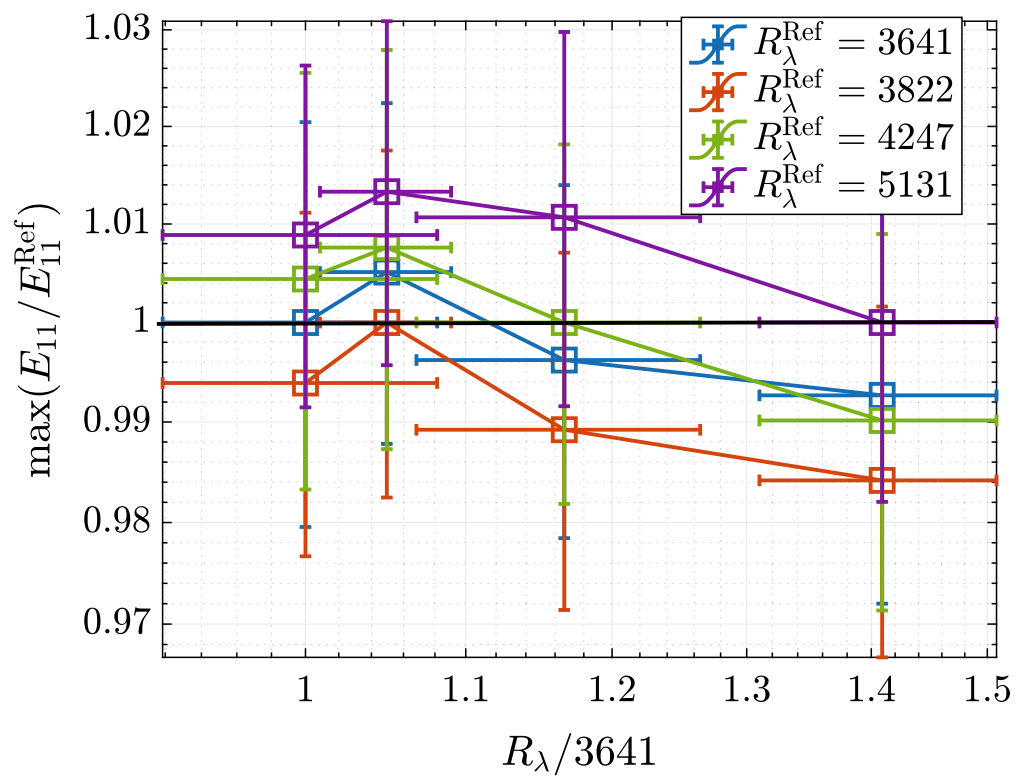

Fig. 10 Same as Fig. 8 for Dataset 3

the analysis outlined above for all possible choices of reference spectra. The results are shown in Figs. 8, 9 and 10. They show the peak height in the relative spectra as a function of $R_{\lambda} / R_{\lambda}^{\mathrm{Ref}} . R_{\lambda} / R_{\lambda}^{\mathrm{Ref}}$ has been normalized to the value that was chosen in the main part of the paper to increase the clarity of the figures. If the analysis is independent of the choice of reference spectrum $E_{11}^{\text {Ref }}$, a different choice $E_{11}^{\prime \text { Ref }}$, should move the resulting curve by a factor of $E_{11}^{\mathrm{Ref}} / E_{11}^{\mathrm{Ref}}$ upwards and $R_{\lambda}^{\mathrm{Ref}} / R_{\lambda}^{\prime \text { Ref }}$ to the right. The latter is trivial and has been removed from Figs. 8, 9 and 10 by the additional normalization. Thus, if the spectra are independent of the choice of reference spectrum, the bottleneck curves should be parallel. Figures 8, 9 and 10 show that this is valid in good approximation showing that the analysis is largely independent of the choice of reference spectrum within a dataset. In a similar way, Fig. 11 shows that the qualitative results are largely independent of where the bottleneck effect is measured. The details are found in Sect. 3.2. 

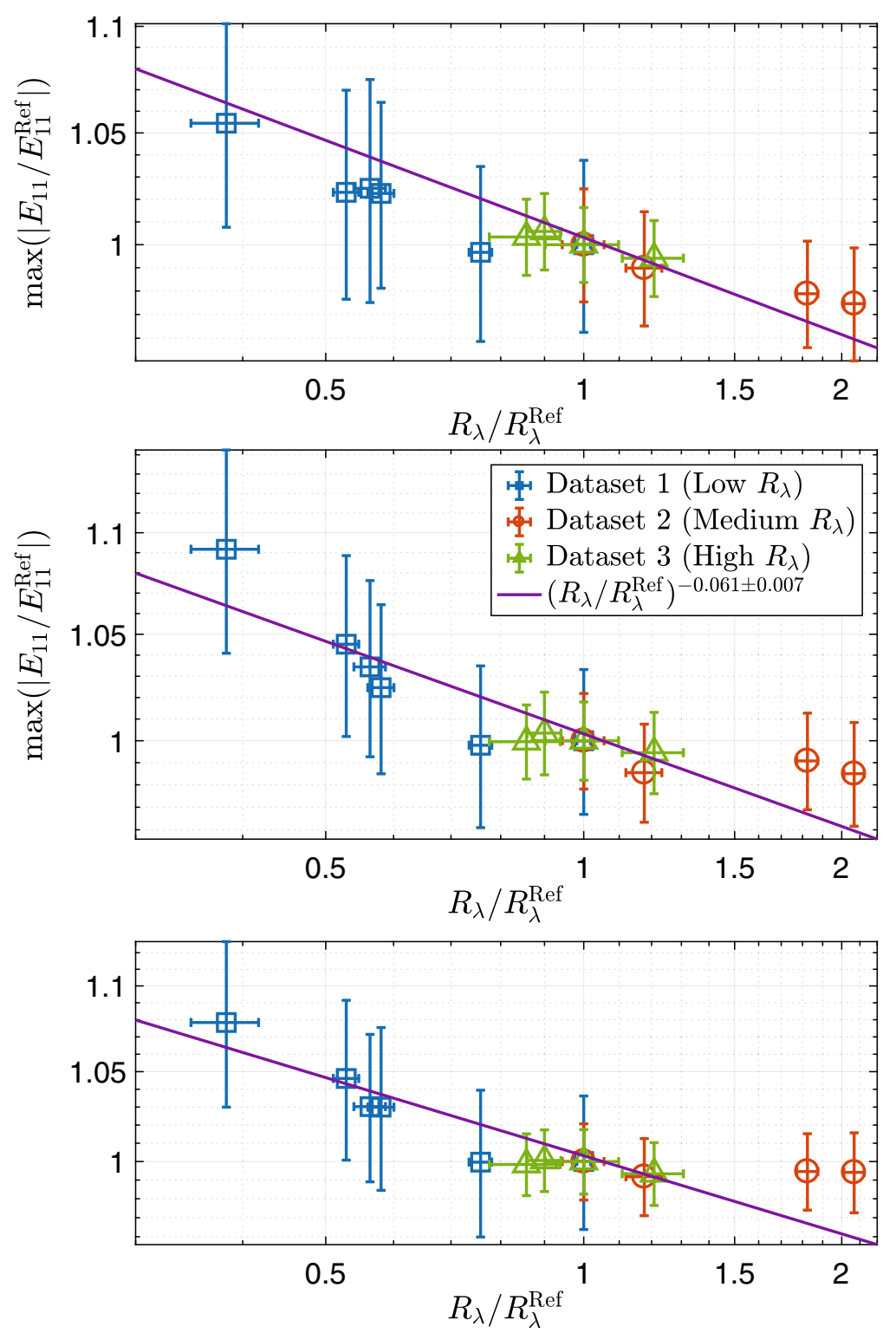

Fig. 11 Bottleneck height as function of $R_{\lambda} / R_{\lambda}^{\text {Ref }}$ with different bottleneck definitions based on the height of the relative spectra at a fixed $k \eta$. The power law is the one found using the initial definition of the bottleneck height in Fig. 6. Upper graph: Bottleneck at $k \eta=0.03$, middle graph at $k \eta=0.04$, lower graph at $k \eta=0.046$ (as predicted by DNS). Datasets 1 and 2 still follow the trend found in the main part. Dataset 3 is not inconsistent with the claim of a constant bottleneck height at $R_{\lambda}>3000$

\section{Appendix D: On the quantity $C_{\varepsilon}=L \varepsilon / u^{3}$}

In Fig. 2c we plot $L / \eta$ as a function of $R_{\lambda}$ and recover to a good approximation the expected scaling of $L / \eta \sim R_{\lambda}^{3 / 2}$. This scaling is derived from the relation $\varepsilon=C_{\varepsilon} u^{3} / L$ and the definitions of $\eta=\left(v^{3} / \varepsilon\right)^{1 / 4}$ and $\lambda=\sqrt{15 v u^{2} / \varepsilon}$. Thus, the scaling depends on the constant $C_{\varepsilon}$, which is flow-dependent (see e.g. [36-38] for a review). We plot this constant as a function of $R_{\lambda}$ in Fig. 12, where we have used $L=\int C(r) d r$. Between $R_{\lambda} 500$ and 2000 the value is approximately constant after being higher at lower $R_{\lambda}$. This behaviour is qualitatively consistent with the findings reported by Sreenivasan [36]. In general, $C_{\varepsilon}$ is scattered around 1. We attribute the scatter to the fact that the exact vaue of $C_{\varepsilon}$ depends on the larg-scale flow structure and is thereby influenced by the forcing mechanism. Considering that the active grid forcing is our main means of fine-tuning $R_{\lambda}$, we expect such a scatter in $C_{\varepsilon}$. 


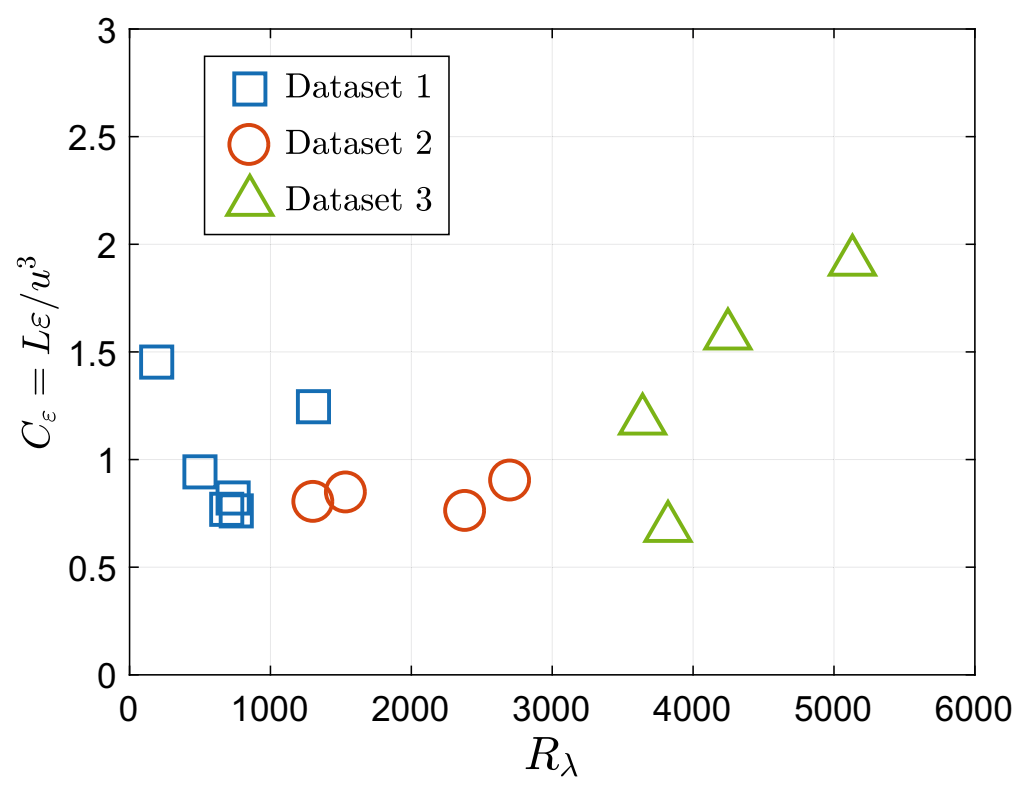

Fig. $12 C_{\varepsilon}=L \varepsilon / u^{3}$ as a function of $R_{\lambda}$. The data is scattered around $C_{\varepsilon}=1$. We attribute the scatter to the dependence of $C_{\varepsilon}$ on the large scale flow structure, which is our main means of changing $R_{\lambda}$

\section{References}

1. Fefferman, C.L.: Existence and smoothness of the navier-stokes equation 57, 67

2. Kolmogorov, A.: The local structure of turbulence in incompressible viscous fluid for very large reynolds numbers. Akademiia Nauk SSSR Doklady 30, 301-305 (1941)

3. Sreenivasan, K.R., Prabhu, A., Narasimha, R.: Zero-crossings in turbulent signals. J. Fluid Mech. 137, 251-272 (1983). https://doi.org/10.1017/S0022112083002396

4. Taylor, G.I.: The spectrum of turbulence. Proc. R. Soc. Lond. A Math. Phys. Sci. 164, 919 (1938). https:// doi.org/10.1098/rspa.1938.0032

5. Saddoughi, S.G., Veeravalli, S.V.: Local isotropy in turbulent boundary layers at high Reynolds number. J. Fluid Mech. 268, 333 (1994). https://doi.org/10.1017/S0022112094001370

6. Mydlarski, L., Warhaft, Z.: On the onset of high-Reynolds-number grid-generated wind tunnel turbulence. J. Fluid Mech. 320, 320-331 (1996). https://doi.org/10.1017/S0022112096007562

7. Verma, M.K., Ayyer, A., Debliquy, O., Kumar, S., Chandra, A.V.: Local shell-to-shell energy transfer via nonlocal interactions in fluid turbulence. Pramana J. Phys. 65(2), 297 (2005). https://doi.org/10.1007/ BF02898618

8. Frisch, U., Kurien, S., Pandit, R., Pauls, W., Ray, S.S., Wirth, A., Zhu, J.Z.: Hyperviscosity, Galerkin truncation, and bottlenecks in turbulence. Phys. Rev. Lett. 101(14), 144501 (2008). https://doi.org/10. 1103/PhysRevLett.101.144501

9. Kurien, S., Taylor, M.A., Matsumoto, T.: Cascade time scales for energy and helicity in homogeneous isotropic turbulence. Phys. Rev. E 69(6), 066313 (2004). https://doi.org/10.1103/PhysRevE.69.066313

10. Falkovich, G.: Bottleneck phenomenon in developed turbulence. Phys. Fluids 6(4), 1411-1414 (1994). https://doi.org/10.1063/1.868255

11. Verma, M.K., Donzis, D.: Energy transfer and bottleneck effect in turbulence. J. Phys. A: Math. Theor. 40(16), 4401-4412 (2007). https://doi.org/10.1088/1751-8113/40/16/010

12. Yakhot, V., Zakharov, V.: Hidden conservation laws in hydrodynamics; energy and dissipation rate fluctuation spectra in strong turbulence. Phys. D: Nonlinear Phenom. 64(4), 379-394 (1993). https://doi.org/ 10.1016/0167-2789(93)90050-B

13. She, Z.S., Jackson, E.: On the universal form of energy spectra in fully developed turbulence. Phys. Fluids A 5(7), 1526-1528 (1993). https://doi.org/10.1063/1.858591

14. Kang, H.S., Chester, S., Meneveau, C.: Decaying turbulence in an active-grid-generated flow and comparisons with large-eddy simulation. J. Fluid Mech. 480, 129-160 (2003). https://doi.org/10.1017/ S0022112002003579

15. Bodenschatz, E., Bewley, G.P., Nobach, H., Sinhuber, M., Xu, H.: Variable density turbulence tunnel facility. Rev. Sci. Instrum. 85(9), 093908 (2014). https://doi.org/10.1063/1.4896138 
16. Khurshid, S., Donzis, D.A., Sreenivasan, K.R.: Energy spectrum in the dissipation range. Phys. Rev. Fluids 3, 8 (2018). https://doi.org/10.1103/PhysRevFluids.3.082601

17. Ishihara, T., Morishita, K., Yokokawa, M., Uno, A., Kaneda, Y.: Energy spectrum in high-resolution direct numerical simulations of turbulence. Phys. Rev. Fluids 1, 8 (2016). https://doi.org/10.1103/ PhysRevFluids. 1.082403

18. Ishihara, T., Kaneda, Y., Yokokawa, M., Itakura, K., Uno, A.: Energy spectrum in the near dissipation range of high resolution direct numerical simulation of turbulence. J. Phys. Soc. Jpn. 74(5), 1464-1471 (2005). https://doi.org/10.1143/JPSJ.74.1464

19. Donzis, D.A., Sreenivasan, K.R.: The best bottleneck effect and the Kolmogorov constant in isotropic turbulence. J. Fluid Mech. 657, 171-188 (2010). https://doi.org/10.1017/S0022112010001400

20. Gulitski, G., Kholmyansky, M., Kinzelbach, W., Lüthi, B., Tsinober, A., Yorish, S.: Velocity and temperature derivatives in high-Reynolds-number turbulent flows in the atmospheric surface layer. Part 1. Facilities, methods and some general results. J. Fluid Mech. 589, 57-81 (2007). https://doi.org/10.1017/ S0022112007007495

21. Sreenivasan, K.R., Dhruva, B.: Is there scaling in high-Reynolds-number turbulence? Prog. Theor. Phys. Suppl. 130, 103-120 (1998). https://doi.org/10.1143/PTPS.130.103

22. Tsuji, Y.: Intermittency effect on energy spectrum in high-reynolds number turbulence. Phys. Fluids 16, L43-L46 (2004). https://doi.org/10.1063/1.1689931

23. Dobler, W., Haugen, N.E.L., Yousef, T.A., Brandenburg, A.: Bottleneck effect in three-dimensional turbulence simulations. Phys. Rev. E 68(2), 026304 (2003). https://doi.org/10.1103/PhysRevE.68.026304

24. Lohse, D., Mueller-Groeling, A.: Bottleneck effects in turbulence: scaling phenomena in r-versus p-space. Phys. Rev. Lett. 74(10), 1747-1750 (1995). https://doi.org/10.1103/PhysRevLett.74.1747

25. Sinhuber, M., Bewley, G.P., Bodenschatz, E.: Dissipative effects on inertial-range statistics at high Reynolds numbers. Phys. Rev. Lett. 119(13), 134502 (2017). https://doi.org/10.1103/PhysRevLett.119. 134502

26. Taylor, G.I.: Statistical theory of turbulence. Proc. R. Soc. Lond. A Math. Phys. Sci. 151(873), 421 (1935). https://doi.org/10.1098/rspa.1935.0158

27. Yeung, P.K., Sreenivasan, K.R., Pope, S.B.: Effects of finite spatial and temporal resolution in direct numerical simulations of incompressible isotropic turbulence. Phys. Rev. Fluids 3(6), 064603 (2018). https://doi.org/10.1103/PhysRevFluids.3.064603

28. Bourgoin, M., Baudet, C., Kharche, S., Mordant, N., Vandenberghe, T., Sumbekova, S., Stelzenmuller, N., Aliseda, A., Gibert, M., Roche, P.E., Volk, R., Barois, T., Caballero, M.L., Chevillard, L., Pinton, J.F., Fiabane, L., Delville, J., Fourment, C., Bouha, A., Danaila, L., Bodenschatz, E., Bewley, G., Sinhuber, M., Segalini, A., Örlü, R., Torrano, I., Mantik, J., Guariglia, D., Uruba, V., Skala, V., Puczylowski, J., Peinke, J.: Investigation of the small-scale statistics of turbulence in the Modane S1MA wind tunnel 9(2), 269-281. https://doi.org/10.1007/s13272-017-0254-3

29. Pietropinto, S., Poulain, C., Baudet, C., Castaing, B., Chabaud, B., Gagne, Y., Hebral, B., Ladam, Y., Lebrun, P., Pirotte, O., Roche, P.: Superconducting instrumentation for high Reynolds turbulence experiments with low temperature gaseous helium. Phys. C 386, 512-516 (2003)

30. Salort, J., Chabaud, B., Leveque, E., Roche, P.E.: Energy cascade and the four-fifths law in superfluid turbulence. Europhys. Lett. 97(3), 34006 (2012). https://doi.org/10.1209/0295-5075/97/34006

31. Rousset, B., Bonnay, P., Diribarne, P., Girard, A., Poncet, J.M., Herbert, E., Salort, J., Baudet, C., Castaing, B., Chevillard, L., Daviaud, F., Dubrulle, B., Gagne, Y., Gibert, M., Hebral, B., Lehner, T., Roche, P.E., Saint-Michel, B., Bon Mardion, M.: Superfluid high Reynolds von Kármán experiment. Rev. Sci. Instrum. 85(10), 103908 (2014). https://doi.org/10.1063/1.4897542

32. Saint-Michel, B., Herbert, E., Salort, J., Baudet, C., Bon Mardion, M., Bonnay, P., Bourgoin, M., Castaing, B., Chevillard, L., Daviaud, F., Diribarne, P., Dubrulle, B., Gagne, Y., Gibert, M., Girard, A., Hébral, B., Lehner, T., Rousset, B.: SHREK collaboration: probing quantum and classical turbulence analogy in von Kármán liquid helium, nitrogen, and water experiments. Phys. Fluids 26(12), 125109 (2014). https://doi. org/10.1063/1.4904378

33. Sinhuber, M., Bodenschatz, E., Bewley, G.P.: Decay of turbulence at high Reynolds numbers. Phys. Rev. Lett. 114, 3 (2015). https://doi.org/10.1103/PhysRevLett.114.034501

34. Hideharu, M.: Realization of a large-scale turbulence field in a small wind tunnel. Fluid Dyn. Res. 8(1), 53-64 (1991). https://doi.org/10.1016/0169-5983(91)90030-M

35. Griffin, K.P., Wei, N.J., Bodenschatz, E., Bewley, G.P.: Control of long-range correlations in turbulence. Exp. Fluids (2019). arXiv preprint arXiv:1809.05126

36. Sreenivasan, K.: On the scaling of the turbulence energy dissipation rate. Phys. Fluids 27(5), 1048-1051 (1984). https://doi.org/10.1063/1.864731

37. Sreenivasan, K.: An update on the scaling of the turbulence energy dissipation rate. Phys. Fluids 10, 2 (1998). https://doi.org/10.1063/1.869575 
38. Vassilicos, C.: Dissipation in turbulent flows annular review of fluid mechanics 47, 1 (2015). https://doi. org/10.1146/annurev-fluid-010814-014637

39. Bewley, G., Chang, K., Bodenschatz, E.: On integral length scales in anisotropic turbulence. Phys. Fluids 24, 6 (2012). https://doi.org/10.1063/1.4726077

40. Comte-Bellot, G.: Hot-wire anemometry 8(1), 209-231. https://doi.org/10.1146/annurev.fl.08.010176. 001233

41. Freymuth, P.: Frequency response and electronic testing for constant-temperature hot-wire anemometers. J. Phys. E: Sci. Instrum. 10(7), 705 (1977). https://doi.org/10.1088/0022-3735/10/7/012

42. Perry, A.E., Morrison, G.L.: A study of the constant-temperature hot-wire anemometer. J. Fluid Mech. 47(3), 577 (1971). https://doi.org/10.1017/S0022112071001241

43. Wood, N.B.: A Method for determination and control of the frequency response of the constanttemperature hot-wire anemometer. J. Fluid Mech. 67(4), 769 (1975). https://doi.org/10.1017/ S0022112075000602

44. Watmuff, J.H.: An Investigation of the constant-temperature hot-wire anemometer. Exp. Therm. Fluid Sci. 11, 117-134 (1995). https://doi.org/10.1016/0894-1777(94)00137-W

45. Samie, M., Watmuff, J.H., Van Buren, T., Hutchins, N., Marusic, I., Hultmark, M., Smits, A.J.: Modelling and operation of sub-miniature constant temperature hot-wire anemometry. Meas. Sci. Technol. 27, 125301 (2016). https://doi.org/10.1088/0957-0233/27/12/125301

46. Bonnet, J.P., de Roquefort, T.A.: Determination and optimization of frequency response of constant temperature hot-wire anemometers in supersonic flows. Rev. Sci. Instrum. 51(2), 234-239 (1980). https:// doi.org/10.1063/1.1136180

47. Weiss, J., Knauss, H., Wagner, S.: Method for the determination of frequency response and signal to noise ratio for constant-temperature hot-wire anemometers. Rev. Sci. Instrum. 72, 1904 (2001). https://doi.org/ $10.1063 / 1.1347970$

48. Hutchins, N., Monty, J.P., Hultmark, M., Smits, A.J.: A direct measure of the frequency response of hot-wire anemometers: temporal resolution issues in wall-bounded turbulence. Exp. Fluids 56, 18 (2015). https://doi.org/10.1007/s00348-014-1856-8

49. Wyngaard, J.C.: Measurement of small-scale turbulence structure with hot wires. J. Phys. E: Sci. Instrum. 1, 1105-1108 (1968). https://doi.org/10.1088/0022-3735/1/11/310

50. Pao, Y.: Structure of turbulent velocity and scalar fields at large wavenumbers. Phys. Fluids 8(6), 10631075 (1965). https://doi.org/10.1063/1.1761356

51. McKeon, B., Comte-Bellot, G., Foss, J., Westerweel, J., Scarano, F., Tropea, C., Meyers, J., Lee, J., Cavone, A., Schodl, R., Koochesfahani, M., Andreopoulos, Y., Dahm, W., Mullin, J., Wallace, J., Vukoslavčević, P., Morris, S., Pardyjak, E., Cuerva, A.: Velocity, Vorticity, and Mach Number. In: C. Tropea, A.L. Yarin, J.F. Foss (eds.) Springer Handbook of Experimental Fluid Mechanics, pp. 215-471. Springer Berlin Heidelberg, Berlin, Heidelberg (2007). https://doi.org/10.1007/978-3-540-30299-5_5

52. Ashok, A., Bailey, S.C.C., Hultmark, M., Smits, A.J.: Hot-wire spatial resolution effects in measurements of grid-generated turbulence. Exp. Fluids 53(6), 1713-1722 (2012). https://doi.org/10.1007/s00348-0121382-5

53. Sadeghi, H., Lavoie, P., Pollard, A.: Effects of finite hot-wire spatial resolution on turbulence statistics and velocity spectra in a round turbulent free jet. Exp. Fluids 59(3), 40 (2018). https://doi.org/10.1007/ s00348-017-2486-8

54. Fan, Y., Arwatz, G., Van Buren, T.W., Hoffman, D.E., Hultmark, M.: Nanoscale sensing devices for turbulence measurements. Exp. Fluids 56, 138 (2015). https://doi.org/10.1007/s00348-015-2000-0

55. Kunkel, G., Arnold, C., Smits, A.: Development of NSTAP: Nanoscale Thermal Anemometry Probe. American Institute of Aeronautics and Astronautics (2006). https://doi.org/10.2514/6.2006-3718

56. Bailey, S.C.C., Kunkel, G.J., Hultmark, M., Vallikivi, M., Hill, J.P., Meyer, K.A., Tsay, C., Arnold, C.B., Smits, A.J.: Turbulence measurements using a nanoscale thermal anemometry probe. J. Fluid Mech. 663, 160-179 (2010). https://doi.org/10.1017/S0022112010003447

57. Vallikivi, M., Smits, A.J.: Fabrication and characterization of a novel nanoscale thermal anemometry probe. J. Microelectromech. Syst. 23(4), 899-907 (2014). https://doi.org/10.1109/JMEMS.2014.2299276

58. Buaria, D., Sawford, B.L., Yeung, P.K.: Characteristics of backward and forward two-particle relative dispersion in turbulence at different Reynolds numbers. Phys. Fluids 27(10), 105101 (2015). https://doi. org/10.1063/1.4931602

59. Yeung, P.K., Zhai, X.M., Sreenivasan, K.R.: Extreme events in computational turbulence. Proc Natl Acad Sci USA 112(41), 12633 (2015). https://doi.org/10.1073/pnas.1517368112

60. Buaria, D., Pumir, A., Bodenschatz, E., Yeung, P.K.: Extreme velocity gradients in turbulent flows. under Review 
61. Fan, Y.: High Resolution Instrumentation for Flow Measurements. Princeton University, Princeton, Thesis (2017)

Publisher's Note Springer Nature remains neutral with regard to jurisdictional claims in published maps and institutional affiliations. 


\section{Bibliography}

[1] C. Tropea, A. L. Yarin, and J. F. Foss, eds. Springer Handbook of Experimental Fluid Mechanics. Berlin, Heidelberg: Springer Berlin Heidelberg, 2007. DOI: 10 . 1007/978-3-540-30299-5.

[2] G. Comte-Bellot. "Hot-wire anemometry". In: Handb. Fluid Dyn. Second Ed. 2016.

[3] Y. Borisenkov et al. "Micro-machined super-miniature hot-film multiarray probe for field experiments with sub-Kolmogorov resolution". In: J. Turbul. 16.6 (2015), pp. 525-539. DOI: 10 . $1080 / 14685248$. 2015. 1014558.

[4] M. Hultmark, A. Ashok, and A. J. Smits. "A new criterion for endconduction effects in hot-wire anemometry". In: Meas. Sci. Technol. 22.5 (May 2011), p. 055401. DOI: 10.1088/0957-0233/22/5/055401.

[5] M. Vallikivi et al. "Turbulence measurements in pipe flow using a nano-scale thermal anemometry probe". In: Exp. Fluids 51.6 (Dec. 2011), pp. 1521-1527. DOI: $10.1007 /$ s00348-011-1165-4.

[6] S. C. C. Bailey et al. "Turbulence measurements using a nanoscale thermal anemometry probe". In: J. Fluid Mech. 663 (2010), pp. 160-179. Dor: 10.1017/S0022112010003447.

[7] Y. Fan et al. "Nanoscale sensing devices for turbulence measurements". In: Exp. Fluids 56.7 (2015). DOI: 10.1007/s00348-015-2000-0.

[8] H. Le-The et al. "Fabrication of free-standing Pt nanowires used as thermal anemometry probes for turbulence measurements". In: Nat. Microsystems Nanoeng. under consideration (2020).

[9] S. C. C. BAILEY et al. "Turbulence measurements using a nanoscale thermal anemometry probe". In: J. Fluid Mech. 663 (Nov. 2010), pp. 16o179. DOI: $10.1017 / \mathrm{S} 0022112010003447$.

[10] M. Samie et al. "Modelling and operation of sub-miniature constant temperature hot-wire anemometry". In: Meas. Sci. Technol. 27.12 (2016). DOI: $10.1088 / 0957-0233 / 27 / 12 / 125301$.

[11] M. Samie et al. "Fully resolved measurements of turbulent boundary layer flows up to". In: J. Fluid Mech. 851 (Sept. 2018), pp. 391-415. Dor: 10.1017/jfm.2018.508. 
[12] M. Samie, N. Hutchins, and I. Marusic. "Revisiting end conduction effects in constant temperature hot-wire anemometry". In: Exp. Fluids 59.9 (Sept. 2018), p. 133. DOI: 10.1007/s00348-018-2587-z.

[13] H. Sadeghi, P. Lavoie, and A. Pollard. "Effects of finite hot-wire spatial resolution on turbulence statistics and velocity spectra in a round turbulent free jet". In: Exp. Fluids 59.3 (2018), pp. 1-10. DOI: $10.1007 /$ s00348-017-2486-8.

[14] M. K. Fu, Y. Fan, and M. Hultmark. "Design and validation of a nanoscale cross-wire probe (X-NSTAP)". In: Exp. Fluids 60.6 (June 2019), p. 99. DOI: $10.1007 / \mathrm{s} 00348-019-2743-0$.

[15] G. Kunkel, C. Arnold, and A. Smits. “Development of NSTAP: nanoscale thermal anemometry probe". In: 36th AIAA Fluid Dyn. Conf. June (2006), pp. 1-8.

[16] V. Emsellem et al. "Transitions and probes in turbulent helium". In: Phys. Rev. E 55.3 (Mar. 1997), pp. 2672-2681. DOI: 10.1103/PhysRevE. 55.2672.

[17] G. Zocchi et al. "Mesurement of the scaling of the dissipation at high Reynolds numbers". In: Phys. Rev. E 50.5 (1994), pp. 3693-3700. DOI: 10.1103/PhysRevE.50.3693.

[18] S. Grossmann and D. Lohse. "Characteristic scales in Rayleigh-Benard turbulence". In: Phys. Lett. A 173.1 (Jan. 1993), pp. 58-62. DoI: 10.1016/ 0375-9601 (93) 90087-G.

[19] D. Bestion, J. Gaviglio, and J. P. Bonnet. "Comparison between constantcurrent and constant-temperature hot-wire anemometers in high-speed flows". In: Rev. Sci. Instrum. 54.11 (Nov. 1983), pp. 1513-1524. DOI: 10 . 1063/1.1137279.

[20] J. H. Watmuff. "An investigation of the constant-temperature hot-wire anemometer". In: Exp. Therm. Fluid Sci. 11.2 (Aug. 1995), pp. 117-134. DOI: $10.1016 / 0894-1777$ (94)00137-W.

[21] A. E. Perry and G. L. Morrison. "A study of the constant-temperature hot-wire anemometer". In: J. Fluid Mech. (1971). DOI: 10.1017/S0022112071001241.

[22] G. Comte-Bellot and S. Corrsin. "Simple Eulerian time correlation of full-and narrow-band velocity signals in grid-generated, 'isotropic' turbulence". In: J. Fluid Mech. (1971). DOI: 10.1017/S0022112071001599. 
[23] N. Hutchins et al. "A direct measure of the frequency response of hotwire anemometers: temporal resolution issues in wall-bounded turbulence". In: Exp. Fluids 56.1 (2015). DOI: 10.1007/s00348-014-1856-8.

[24] J. Weiss, H. Knauss, and S. Wagner. "Method for the determination of frequency response and signal to noise ratio for constant-temperature hot-wire anemometers". In: Rev. Sci. Instrum. 72.3 (2001), pp. 19041909. DOI: $10.1063 / 1.1347970$.

[25] J. P. Bonnet and T. Alziary de Roquefort. "Determination and optimization of frequency response of constant temperature hot-wire anemometers in supersonic flows". In: Rev. Sci. Instrum. 51.2 (Feb. 1980), pp. 234239. DOI: $10.1063 / 1.1136180$.

[26] A. J. Smits, A. E. Perry, and P. H. Hoffmann. "The response to temperature fluctuations of a constant-current hot-wire anemometer". In: J. Phys. E. 11.9 (Sept. 1978), pp. 909-914. DOI: 10.1088/0022-3735/11/ 9/012.

[27] G. I. Taylor. "The Spectrum of Turbulence". In: Proc. R. Soc. A Math. Phys. Eng. Sci. 164.919 (Feb. 1938), pp. 476-490. Dor: 10 .1098/rspa . 1938.0032.

[28] R. H. Kraichnan. "Kolmogorov's Hypotheses and Eulerian Turbulence Theory". In: Phys. Fluids (1964). DOI: 10.1063/1.2746572.

[29] H. Tennekes. "Eulerian and Lagrangian time microscales in isotropic turbulence". In: J. Fluid Mech. (1975). DOI: 10.1017/S0022112075000468.

[30] M. Wilczek and Y. Narita. "Wave-number-frequency spectrum for turbulence from a random sweeping hypothesis with mean flow". In: Phys. Rev. E 86.6 (Dec. 2012), p. 066308. DOI: 10.1103/PhysRevE. 86. 066308.

[31] P. Sagaut and C. Cambon. Homogeneous turbulence dynamics. 2018. DoI: 10.1007/978-3-319-73162-9.

[32] S. B. Pope. "Turbulent Flows". In: Cambridge Univ. Press. Vol. 1. 2000. Chap. 6 - Scalin, pp. 232-233. DOI: 10.1088/1468-5248/1/1/702.

[33] A. S. Monin, A. M. Yaglom, and T. S. Lundgren. "Statistical Fluid Mechanics, Vol. II". In: J. Appl. Mech. $43 \cdot 3$ (Sept. 1976), pp. 521-521. DOI: 10.1115/1.3423915.

[34] M. Wilczek, H. Xu, and Y. Narita. "A note on Taylor's hypothesis under large-scale flow variation". In: Nonlinear Process. Geophys. 21.3 (2014), pp. 645-649. DOI: 10.5194/npg-21-645-2014. 
[35] J. Meyers and C. Meneveau. "A functional form for the energy spectrum parametrizing bottleneck and intermittency effects". In: Phys. Fluids 20.6 (2008). DOI: 10.1063/1.2936312.

[36] G. K. Batchelor. "Pressure fluctuations in isotropic turbulence". In: Math. Proc. Cambridge Philos. Soc. 47.2 (1951), pp. 359-374. DOI: 10 . 1017/S0305004100026712.

[37] D. Lohse and A. Muller-Groeling. "Bottleneck effects in turbulence: Scaling phenomena in r versus p space". In: Phys. Rev. Lett. 74.10 (1995), pp. 1747-1750. DOI: 10.1103/PhysRevLett.74.1747.

[38] Y. Fan. NSTAP Operation Manual with Dantec Streamline System. Tech. rep. Princeton University, 2016.

[39] M. Frigo and S. Johnson. “The Design and Implementation of FFTW3”. In: Proc. IEEE 93.2 (2005), pp. 216-231.

[40] N. Wiener. "Generalized harmonic analysis". In: Acta Math. (1930). DoI: 10.1007/BF02546511.

[41] A. Khintchine. "Korrelationstheorie der stationären stochastischen Prozesse". In: Math. Ann. 109.1 (Dec. 1934), pp. 604-615. DoI: 10.1007/BF01449156.

[42] A. Kolmogorov. "The Local Structure of Turbulence in Incompressible Viscous Fluid for Very Large Reynolds' Numbers". In: Dokl. Akad. Nauk SSSR 30 (1941), pp. 301-305.

[43] R. A. Antonia et al. "Approach to the 4/5 law in homogeneous isotropic turbulence". In: J. Fluid Mech. 550.1 (2006), pp. 175-184. DoI: 10.1017/ S0022112005008438.

[44] J. C. Wyngaard and H. Tennekes. "Measurements of the Small-Scale Structure of Turbulence at Moderate Reynolds Numbers". In: Cit. Phys. Fluids 13 (1970), p. 1962. DOI: 10.1063/1.1693192.

[45] J. C. Wyngaard. "Measurement of small-scale turbulence structure with hot wires". In: J. Phys. E. 1.11 (Nov. 1968), p. 310. DOI: 10.1088/0022$3735 / 1 / 11 / 310$.

[46] A. Ashok et al. "Hot-wire spatial resolution effects in measurements of grid-generated turbulence". In: Exp. Fluids 53.6 (Dec. 2012), pp. 17131722. DOI: $10.1007 / \mathrm{s} 00348-012-1382-5$.

[47] C. Küchler, E. Bodenschatz, and G. P. Bewley. Scaling in Decaying Turbulence at High Reynolds Numbers. June 2020. 
[48] J. D. Gibbon. "The three-dimensional Euler equations: Where do we stand?" In: Phys. D Nonlinear Phenom. (2008). Dor: 10.1016/j . physd. 2007.10 .014$.

[49] H. K. Moffatt. "Singularities in fluid mechanics". In: Phys. Rev. Fluids (2019). DOI: 10.1103/PhysRevFluids.4.110502.

[50] M. Sinhuber. "On the Scales of Turbulent Motion at High Reynolds Numbers". PhD thesis. Georg-August-Universität Göttingen, 2015.

[51] P.-F. Yang, A. Pumir, and H. Xu. “Generalized self-similar spectrum and the effect of large-scale in decaying homogeneous isotropic turbulence". In: New J. Phys. 20.10 (Oct. 2018), p. 103035. DOI: 10.1088/13672630/aae72d.

[52] K. P. Griffin et al. "Control of long-range correlations in turbulence". In: Exp. Fluids 6o.4 (Apr. 2019), p. 55. DOI: 10.1007/s00348-019-26981.

[53] D. O. Mora et al. "Energy cascades in active-grid-generated turbulent flows". In: (Mar. 2019). DOI: 10.1103/PhysRevFluids .4.104601.

[54] B. R. Pearson, P.- $\AA$. Krogstad, and W. van de Water. "Measurements of the turbulent energy dissipation rate". In: Phys. Fluids 14.3 (Mar. 2002), pp. 1288-1290. DOI: 10.1063/1.1445422.

[55] S. Risius et al. "Schneefernerhaus as a mountain research station for clouds and turbulence". In: Atmos. Meas. Tech. 8.8 (Aug. 2015), pp. 32093218. DOI: $10.5194 /$ amt-8-3209-2015.

[56] B. Saint-Michel et al. "Probing quantum and classical turbulence analogy in von Kármán liquid helium, nitrogen, and water experiments". In: Phys. Fluids (2014). DoI: 10.1063/1.4904378.

[57] K. R. Sreenivasan. "On the scaling of the turbulence energy dissipation rate". In: Phys. Fluids 27 (1984), p. 528. Dor: 10.1063/1.864731.

[58] K. R. Sreenivasan. "An update on the energy dissipation rate in isotropic turbulence". In: Phys. Fluids 10.2 (Feb. 1998), pp. 528-529. DOI: 10 . 1063/1.869575.

[59] J. C. Vassilicos. "Dissipation in Turbulent Flows". In: Annu. Rev. Fluid Mech. 47.1 (Jan. 2015), pp. 95-114. DOI: 10 . $1146 /$ annurev-fluid $010814-014637$. 
[6o] P. Burattini, P. Lavoie, and R. A. Antonia. “On the normalized turbulent energy dissipation rate". In: Phys. Fluids 17.9 (2005), pp. 1-4. DoI: 10 . 1063/1.2055529.

[61] A. J. Puga and J. C. LaRue. "Normalized dissipation rate in a moderate Taylor Reynolds number flow". In: J. Fluid Mech. 818 (May 2017), pp. 184-204. DOI: 10.1017/jfm. 2017.47.

[62] W. J. T. Bos, L. Shao, and J.-P. Bertoglio. "Spectral imbalance and the normalized dissipation rate of turbulence". In: Phys. Fluids 19.4 (Apr. 2007), p. 045101. DOI: 10.1063/1.2714079.

[63] L. Mydlarski and Z. Warhaft. "On the onset of high-Reynolds-number grid-generated wind tunnel turbulence". In: J. Fluid Mech. 320.-1 (Aug. 1996), p. 331. DOI: 10.1017/S0022112096007562.

[64] A. Thormann and C. Meneveau. "Decay of homogeneous, nearly isotropic turbulence behind active fractal grids". In: Phys. Fluids 26.2 (Feb. 2014), p. 025112. DOI: $10.1063 / 1.4865232$.

[65] J. Qian. "Slow decay of the finite Reynolds number effect of turbulence". In: Phys. Rev. E - Stat. Physics, Plasmas, Fluids, Relat. Interdiscip. Top. (1999). DOI: 10.1103/PhysRevE.60.3409.

[66] T. S. Lundgren. "Kolmogorov turbulence by matched asymptotic expansions". In: Phys. Fluids 15.4 (2003), pp. 1074-1081. DOI: 10.1063/1. 1558332.

[67] P. Davidson. Turbulence - An Introduction For Scientists and Engineers. Oxford University Press, 2015.

[68] R. A. Antonia et al. "Finite Reynolds number effect and the 4/5 law". In: Phys. Rev. Fluids 4 (2019). DOI: 10.1103/PhysRevFluids .4.084602.

[69] U. Frisch. Turbulence. Cambridge University Press, Nov. 1995. DOI: 10. $1017 /$ CB09781139170666.

[70] D. A. Donzis and K. R. Sreenivasan. "The bottleneck effect and the Kolmogorov constant in isotropic turbulence". In: J. Fluid Mech. 657 (2010), pp. 171-188. DOI: 10.1017/S0022112010001400.

[71] K. R. Sreenivasan. "The energy dissipation in turbulent shear flows". In: Symp. Dev. Fluid Dyn. Aerosp. Eng. Bangalore, 1995, pp. 159-190.

[72] A. Praskovsky and S. Oncley. "Measurements of the Kolmogorov constant and intermittency exponent at very high Reynolds numbers". In: Phys. Fluids 6.17 (1994), pp. 1048-2886. DOI: 10.1063/1.2055529. 
[73] R. Benzi et al. "Extended self-similarity in turbulent flows". In: Phys. Rev. E 48.1 (1993), pp. 29-32. DOI: 10.1103/PhysRevE.48.R29.

[74] R. Benzi et al. "On the scaling of three-dimensional homogeneous and isotropic turbulence". In: Phys. D Nonlinear Phenom. 80.4 (Feb. 1995), pp. 385-398. DOI: 10.1016/0167-2789(94)00190-2.

[75] S. Grossmann, D. Lohse, and A. Reeh. "Application of extended selfsimilarity in turbulence". In: Phys. Rev. E - Stat. Physics, Plasmas, Fluids, Relat. Interdiscip. Top. 56.5 (1997), pp. 5473-5478. DoI: 10.1103 / PhysRevE.56.5473.

[76] A. N. Kolmogorov. "A refinement of previous hypotheses concerning the local structure of turbulence in a viscous incompressible fluid at high Reynolds number". In: J. Fluid Mech. 13.1 (May 1962), pp. 82-85. DOI: $10.1017 /$ S0022112062000518.

[77] A. Praskovsky and S. Oncley. "Comprehensive measurements of the intermittency exponent in high reynolds number turbulent flows". In: Fluid Dyn. Res. 21.5 (1997), pp. 331-358. Dor: 10.1016/S0169-5983 (97) 86593-8.

[78] B. Dubrulle. "Intermittency in fully developed turbulence: Log-poisson statistics and generalized scale covariance". In: Phys. Rev. Lett. (1994). DOI: 10.1103/PhysRevLett.73.959.

[79] T. Ishihara. "Energy spectrum in high-resolution direct numerical". In: o82403 (2016), pp. 1-9. DOI: 10.1103/PhysRevFluids.1.082403.

[8o] P. K. Yeung, K. R. Sreenivasan, and S. B. Pope. “Effects of finite spatial and temporal resolution in direct numerical simulations of incompressible isotropic turbulence". In: Phys. Rev. Fluids 3.6 (2018), pp. 115. DOI: 10.1103/PhysRevFluids.3.064603.

[81] M. Sinhuber, G. P. Bewley, and E. Bodenschatz. "Dissipative Effects on Inertial-Range Statistics at High Reynolds numbers". In: Phys. Rev. Lett. 119.1962 (Sept. 2016), p. 134502. DOI: 10.1103/PhysRevLett.119. 134502.

[82] K. P. Iyer et al. "Oscillations modulating power law exponents in isotropic turbulence: Where experiments and simulations agree and differ". In: (Apr. 2020), pp. 1-5.

[83] C. Küchler. "First Realization of ultra-high Reynolds Numbers in the Variable Density Turbulence Tunnel". M.Sc. Thesis. Georg-August-University, Göttingen, 2017. 
[84] E. A. Novikov. "The effects of intermittency on statistical characteristics of turbulence and scale similarity of breakdown coefficients". In: Phys. Fluids A Fluid Dyn. 2.5 (May 1990), pp. 814-820. DoI: 10.1063/1. 857629.

[85] E. A. Novikov. "Infinitely divisible distributions in turbulence". In: Phys. Rev. E 50.5 (1994), pp. 3303-3305. DOI: $10.1103 /$ PhysRevE. 50. R3303.

[86] E. A. Novikov. "Mathematical model for the intermittence of turbulent flow". In: Dokl. Akad. Nauk SSSR 168.6 (1966), pp. 1279-1282.

[87] W.-X. Zhou and D. Sornette. "Evidence of intermittent cascades from discrete hierarchical dissipation in turbulence". In: Phys. D Nonlinear Phenom. 165.1-2 (May 2002), pp. 94-125. DOI: 10.1016/S0167-2789 (02) $00390-1$.

[88] F. Anselmet et al. "High-order velocity structure functions in turbulent shear flows". In: J. Fluid Mech. 140 (Mar. 1984), pp. 63-89. DoI: 10 . 1017/S0022112084000513.

[89] R. Benzi et al. "Related content A Prediction of the Multifractal Model : the Intermediate Dissipation Range A Prediction of the Multifractal Model : the Intermediate Dissipation Range ." In: (1991).

[9o] K. Konno and T. Ohmachi. “Ground-motion characteristics estimated from spectral ratio between horizontal and vertical components of microtremor". In: Bull. Seismol. Soc. Am. 88.1 (1998), pp. 228-241.

[91] M. Beyreuther et al. "ObsPy: A Python Toolbox for Seismology". In: Seismol. Res. Lett. 81.3 (May 2010), pp. 530-533. DOI: 10.1785/gssrl. 81.3 .530$.

[92] C. Meneveau and K. R. Sreenivasan. "Simple multifractal cascade model for fully developed turbulence". In: Phys. Rev. Lett. 59.13 (Sept. 1987), pp. 1424-1427. DOI: 10.1103/PhysRevLett.59.1424.

[93] W.-X. Zhou and D. Sornette. "Evidence of intermittent cascades from discrete hierarchical dissipation in turbulence". en. In: Phys. D Nonlinear Phenom. 165.1-2 (May 2002), pp. 94-125. DOI: 10.1016/S01672789 (02) 00390-1.

[94] C. Küchler, G. Bewley, and E. Bodenschatz. "Experimental Study of the Bottleneck in Fully Developed Turbulence". In: J. Stat. Phys. 175.3-4 (May 2019), pp. 617-639. DOI: 10.1007/s10955-019-02251-1. 


\section{Part III}

\section{Towards Lagrangian Particle Tracking}





\section{Chapter 10}

\section{Lagrangian Particle Tracking in the Variable Density Turbulence}

Tunnel

This chapter introduces the current implementation of Lagrangian Particle Tracking in the Variable Density Turbulence Tunnel. It is structured as follows: First, the design considerations necessary to realise the tracking of particles moving in a extremely turbulent flow inside a $\mathrm{SF}_{6}$ filled pressure vessel are introduced. The current camera technology is compared to the turbulent time scales to be expected, and the resulting requirements on the tracer particles are discussed. Finally, the necessary illumination is assessed. Then, the realisation of Lagrangian particle tracking in the VDTT is detailed. This rather technical section will introduce the particles chosen as well as their dispersion mechanism, then the imaging setup will be described and finally, the illumination system is presented.

\subsection{Design Considerations}

\subsubsection{Turbulent Length- and Time Scales}

The central turbulence quantity for the assessment of a Lagrangian particle tracking system is the Kolmogorov time $\tau_{\eta}=\sqrt{v / \varepsilon}$. Fig. 10.1 shows how $\tau_{\eta}$ behaves in the VDTT flow. Since the kinematic viscosity scales with $1 / p$, a higher facility pressure leads to a smaller $\tau_{\eta}$. Equally, a larger turbulence dissipation rate $\varepsilon$ yields a smaller $\tau_{\eta}$. At a fixed facility pressure, $\varepsilon$ is determined by the ratio $u^{3} / L$. Thus, $\tau_{\eta} \sim \sqrt{L / u^{3}}$. In an experiment with a strong mean flow, such as a wind tunnel, the time- and length scales are closely 


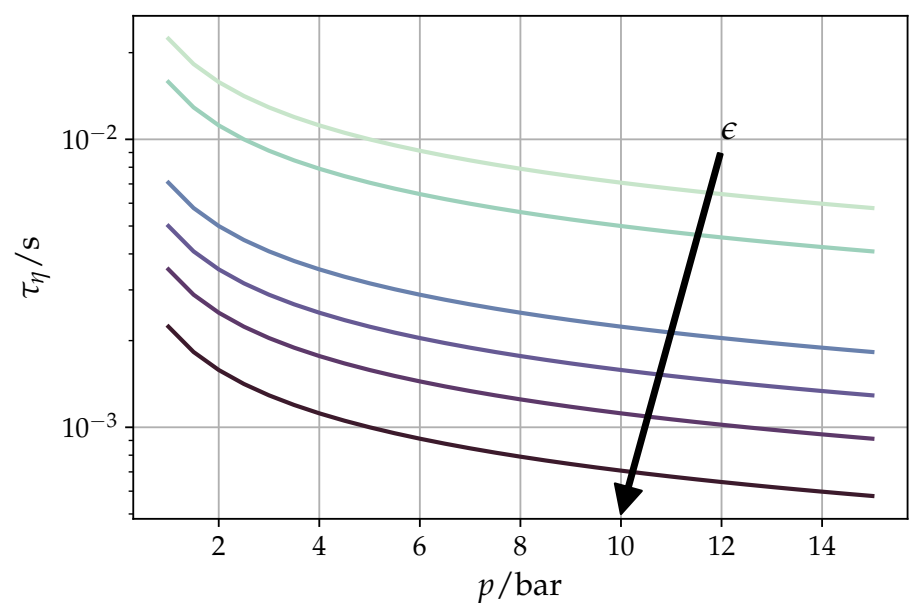

Figure 10.1: $\tau_{\eta}$ as a function of ambient $\mathrm{SF}_{6}$ pressure for $\varepsilon=0.005,0.01,0.05,0.1,0.2,0.5$.

connected. For example, the size of a measurement volume in the direction of the mean flow ultimately determines the number of time scales that can be observed while a particle passes this volume. Fig. 10.1 shows that given a measurement volume, the number of $\tau_{\eta}$ can be adjusted quite freely by changing the facility pressure, the turbulence intensity, the active grid forcing, and the mean flow speed.

The most critical turbulence length scale is the Kolmogorov scale $\eta$, which predominantly depends on $v$ and is therefore largely determined by the facility pressure. The experiment therefore has to be designed to meet the most demanding case of $\eta=10^{-5} \mathrm{~m}$.

\subsubsection{Particles}

The purpose of this setup is to study the motion of tracer particles, i.e. particles that very closely follow the flow without influencing it significantly. The relevant characterisation is therefore the particle response time to a given change in flow [1]

$$
\tau_{P}=\frac{2}{9} a^{2} \frac{\rho_{P}-\rho_{f}}{\mu} .
$$

Here, $a$ is the particle radius, $\mu$ is the dynamic viscosity, and $\rho_{p}$, and $\rho_{f}$ are the particle and fluid density, respectively. Setting this property in relation to the Kolmogorov time yields the Stokes number

$$
\mathrm{St}=\frac{\tau_{P}}{\tau_{\eta}} .
$$


The smaller this number, the closer a particle follows the flow and the smaller are inertial effects. If $\mathrm{St}<0.1$, a particle can be considered a tracer $[1,2,3,4]$.

Therefore, small particles with a density close to that of $\mathrm{SF}_{6}$ are preferred in terms of inertial effects, but require stronger illumination and magnification to record on video. Further, even if density matched, the particles should not be much larger than the Kolmogorov length scale, i.e. $a \sim 10^{-5} \mathrm{~m}$.

Since the working gas $\mathrm{SF}_{6}$ is a non-polar gas, any charge present on the particles will likely remain on them. If they come close enough to each other, these charges will influence their motion. Since the Coulomb force $F_{C}$ decreases with $1 / r^{2}$ with distance $r$ from the charge, dissipation scale dynamics are most likely influenced by $F_{C}$. Lu et al. [5] have introduced the Coulomb turbulence number that relates the Kolmogorov velocity $u_{\eta}$ to the typical velocity $u_{C}=\tau_{P} a_{C}=\tau_{P} F_{C} / m_{P}$ of induced by the presence of charges separated by $\eta$.

$$
\mathrm{Ct}=\tau_{P} \frac{q^{2}}{4 \pi \epsilon_{0} \eta^{2} u_{\eta}}
$$

The measurement of the particle charge within the experiment is difficult. However, the maximum allowable charge given an acceptable value of $\mathrm{Ct}=0.1$ can be calculated. For the particles chosen here (see following section) between $\mathcal{O}\left(10^{3}\right)$ and $\mathcal{O}\left(10^{4}\right)$ elementary charges per particle are acceptable before the charges influence the particle motions. These values are relatively large, but not large enough to exclude charge effects a priori. An important aspect of the data analysis will therefore be the radial distribution function of the particles, since its shape changes dramatically if the particle charge influences their dynamics [5].

Particles of $10 \mu \mathrm{m}$ size are difficult to remove from the tunnel entirely, even though the VDTT features a bypass equipped with high efficiency filter. Therefore, particles must be chosen in such a way that the tunnel remains a safe work place also after the experiment. They cannot be toxic and should ideally be safe to breathe, since particles of size $<10 \mu \mathrm{m}$ can enter the lungs.

\subsubsection{Imaging}

While the first Lagrangian particle tracking setups capable of measuring accelerations in a high- $R_{\lambda}$ flow were silicon-strip detectors from high energy physics experiments [6], modern setups employ high-speed cameras with CMOS (complementary metal-oxide semiconductor) sensors [7]. The charges on their photodiodes are read out in parallel for all pixels, which 


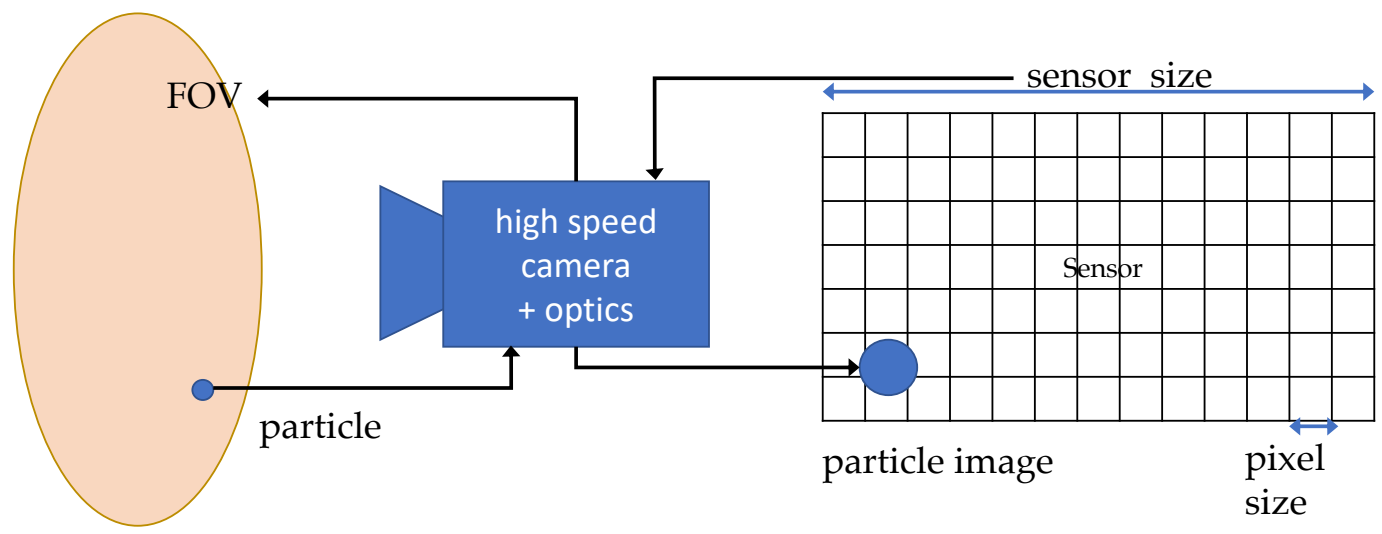

Figure 10.2: Illustration of the limiting conditions for the particle tracking setup. The particle size is approximately fixed at $10 \mu \mathrm{m}$, and the tracking algorithm requires the particle images to be at least $2 \mathrm{px}$ in diameter. This determines the magnification of the camera optics, which together with the sensor size limits the field of view.

allows higher frame rates.

Voth et al. [2] have shown that the reliable tracking of tracer particles requires an oversampling of $\tau_{\eta}$ by more than 1o times. Together with $\tau_{\eta}>0.5$ ms this yields a frame rate of $>20 \mathrm{kHz}$ for the most demanding situations in the experiment.

There exist numerous commercial [8, 9], open source [10], and in-house algorithms capable of tracking particles that move in a turbulent flow [11]. They typically achieve subpixel accuracy, i.e. the particles' locations within a single pixel can be extracted. Nobach et al. [12] have shown that such schemes require particle images $>2 \mathrm{px}$. In this case a precision of up to $0.1 \mathrm{px}$ is possible.

High speed cameras and their optics have a significant aerodynamic influence when placed inside the flow, which has to be avoided. Their closest position to the center of the measurement section cross section is therefore below the tunnel flow.

These considerations in combination with the length- and time scales described in the previous section, and the presence of mean flow quickly show that accelerations can only be efficiently measured by the most advanced high speed cameras available. For example, the Phantom v2511 can record 25000 frames per second at its full resolution of $1280 \times 720 \mathrm{px}$.

The pixel size of these cameras is $28 \mu \mathrm{m}$. Thus, the particle image must be about $60 \mu \mathrm{m}$ to allow efficient subpixel tracking. The image of a spherical 
particle in a optical setup of magnification $M$ is given by

$$
d_{I}=\sqrt{(2 a M)^{2}+d_{A}^{2}}
$$

where $d_{A}$ is the size of its Airy disk

$$
d_{A}=2.44(1+M)(\mathrm{NA}) \lambda
$$

induced by the diffraction due to the system's numerical aperture NA. All illumination sources under considerations are green lasers of wavelength $\lambda=$ 532 or $515 \mathrm{~nm}$. For example, an imaging setup with $M=1$, and $\mathrm{NA}=22$ would yield a particle image of $56 \mu \mathrm{m}$.

Assuming a magnification of $M=1$, the size of the measurement volume can be approximated. At full resolution the longer side of the sensor has 128opx, the shorter $720 p x$, each of size $28 \mu \mathrm{m}$. With $M=1$ the focused measurement volume is as large as the sensor: about $3.6 \mathrm{~cm} \times 2.1 \mathrm{~cm}$.

The knowledge of the measurement volume allows to estimate the approximate time the particles will spend within the measurement volume. This depends predominantly on the mean flow speed $U$, which is typically between 2 and $5 \mathrm{~m} / \mathrm{s}$. If the particles move along the diagonal of the measurement volume, they can be expected to cross it within $\sim 10 \mathrm{~ms}$, i.e. $\sim 1-20 \tau_{\eta}$.

Apparently, the size of the measurement volume is ultimately determined by the size and number of camera pixels and the particle diameter. While the turbulence properties prohibit the use of larger particles, cameras with smaller sensors could be used, such as the Phantom VEO ${ }_{4} \mathrm{k}$, whose pixels are only $6.75 \mu \mathrm{m}$ large. However, these cameras can only acquire $1000 \mathrm{fps}$ at full resolution. Moreover, using single pictures of long exposure would reveal the paths of individual particles as streaks. The available light sources (see next section) can be programmed in such a way that time information could be encoded in their intensity, such that the brightness of these streaks would vary in time. For example, the Phase One XF iXM features 11664px along its longer axis, each only $3.76 \mu \mathrm{m}$ large, allowing a measurement volume of $>0.5$ $\mathrm{m}$ length. The time information of such a setup would not be sufficient to measure accelerations, but the concept provides an interesting path towards measurement of dispersion statistics in a stationary setup. 


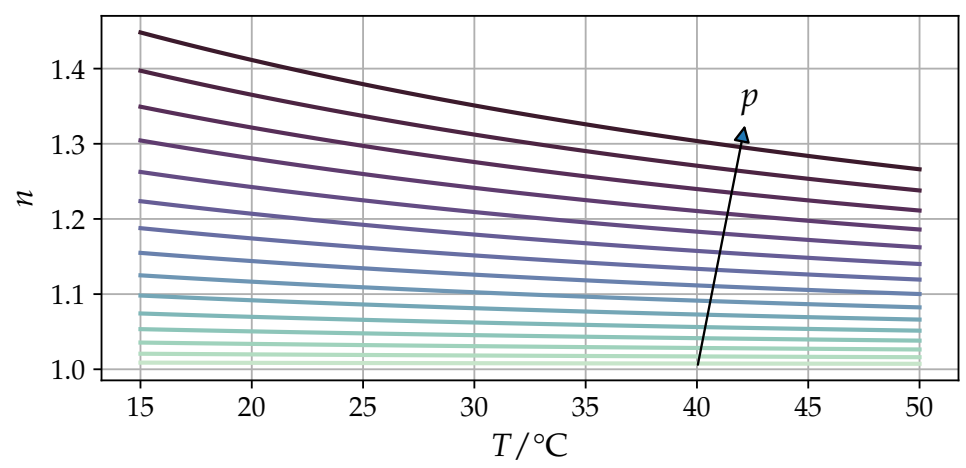

Figure 10.3: Refractive index $n$ for the conditions experienced in the VDTT evaluating the empirical formulas presented in Refs. [13, 14] and using the parameters found by [14]. The refractive index varies severely with pressure and at high pressures also with temperature. Therefore, any optical setup must be adjustable for different pressures and temperature controlled.

\subsection{4 $\mathrm{SF}_{6}$ as a working gas}

The particle tracking setup necessarily has to account for the properties of the working gas $\mathrm{SF}_{6}$ under pressures up to 15 bar. First, any equipment in use has to be vented, i.e. existing pressure differences between the environment and the inside of the device have to be equilibrated. This is the case for most equipment that is not watertight. An exception are the high speed camera sensors, which have a protective glass sealed on, but can be vented by the manufacturer.

The refractive index of $\mathrm{SF}_{6}$ is sensitive to pressure $p$ and temperature $T$. The dependence is fitted in general by [13]

$$
n=1+A \frac{p}{R T}+B(t)\left(\frac{P}{R T}\right)^{2}
$$

using the gas constant $R$. Thomas \& Tayag have determined the fit parameters $A$ and $B(T)$ [14]. For the conditions experienced in the VDTT, the results are illustrated in Fig. 10.3.

The temperature dependence is stronger at larger densities, where even small temperature fluctuations distort an image locally ("barbecue effect"). Further, the refractive index varies by almost 0.5 between 1 and 15 bar. Therefore, any optics focused in air will be strongly out of focus in $\mathrm{SF}_{6}$ at higher pressures. Therefore any refractive optical component inside the tunnel needs to be adjustable. It is advantageous to locate as many of such optical components outside the tunnel to make basic realignment possible and reduce the number of remote controlled devices in the tunnel. 
The VDTT is known to deform under pressure by up to $7 \mathrm{~mm}$ [15]. It is therefore possible, that also light paths (e.g. from an illumination laser) vary between different pressures and should be adjustable.

\subsection{Realisation of a Lagrangian Particle Tracking Setup}

\subsubsection{Particles}

The particles chosen for this setup and fulfilling the requirements defined in Sec. 10.1.2 are cellulose particles commonly used in the cosmetics industry [16]. In particular, KOBO Products Inc.'s CELLULOBEADS USF, D-5, D-10, and D-30 are suited for this experiment. Their properties are listed in Tab. 10.1. The density of the particle material is not known, but its bulk density $\rho_{b}$ is specified. Assuming a porosity $\epsilon \approx 0.4$, the particle density can be estimated

$$
\rho_{p}=\frac{\rho_{b}-\left(\epsilon \rho_{f}\right)}{(1-\epsilon)} .
$$

\begin{tabular}{|l|c|c|}
\hline & Diameter $(\mu \mathrm{m})$ & Approx. Density $\left(\mathrm{kg} / \mathrm{m}^{3}\right)$ \\
\hline USF & 4 & 270 \\
\hline D-5 & 10 & 1000 \\
\hline D-10 & 15 & 1200 \\
\hline D-30 & 30 & 1400 \\
\hline
\end{tabular}

Table 10.1: Properties of particles considered in this study. Diameters taken from their datasheet [16], density estimated from the bulk density assuming a packing fraction of 0.4

With this information, the expected Stokes number eq. (10.2) for different values of the turbulent dissipation rate $\varepsilon$ can be estimated. Fig. 10.4 shows the Stokes number estimated from the particle data and the fluid properties [17]. The errorbars indicate the uncertainty in the Stokes number due to an error in the packing fraction by \pm 0.2 . The only particles that yield $\mathrm{St}<0.1$ for all situations considered, are USF particles. However, due to their small size, they require very strong illumination to be visible. D-30 particles are in the inertial regime (St>0.1) in all situations. D-5, and D-10 particles provide a compromise between inertial effects and illumination requirements. In general, lower pressures, low turbulence intensities, and large integral scales $\left(\varepsilon \sim u^{3} / L\right)$ are most likely to create situations, where the inertial effects on the particle motion are negligible. Incidentially, the wider variety of available 

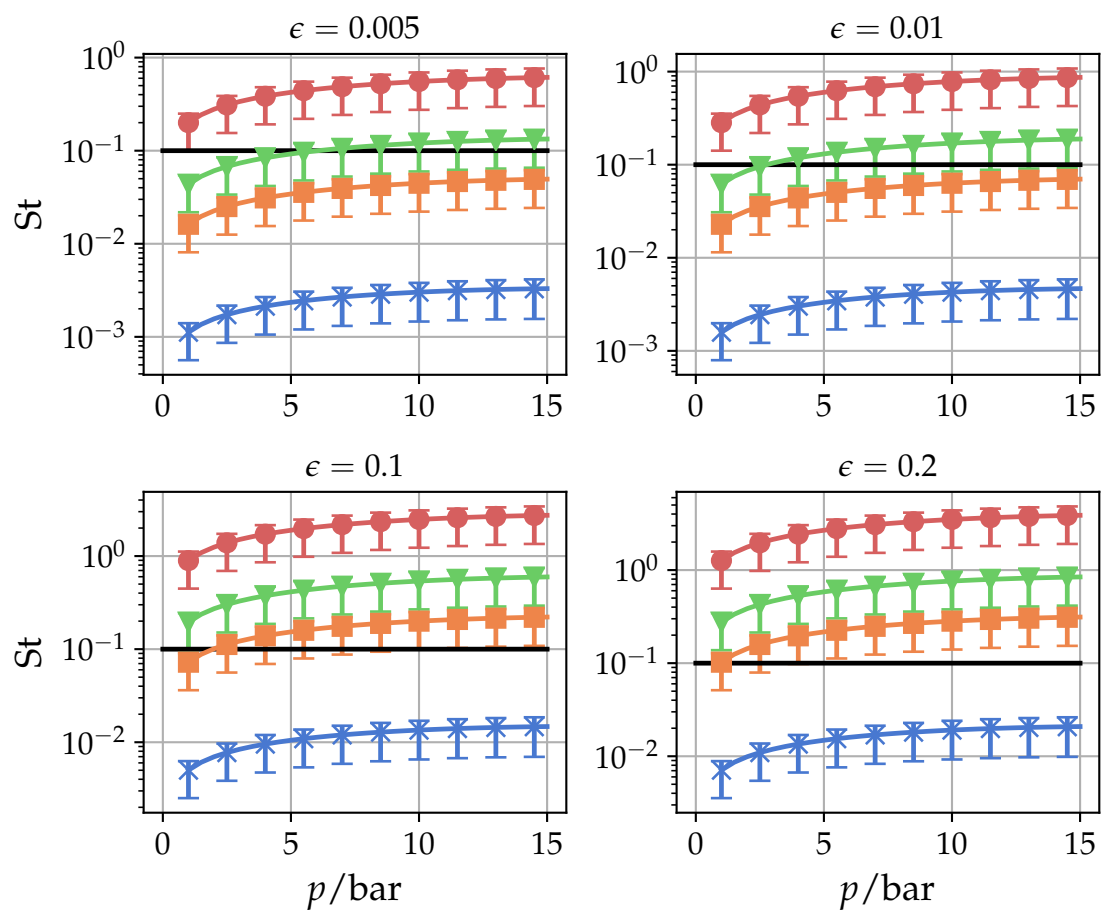

Figure 10.4: Stokes number defined in eq. (10.2) as a function of the facility pressure for different values of the turbulent dissipation rate $\sim u^{3} / L$. Red circles: D-30; green triangles: D-10; orange squares: D-5; blue crosses: USF. The critical value of $\mathrm{St}=0.1$ is indicated by a black line. Error bars indicate uncertainty due to unknown particle density.

Stokes numbers is exciting in its own right and allows studies on the inertial particles in turbulence. This has a great relevance to atmospheric processes, where water droplets with St>1 play a crucial role.

The most important danger in handling any of these particles is their inflammability and the associated risk of a large dust explosion. Therefore, the particles are stored in a fire safe board and significant quantities are only dispersed in the abscence of oxygen (i.e. in the $\mathrm{SF}_{6}$-filled wind tunnel). Otherwise, these particles provide no particular known health hazard.

The dispersion of particles is an essential part of any particle tracking setup. The mechanism has to be designed, such that a controllable amount of monodisperse particles is discharged homogeneously into the measurement volume. Sampling the entire wind tunnel in such a way would be ideal, but requires large amounts of particles, which need to be replenished frequently. From initial experiments, the author estimates that $>50 \%$ of particles get are removed from the flow per tunnel revolution. For this reason, the particles are dispersed about $5.0 \mathrm{~m}$ upstream of the measurement volume, and 0.485 $\mathrm{m}$ above the floor of the measurement section. The dispersion mechanism is illustrated in Fig. 10.5. A strong pipe flow into the wind tunnel is created, 


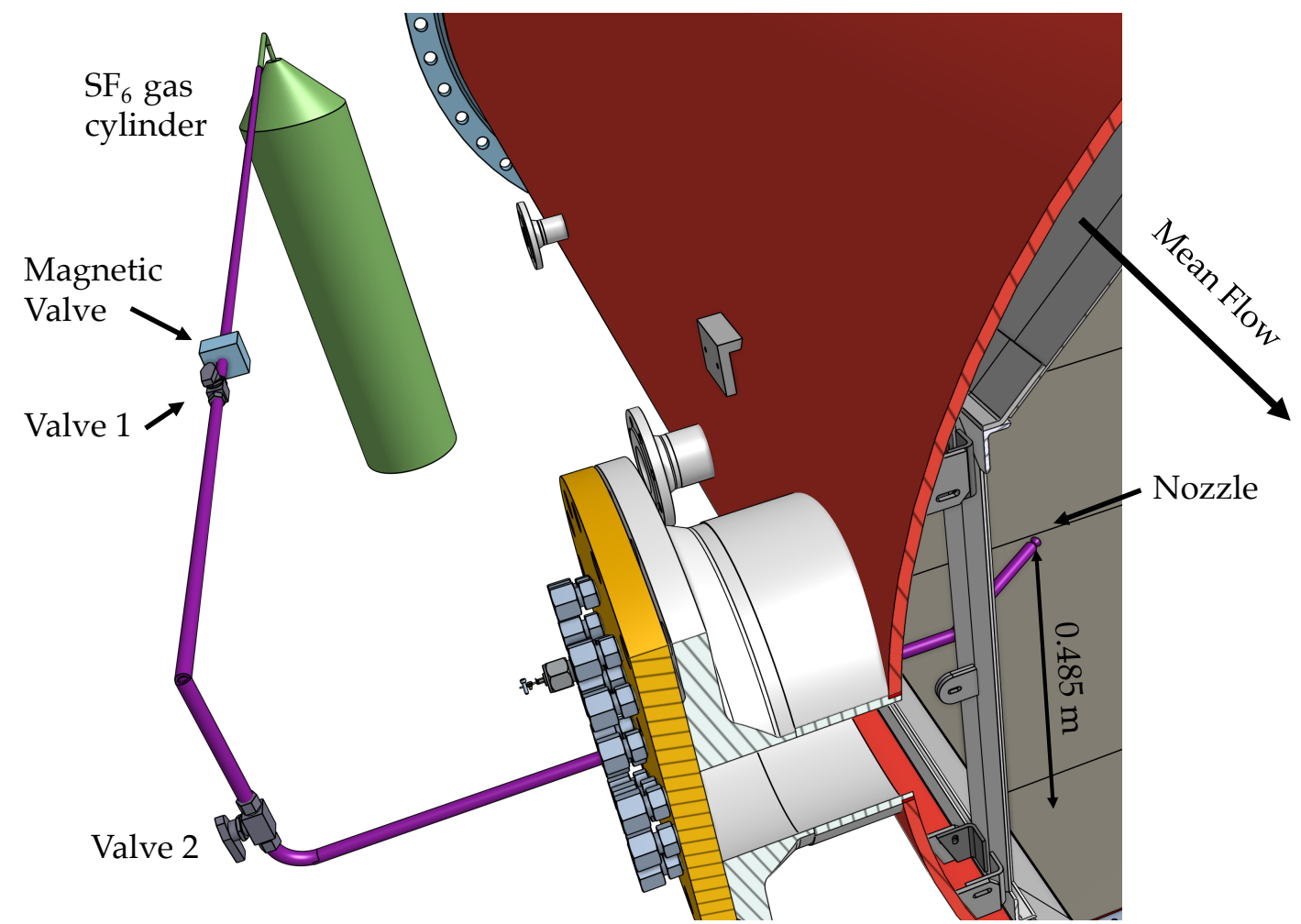

Figure 10.5: Illustration of the particle dispersion system. The particles are initially filled directly into the pipes that connect valves 1 and 2 . The gas bottle delivers $\mathrm{SF}_{6}$ at pressures $\approx 5$ bar higher than the wind tunnel pressure. When valves 1,2 , and the magnetic vale are open, a jet emerges at the nozzle that carries the particles within the pipes into the tunnel. The nozzle points about $45^{\circ}$ upstream.

which is ultimately discharged into the flow through a nozzle and carries with it particles that are within the pipe. The source of the pipe flow is a $\mathrm{SF}_{6}$ gas cylinder, whose effective pressure is controlled by an attached pressure regulator to about $\Delta p_{\text {Injection }} \approx 5$ bar above the current wind tunnel pressure. A section of the pipes that connect gas cylinder and wind tunnel flow can be isolated by two manual valves. It can then be opened safely and particles can be filled directly into the pipes. Once resealed, the two manual valves can be opened. The discharge of particles is initiated by an additional magnetic valve (1) (numbers in circles indicate parts found in Appendix A). It is operated by a Berkeley Nucleonics Corporation 555 pulse delay generator (2). Its TTL (transistor time logic) signal initiates two relais, which open the magnetic valve.

The number of particles discharged by this techniques depends on the number of particles within the pipes (decreases with time after replenishment), the duration for which the magnetic valve allows flow, and the pressure difference between the gas cylinder and the wind tunnel. Below pressure 

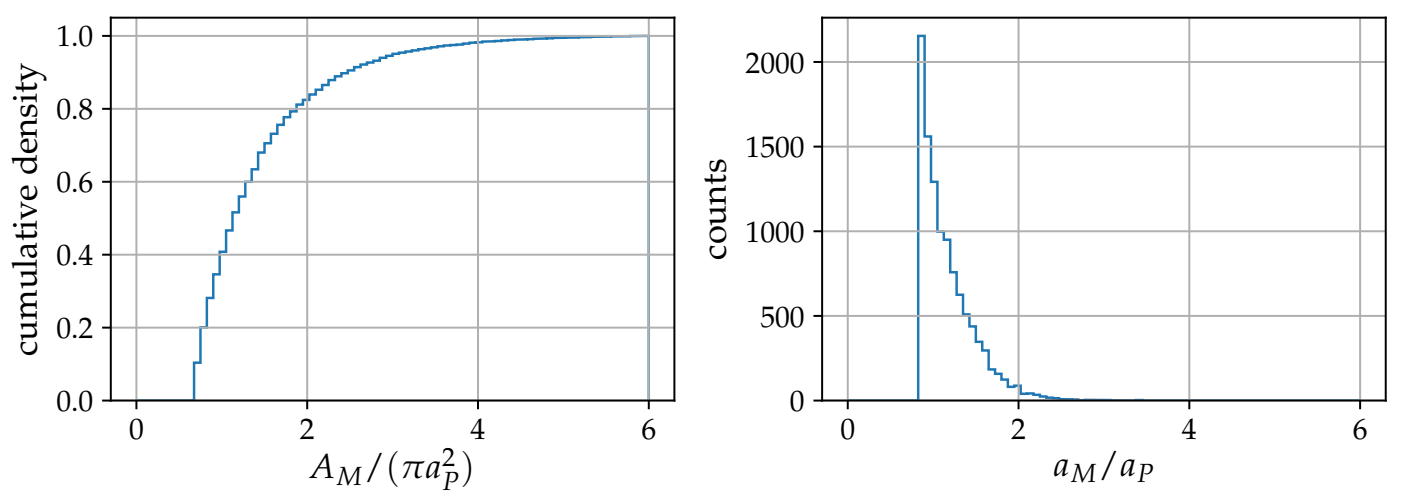

Figure 10.6: Measurement of the particle size distribution. Left: cumulative probability density showing that $80 \%$ of detected particles are smaller than two particle diameters. Right: number of particles at a given diameter $a_{m}$ normalised by the single particle theoretical diameter $a_{p}$.

differences of 4 bar, the majority of the particles remains so close to the measurement section wall that they get trapped within its boundary layers. If the flow leaving the nozzle is too strong, the actual wind tunnel flow might be strongly affected. A rough guidance from experience could read: $30 \mathrm{ml}$ of particles last for about 60 injections at jet durations between 0.3 and $0.7 \mathrm{~s}$.

An important property of any particle dispersion mechanism is the size distribution of the resulting particle cloud, i.e. how many clumps of two or more particles are ejected. Since realistic experiments involving the setup are only possible inside the $\mathrm{SF}_{6}$-filled wind tunnel, and a large magnification is necessary to distinguish single particles from doublets, the following experiment was carried out to estimate the particle number of multi-particle clusters ejected: After particle tracking experiments using D-1o particles were carried out ( $p=14.9 \mathrm{bar}, \Delta p_{\text {Injection }}>5 \mathrm{bar}$ ), the tunnel was evacuated and filled with air. In this case, an apparently single layer of particles is clearly visible on the tunnel floor. A black tape was carefully stuck onto the particlecovered tunnel floor and removed again. On the area where the tape had been located, all particles were picked up by the tape. The tape was then placed under a Keyence VK-X210 confocal $3 \mathrm{D}$ laser scanning microscope and an area of $\approx 11.5 \mathrm{~mm} \times 9.1 \mathrm{~mm}$ was imaged. The image was processed in ImageJ (Despeckle, Threshold, Median Filter $(r=0.8 \mathrm{px})$ ) and the particles were measured using the built-in algorithm. Detected particles with a size $<120$ $\mu \mathrm{m}^{2}$ and a circularity $<0.30$ were rejected. A part of the original image, the reduced version with detected particles indicated, and the particle size statistics are shown Fig. 10.6.

The measured projected area of the particle $A_{M}$ was predominantly smaller 
than $2 \times$ the expected area of the particle given its nominal radius $a_{P}$. Since no peaks at integer multiples of the nominal particle size are visible, a significant amount of noise and erroneously detected particles is expected. Even after denoising, the picture was heavily influenced by the glue structures on the tape, which could not be removed entirely by thresholding and size filtering. Nevertheless, most objects detected by the algorithm were particles. The right panel shows that only a very small number of objects has radii that exceed that of the particles. It is therefore concluded that the appearance of clumps with significantly enhanced Stokes number is relatively unlikely and will only marginally influence measurements, at least when using D-10 particles.

In summary, a simple particle dispersion system was built that allows for automated release of particles into the wind tunnel, particle replenishing while the VDTT is filled with $\mathrm{SF}_{6}$, and produces predominantly monodisperse particle clouds. A more sophisticated system using liquid $\mathrm{SF}_{6}$ in a separate pressure vessel stirred constantly by a cooling fan proved to be too complicated for efficient operation.

\subsubsection{Imaging}

As indicated in Sec. 10.1.3, the imaging system should be placed as close to the measurement volume as possible (usually close to the center of the tunnel cross section), but outside the mean flow. Given the constraints of camera size, resolution, and the associated need for large focal lengths, the tunnel offers only two possible locations for high-speed cameras: Below the measurement section floor utilising mirrors, and the manholes. However, the manholes can only carry compact camera arrangements, i.e. are unsuited for the ultra high speed cameras required to measure Lagrangian accelerations.

A sled previously built to allow particle tracking in a comoving reference frame was adjusted to accommodate four Phantom v2511 high speed cameras (3)-6. The platform consists of an aluminium plate, which is freely movable for about $2.5 \mathrm{~m}$ in the streamwise direction along two rails mounted inside the wind tunnel. The cameras and their optics are placed on two steel plates, which are connected firmly by two arms to avoid relative motion of the cameras. This arrangement of steel plates is connected to the aluminium base plate through springs (see Fig. 10.7). This way, the cameras are mechanically decoupled from the tunnel, which vibrates considerably during operation. Such vibrations would severely impact the measurements, since already a vibrational displacement of $10 \mu \mathrm{m}$ corresponds to a noise level of $1 \eta$. 


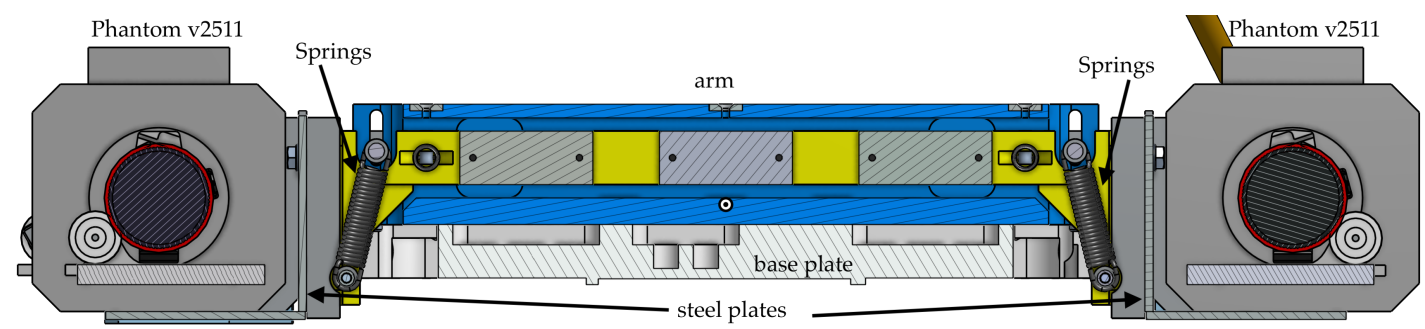

Figure 10.7: Cut through the camera platform. The four cameras (two visible here) are mounted on L-shaped steel camera supports, which are connected by two arms. This arrangement is connected through springs to the main platform, which is connected to the wind tunnel rail system. This way, the cameras are mechanically decoupled from vibrations during operation of the wind tunnel.

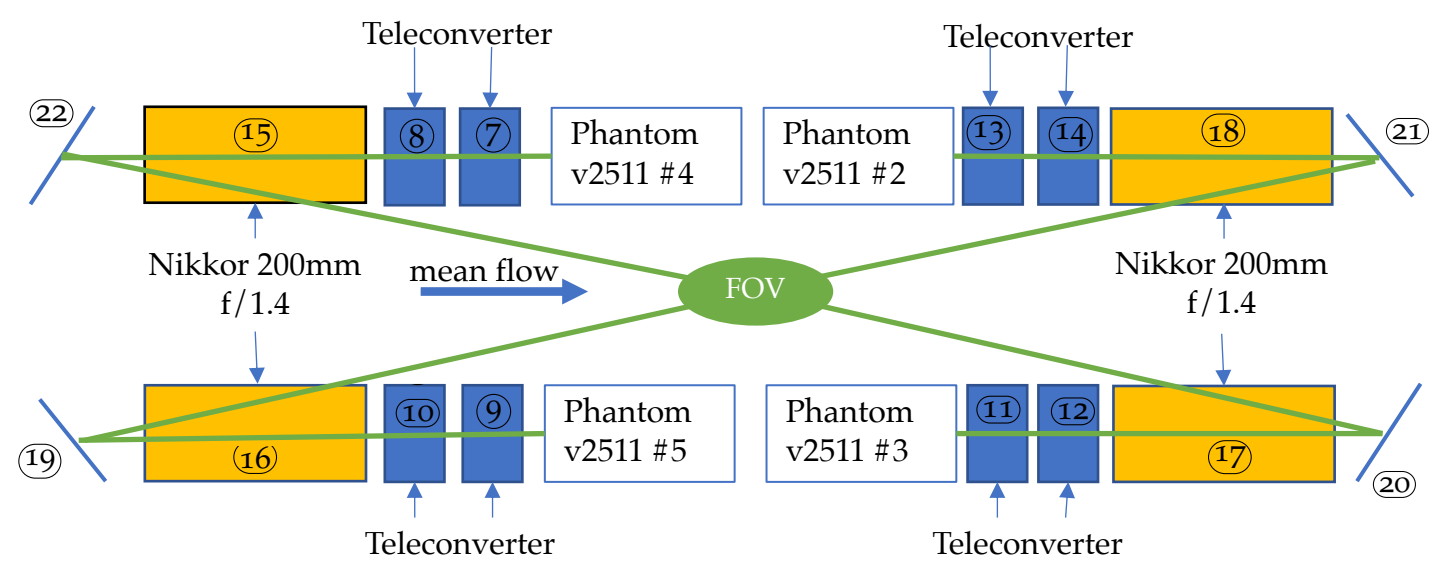

Figure 10.8: Schematic (top view) of the optics arrangement for the imaging. A magnification $M \approx 1$ is achieved by combining a $200 \mathrm{~mm}$ lens (15)-(18) with two $2 \times$ teleconverters (7)-(14) . Adjustable mirrors (19)-22) on each camera guide the light from the field of view (FOV) into the camera optics.

The considerations presented in Sec. 10.1.3 lead to the requirement that the optical magnification $M=1$ to obtain particle images usable for Lagrangian tracking. The optical setup to achieve this magnification is illustrated in Fig. 10.8. Each camera has two teleconverters with $2 \times$ magnification and a $200 \mathrm{~mm}$ Nikkor lens mounted to it. The cameras are pointing towards mirrors, such that they can observe the measurement volume. The mirrors are mounted in kinematic mirror mounts (25), (26) that can be remote controlled through piezo motors.

As outlined in Sec. 10.1.4, the refractive index of $\mathrm{SF}_{6}$ depends sensitively on the pressure. Therefore, the effective focal length of the camera optics changes and needs to be compensated remotely. Since the chosen combination of lenses and cameras only supports manual focusing on the lens itself, the following mechanism was implemented: A timing belt is tightly fixed around each lens with its teeth pointing outwards. A gear 23 is attached to the timing belt and operated by four stepper motors (24), which are controlled by 


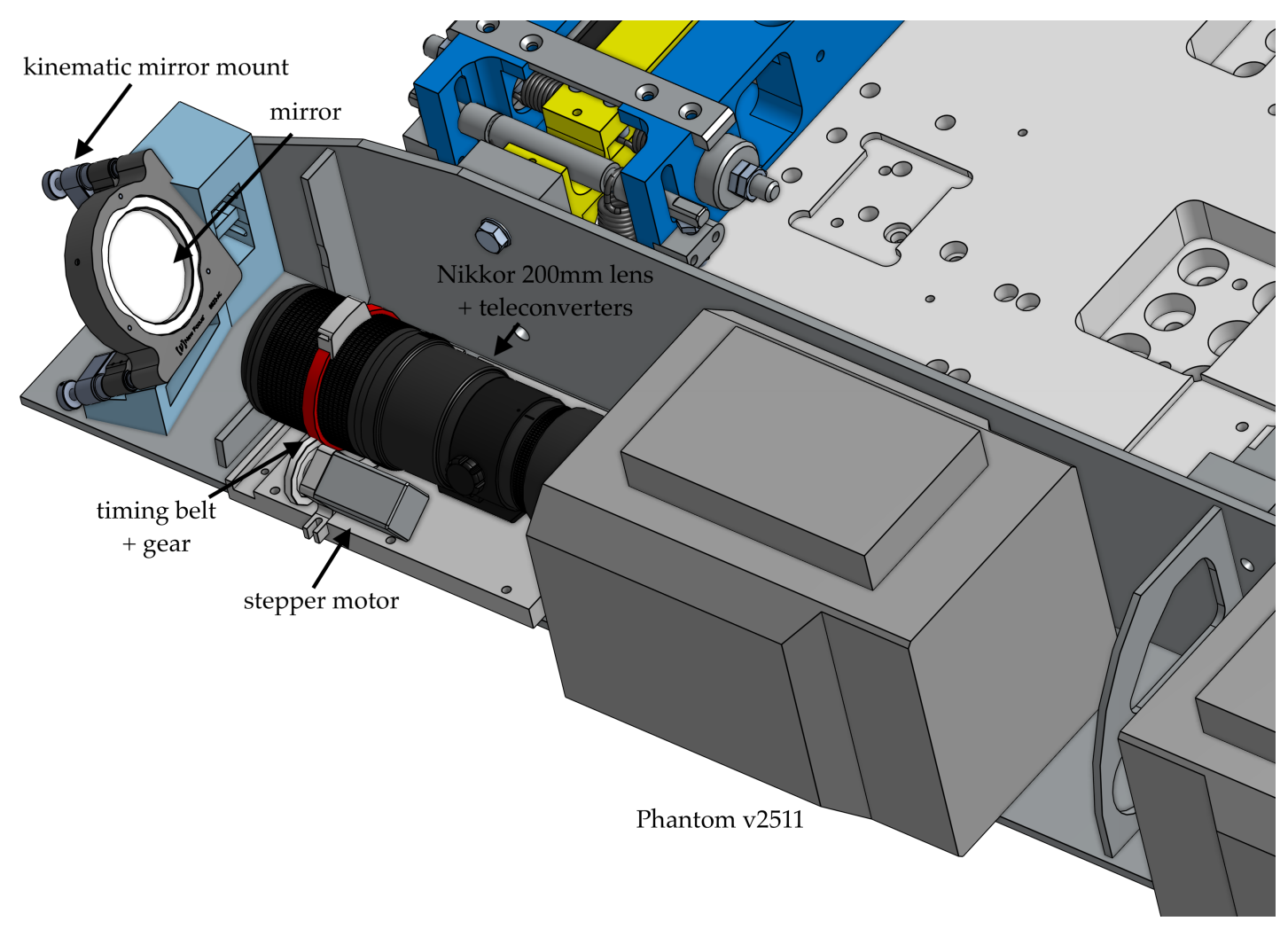

Figure 10.9: Illustration of a single camera optical setup. The timing belt and gear 23 transmit the stepper motor (24) rotation to the focus of the lenses. The kinematic mirror mount provides the view to the measurement volume, which lies behind the camera.

an Arduino Uno.

The positions of the particles recorded in units of pixels on the two- dimensional sensor need to be converted into units of meters in the threedimensional measurement volume. To achieve this, several camera calibration models have been invented in the past. For high-precision measurements, only systematic calibrations using a pre-defined mask in a separate procedure can be used. In general, a suitable camera calibration consists of two matrices: the intrinsic matrix, and the extrinsic matrix. The intrinsic matrix contains information about the camera optics and therefore depends on the index of refraction. The calibration of the camera intrinsics must be performed in-situ at every ambient pressure. To this end, the existing hot wire traverse (see Sec. 4.I) is equipped with a calibration mask. This traverse can be moved in the streamwise direction and has an additional vertical traverse mounted. The calibration mask mounted on the vertical traverse can therefore be moved into the measurement volume for calibration, the position of the mask relative to the cameras can be changed, and the traverse can be moved downstream of the measurement volume after calibration as to not influence the flow during measurement. Because the movement of the calibration mask is limited to two 
directions, images where the calibration plate is at the corners of the image can only be captured when parts of the pattern are obstructed. In this case, the calibration is only possible when the visible area of the calibration pattern can be identified. This is possible for so-called ChAruco patterns, where the white areas of a checkerboard pattern are equipped with a pixel code (ArUco pattern [18]). These patterns are taken from a standard library and can be read out by existing calibration algorithms, implemented, e.g. in the OpenCV library [19] to obtain a direct correspondence between a detected checkerboard corner on the image and the real-world corner on the calibration plate. This way, also calibration images with only partially visible patterns can be used [20]. The calibration pattern for the intrinsic calibration is a chemical development of a digitally created ChAruco pattern, which was mounted on a flat aluminium plate by the manufacturer. The pattern has a size of $8 \times 8$ fields of marker size $3 \mathrm{~mm}$ taken from the original ArUco library [18]. It is depicted in Fig. 10.10.

The extrinsic matrix consists of a rotation matrix and a translation vector, which define the position of the camera with respect to an arbitrarily chosen world coordinate system. Three possible strategies exist within the current setup to obtain the extrinsic matrix. The calibration matrix can be placed at well-known positions with respect to the chosen coordinate system origin. The traverse system described above does not offer the accuracy required to perform such a calibration in-situ. In principle calibration schemes that work without knowledge of the calibration mask position [21, 22] can be used to obtain the camera intrinsic matrix. The most widely employed of such schemes is Zhang's method. It requires at least three views of a flat, asymmetric calibration plate with patterns of known dimensions, but to achieve the precisions required here, more views are needed. Most importantly, the position or orientation of the calibration plate is not needed.

The camera extrinsic matrix is independent of the refractive index (it only contains geometric information about the camera locations) and can therefore be obtained in air. When in air the calibration mask can be positioned freely in space and Zhang's method can be used. In addition, a temporary setup can be employed, which performs a one-time extrinsic calibration where the position of the calibration mask in space is known. For this, a calibration target is mounted on a manual high-precision horizontal stage.

The calibration plate is mounted on a aluminium holder, which can be moved remotely in streamwise and vertical directions. The holder is also equipped with two LED strips. Along with an additional LED strip located 

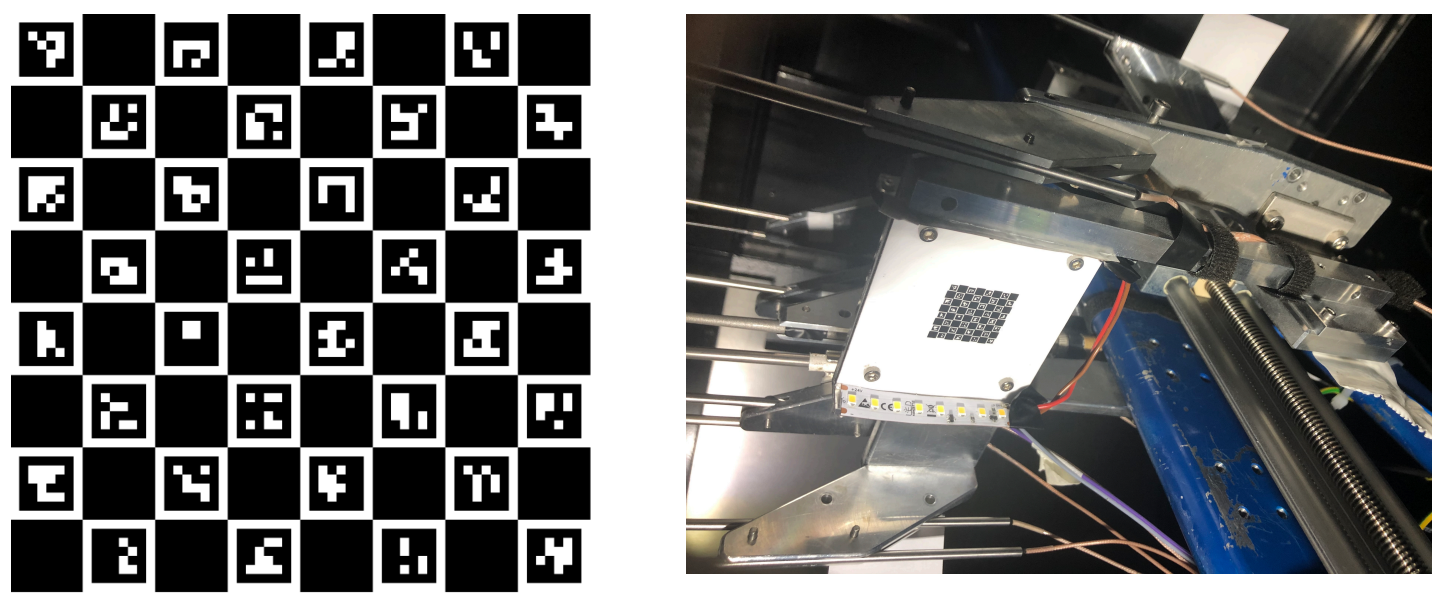

Figure 10.10: Left: Calibration pattern used to calibrate the cameras. The use of a ChArUco pattern allows the inclusion of only partially visible calibration images. Right: Looking up at the calibration plate mounted on its holder with LED-Strips for illumination. The holder is mounted on a vertical traverse

\begin{tabular}{|c|c|c|c|c|}
\hline$d_{1}$ & $f_{1}$ & $d_{2}$ & $f_{2}$ & beam size in measurement volume \\
\hline $63 \mathrm{~mm}$ & $62.9 \mathrm{~mm}$ & - & - & $90 \mathrm{~mm}$ \\
$30 \mathrm{~mm}$ & $75 \mathrm{~mm}$ & 50 & $200 \mathrm{~mm}$ & $70 \mathrm{~mm}$ \\
$80 \mathrm{~mm}$ & $75 \mathrm{~mm}$ & - & - & $60 \mathrm{~mm}$ \\
$100 \mathrm{~mm}$ & $100 \mathrm{~mm}$ & - & - & $50 \mathrm{~mm}$ \\
\hline
\end{tabular}

Table 10.2: Possible beam forming optics. Focal lengths and distances $( \pm 5 \mathrm{~mm})$ on the optical axis from Fig. 10.11

on the measurement section floor directly underneath the particle tracking volume, this provides the illumination during calibration when the laser illumination would pose a risk to the hot wires installed close to the calibration pattern.

\subsubsection{Illumination}

The light emitted from an illuminated source scales with its surface area, i.e. with the square of its length scales. Therefore, particles for Lagrangian tracking in an environment where the Kolmogorov scale is particularly small, need a strong illumination. The setup presented here utilises a Trumpf TruMicro $7642 \mathrm{Nd}$ :YLF laser 28 with a maximum output power of $300 \mathrm{~W}$ at $100 \mathrm{kHz}$ pulse frequency and an energy per pulse of up to $7.5 \mathrm{~mJ}$. The laser light is coupled into an optical fiber (29), which guides the light towards the optical setup. The optical setup is based on a optical breadboard enclosed by aluminium plates. It is mounted on two rails connected to the tunnel to avoid large relative motions between the wind tunnel during operation and the optical setup. The laser fiber head can be mounted to the cavity such that no 


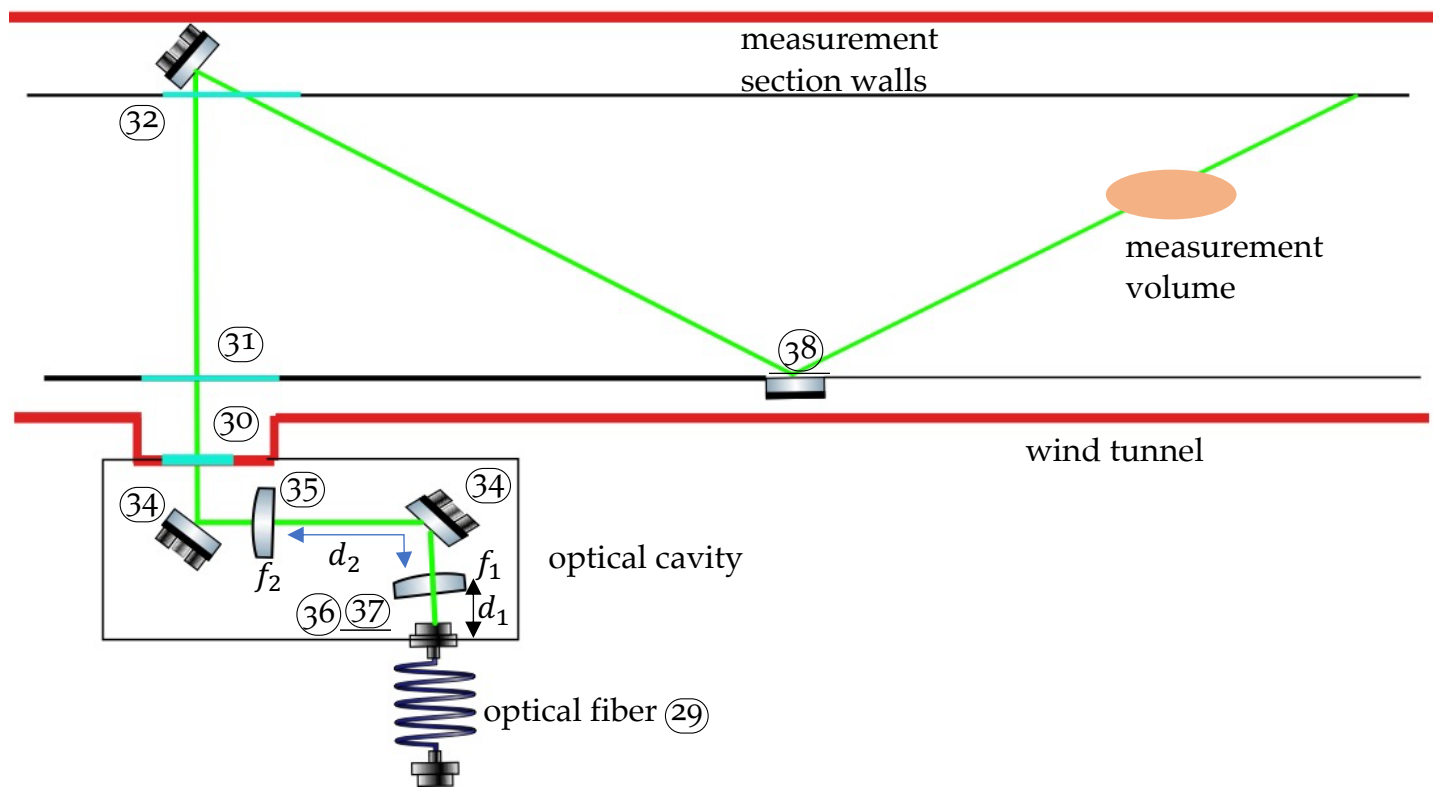

Figure 10.11: Illustration of the beam path starting from the optical fiber (29), through two lenses of focal lengths $f_{1}$ and $f_{2}$. The mirrors (34) in the optical cavity can be adjusted to guide the beam path through windows (31) and (32) onto another mirror mounted on a remote controllable mirror mount (25). The beam is then guided to a large mirror (38), and through the measurement volume to a metal plate

light can leak except for the specified openings.

The laser light enters the cavity at an opening angle of $5.91 \pm 0.2^{\circ}$ (A. Bertens, personal communication, Jun 26,2019 ) and needs to be collimated. Three optical setups of collimation were investigated and the results are listed in Tab. 10.2. The first lens in the optical setup needs to be slightly tilted to avoid back reflections to hit the optical fiber or couple back into the laser cavity. The beam is further guided in such a way that the reflection from the pressure sealed window (30) is not focused back into the fiber. The outlet of the optical cavity is mounted as close to the pressure sealed window (30) as possible and the remaining gap is covered by laser safety fabric. Therefore, all active optical elements of the beam forming setup are outside the pressure vessel and thus unaffected by the change in index of refraction. The beam enters the measurement section through another high-efficiency window (31). Once inside the tunnel the beam can only be moved by a single remotely controllable kinematic mirror mount (25). This mirror is aligned in such a way that the beam hits another, very large mirror, from which it enters the measurement volume. The beam is terminated at a black steel plate. The area where the beam hits this steel plate is marked before pressurising the wind tunnel, and observed by a USB camera. 


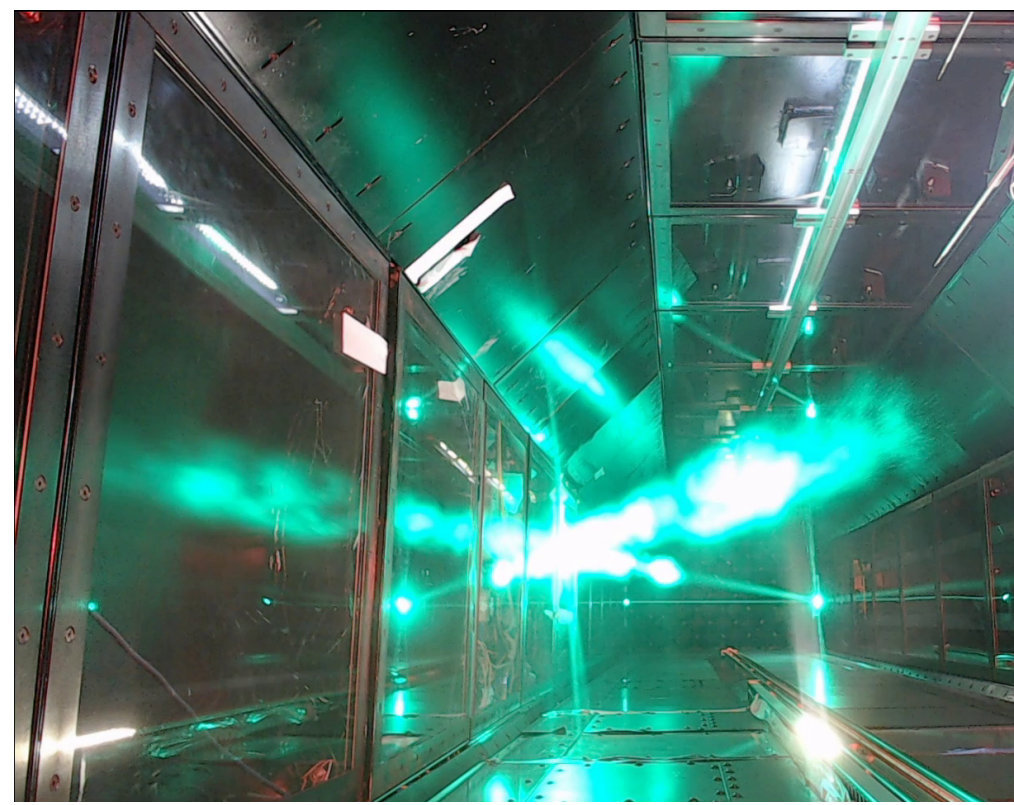

Figure 10.12: Image from a conventional camera taken during a typical experiment looking upstream. The overexposed parts of the green laser beam are regions of high particle density. Note that the laser beam was wider during this experiment than described here. The measurement volume is above the white light visible in the front of the picture.

\subsubsection{Operating Procedure and Data Transfer}

An experiment is typically initiated by a BNC Model 555 pulse delay generator (2). It first opens the magnetic valve (1) and initiates a particle discharge into the tunnel. The second output is connected to the $24 \mathrm{~V}$ digital inputs of the laser 28. It is configured in such a way that laser pulses of a certain energy are released for a pre-defined duration. The pulse frequency of the laser can be provided to the FSYNC port of the high speed cameras, such that frame rate and laser frequency are synchronised. Double exposures are possible through a frequency divider. The third output of the pulse delay generator is connected to the trigger input of the high speed cameras. This way, the release of particles, the start of the illumination, and the trigger of a recording can occur in quick, and adjustable progression.

Each recording of all four cameras yields $>80$ GB of data. The data can be reviewed and downloaded directly to a server with a storage capacity of $35 \mathrm{~TB}$ over 1oGbit ethernet. This computer serves as an intermediate storage. Once a measurement session is finished, the data can be transferred to the institute storage system.

Four USB webcams observe the experiment. In particular, the laser beam path can be controlled through these cameras. Beam displacements can be detected and adjusted using a kinematic mirror. 
The situation in the measurement section during a typical experiment is illustrated in Fig. 10.12. The laser beam enters as a relatively narrow beam, hits the first mirror 33 behind the right row of windows, then the second mirror 38 on the left row of windows. The clouds of particles are visible as overexposed portions of the beam. The measurement section is located above the LED strip in the front of the picture. 


\section{Chapter 11}

\section{Results of Particle Tracking Experiments in the VDTT}

This chapter presents the results of initial particle tracking experiments in the VDTT. The study was designed to demonstrate the capability of the particle tracking system in the pressurised facility, in particular its potential to obtain statistics of velocity and acceleration of particles in the extremely turbulent flow.

\subsection{Characterisation of the Data and Data Analy- sis}

Three recording sessions consisting of approximately 10 videos each of particle tracking data were performed with the setup described in Ch. 10. The facility pressure was $4.92 \mathrm{bar}$ in all cases $\left(31.3 \mathrm{~kg} / \mathrm{m}^{3}\right)$ and the Reynolds number was altered by changing the active grid actuation. Tab. 11.1 provides an overview over the measurement conditions. The number of particles visible in one frame varied strongly throughout the video as shown in Fig. 11.2. We attribute this to the particle injection mechanism, which was close to the wall to minimise the flow disturbance by the nozzle. We suspect that the many particles got trapped in the sidewall boundary layer and consequently did not pass the measurement volume. Furthermore the injection mechanism was found to eject particles intermittently. Therefore, the timing between the particle injection and camera trigger must be relatively finely tuned to capture as many particles as possible.

The varying particle density in the measurement volume illustrated in Fig. 11.2 causes a severe sampling bias towards those parts of the flow that happen 


\begin{tabular}{|l|c|c|l|l|l|l|c|}
\hline$R_{\lambda}$ & $P / \mathrm{bar}$ & $U /(\mathrm{m} / \mathrm{s})$ & Grid & $N_{\text {tracks }}$ & fps & $\tau_{\eta} / \mathrm{ms}$ & $\varepsilon /\left(\mathrm{m}^{2} / \mathrm{s}^{3}\right)$ \\
\hline 2180 & 4.92 & 6.07 & $\begin{array}{l}\mathrm{LT}_{3} \mathrm{LT} 2 \\
\mathrm{RMS}_{50}\end{array}$ & $\begin{array}{l}39875 \\
(12227)\end{array}$ & 25000 & 0.76 & 0.81 \\
\hline 2740 & 4.92 & 5.99 & $\begin{array}{l}\text { LT5LT3 } \\
\text { RMS50 }\end{array}$ & $\begin{array}{l}61659 \\
(19135)\end{array}$ & 25000 & 0.93 & 0.61 \\
\hline 3460 & 4.92 & 5.76 & $\begin{array}{l}\text { LT6LT1 } \\
\text { RMS50 }\end{array}$ & $\begin{array}{l}110860 \\
(27303)\end{array}$ & 25000 & 1.33 & 0.50 \\
\hline
\end{tabular}

Table 11.1: Overview of particle tracking experiments. Numbers in brackets denote the number of tracks whose length exceeded the filter length and thus allowed the calculation of velocity and acceleration. FPS is the number of camera frames captured per second. For details on the grid forcing algorithms see Sec. 4.2.

to be particle-laden.

The videos were analysed with the in-house particle tracking code by Dr. Jan Molacek. It provided tracks consisting of time, three-dimensional position, and tracking errors. Due to the relatively low seeding density and high distortion of the optical transfer function, the subpixel positions of the particles on the camera sensors were found using a simple blob detection. The three-dimensional triangulation is inspired by the Shake-the-box (STB) algorithm [9]. This involves a polynomial extrapolation of existing tracks to obtain a new candidate position. These positions are then "shaken" around in space until the difference between the images observed on the camera sensors and those projected by a model is minimised. Our code optimises all image properties on a given sensor simultaneously instead of individually like the STB algorithm. The final particle positions in $3 \mathrm{D}$ space are then obtained by standard line-of-sight triangulation. Furthermore, the code generalises the extrapolation step by considering multiple possible extensions of each trajectory at once. This reduces the requirements on the temporal resolution and makes tracking of particles with larger position uncertainty or higher acceleration possible. The algorithm further requires stereo-matches to be unique and thus removes "ghost" particles. The number of particles found this way is given in Tab. 11.1 and example tracks are shown in Fig. 11.3. Positions with a triangulation error (sum of squared residuals between measured sensor position and reprojected sensor positions) of $>1.5 \mathrm{px}^{2}$ were rejected. Gaps within the tracks (through the aforementioned rejection or failed triangulation) smaller than 16 frames were interpolated through a cubic spline to obtain continuously sampled tracks. The longest tracks sampled in this way were about $7 \tau_{\eta}$ long. It is therefore clear that detailed investigations of the Lagrangian inertial range require lower mean flow velocities to increase the residence time of 

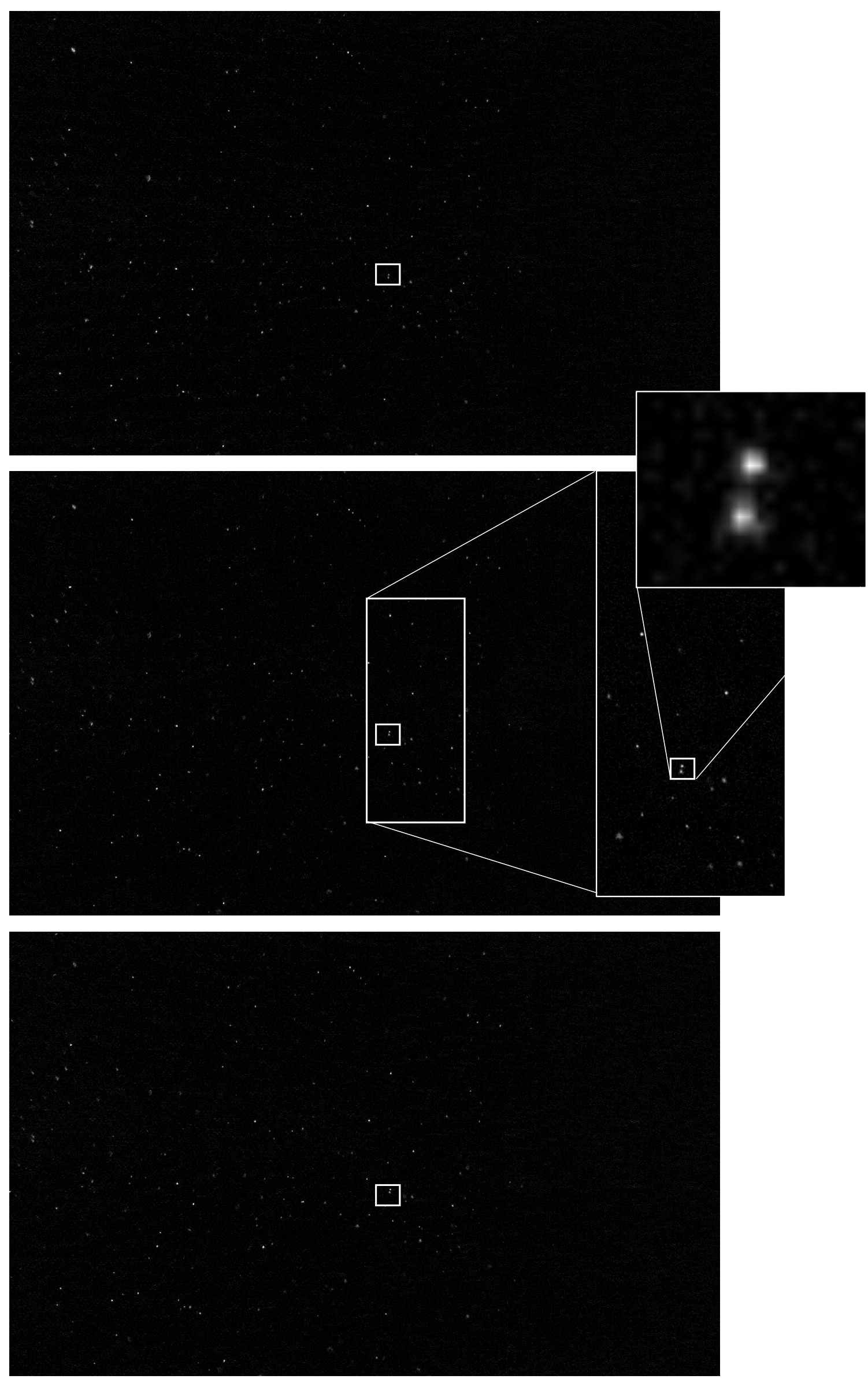

Figure 11.1: Typical sequence of three consecutive frames. 

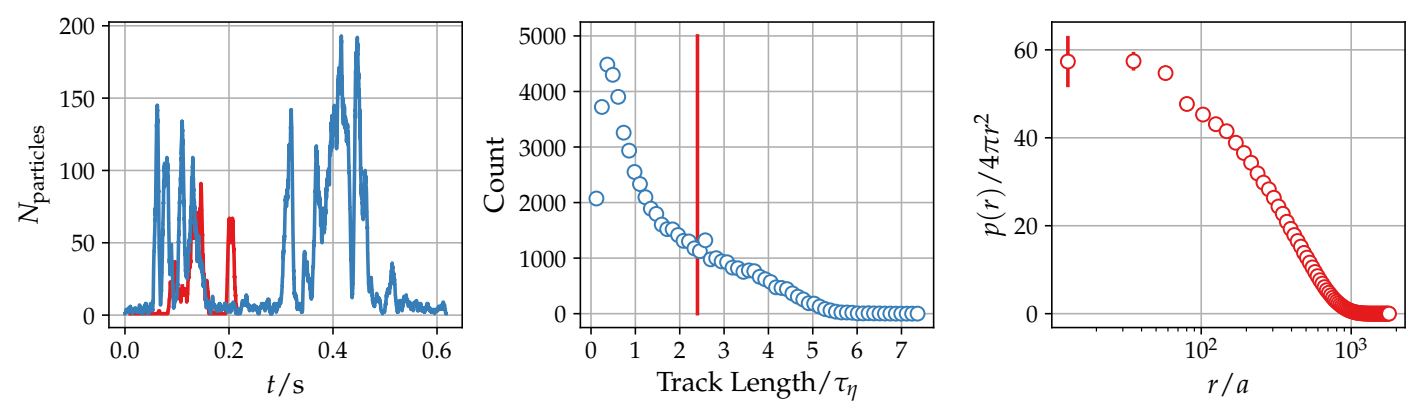

Figure 11.2: Left: Two examples of particle numbers as a function of acquisition time from Experiment 3. The flow is very sparsely seeded for the majority of the acquisition time with only relatively short periods with >10 particles in the field of view. Middle: Distribution of track lengths. The red line denotes the length of the chosen differentiation filter. Tracks right of it are suitable for acceleration and velocity measurements. Right: Normalised probability of finding a particle a distance $r$ away from another particle. The abscissa was normalised by the particle diameter $a$.

the particles in the measurement volume.

To obtain statistics of acceleration and velocity in the Lagrangian sense, the tracks were differentiated by convolving them with a (two times) differentiated Gaussian. This yields a smoothed estimate of the velocity and acceleration. The convolution kernel was normalised according to Mordant et al. [23] to account for the finite filter length $L_{f}$ at a given filter width $w_{f}$. This method was recently compared to the application of penalised splines [24]. It was found that the Gaussian differentiation introduces a significant bias in comparison to such splines and reference data from numerical simulations. The Gaussian differentiation has been chosen here nevertheless to obtain a preliminary set of data. A relatively small filter width of $w_{f} \approx 0.2 \tau_{\eta}$ (7 frames, filter length 60 frames) was chosen to minimise the filter bias. As expected, increasing $w_{f}$ reduces the acceleration variance $\left\langle a^{2}\right\rangle$ (not shown here).

The typical quantities characterising a turbulent flow such as $R_{\lambda}$ or $\eta$ rely on the value of the turbulence dissipation rate $\varepsilon$. While its extraction from velocity time series or fully sampled velocity fields is a standard procedure (cf. Sec. 5.3.3), its estimation from sparse Lagrangian particle tracks is much more difficult [VOTH2002]. For this demonstration of the tracking system, we use the relations between the experimental parameters (fluid density, mean flow speed, and active grid) and the resulting turbulence parameters shown in Sec. 4.2 and Ch. 9. From the active grid forcing we estimate $L=V_{\text {corr }}^{1 / 3}=$ $\left(\sigma_{S}^{2} \sigma_{t}\right)^{1 / 3}$ and $R_{\lambda}=0.9845 \sqrt{\operatorname{Re}_{\text {Grid }}}$ with $\operatorname{Re}_{\text {Grid }}$ as defined in eq. (4.3). We use $\varepsilon=C_{\varepsilon} u_{R M S}^{3} / L$ to estimate $\varepsilon$ using $C_{\varepsilon}=1$ (cf. Sec. 7.1). 

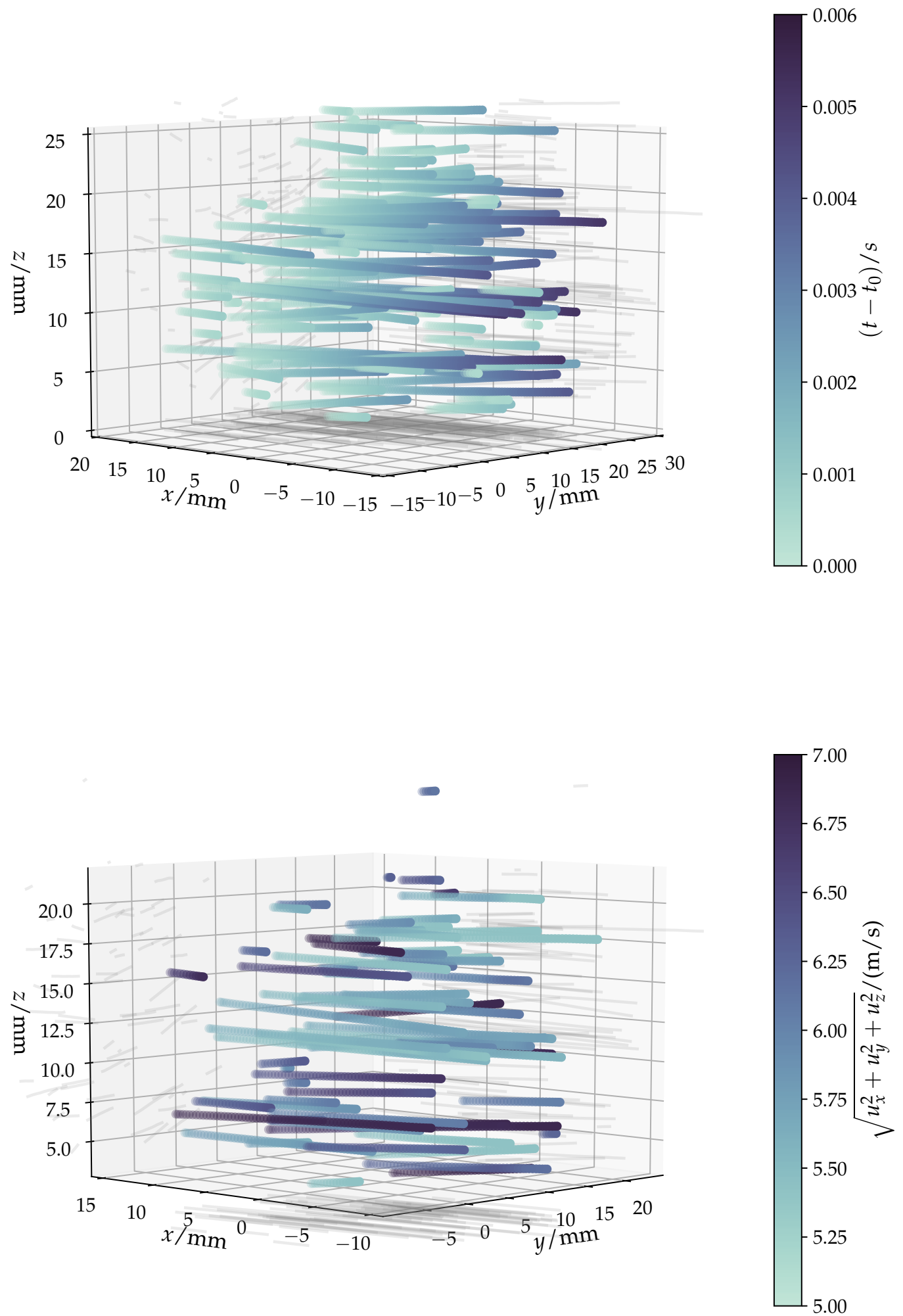

Figure 11.3: Upper panel: Example of particle trajectories with colors indicating time since beginning of the track in seconds. Lower panel: same as upper panel, but with color indicating the the calculated particle velocity in $\mathrm{m} / \mathrm{s}$. 

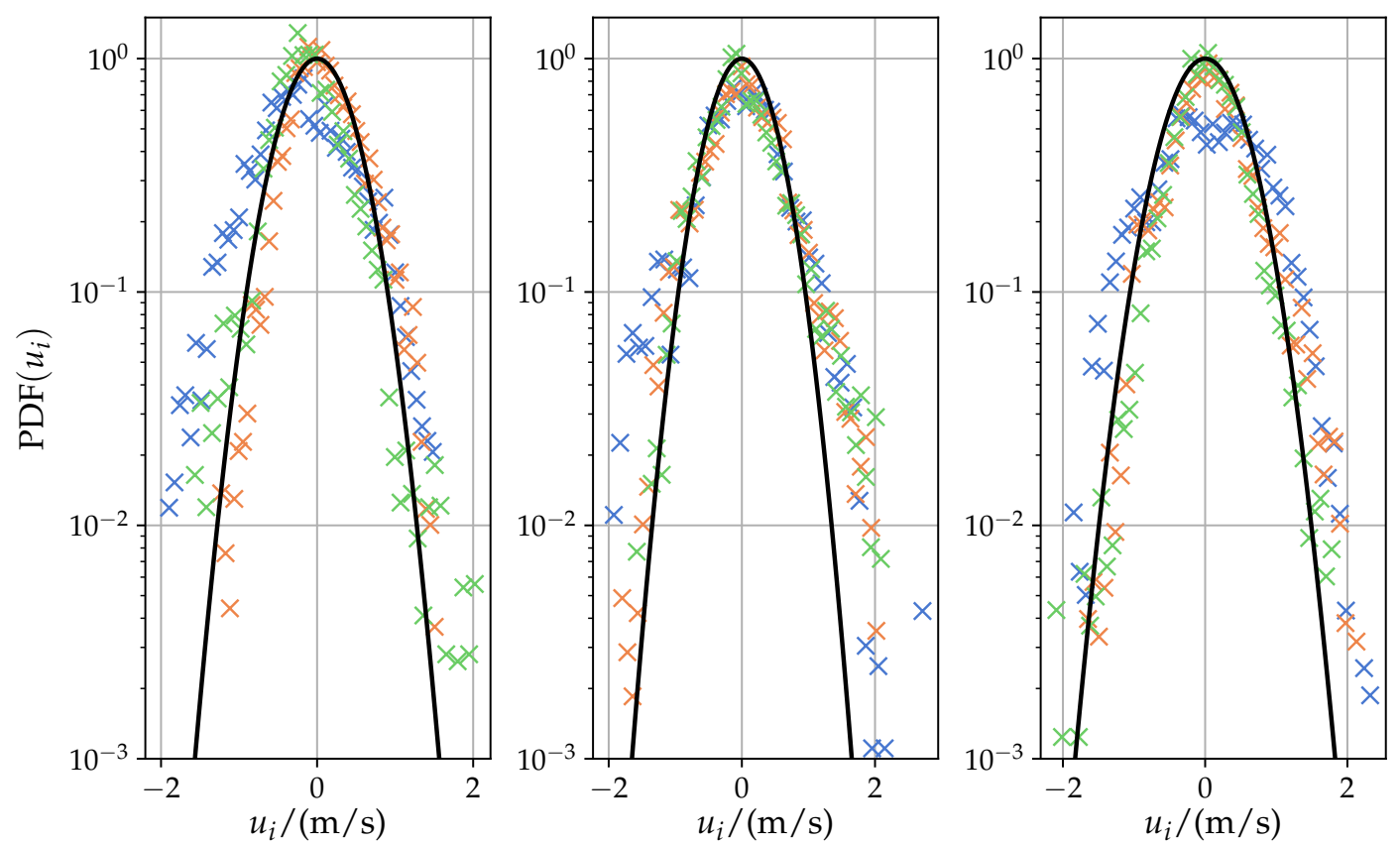

Figure 11.4: PDFs of the velocity for all three experiments with their mean subtracted. Blue markers corresponds to the streamwise velocity, red and green to the spanwise velocity components. Note that the overlap depends on the grid forcing, but only slightly so. The velocity samples were obtained by averaging over all velocities detected within one frame. Black lines are Gaussian distributions with the variance corresponding to that of the streamwise component.

\subsection{Velocity Statistics}

The determination of statistics that typically require a constantly sampled velocity field is more difficult in Lagrangian datasets. The seeding density of the flow is a function of time throughout the duration of one acquisition. For large-scale quantities, such as the RMS of the velocity fluctuations, this can be circumvented by studying frame-by-frame averages. If the field of view is smaller than one integral scale, such averages can be regarded as rough estimates of the true fluctuation intensities and the bias in the velocity PDFs is reduced. Fig. 11.4 shows that the velocities measured this way are have a similar distribution as those obtained from classic hot wire measurements (cf. Fig. 4.7). Most importantly, the differences between streamwise ( $x$-direction) and spanwise ( $y / z$-directions) component of the velocity fluctuations are apparent, but no dramatic anisotropies can be observed in this plot. We estimate the large-scale anisotropy by relating the standard deviations $\sigma$ of the velocity fluctuations in $x$ - and $y$-directions and arrive at $\sigma\left(u_{x}\right) / \sigma\left(u_{y}\right)=1.4,1.1$, and 1.6 for experiments 1,2 , and 3 , respectively. The spanwise velocity components show small non-zero means $(<0.33 \mathrm{~m} / \mathrm{s})$ and the $u_{x}$-distribution of 
Experiment 3 shows a flattened peak. We attribute these unexpected effects to large-scale intermittency and expect them to disappear with increasing number of samples. Equally, the Lagrangian velocity statistics, i.e. the distribution of all velocities found within the particle tracks requires a larger sample size and special weighting procedures to remove the effects of large-scale intermittency and preferential sampling of slower particles. Another possible reason for a small, but finite mean velocity in the spanwise directions is a potential misalignment between the mean flow direction and the calibration mask, i.e. the $x$-component of the data is not parallel to the streamwise direction. Of course, this has no influence on the interpretation of the data since this would correspond to a simple Galilei transformation.

The statistics of the inter-particle distance reveal that the particles very rarely get close to each other. In particular, distances on the order of the Kolmogorov length are generally not observed. The $\eta^{2}$-term in the equation for the Coulomb turbulence number (10.3) can therefore be replaced by a much larger number. Typically, $97 \%$ of particles are more than $200 \eta$ away, such that the maximally allowed particle charge is increased to $\mathcal{O}\left(10^{5}\right)$ to $\mathcal{O}\left(10^{6}\right)$ elementary charges per particle. Such a large charge cannot be expected and electrostatic effects can therefore be neglected in the current setup.

\subsection{Acceleration Statistics of Lagrangian Tracks}

The acceleration of tracer particles probes the highly intermittent small scales of the flow. Consequently, they depend less on the large-scale structure of the flow field and converge towards their presumably universal statistics faster. The data presented here can therefore be used to extract such statistics even though the larger scales are plagued by the small number of large eddies that were sampled. As mentioned above, the velocities were extracted by convolution of the time-resolved particle positions with a two-times differentiated Gaussian kernel of width $w=0.3 \tau_{\eta}$ and a length of $4 w$. The resulting acceleration PDFs are shown in Fig. 11.5 for all three experiments in all three directions. The errors are estimated as proportional to $1.96 \sqrt{N_{c}}$, where $N_{c}$ is the number of counts in each bin.

In addition, the ad-hoc stretched exponential shape for the acceleration suggested by LaPorta et al. [6]

$$
\operatorname{PDF}(a)=C \exp \left(\frac{-a^{2}}{\left(1+|a \beta / \sigma|^{\gamma}\right) \sigma^{2}}\right)
$$



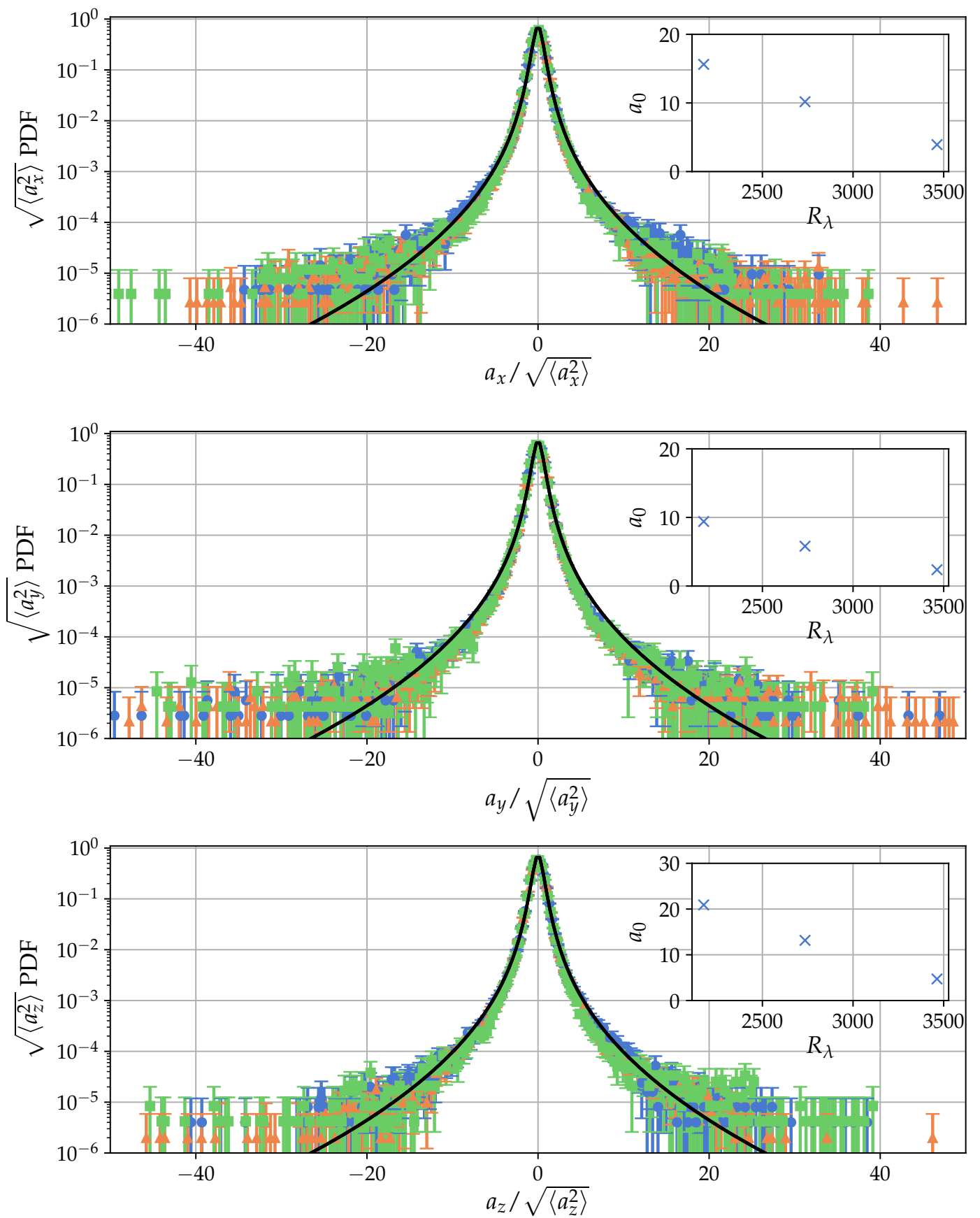

Figure 11.5: PDFs of the acceleration normalised by their standard deviation. Blue circles: experiment $1\left(R_{\lambda}=2176\right)$, orange triangles: Experiment $2\left(R_{\lambda}=2735\right)$, green squares: Experiment $3\left(R_{\lambda}=3463\right)$. Black line: Extended polynomial fit after Refs. $[6,2]$ with parameters obtained by Mordant et al. [23] with a similar differentiation scheme. The insets show the normalised acceleration $a_{0}$ as a function of the Reynolds number for the three directions. 
is shown in each plot using the parameters found by Mordant et al. [23]. This function has proven to describe appropriately normalised acceleration statistics very well $[25,26,27,28]$. We stress that the curves in Fig. 11.5 have not been fitted to the data, but can be seen as a representation of similar curves at lower $R_{\lambda}$.

The normalised PDFs of all three measurements collapse in all three directions to within errors, as well as on the stretched exponential representing earlier data. The PDFs are heavy-tailed and far from Gaussian as expected. The shape of the normalised PDFs appears to be universal.

Fig. 11.5 also shows the normalised acceleration for each direction. Except for the $y$-direction, it is significantly larger than reported in previous studies. It furthermore decreases with Reynolds number, which is also in contrast to earlier measurements [6, 24] and numerical simulations [29, 30, 31].

\subsection{Discussion}

The results presented in this chapter demonstrate that the setup described in Ch. 1o is capable of measuring velocity and acceleration with satisfactory precision when combined with a state-of-the-art particle tracking algorithm. It could be shown for the first time that the anisotropy in the VDTT with the active grid can be varied between at least 1.1 and 1.5, because the particle tracking allows for well-resolved velocity measurements in all three spatial directions. Furthermore, the PDFs of the velocity do not show strong directional dependencies with the exception of the streamwise component of experiment 3. The author stresses however that the basis of these PDFs and the velocity fluctuation intensity is a crude average of all velocities found within a single frame. This is the likely explanation for the somewhat surprising result that a relatively weakly correlated grid protocol produces stronger anisotropy measurements than a stronger correlated grid forcing. It is furthermore expected that a more thorough treatment of the velocity statistics and increased number of samples removes features like that seen in the $x$-direction in Experiment 3 and small mean velocities in the spanwise directions (cf. Fig 11.4).

We have demonstrated that after appropriate treatment of the trajectories, acceleration PDFs can be acquired. They collapse satisfactorily on a stretched exponential with parameters found in earlier studies at lower $R_{\lambda}$ [23]. However, the values of the normalised acceleration variance $a_{0}$ as well as their $R_{\lambda}$ scaling and the apparent anisotropy are far away from the theoretical expectation $[32,33]$ and previous studies. The Gaussian differentiation employed 
for this has recently shown to yield biased estimates of the normalised acceleration variance $a_{0}$ (see eq. (3.35)). Along with the imprecise estimate of $\varepsilon$ and the relatively small number of independent samples, we therefore believe that a measurement of $a_{0}$ requires data conditioning and measurement campaigns that are beyond the scope of this thesis.

It is important to stress that the particles used for this study (KOBO Microspheres D-30) were most likely in the inertial regime with a Stokes number $>1$ and a diameter close to the flow Kolmogorov scale. This was necessary, because the setup did not yield useable recordings if smaller particles with smaller amounts of light were used. It is therefore slightly surprising that the acceleration statistics are still similar to those obtained by tracing particles in Ref. [23]. It shows that more detailed measurements of the Stokes number of the particles is required, e.g. by measuring their settling velocity in a quiescent fluid.

The preliminary study presented here reveals that the setup can be improved substantially by relatively minor adjustments. The amount of light that hit the camera sensors from particles with $\mathrm{St}>\mathbf{I}$ was small and smaller particles with $S t<1$ would yield even lower amounts of light. To increase the amount of light, the laser beam can be narrowed by a factor of 2 . This reduces the requirements on the depth of field of the camera optics allowing us to open the camera aperture and thereby further increasing the amount of light on the sensor pixels.

The observation of the facility during data acquisition revealed that a substantial amount of particles gets trapped within the wind tunnel boundary layers and never reaches the flow center line. Moving the particle injection nozzle towards the centerline of the tunnel is therefore likely to result in a larger amount of trackable particles that is more constant over time.

Despite these caveats, the preliminary data shows that the setup is capable of generating particle tracking data as intended. At least the acquisition of acceleration data and associated quantities such as $a_{0}$ will be possible with the present setup at Reynolds numbers previously out of reach for any Lagrangian experiment. The sampling of Lagrangian structure functions (cf. 3.4.1) in the inertial range will require lower mean flow velocities and larger facility pressures to increase the particle residence time and reduce $\tau_{\eta}$. Extrapolating the results from Fig. 11.2, we estimate that tracks up to $30 \tau_{\eta}$ could be obtained when finely tuning the experimental parameters. 


\section{Bibliography}

[1] C. Tropea, A. L. Yarin, and J. F. Foss, eds. Springer Handbook of Experimental Fluid Mechanics. Berlin, Heidelberg: Springer Berlin Heidelberg, 2007. DOI: 10 .1007/978-3-540-30299-5.

[2] G. A. VOTH et al. "Measurement of particle accelerations in fully developed turbulence". In: J. Fluid Mech. 469 (Oct. 2002), pp. 121-160. DoI: 10.1017/S0022112002001842.

[3] E. W. Saw et al. "Spatial clustering of polydisperse inertial particles in turbulence: II. Comparing simulation with experiment". In: New J. Phys. 14 (2012). DOI: 10.1088/1367-2630/14/10/105031.

[4] G. H. Good and Z. Warhaft. "On the probability distribution function of the velocity field and its derivative in multi-scale turbulence". In: Phys. Fluids 23.9 (Sept. 2011), p. 095106. DoI: 10.1063/1.3632090.

[5] J. Lu, H. Nordsiek, and R. A. Shaw. "Clustering of settling charged particles in turbulence: theory and experiments". In: New J. Phys. 12.12 (Dec. 2010), p. 123030. DOI: 10.1088/1367-2630/12/12/123030.

[6] A. La Porta et al. "Fluid particle accelerations in fully developed turbulence". In: Nature 409.6823 (Feb. 2001), pp. 1017-1019. DOI: 10.1038/ 35059027.

[7] H. Xu, N. T. Ouellette, and E. Bodenschatz. "Multifractal Dimension of Lagrangian Turbulence". In: Phys. Rev. Lett. 96.11 (Mar. 2006), p. 114503. DOI: 10.1103/PhysRevLett.96.114503.

[8] LaVision. DaVis Software for intelligent Imaging. Tech. rep. 2016.

[9] D. Schanz, S. Gesemann, and A. Schröder. "Shake-The-Box: Lagrangian particle tracking at high particle image densities". In: Exp. Fluids (2016). DOI: $10.1007 / \mathrm{s} 00348-016-2157-1$.

[10] N. A. Malik, T. Dracos, and D. A. Papantoniou. "Particle tracking velocimetry in three-dimensional flows - Part II: Particle tracking". In: Exp. Fluids (1993). DOI: 10.1007/BF00223406.

[11] N. T. Ouellette, H. Xu, and E. Bodenschatz. "A quantitative study of three-dimensional Lagrangian particle tracking algorithms". In: Exp. Fluids 40.2 (Feb. 2006), pp. 301-313. DOI: 10.1007/s00348-005-0068-7.

[12] H. Nobach, N. Damaschke, and C. Tropea. "High-precision sub-pixel interpolation in particle image velocimetry image processing". In: Exp. Fluids 39.2 (Aug. 2005), pp. 299-304. DOI: 10.1007/s00348-005-0999-z. 
[13] J. M. St-Arnaud and T. K. Bose. "Direct determination of the intermolecular interaction contribution to the refractive index of carbon dioxide and sulfur hexafluoride". In: J. Chem. Phys. 71.12 (1979), p. 4951. DOI: $10.1063 / 1.438308$.

[14] M. E. Thomas and T. J. Tayag. "Refractive index of He, SF_6, and CO_2 at $063299 \mu \mathrm{m}$ as a function of temperature and pressure". In: Appl. Opt. 27.16 (Aug. 1988), p. 3317. DOI: 10.1364/A0.27.003317.

[15] A. Kubitzek. Deformation of the VDTT under Pressure (private communication).

[16] K. P. Inc. KOBO Microspheres Datasheet. Tech. rep. 2018.

[17] P. Linstrom and W. Mallard, eds. NIST Chemistry WebBook, NIST Standard Reference Database Number 69, Gaithersburg,MD: National Institute of Standards and Technology, 2020. DoI: https://doi .org/10.18434/ T4D30.

[18] S. Garrido-Jurado et al. "Automatic generation and detection of highly reliable fiducial markers under occlusion". In: Pattern Recognit. 47.6 (June 2014), pp. 2280-2292. DOI: 10.1016/j . patcog. 2014.01.005.

[19] G. Bradski. “The OpenCV Library”. In: Dr. Dobb's J. Softw. Tools (2000).

[20] G. An et al. "Charuco Board-Based Omnidirectional Camera Calibration Method". In: Electronics 7.12 (Dec. 2018), p. 421. DOI: 10.3390 / electronics7120421.

[21] Z. Zhang. "A flexible new technique for camera calibration". In: IEEE Trans. Pattern Anal. Mach. Intell. (2000). DOI: 10.1109/34.888718.

[22] K. Muller et al. "Calibration of multiple cameras for large-scale experiments using a freely moving calibration target". In: Exp. Fluids (2020). DOI: $10.1007 / \mathrm{s} 00348-019-2833-z$.

[23] N. Mordant, A. M. Crawford, and E. Bodenschatz. "Experimental Lagrangian acceleration probability density function measurement". In: Phys. D Nonlinear Phenom. 193.1-4 (2004), pp. 245-251. DOI: 10.1016/j . physd.2004.01.041.

[24] J. M. Lawson et al. "Bias in particle tracking acceleration measurement". In: Exp. Fluids (2018). DoI: 10.1007/s00348-018-2622-0.

[25] N. Mordant et al. "Measurement of lagrangian velocity in fully developed turbulence". In: Phys. Rev. Lett. (2001). Dor: 10.1103/PhysRevLett. 87.214501. 
[26] N. Mordant, E. Lévêque, and J.-F. Pinton. “Experimental and numerical study of the Lagrangian dynamics of high Reynolds turbulence". In: New J. Phys. 6 (Sept. 2004), pp. 116-116. DoI: 10.1088/1367-2630/6/1/ 116.

[27] A. M. Reynolds et al. "On the distribution of Lagrangian accelerations in turbulent flows". In: New J. Phys. (2005). DoI: 10.1088/1367-2630/ $7 / 1 / 058$.

[28] R. Shnapp et al. "Extended 3D-PTV for direct measurements of Lagrangian statistics of canopy turbulence in a wind tunnel". In: Sci. Rep. (2019). DOI: 10.1038/s41598-019-43555-2.

[29] J.-I. Choi, K. Yeo, and C. Lee. "Lagrangian statistics in turbulent channel flow". In: Phys. Fluids 16.3 (Mar. 2004), pp. 779-793. Dor: 10.1063/ 1.1644576 .

[30] P. K. Yeung and S. B. Pope. "Lagrangian statistics from direct numerical simulations of isotropic turbulence". In: J. Fluid Mech. 207 (Oct. 1989), pp. 531-586. DOI: 10.1017/S0022112089002697.

[31] D. Buaria. "Lagrangian Investigations of Turbulent Dispersion and Mixing Using Petascale Computing". PhD thesis. Georgia Institute of Technology, 2016.

[32] A. Yaglom. "On the local structure of a temperature field in a turbulent flow". In: Dokl. Akad. Nauk. SSSR (1949).

[33] W. Heisenberg. "Zur statistischen Theorie der Turbulenz". In: Zeitschrift für Phys. 124.7-12 (July 1948), pp. 628-657. DOI: 10.1007/BF01668899. 


\section{Part IV}

\section{Discussion and Outlook}





\section{Chapter 12}

\section{Discussion}

Turbulent fluid motion is widely regarded as the "most important unsolved problem of classical physics" (Richard Feynman). While the corresponding equations of motion can be straightforwardly formulated (see Sec. 2.1), their nonlinear and non-local nature makes analytical treatments challenging. Their numerical integration bears extreme computational costs if the fundamentals of turbulent flows are to be studied. Statistical theories regularly end in closure problems, which require the ad-hoc assumption of closure models (see. Sec. 3.2). The turbulence problem could therefore be described as a quest for an analytically tractable, or numerically cheap theory that accurately describes the process through which the coherent fluid motion at very large scales is transported towards a regime where viscosity transforms the turbulent kinetic energy into unordered heat. Such a theory appears out of reach at the point of writing and it is unlikely that this will change soon. Such a hypothetical model would have to answer - among many others - the following questions: Does a regime exist at finite $R_{\lambda}$, where the flow is dominated by inertial effects, and flow geometry and viscosity have a negligible impact? How do the inertial motions organise the scale-to-scale energy transfer? How universal is this process, i.e. how much does it vary from flow to flow? The extent to which the thesis at hand provides insights towards those questions shall be discussed in the following paragraphs.

\subsection{Large Reynolds Numbers in the VDTT}

This thesis approaches the aforementioned key questions purely from an experimentalist's point of view. The facility of choice is the Variable Density Turbulence Tunnel [1] as described in Ch. 4, since it is uniquely suited 


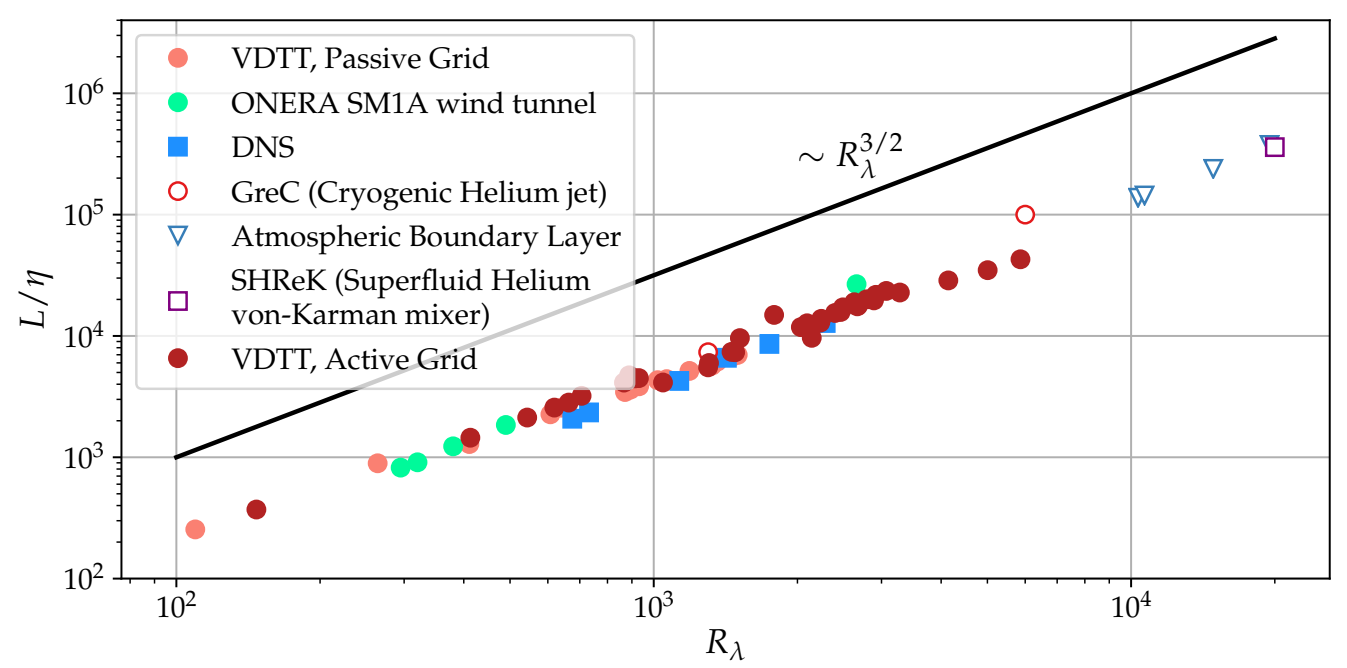

Figure 12.1: Separation of scales as a function of $R_{\lambda}$ for selected high Reynolds number flows (same as Fig. 1.1, with the active grid data added). The range of $R_{\lambda}$, where data at approximately homogeneous and isotropic turbulence exists (indicated by closed symbols) is augmented by a factor of more than 3 .

to study (approximately) homogeneous and isotropic turbulence at high $R_{\lambda}$. This ability has been demonstrated mainly in $\mathrm{Ch}$. 4. In particular, the range of Reynolds numbers obtainable in the VDTT is increased by a factor of more than 4 compared to the same setup with only a passive grid (see [2]). Fig. 12.1 shows that the installation of an active grid has opened a new regime of $R_{\lambda}$ that can be studied systematically under approximately homogeneous and isotropic conditions. Since the separation of viscous and inertial length scales holds the promise to reveal some of the underlying dynamics of turbulence (see Ch. 3 and references therein), this is an important advancement in the topic.

The homogeneity of the flow in the Variable Density Turbulence Tunnel with an active grid installed was explored in $\mathrm{Ch}$. 4. It was found that the region of negligible shear is smaller than in the case of the passive grid [1], but can be expanded substantially by limiting the solidity of the boundary flaps. Furthermore, the size of the shear-free region and therefore the homogeneity of the flow depends on the grid motions chosen. It thus allows tailoring the flow towards homogeneous, and isotropic conditions (see below). The active grid motions are well-characterised by the grid's mean solidity, and its spatial and temporal correlation lengths. Larger mean solidities and correlations lead to larger integral scales and turbulence intensities, but a smaller shear-free region. There is therefore a compromise between the size of the homogeneous region and the Reynolds number of the flow. 
This manifests itself also in one-point statistics of the velocity fluctuations, which depart from Gaussian statistics if intense correlations are introduced into the flow by the active grid (see Figs. 4.7 and 4.8). It is not trivial to what extent these large-scale phenomena influence the statistics of the inertial and dissipative scales $[3,4,5]$. Since double- or triple wires at sufficient spatial resolution have not been realised to date, it is difficult to even measure the anisotropy at small scales in the VDTT. Measurements in a slightly higher, but otherwise identical measurement section under air have shown that the active grid can introduce substantial anisotropy if the spatial and temporal correlation lengths are mismatched drastically [6]. This is in agreement with the results presented in $\mathrm{Ch}$. 4 . In this regard, the particle tracking system presented in Part III is an important progress, because it allows the measurement of all three velocity components and thus an assessment of the flow anisotropy. Preliminary measurements (see Sec. 11.2) suggest that the anisotropy varies approximately as predicted in the air wind tunnel [6]. This means that the data presented here contains flows close to homogeneous, isotropic turbulence as well as anisotropic flows. The thesis only makes statements that are robust against changes in the active grid protocol, i.e. where these effects do not influence the statistics.

These experimental challenges can be approached more rigorously by utilising the unique flexibility of the facility itself. The flow at a given $R_{\lambda}$ can be recreated at various different length scales. For this, active grid and facility pressure are adjusted accordingly. This makes the facility ideally suited to explore the universality of the results across different flows. Furthermore, the resolution of the instrumentation can be tested in the spirit of Ref [7], and measurement biases excluded, since the same Reynolds number can be reproduced with different instrumentation requirements (usually given by the Kolmogorov time- and length scales).

This thesis presents a large database of turbulent time series that contains flows over a wide range of experimental parameters and Reynolds numbers. Observations that hold across these measurements are therefore robust against substantial changes in the large-scale flow structure, i.e. large-scale inhomogeneities and anisotropies. They are further shown to be independent of the relative sensor resolution. These arguments fail at the limit of the facility, where all experimental parameters have to be optimised to reach $R_{\lambda}>3500$. The results further cannot be carried over to entirely different flow geometries, such as a von-Karman mixer.

During the course of this thesis the influence of the active grid motions 
on the downstream turbulence properties were systematised through the introduction of a correlation volume and a related grid Reynolds number. This allows an a priori estimate of the Taylor-scale Reynolds number a set of experimental parameters will yield.

To summarise, the VDTT can be regarded as the most advanced facility to study turbulence at large $R_{\lambda}$. Its flow is closest to the canonical ideal of homogeneous isotropic turbulence at $R_{\lambda}>2500$ worldwide. The ability to control three different parameters independently (active grid, wind speed, and facility pressure) is equally unmatched at the point of writing. It allows the exploration of the robustness of turbulence statistics against different degrees of anisotropy and inhomogeneity. This is particularly important when making statements about the universality of the results.

\subsection{High-Resolution Measurements of Turbulence}

The fully resolved measurement of flow velocities at pressurised facilities such as the VDTT is challenging because the flow structures are particularly small. The majority of the data presented in this thesis were sampled using a subminiature hot wire (NSTAP, see Ch. 5 and references therein). Even though the NSTAP represents the state-of-the-art at the point of writing, it cannot resolve the smallest Kolmogorov scales $\eta<10 \mu \mathrm{m}$ present in the VDTT at the highest Reynolds numbers. Furthermore, the attached constant temperature circuitry proved to introduce frequency-dependent biases [7]. Finally, the probe boundary layer might play a significant role where small velocity fluctuations need to be sampled with a high temporal resolution. Those probe effects were analysed for the given setup. It was found that the temporal and spatial resolution introduces errors on second-order statistics such as the turbulence dissipation rate of less than $10 \%$. The probe boundary layer was found to be of minor importance for all but the highest $R_{\lambda}$, where small effects might influence the statistics at the smallest scales.

The frequency response of the hot wire system was investigated in detail. The previous findings of Hutchins et al. [7] that the frequency response of the used hot wire system slightly attenuates the velocity signal at relatively small frequencies were confirmed. Recent progress in micromachining processes allowed the production of novel subminiature hot wires (Twente Hot Wire) that potentially resolve several shortcomings of the NSTAP [8] (see Appendix $C$ for a reprint). In particular, the frequency response of this new wire was tested against the NSTAP. It was found that the wire's thermal time con- 
stant limits the temporal resolution of both wires to around $1 \mathrm{kHz}$, which is insufficient for measurements of small scale turbulence. Interestingly, when operated with a constant current anemometer, the NSTAP, which has a rectangular cross-section, shows a slight roll-off starting around $100 \mathrm{~Hz}$ (see Fig. 5.6). A similar behaviour can be observed when they are operated in constant temperature mode, albeit at much larger frequencies. The Twente Hot Wire, whose cross-section is almost quadratic (see Appendix C and [8]), does not show this roll-off. One might therefore speculate that the shape of the wire significantly influences the aerodynamics around the probe and hence its response at small frequencies.

A strategy was developed to investigate the $R_{\lambda}$-dependence of extremely small structures present at large wavenumbers, where the system's frequency response cannot be assumed constant. It makes use of the active grid's ability to change the Reynolds number at constant wind tunnel pressure and mean flow speed. Because the frequency at which a spectral feature occurs remains the same for different $R_{\lambda}$, the biases in the measured feature due to a variable frequency response also remain the same. The applicability of this method is limited to flows with a well-controlled, flexible turbulence production mechanism, such as active grids or jet arrays. In principle, the method should also be helpful in exploring questions of scaling to some degree, but this remains to be shown in practice. To the author's knowledge, no other method exists that allows the extraction of the $R_{\lambda}$-scaling of very small spectral features under unknown instrument frequency responses.

An important aspect of turbulent flows, especially with regards to engineering applications, is their excellent mixing efficiency and transport properties. These phenomena are best studied by shifting the point of view away from one- or two-point measurements of the flow towards the motion of individual fluid elements over time, i.e. the Lagrangian framework. When studying turbulence in this way, even larger Reynolds numbers (by a factor of $R_{\lambda}^{1 / 2}$ [14]) compared to the Eulerian picture are required to observe an inertial scaling range. Therefore, facilities such as the VDTT are even more important to gain knowledge about Lagrangian turbulence.

Lagrangian measurements typically rely on tracer particles that follow a single fluid element without significantly influencing the flow. These measurements are extremely challenging and became available in turbulent flows only around the turn of the century through the pioneering works of Virant, Dracos, Mordant, Bodenschatz, and their respective coworkers (see [14] for a review). Since the extraction of Lagrangian quantities from DNS is also 


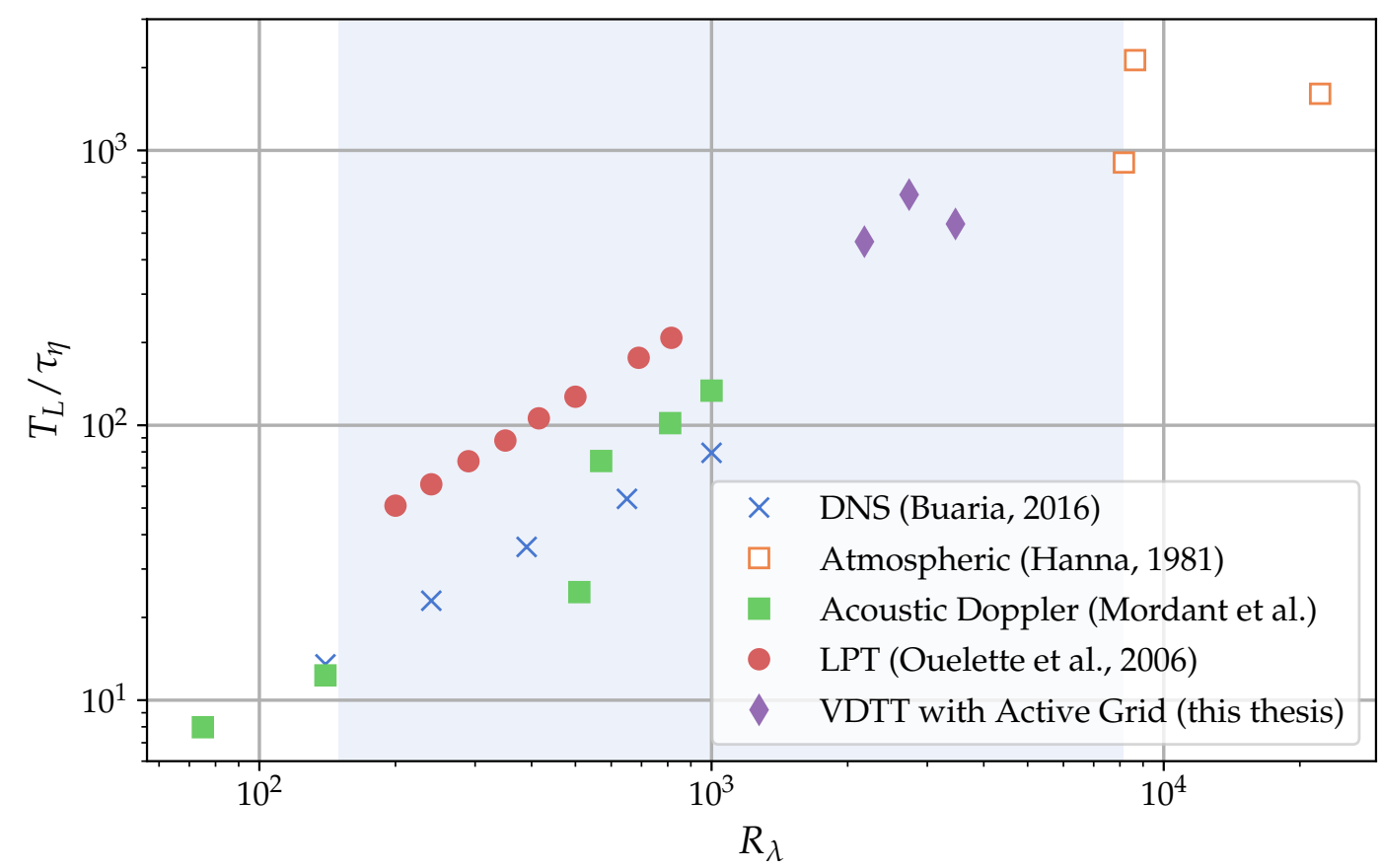

Figure 12.2: Separation of large-eddy and viscous time scales vs Reynolds number for a selection of Lagrangian measurements and numerical simulations. The shaded area indicates the region of $R_{\lambda}$ that can be explored with the LPT system in the VDTT with the data presented in Ch. 11. DNS data from Ref. [9], Acoustic Doppler data from [10, 11], atmospheric measurements with "tracer" balloons [12], LPT measurements using high speed cameras [13].

challenging $[15,16]$, Lagrangian datasets are very scarce. In particular, measurements at high $R_{\lambda}$ require such high sampling frequencies (imaging frame rates) that they are notoriously limited by the available camera technology.

In the VDTT, the presence of a mean flow and Kolmogorov length scales of less than $10 \mu \mathrm{m}$ and time scales of faster than $1 \mathrm{~ms}$ complicate Lagrangian measurements further. For this reason, the Lagrangian setup presented in Pt. III is a leap forward in terms of measurement technology. To the author's knowledge Lagrangian particle tracking has not been demonstrated in a pressurised environment before. The Reynolds numbers at which Lagrangian quantities are accessible have definitely been tripled, most likely increased by almost a decade. Finally, the particle tracking system complements the single-directional hot wire measurements allowing time-resolved Eulerian measurements in all three spatial directions provided a large seeding density and suitable analysis techniques. 


\subsection{Inertial Range Universality}

The question, whether the inertial range statistics of homogeneous isotropic turbulence are independent of the flow geometry at very large $R_{\lambda}$ has concerned researchers for almost a century. Universality was first postulated by Kolmogorov in 1941 [17] and the corresponding scaling laws were refined subsequently. Until now, the Reynolds numbers where such statistics could be measured systematically in well-controlled environments were too low to exclude effects of flow geometry or viscosity on the inertial range [18, 19], subject to extreme anisotropies [20], or lack of suitable instrumentation [21, 22]. This thesis represents progress on this matter, because inertial range statistics can be observed over a wide range of scales (up to three decades) and certain statistics do not change any longer with increasing $R_{\lambda}$.

The findings are best discussed in terms of the frequently used decomposition

$$
S_{n}(r)=C_{n}(\varepsilon r)^{n / 3} f_{n}(r / \eta),
$$

where $f_{n}$ is a positive, concave function and $C_{n}$ depends on flow geometry and Reynolds number.

The Kolmogorov theory predicts scaling of structure functions in the inertial range of $S_{n} \sim(\varepsilon r)^{n / 3}$. The precise value of the exponent is almost certainly influenced by intermittency and therefore different from $n / 3$. One way to investigate the structure function scaling is to study the local scaling exponent defined as $\zeta_{n}(r)=d \log \left(S_{n}\right) / d \log (r)$ as described in detail in Ch. 6 . The structure function shape measured in this way becomes independent of $R_{\lambda}$ in the inertial range if the Reynolds number exceeds approximately 2000. This holds up to order $n=6$. In fact, the $\zeta_{n}(r)$ collapse even within the dissipation range and differ only slightly up to $r \approx 0.1 \mathrm{~L}$. The author concludes that $f_{n}$ is independent of $R_{\lambda}$ in this regime.

An exception are the three largest $R_{\lambda}$ above 4000, where both the active grid and the measurement instruments were operated at their limits, which likely explains the deviations that are visible for $n>2$.

In addition to the $R_{\lambda}$-independence we find that the structure-function shapes are robust against substantial changes in the active grid protocol. However, when investigating the nondimensionalised structure functions themselves, i.e. $S_{n} /(\varepsilon r)^{n / 3}$, a significant $R_{\lambda}$-dependence as well as substantial scatter remains (see Figs. 7.3 and 7.2). Therefore, $f_{n}$ is relatively independent of the turbulence generation, whereas $C_{n}$ is sensitive towards it, also when considering measurement errors of $\varepsilon$. The latter observation is in agreement 


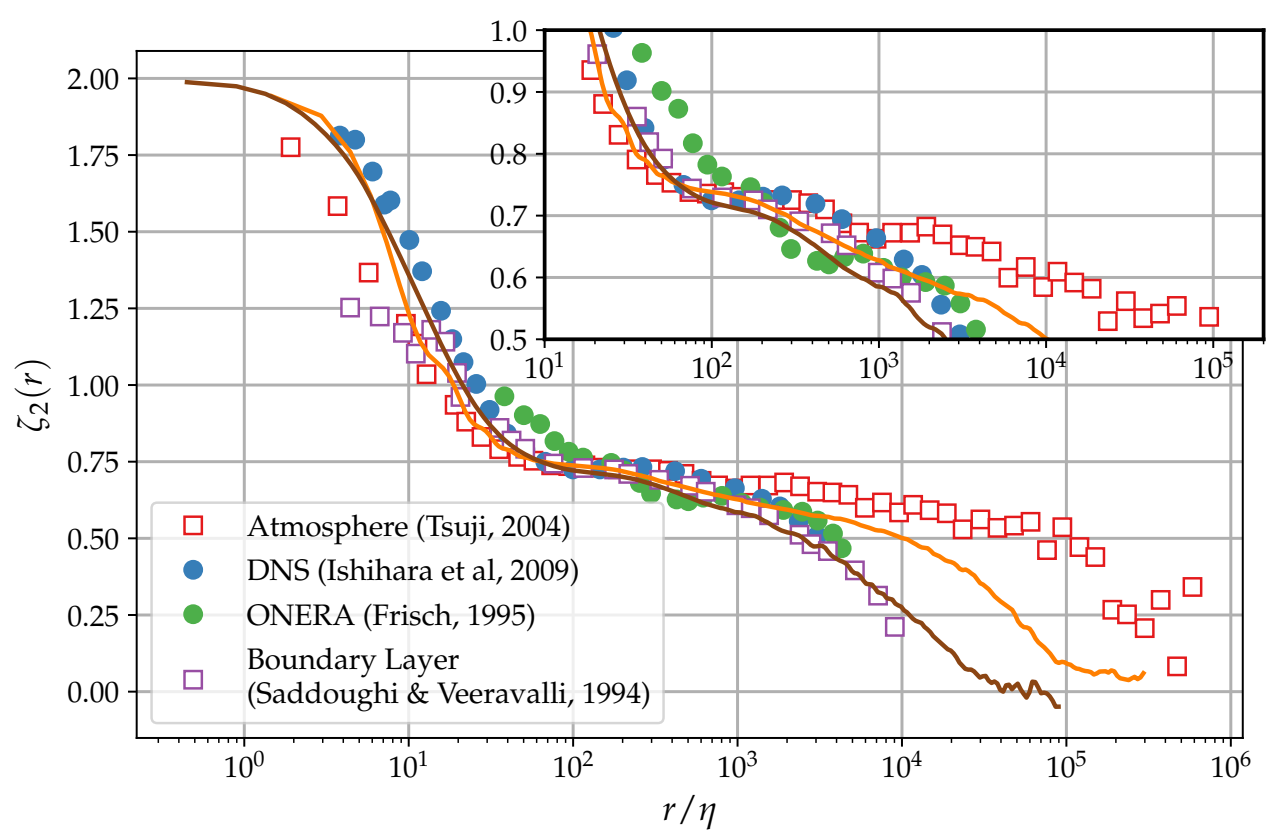

Figure 12.3: Local slope of $S_{2}(r)$ for different flows compared to active grid data at $R_{\lambda}=1480$ (brown) and at $R_{\lambda}=5006$ (orange). Atmospheric data $\left(R_{\lambda}=17060\right)$ from Ref. [29], all other data taken from compilation in Ref. [25]: DNS from Ref. [30] $\left(R_{\lambda}=1131\right)$, ONERA data from [31] $\left(R_{\lambda}=2660\right)$, boundary layer from Ref. [32] $\left(R_{\lambda}=1450\right)$. Inset: Same data, zoomed into the inertial range.

with previous studies at lower $R_{\lambda}[23,24,25,26]$.

The universality of $f_{n}$ (measured by $\zeta_{n}(r)$ ) only comes to effect when $R_{\lambda}>$ 2000 , and therefore comparing studies are scarce. However, even at lower $R_{\lambda}$ it is clear that the local slopes in numerical simulations are fundamentally different $[27,28]$. We offer an explanation for these differences in Ch. 6 in terms of the flow instationarity. Antonia et al. [25] assembled various $\zeta_{2}(r)$ and $\zeta_{3}(r)$ from different flows that are roughly similar to those found in $\mathrm{Ch}$. 6 as shown in Fig. 12.3. All laboratory experiments agree well given their different geometries. The atmospheric data shows a similar trend in that no clear region where $\zeta_{2}=$ const. can be identified. However, the inertial range appears flatter than in the wind tunnel data. The DNS data shows a clear inertial range plateau in contrast to the experimental data.

The author infers that the shape of $S_{2}$ is universal within a given class of flows at large $R_{\lambda}$. If $R_{\lambda}$ is large enough, even substantial changes of the active grid forcing have little effect on the shape of $S_{2}$. However, moving towards continuously forced turbulence such as in most DNS or the stratified, inhomogeneous, multiphase flow of the atmosphere changes the shape of $S_{2}$. In the light of these results, the author further raises the question whether existing departures from self-similar scaling laws should still be regarded as 
"finite Reynolds number effects", since they appear to influence the entire energy cascade independent of $R_{\lambda}$. Finally, the author advocates a separate treatment and understanding of $S_{2} /(\varepsilon r)^{2 / 3}$ and $\zeta_{2}(r)=d \log \left(S_{2}\right) / d \log (r)$, since the former is much more sensitive towards changes in the turbulence forcing.

Under these considerations, eq. (12.1) could be refined as

$$
S_{n}(r)=C_{n}\left(R_{\lambda}, \mathrm{TF}\right)(\varepsilon r)^{n / 3} f_{1}(r / \eta, n) f_{2}\left(r / \eta, R_{\lambda}\right)
$$

$f_{1,2}$ depend on the broad category of turbulent flows and $f_{2}$ decays with $R_{\lambda}$ relatively fast. TF denotes a (small) dependence on the details of the energy injection and varies from flow to flow. Intermittency corrections to the scaling functions would appear as $f_{1}(r / \eta, n)=(r / \eta)^{\zeta_{n}-n / 3} \tilde{f}_{1}(r / \eta)$, where $\zeta_{n}$ is the intermittency-corrected scaling exponent.

In physical terms, this suggests that even at the large $R_{\lambda}$ presented in this thesis the nonlinear transfer mechanisms of the energy cascade do not decouple completely from the way they are initiated. For example, the difference between the data presented here and DNS at large $R_{\lambda}$ could be partially explained by comparing decaying and continuously forced turbulence in a self-similar model of decaying turbulence. This explains why scaling laws are more easily observed when considering $S_{n}$ vs $S_{|3|}$ (ESS) [33]. The geometryand $R_{\lambda}$-dependence vanish and the underlying, presumably universal structure is revealed. Accordingly, attempts to extract scaling exponents using a model for $f_{1}$ (Ch. 6) yield scaling exponents that are in good agreement with those calculated from ESS (Sec. 7·3).

A similar, though more subtle effect can be observed at dissipative scales (see Ch. 8). When removing the general inertial range trend within $\zeta_{n}(r)$, a fine structure becomes apparent that is well-approximated by log-periodic oscillations (Fig. 8.3). These oscillations are reminiscent of the structure Sinhuber et al. [34] observed in $d \log \left(S_{n}\right) / d \log \left(S_{m}\right)$, even though systematic differences exist. As they point out, this fine structure is linked to the dissipation range, since it collapses upon normalisation by the Kolmogorov scale $\eta$. However, it persists throughout the entire inertial range. The extended inertial range allowed the detailed analysis of these oscillations over a wide range of $R_{\lambda}$. Earlier studies such as Ref. [35] also identify oscillating features in structure functions, but do not explore them in detail.

The physical origin of this fine structure is unclear. It was shown in this thesis that the frequency of the log-periodic oscillations is too low to be ex- 
plained by the breakup of eddies into a small number of daughter eddies only, as suggested by Novikov [36]. Furthermore, it would be plausible that the collapse happens upon normalisation at larger scales in this scenario. Even though the underlying physical mechanisms have to be explored further, the data supports some sort of discrete scale invariance [37].

The results are somewhat reminiscent of intermittency models that construct a hierarchy of moments based on the fractal dimension of the most dissipative structures $[38,39,40]$ that ultimately dictates the inertial range scaling exponents. However, this connection is circumstantial at best and further study of the origin of the log-periodic oscillations is necessary. The important observation is that dissipative effects, as well as certain properties of the large-scales, influence the statistics throughout the entire inertial range and show no sign of reduction with $R_{\lambda}$.

\subsection{Towards Statistics of the Small Scales}

The dissipation range, where the dynamics are governed by the transfer of structured fluid motion into heat is at the heart of fundamental questions in turbulence. The reason is that the symmetry breaking by viscosity forces the steep "ramps" in the velocity signal that ultimately lend shape to the fractal characteristics of turbulence. Consequently, the small scales also contain the flow structures of strongest intermittency, namely vortex filaments. Even though the small scales are the least anisotropic and thus less sensitive to varying large-scale anisotropy, they are the most challenging to measure experimentally. This thesis demonstrates progress in the measurement of extremely small turbulent scales.

The velocity field at the smallest scales needs to accommodate for whatever turbulent power is delivered by the energy cascade. The "coupling constant" between the velocity field and heat is the viscosity. As the viscosity becomes smaller, the velocity field forms sharper and sharper gradients. It is fundamental in the understanding of turbulence [41] as well as the NavierStokes equations in a mathematical sense [42], whether singularities in the field of velocity gradients arise at large, but finite Reynolds numbers. One indication of this is the constancy of the normalised dissipation rate $C_{\varepsilon}=u^{3} \varepsilon / L$. While the value of $C_{\varepsilon}$ depends on the overall structure of the flow (see [43] for a recent review), its $R_{\lambda}$-scaling provides information about the presence of singularities, since $\varepsilon \sim v(\partial u / \partial x)^{2}$. The data presented in Sec. 7.1 show that for our scan of almost one decade in $R_{\lambda}>800$, no substantial departure 
from $C_{\varepsilon}=$ const. could be found. The data is therefore not inconsistent with the rise of singularities in the limit $R_{\lambda} \rightarrow \infty$. These results extend the existing body of literature on $C_{\varepsilon}$ by systematically gathered data with a larger $R_{\lambda}$ in a laboratory and are in overall agreement with previous studies. The author knows of no wind tunnel study that has measured $C_{\varepsilon}$ at larger Reynolds numbers. Risius et al. [44] measured $C_{\varepsilon}$ on a mountain research station at $R_{\lambda}$ up to 5000 and equally find an independence of $R_{\lambda}$, although their measured value of $C_{\varepsilon}$ is smaller.

Another effect linked to the dissipation range of scales is the bottleneck effect, i.e. the prominent bump in the compensated energy spectrum at the transition from the inertial to the dissipation range. The origin of this bump has been the subject of several theories outlined in Ch. 9. Furthermore, it has been shown in DNS that the intensity of the maximum diminishes with increasing Reynolds number. In this thesis, it was shown for the first time systematically in an experiment that the bottleneck effect gets smaller with increasing $R_{\lambda}$ up to $R_{\lambda}<4000$. A good agreement with the DNS results [24] as well as a shell model [45] was found. However, the absolute height of the bottleneck could not be quantified as a function of $R_{\lambda}$. Furthermore, the experimental errors at the largest $R_{\lambda}$ were too large to conclude whether the bottleneck effect saturates at these large Reynolds numbers.

Important aspects of the velocity derivative statistics could not be approached with the current dataset. The skewness and flatness of the velocity derivatives are important quantities characterising the inertial range nonlinearity and the small-scale intermittency, respectively. Showing their approach towards a constant value at finite $R_{\lambda}$ would be an essential case in favour of $\mathrm{K}_{41}$. The hot wire data at the smallest scales failed to provide enough resolution to measure these quantities reliably. However, the particle tracking system is capable of measuring such small-scale quantities in the Lagrangian frame of reference. This capability has been demonstrated and will be exploited in the future. Preliminary results on Lagrangian small-scale properties reveal results that are only partially in accordance with the existing body of literature. The data acquisition and analysis need to be improved to yield more reliable results. The following chapter will elaborate on the studies that will be possible once these improvements have been accomplished. 


\section{Chapter 13}

\section{Outlook}

In terms of fundamental insights into turbulence, this thesis' main findings are concerned with the transition from a regime where the inertial range statistics strongly depend on Reynolds number towards a regime when they are at least partially $R_{\lambda}$-independent. A surprising finding is that viscosity and flow geometry still influence the inertial range throughout its entire extent. The author believes that it is important to trace back these statistical imprints of large-scale effects to specific physical processes. While models, such as the one by Yang et al. [48] used extensively in this thesis, are very helpful in finding the possible origin of a given observation, numerical simulations provide a level of detail that allows for much deeper insight. For this, the experimental configuration should be more closely resembled in numerical setups even at the expense of Reynolds number. For example, identifying the structural differences between a forced and a decaying flow could provide important physical insights even at modest $R_{\lambda}$. Machine Learning technologies might be able to reveal geometric differences between such flows following ideas such as the structure identification of Leung et al. [47].

In general, the author argues based on the results of this thesis that deviations from canonical strictly homogeneous and isotropic turbulence should be considered as potentially insightful into the principles that govern the floworganisation instead of undesirable finite-Reynolds-number effects. Even when the statistics are independent of $R_{\lambda}$ in the inertial range, the large-scale structure and dissipative effects cannot be neglected. Therefore, even when $R_{\lambda}$ is extremely large, any model accurate for perfectly homogeneous and isotropic turbulence might provide poor descriptions of real-world flows that usually carry at least small anisotropies and inhomogeneities. The limit of $R_{\lambda} \rightarrow \infty$ might turn out to be a misleading theoretical simplification.

The interdependence of scales and the successful description of data when 
using models, which consider the experimental reality should motivate efforts to find models such as the generalised self-similar model by Yang et al. [48] also for strong anisotropies, stratification and other common large-scale effects.

The fine-structure in the dissipation range discussed in Ch. 8 equally spans many scales. Its presence in DNS is still subject to ongoing research [49]. It would be of great interest to revisit existing theories on discrete scaleinvariance in the light of the results presented here and elsewhere [34]. The author speculates that the termination of the cascade at the dissipation scale causes some scales to be preferred, while others are slightly suppressed. As in the case of the bottleneck effect [50, 45], shell models can be of help in disentangling the scale dynamics that cause this oscillating fine structure.

The experiment presented here could contribute further to the matter through time series whose statistical fidelity approaches those of Ref. [34] at larger $R_{\lambda}$. Due to the larger integral scales involved in the active grid case, about one week of continuous data acquisition would be required and experimental conditions should thus be chosen carefully. This would also allow the study of higher order structure functions $S_{n}$. The data presented here points towards a gradual flattening of the local exponent as $n>6$ in the inertial range, but the statistical uncertainties prevent conclusive statements.

Ideally, such measurements would be performed with an improved hotwire system. Even though imaging methods become easier to operate and afford and provide much more information, hot wires remain the method of choice when good statistical convergence is required and one-dimensional information is sufficient or particle seeding is not an option. For precision measurements in flows of low kinematic viscosity (and therefore small $\eta$ ), subminiature wires developed only during the last decade are necessary. Their operation with CTAs is still a practical challenge, because the feedback loops of commercial CTAs are designed for standard wires and frequently cause structural damage to subminiature wires. The author believes that the development of a constant voltage anemometer (CVA) is a path towards a setup, where the operating point of the subminiature wire can be finely adjusted, while maintaining a constant response at very high frequencies.

Subminiature wires themselves are still a matter of active development, where the Twente Wire presented in Ref. [8] (see Appendix C) is the most recent addition. It will be important to verify and explain the discrepancies in frequency response indicated in Fig. 5.4. One possible line of thinking would be that the aerodynamics of the flat NSTAP cause a reduction in the frequency 
response.

The Lagrangian particle tracking system introduced in this thesis will spark a series of groundbreaking experiments. While the Reynolds numbers found in the VDTT are not entirely unseen in the Eulerian reference frame (Atmospheric measurements and experiments in liquid and superfluid helium provide some data), Lagrangian turbulence has never been explored at $R_{\lambda}>1300$ to the author's knowledge. Measurements of the normalised acceleration $a_{0}$ and potentially the inertial range constant $C_{0}$ at high $R_{\lambda}$ are within reach and can be performed within months at the point of writing. Several small adjustments described in Ch. II will make these measurements more reliable than the preliminary results shown in this thesis.

The Lagrangian data will furthermore allow experimental tests of recent theoretical results regarding a correspondence between Eulerian and Lagrangian statistics [51] and a hierarchy of Gaussian statistics based on the coarsegrained acceleration [52].

Closely connected to the Lagrangian inertial range constant $C_{0}$ is the constant that characterises the Richardson-Obhukov regime of two-particle dispersion statistics in the inertial range. Namely, the distance $|\mathbf{r}|$ between two initially close particles is expected to grow with time $t$ as $|\mathbf{r}|=g \varepsilon^{3}$ with a universal constant $g$ if the value of $t$ exceeds the Batchelor time (see reviews $[53,14]$, as well as [54] for details). To observe this behaviour, tracks of length $30-100 \tau_{\eta}$ would be required. Furthermore, a decade of $t^{3}$-scaling is expected only at $R_{\lambda}>6000[13,55]$, which explains why the extensive study of this regime has remained elusive. The VDTT is capable of achieving large enough $R_{\lambda}$, but tracking particles for such a long time is difficult due to the presence of a mean flow. One way to circumvent this is to move the image acquisition system with the mean flow. This is however a major technological challenge even though the existing particle tracking setup can be built upon. The author estimates that such an endeavour would prevent measurements in the facility for more than one year. A relatively quick alternative was already mentioned in Sec. 10.1.3. If the camera resolution and sensitivity are large enough, a larger measurement volume can be allowed for. Naturally, a large number of pixels reduces the frame rate of the camera. The author therefore suggests to acquire long exposure photographs instead of high speed videos using a camera of suitable resolution. The moving particles would appear as bright lines on the images. The time information that is lost can be partially recovered by modulating the illumination intensity and thereby the lines' brightness on the camera sensors over time. Using two $100 \mathrm{Mpx}$ camera, such as the Phase One 
XF iXM, a $3 \mathrm{D}$ measurement volume can be created, which spans up to $0.5 \mathrm{~m}$ in the streamwise direction allowing particle tracking for $>100 \tau_{\eta}$ at $5 \mathrm{~m} / \mathrm{s}$.

The thesis has shown that around $R_{\lambda} \approx 2000$ the scaling of the inertial range can be clearly distinguished from the dissipation range even though it appears different than expected. We are therefore approaching an era where Eulerian and Lagrangian data at large-enough Reynolds number exists in both experiments and direct numerical simulations. The collaboration between these efforts might not lead to a general "Ising model of turbulence", but will certainly deepen our knowledge of the subject, and perhaps put to rest some long-standing questions. 


\section{Bibliography}

[1] E. Bodenschatz et al. "Variable Density Turbulence Tunnel Facility". In: Rev. Sci. Instrum. 85.9 (2014), pp. 1-45. DOI: 10.1063/1.4896138.

[2] M. Sinhuber. "On the Scales of Turbulent Motion at High Reynolds Numbers". PhD thesis. Georg-August-Universität Göttingen, 2015.

[3] X. Shen and Z. Warhaft. "The anisotropy of the small scale structure in high Reynolds number $(\mathrm{R}(\lambda) \sim 1000)$ turbulent shear flow". In: Phys. Fluids 12.11 (2000), pp. 2976-2989. DOI: 10.1063/1.1313552.

[4] H. S. Kang, S. Chester, and C. Meneveau. "Decaying turbulence in an active-grid-generated flow and comparisons with large-eddy simulation". In: J. Fluid Mech. 480.48o (2003), pp. 129-16o. Dor: 10.1017/ S0022112002003579.

[5] P. Sagaut and C. Cambon. Homogeneous turbulence dynamics. 2018. DoI: 10. 1007/978-3-319-73162-9.

[6] K. P. Griffin et al. "Control of long-range correlations in turbulence". In: Exp. Fluids 60.4 (Apr. 2019), p. 55. DOI: 10.1007/s00348-019-26981.

[7] N. Hutchins et al. "A direct measure of the frequency response of hotwire anemometers: temporal resolution issues in wall-bounded turbulence". In: Exp. Fluids 56.1 (2015). DOI: 10.1007/s00348-014-1856-8.

[8] H. Le-The et al. "Fabrication of free-standing Pt nanowires used as thermal anemometry probes for turbulence measurements". In: Nat. Microsystems Nanoeng. under consideration (2020).

[9] D. Buaria. "Lagrangian Investigations of Turbulent Dispersion and Mixing Using Petascale Computing". PhD thesis. Georgia Institute of Technology, 2016.

[10] N. Mordant et al. "Measurement of lagrangian velocity in fully developed turbulence". In: Phys. Rev. Lett. (2001). DoI: 10.1103/PhysRevLett. 87.214501.

[11] N. Mordant, E. Lévêque, and J.-F. Pinton. “Experimental and numerical study of the Lagrangian dynamics of high Reynolds turbulence". In: New J. Phys. 6 (Sept. 2004), pp. 116-116. DOI: 10.1088/1367-2630/6/1/ 116. 
[12] S. R. Hanna. "Lagrangian and Eulerian time-scale relations in the daytime boundary layer ( Boulder, Colorado)." In: J. Appl. Meteorol. (1981). DOI: $10.1175 / 1520-0450$ (1981) 020<0242 :LAETSR>2 . 0 . CO; 2.

[13] N. Ouellette et al. "An experimental study of turbulent relative dispersion models New Journal of Physics An experimental study of turbulent relative dispersion models". In: New J. Physics, Inst. Phys. Open Access Journals 8 (2006), p. 109. DOI: 10.1088/1367-2630/8/6/109.

[14] F. Toschi and E. Bodenschatz. "Lagrangian Properties of Particles in Turbulence". In: Annu. Rev. Fluid Mech. 41.1 (Jan. 2009), pp. 375-404. DOI: 10.1146/annurev .fluid.010908.165210.

[15] S. B. Pope. "Lagrangian PDF Methods for Turbulent Flows". In: Annu. Rev. Fluid Mech. 26.1 (Jan. 1994), pp. 23-63. DoI: 10.1146/annurev.fl. 26.010194 .000323$.

[16] M. A. Strunin and T. Hiyama. "Spectral Structure of Small-Scale Turbulent and Mesoscale Fluxes in the Atmospheric Boundary Layer over a Thermally Inhomogeneous Land Surface". In: Boundary-Layer Meteorol. 117.3 (Dec. 2005), pp. 479-510. DOI: 10.1007/s10546-005-2188-2.

[17] A. Kolmogorov. "The Local Structure of Turbulence in Incompressible Viscous Fluid for Very Large Reynolds' Numbers". In: Dokl. Akad. Nauk SSSR 30 (1941), pp. 301-305.

[18] M. Sinhuber. "On the Scales of Turbulent Motion at High Reynolds Numbers". PhD thesis. 2015.

[19] L. Mydlarski and Z. Warhaft. "On the onset of high-Reynolds-number grid-generated wind tunnel turbulence". In: J. Fluid Mech. 320.-1 (Aug. 1996), p. 331. DOI: 10.1017/S0022112096007562.

[20] L. Neuhaus, J. Peinke, and M. Hölling. "High Reynolds number turbulence generation by active grid and wind tunnel control". In: Eur. Turbul. Conf. 17. 2019.

[21] B. Rousset et al. "Superfluid high REynolds von Kármán experiment". In: Rev. Sci. Instrum. 85.10 (Oct. 2014), p. 103908. DOI: 10 . 1063/1. 4897542.

[22] S. Pietropinto et al. "Superconducting instrumentation for high Reynolds turbulence experiments with low temperature gaseous helium". In: Phys. C Supercond. 386 (Apr. 2003), pp. 512-516. DOI: 10.1016/S09214534 (02) 02115-9. 
[23] K. R. Sreenivasan. "The energy dissipation in turbulent shear flows". In: Symp. Dev. Fluid Dyn. Aerosp. Eng. Bangalore, 1995, pp. 159-190.

[24] D. A. Donzis and K. R. Sreenivasan. "The bottleneck effect and the Kolmogorov constant in isotropic turbulence". In: J. Fluid Mech. 657 (2010), pp. 171-188. DOI: 10.1017/S0022112010001400.

[25] R. A. Antonia et al. "Finite Reynolds number effect and the 4/5 law". In: Phys. Rev. Fluids 4 (2019). DOI: 10.1103/PhysRevFluids . 4.084602.

[26] R. A. Antonia et al. "Approach to the 4/5 law in homogeneous isotropic turbulence". In: J. Fluid Mech. 550.1 (2006), pp. 175-184. DoI: 10.1017/ S0022112005008438.

[27] K. P. Iyer, K. R. Sreenivasan, and P. K. Yeung. "Reynolds number scaling of velocity increments in isotropic turbulence". In: Phys. Rev. E 95.2 (2017), pp. 4-9. DOI: 10.1103/PhysRevE.95.021101.

[28] K. P. Iyer, K. R. Sreenivasan, and P. K. Yeung. "Scaling exponents saturate in three-dimensional isotropic turbulence". In: 054605 (2020), pp. 1-10. DOI: 10.1103/PhysRevFluids.5.054605.

[29] Y. Tsuji. "Intermittency effect on energy spectrum in high-Reynolds number turbulence". In: Phys. Fluids 16.5 (2004), pp. 11-15. DOI: 10 . 1063/1.1689931.

[30] T. Ishihara, T. Gotoh, and Y. Kaneda. "Study of High-Reynolds Number Isotropic Turbulence by Direct Numerical Simulation". In: Annu. Rev. Fluid Mech. 41.1 (Jan. 2009), pp. 165-180. DOI: 10.1146/annurev . fluid.010908.165203.

[31] U. Frisch. Turbulence. Cambridge University Press, Nov. 1995. DOI: 10. 1017/CB09781139170666.

[32] S. G. Saddoughi, Veeravalli, and S. V. "Local isotropy in turbulent boundary layers at high Reynolds number". In: J. Fluid Mech. 268 (1994), pp. 333-372. DOI: 10.1017/S0022112094001370.

[33] R. Benzi et al. "Extended self-similarity in turbulent flows". In: Phys. Rev. E 48.1 (1993), pp. 29-32. DOI: 10.1103/PhysRevE.48. R29.

[34] M. Sinhuber, G. P. Bewley, and E. Bodenschatz. "Dissipative Effects on Inertial-Range Statistics at High Reynolds numbers". In: Phys. Rev. Lett. 119.1962 (Sept. 2016), p. 134502. DOI: 10.1103/PhysRevLett. 119. 134502. 
[35] F. Anselmet et al. "High-order velocity structure functions in turbulent shear flows". In: J. Fluid Mech. 140 (Mar. 1984), pp. 63-89. DoI: 10 . 1017/S0022112084000513.

[36] E. A. Novikov. "Mathematical model for the intermittence of turbulent flow". In: Dokl. Akad. Nauk SSSR 168.6 (1966), pp. 1279-1282.

[37] W.-X. Zhou and D. Sornette. "Evidence of intermittent cascades from discrete hierarchical dissipation in turbulence". In: Phys. D Nonlinear Phenom. 165.1-2 (May 2002), pp. 94-125. DOI: 10.1016/S0167-2789 (02) 00390-1.

[38] B. Dubrulle. "Intermittency in fully developed turbulence: Log-poisson statistics and generalized scale covariance". In: Phys. Rev. Lett. (1994). DOI: 10.1103/PhysRevLett.73.959.

[39] Z.-S. She and E. Leveque. "Universal scaling laws in fully developed turbulence". In: Phys. Rev. Lett. 72.3 (1994), pp. 336-339. DoI: 10.1103/ PhysRevLett.72.336.

[40] Z. S. She and E. C. Waymire. "Quantized energy cascade and logpoisson statistics in fully developed turbulence". In: Phys. Rev. Lett. (1995). DOI: 10.1103/PhysRevLett.74.262.

[41] H. K. Moffatt. "Singularities in fluid mechanics". In: Phys. Rev. Fluids (2019). DOI: 10.1103/PhysRevFluids.4.110502.

[42] Clay Mathematics Institute. Millenium Problems. 2016.

[43] J. C. Vassilicos. "Dissipation in Turbulent Flows". In: Annu. Rev. Fluid Mech. 47.1 (Jan. 2015), pp. 95-114. DoI: 10 . $1146 /$ annurev-fluid010814-014637.

[44] S. Risius et al. "Schneefernerhaus as a mountain research station for clouds and turbulence". In: Atmos. Meas. Tech. 8.8 (Aug. 2015), pp. 32093218. DOI: $10.5194 / \mathrm{amt}-8-3209-2015$.

[45] M. K. Verma and D. Donzis. "Energy transfer and bottleneck effect in turbulence". In: J. Phys. A Math. Theor. 40.16 (2007), pp. 4401-4412. Dor: 10.1088/1751-8113/40/16/010.

[46] P. Yang, A. Pumir, and H. Xu. "On the spectra of decaying turbulent flows". en. In: (2017), pp. 3-7.

[47] T. Leung, N. Swaminathan, and P. A. Davidson. "Geometry and interaction of structures in homogeneous isotropic turbulence". In: J. Fluid Mech. 710 (2019), p. 453. DOI: 10.1017/jfm. 2012.373. 
[48] P.-F. Yang, A. Pumir, and H. Xu. "Generalized self-similar spectrum and the effect of large-scale in decaying homogeneous isotropic turbulence". In: New J. Phys. 20.10 (Oct. 2018), p. 103035. DOI: 10.1088/13672630/aae72d.

[49] K. P. Iyer et al. "Oscillations modulating power law exponents in isotropic turbulence: Where experiments and simulations agree and differ". In: (Apr. 2020), pp. 1-5.

[5o] M. K. Verma et al. "Local shell-to-shell energy transfer via nonlocal interactions in fluid turbulence". In: Pramana 65.2 (Aug. 2005), pp. 297310. DOI: $10.1007 /$ BF02898618.

[51] C. C. Lalescu and M. Wilczek. "How tracer particles sample the complexity of turbulence". In: New J. Phys. 20.1 (2018), p. 013001. DOI: 10 . 1088/1367-2630/aa8ecd.

[52] L. Bentkamp, C. C. Lalescu, and M. Wilczek. "Persistent accelerations disentangle Lagrangian turbulence". In: Nat. Commun. 10.1 (Dec. 2019), p. 3550. DOI: 10.1038/s41467-019-11060-9.

[53] B. Sawford. "T URBULENT R ELATIVE D ISPERSION". In: Annu. Rev. Fluid Mech. 33.1 (Jan. 2001), pp. 289-317. DOI: 10.1146/annurev.fluid. 33.1.289.

[54] A. S. Monin and A. M. Yaglom. Statistical Fluid Mechanics Vol. II. Dover, 1975.

[55] B. M. O. HEPPE. “Generalized Langevin equation for relative turbulent dispersion". In: J. Fluid Mech. 357 (Feb. 1998), pp. 167-198. DOI: 10 . 1017/S0022112097008069. 


\section{Acknowledgements}

This work would not have been possible without the support of the people that surrounded me during its preparation.

First and foremost I thank Prof. Eberhard Bodenschatz for giving me the opportunity to work in his group at the MPI, for envisioning and building perhaps the single most perfect turbulence experiment and trusting me with handling it. The independence he left me was motivating and a challenge I learned to grow with. I also thank him for the patience, trust, and inspiration when things went less smooth than anticipated.

I thank Prof. Greg Bewley for his constant support, sharing his scientific wisdom and experience, being critical when I was too enthusiastic and finding excitement when I did not. This thesis would not exist without your patient support and encouragement.

Dr. Michael Wilczek provided theoretical background and a perspective from someone with great knowledge about the topic, but less emotional attachment to our work. Your friendly, calm, and systematic way to organise communications of and about science served as an ideal. Thank you!

I thank Prof. Niemeyer for providing important consultations and perspectives from outside the field of turbulence as member of my Thesis Advisory Committee. I thank Prof. Dillmann, and Prof. Tilgner for serving on my Thesis commission and discussing this work during my defense.

Dr. Michael Sinhuber left behind a working experimental setup and highquality experimental data it was a pleasure to build upon. He shared the tunnel's secrets and gave most helpful replies to any question that I had. Thank you!

I thank Prof. Markus Hultmark and Yuyang Fan for selflessly sending us NSTAPs, and patiently explaining their usage.

Greg Bewley envisioned the realisation of the active grid, Kevin Griffin and Nathaniel Wei wrote a great code to operate it. Without this work, this thesis would not have been possible.

Dr. Jan Molacek found a way to quickly provide me with tracks from the 
crude initial videos that we took from the LPT setup. Thank you!

Dr. Freja Nordsiek for giving me a head-start at particle tracking by finding the perfect particles, and providing solutions to many small problems before I even knew of their existence

I would like to thank Prof. Alain Pumir for being incredibly approachable, being open about my ideas and sharing his wisdom.

I would further like to thank Dr. John Lawson for friendly advices, proofreading parts of the thesis, and a shared fascination for fundamental turbulence experiments.

The group's technicians Artur Kubitzek, Andreas Renner, and Andreas Kopp were indispensable support, shared their experience about the tunnel and their overview about anything technical. Thank you also for creating a relaxed environment in the experimental hall and committing even to the most crazy ideas of mine.

The machine shop under the guidance of Udo Schminke is a highlight of the institute. Thank you for helpful discussions, your patience with my incomplete designs and for all those jobs very well done, in particular for the active grid and the camera sled!

I would like to thank the electric workshop, who designed anemometry circuits after my rough sketches. Thank you also for your patience with the crude knowledge of electronics that my physics education provided me with.

Thank you, Tefa for most helpful discussions and math help. Thanks, Guus Bertens for patiently discussing problems about my particle tracking setup, sharing your experiences with the Trumpf laser and the Phantom cameras, and creating a system that allows the convenient acquisition of particle tracking data. Antonio Ibañez was of great help in finalising the particle tracking, for which I am equally thankful. Marcel Schröder proofread parts of the thesis.

Besides the professional support, this work would not have been possible without the friendships inside and outside the institute that provided everything from emotional support, lessons for life, new energy and inspiration or simply a welcome distraction. Among these are Arne, Guus, Silke and her track and field group, Larissa, Daniela, Canan, Merlin, Bruce Springsteen, Brandi Carlile, and Walter.

Tefa, thank you for being my very best friend every single day through thick and thin.

Finally, I would like to thank my beloved parents whose support, wisdom, inspiration, financial aid, and encouragement brought me where I am. 
Appendices 



\section{Appendix A: Parts of the particle tracking system}

The following table lists the basic parts used for assembly of the particle tracking system in detail. Its design and properties are described in Ch. 10. 


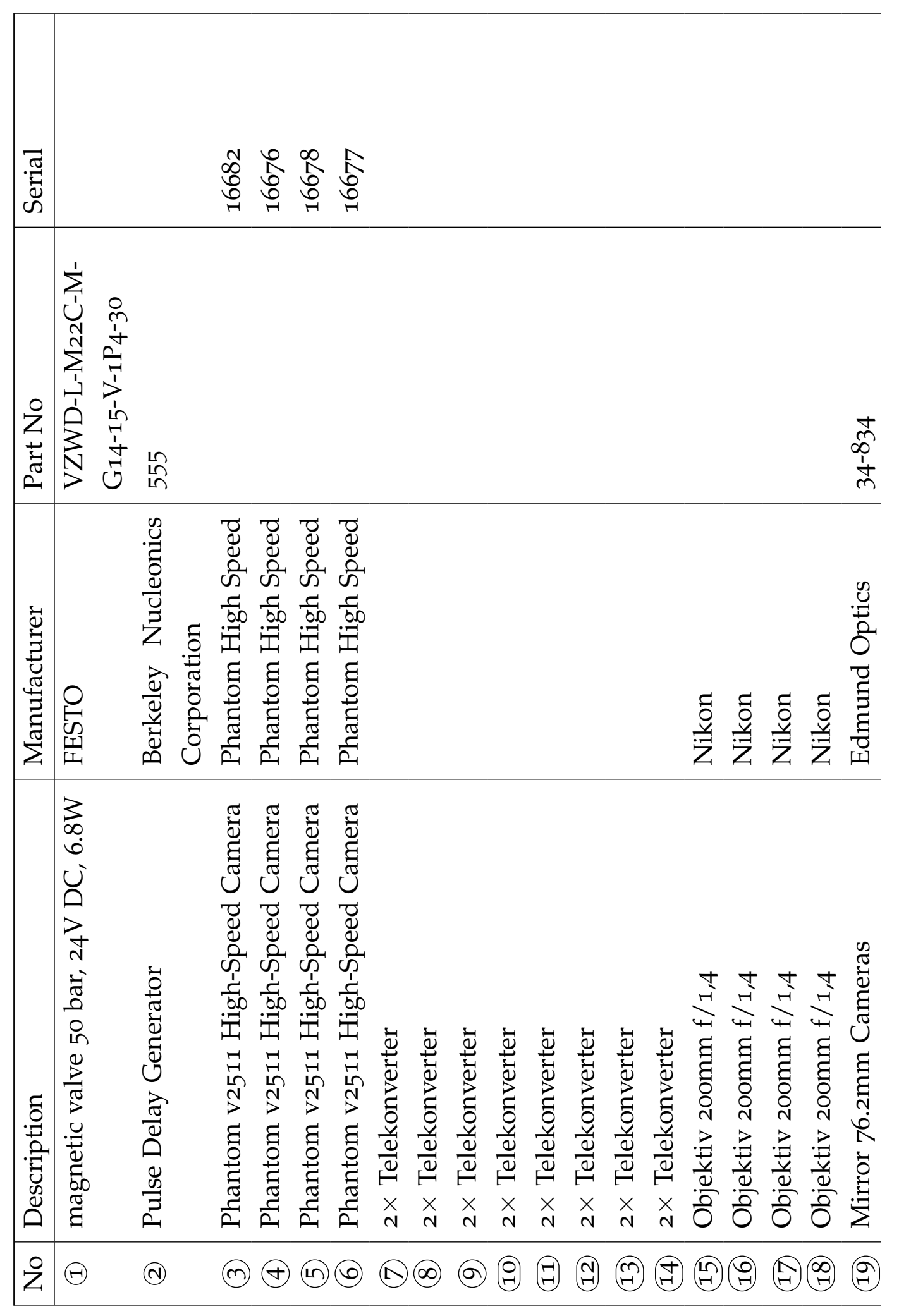




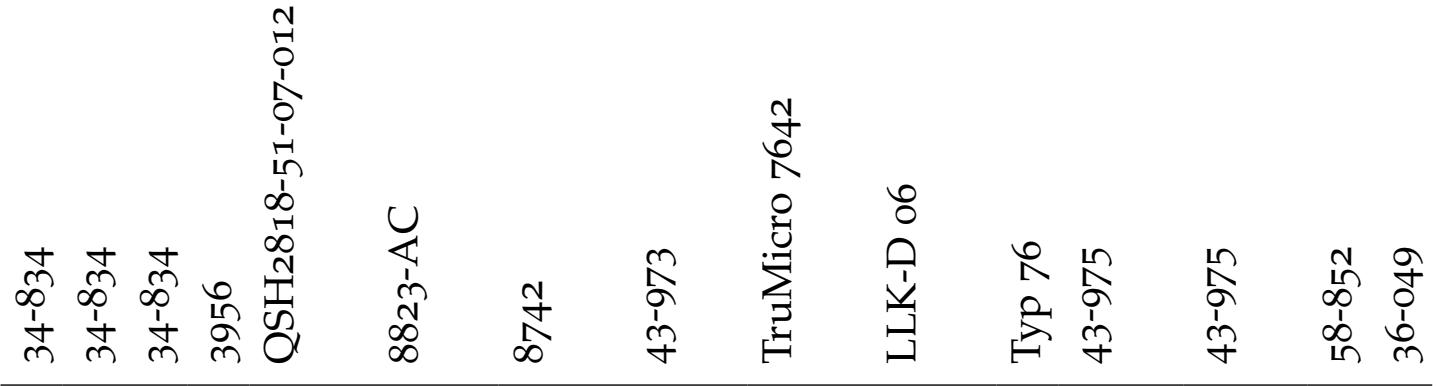

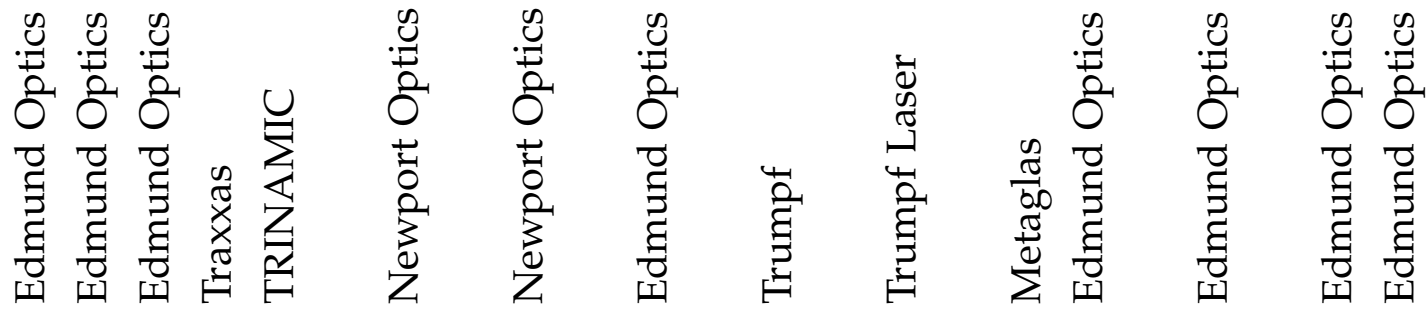

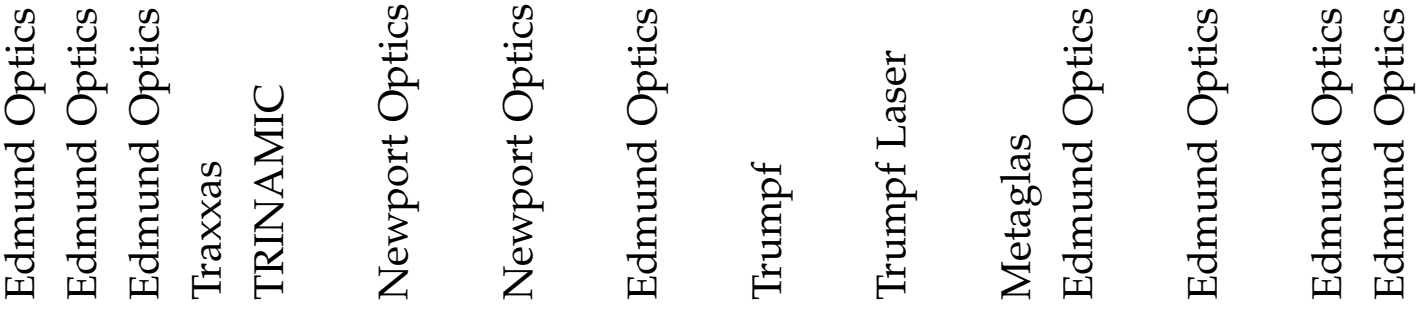

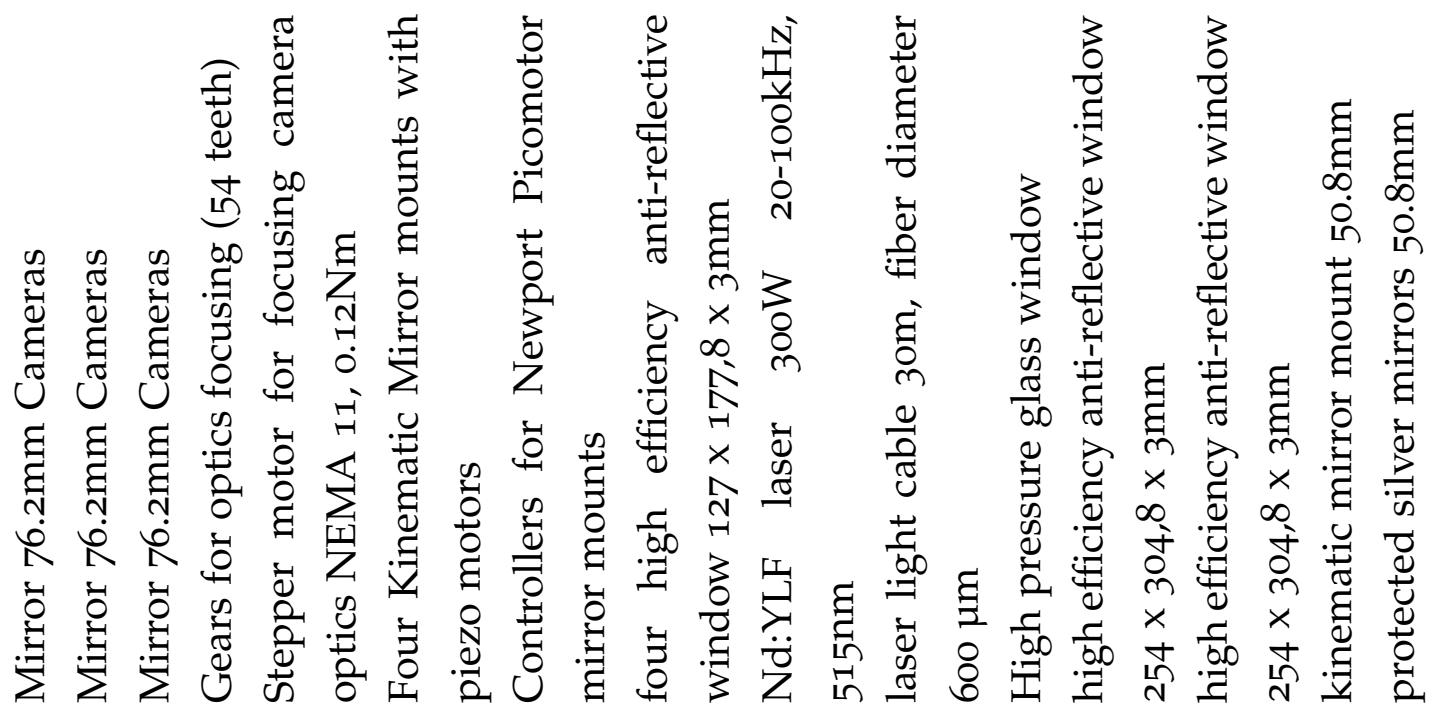
() (ㄱ) (ָ) (ิ)
(ब) (2)
(
(ู)
() (
(2)
(m) () 


\section{Appendix B: Hot Wire Datasets}

The following table provides an overview over the datasets that were the basis for 4 and Pt. II. Dissipation rate $\varepsilon$, and therefore $\eta, \lambda$, and $R_{\lambda}$ were estimated from the gradients of the one-dimensional velocity field, errors are estimated smaller than $\pm 8 \%$, based on the results of Sec. 5.3.3. L was estimated as $\int_{0}^{r_{0}} C_{11}(r) d r$, where $r_{0}$ is the smallest separation, where the correlation function $C_{11}$ crosses 0 . The errors of this quantity are estimated as $4 \%$. The errors for fluctuation velocity $u_{R M S}$ and mean flow velocity $U$ are predominantly due to calibration, i.e. $\approx 4 \%$. The facility pressure could be measured up to \pm 0.01 bar. $v$ was taken from Hoogland et al. ${ }^{1}$ interfaced by an in-house program (courtesy of Prof. G. Ahlers).

The probe positions are measured as follows: $x$ : Probe position away from the active grid. $y$ : Distance from tunnel centerline. $z$ : height from tunnel floor.

Details on the grid protocols can be found in Sec. 4.2.

${ }^{1}$ J. H. Hoogland, H. R. Van Den Berg, and N. J. Trappeniers. "Measurements of the viscosity of sulfur hexaflouride up to 100 bar by a capillary-flow viscometer". In: Phys. A Stat. Mech. its Appl. (1985). DOI: 10.1016/0378-4371(85)90160-8 


\begin{tabular}{|c|c|c|c|c|c|c|c|c|c|c|}
\hline 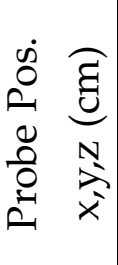 & 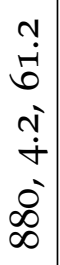 & 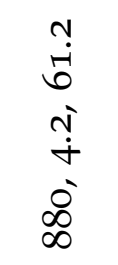 & 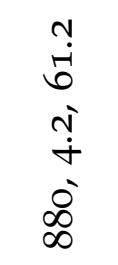 & 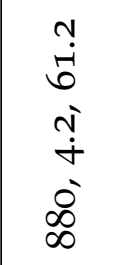 & 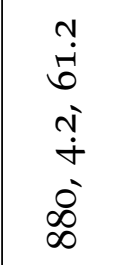 & 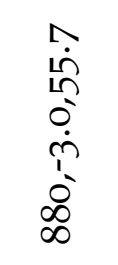 & 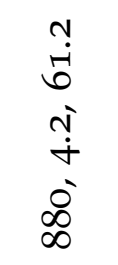 & $\begin{array}{c}0 \\
\dot{0} \\
\dot{0} \\
0 \\
\dot{+} \\
0 \\
\infty \\
\infty\end{array}$ & $\begin{array}{l}\Upsilon \\
\dot{n} \\
10 \\
0 \\
\dot{n} \\
1 \\
0 \\
\infty \\
\infty\end{array}$ & $\begin{array}{l}\nwarrow \\
\dot{1} \\
\underline{1} \\
0 \\
\dot{n} \\
1 \\
0 \\
\infty \\
\infty\end{array}$ \\
\hline 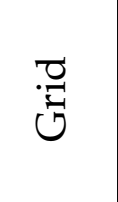 & $\begin{array}{l}\tilde{Q} \\
0\end{array}$ & 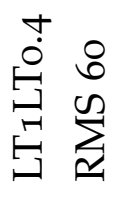 & 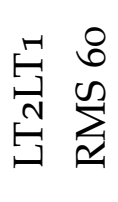 & $\begin{array}{ll}\stackrel{m}{\stackrel{m}{H}} & 8 \\
\stackrel{m}{m} & \sum \\
\stackrel{n}{\omega} & \infty\end{array}$ & 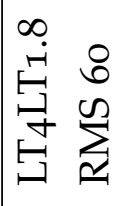 & 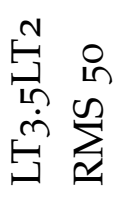 & 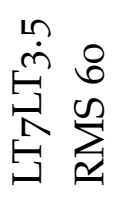 & 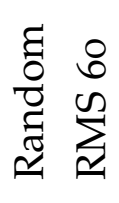 & 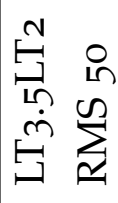 & 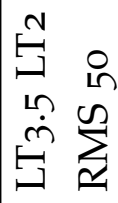 \\
\hline 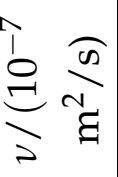 & 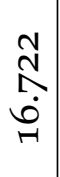 & $\stackrel{N}{\underline{H}}$ & $\stackrel{\vartheta}{\hat{\sigma}}$ & $\stackrel{\sim}{\sim}$ & $\stackrel{\sim}{\sim}$ & 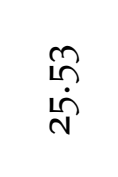 & 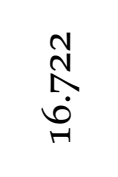 & $\begin{array}{l}\text { Ln } \\
\infty \\
\text { i }\end{array}$ & $\begin{array}{l}\stackrel{+}{\leftarrow} \\
\stackrel{\leftrightarrow}{H}\end{array}$ & $\begin{array}{l}\infty \\
\stackrel{n}{\Lambda}\end{array}$ \\
\hline$\sum_{\text {है }}^{\text {a }}$ & $\stackrel{\infty}{\stackrel{\infty}{+}}$ & $\stackrel{\infty}{\stackrel{\infty}{+}}$ & 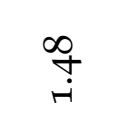 & $\stackrel{\stackrel{+}{+}}{r}$ & $\underset{+}{\stackrel{+}{+}}$ & $\begin{array}{l}1 \\
\infty \\
0 \\
0\end{array}$ & $\stackrel{\infty}{\stackrel{\infty}{+}}$ & $\stackrel{\sigma}{\grave{\Lambda}}$ & $\begin{array}{l}\stackrel{\infty}{\llcorner} \\
\stackrel{\leftrightarrow}{n}\end{array}$ & $\begin{array}{c}\infty \\
\stackrel{\infty}{1} \\
\dot{r}\end{array}$ \\
\hline$\frac{\sqrt[\infty]{\Xi}}{\Xi}$ & 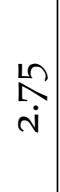 & $\underset{\text { Tे }}{\text { ก }}$ & $\frac{\text { Tे }}{\text { i⿱ }}$ & $\begin{array}{l}m \\
\dot{i}\end{array}$ & $\begin{array}{l}\stackrel{L}{b} \\
\stackrel{i}{i}\end{array}$ & $\stackrel{+\infty}{\infty}$ & $\stackrel{\sim}{\stackrel{N}{i}}$ & $\stackrel{\vec{r}}{\hat{i}}$ & $\stackrel{\stackrel{\leftrightarrow}{\leftrightarrow}}{\dot{m}}$ & $\begin{array}{l}\stackrel{\theta}{\leftrightarrow} \\
\dot{m}\end{array}$ \\
\hline 章 & $\begin{array}{l}8 \\
\stackrel{N}{N}\end{array}$ & $\begin{array}{l}8 \\
\text { in } \\
m\end{array}$ & $\begin{array}{l}8 \\
\stackrel{1}{m} \\
m\end{array}$ & $\begin{array}{l}8 \\
\text { nn } \\
m\end{array}$ & $\begin{array}{l}8 \\
\text { in } \\
m\end{array}$ & \begin{tabular}{l}
8 \\
8 \\
8 \\
\hdashline
\end{tabular} & $\begin{array}{l}8 \\
\stackrel{\circ}{m} \\
m\end{array}$ & $\begin{array}{l}8 \\
8 \\
\stackrel{7}{+}\end{array}$ & 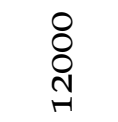 & $\begin{array}{l}8 \\
8 \\
8 \\
-1\end{array}$ \\
\hline $\begin{array}{l}0 \\
0 \\
0 \\
0 \\
0\end{array}$ & $\stackrel{m}{\sim}$ & $\stackrel{M}{\sim}$ & $\stackrel{M}{\sim}$ & $\stackrel{m}{\rightarrow}$ & $\stackrel{M}{\rightarrow}$ & $\underset{\sim}{\infty}$ & $\stackrel{M}{\sim}$ & $\stackrel{n}{N}$ & $\stackrel{\infty}{\infty}$ & $\stackrel{\infty}{\infty}$ \\
\hline 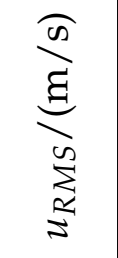 & $\begin{array}{l}+ \\
0 \\
\dot{0}\end{array}$ & 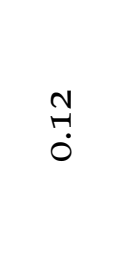 & $\begin{array}{l}\stackrel{6}{-} \\
\stackrel{-}{0}\end{array}$ & $\stackrel{\sim}{\sim}$ & $\begin{array}{c}\infty \\
\stackrel{+}{0} \\
0\end{array}$ & $\begin{array}{c}\infty \\
\text { ֻ } \\
\dot{0}\end{array}$ & 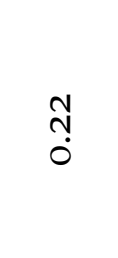 & $\stackrel{7}{7}$ & $\begin{array}{l}\dot{m} \\
\dot{0}\end{array}$ & 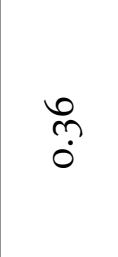 \\
\hline 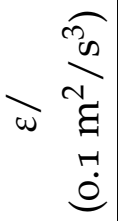 & 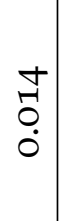 & $\begin{array}{l}\text { ָे } \\
\stackrel{+}{+} \\
0\end{array}$ & $\begin{array}{l}1 \\
\text { กิ } \\
0\end{array}$ & $\begin{array}{l}\text { ठ } \\
\text { Tุ } \\
0\end{array}$ & $\begin{array}{l}\text { Nָ } \\
\text { ִָ } \\
0\end{array}$ & $\begin{array}{l}\infty \\
\infty \\
i n \\
i\end{array}$ & $\begin{array}{l}\text { จे } \\
\text { ஸे }\end{array}$ & $\begin{array}{l}-1 \\
0 \\
0 \\
0\end{array}$ & 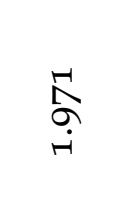 & 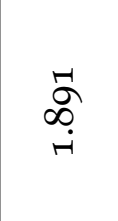 \\
\hline$\Xi$ & $\begin{array}{l}0 \\
? \\
0\end{array}$ & $\stackrel{\overbrace{}}{\stackrel{0}{0}}$ & 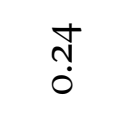 & $\begin{array}{l}\text { Nִ } \\
0\end{array}$ & กิ & 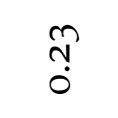 & $\stackrel{+}{\dot{0}}$ & $\hat{\overbrace{}}$ & $\begin{array}{l}\stackrel{L}{N} \\
\stackrel{0}{0}\end{array}$ & 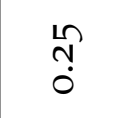 \\
\hline 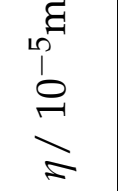 & $\begin{array}{l}\hat{\tilde{n}} \\
\text { ते } \\
\text { ते }\end{array}$ & $\begin{array}{l}\qquad \\
\infty \\
\infty \\
\dot{\sim} \\
\sim\end{array}$ & $\begin{array}{l}\underset{v}{N} \\
\underset{n}{N} \\
\vec{H}\end{array}$ & \begin{tabular}{l}
$\infty$ \\
\multirow{1}{L}{} \\
$\stackrel{i}{H}$ \\
$\vec{H}$
\end{tabular} & 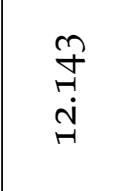 & $\begin{array}{l}\text { ते } \\
0 \\
\sigma\end{array}$ & 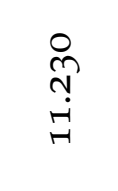 & $\begin{array}{l}\underline{\omega} \\
\hat{\alpha} \\
\dot{n}\end{array}$ & $\begin{array}{l}\text { ठ্. } \\
\qquad \\
0\end{array}$ & 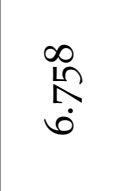 \\
\hline$\widetilde{\alpha}$ & $\underset{F}{\stackrel{J}{*}}$ & $\stackrel{m}{\underset{\gamma}{\forall}}$ & $\stackrel{m}{\stackrel{n}{*}}$ & స్ & ర్రా & 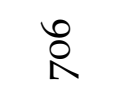 & ๖ & $\begin{array}{l}\infty \\
\infty \\
\infty\end{array}$ & $\stackrel{m}{a}$ & $\ddot{n}$ \\
\hline
\end{tabular}




\begin{tabular}{|c|c|c|c|c|c|c|c|c|c|c|}
\hline 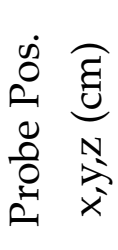 & $\begin{array}{c}0 \\
\dot{0} \\
\dot{b} \\
0 \\
\dot{0} \\
0 \\
\infty \\
\infty\end{array}$ & $\begin{array}{l}0 \\
\dot{+} \\
\dot{0} \\
0 \\
\dot{+} \\
0 \\
0 \\
\infty\end{array}$ & $\begin{array}{l}\hat{N} \\
\hat{n} \\
\tilde{0} \\
\dot{1} \\
1 \\
0 \\
\infty \\
\infty\end{array}$ & $\begin{array}{l}0 \\
\dot{1} \\
6 \\
0 \\
\dot{+} \\
0 \\
0 \\
\infty \\
\infty\end{array}$ & $\begin{array}{c}\hat{N} \\
\hat{n} \\
\hat{0} \\
\dot{n} \\
1 \\
0 \\
\infty \\
\infty\end{array}$ & $\begin{array}{l}0 \\
\dot{-1} \\
\dot{0} \\
0 \\
\dot{+} \\
0 \\
\infty \\
\infty \\
\infty\end{array}$ & $\begin{array}{c}0 \\
\dot{1} \\
0 \\
0 \\
\dot{0} \\
\dot{0} \\
\infty \\
\infty\end{array}$ & 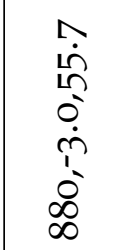 & $\begin{array}{l}\Lambda \\
\dot{1} \\
\hat{0} \\
\dot{1} \\
1 \\
1 \\
8 \\
\infty\end{array}$ & $\begin{array}{l}0 \\
\dot{1} \\
0 \\
0 \\
\dot{+} \\
0 \\
\infty \\
\infty\end{array}$ \\
\hline 总 & 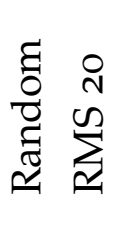 & 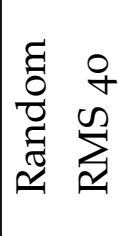 & 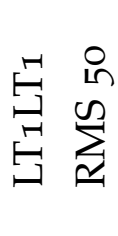 & 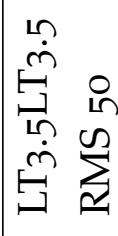 & 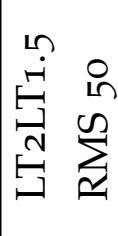 & 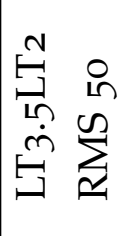 & 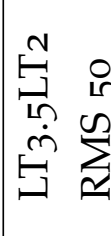 & 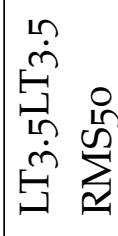 & 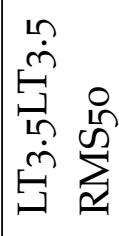 & 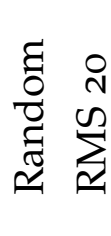 \\
\hline 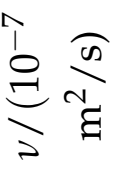 & $\begin{array}{l}n \\
\text { no } \\
\text { i }\end{array}$ & $\begin{array}{l}\text { Ln } \\
\infty \\
\text { i }\end{array}$ & $\begin{array}{l}0 \\
\stackrel{1}{0} \\
\dot{+}\end{array}$ & $\begin{array}{l}\text { तु } \\
\infty \\
\infty\end{array}$ & $\begin{array}{l}0 \\
\stackrel{-}{0} \\
\dot{+}\end{array}$ & $\stackrel{\stackrel{M}{\hookrightarrow}}{\stackrel{m}{n}}$ & 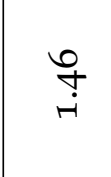 & $\begin{array}{l}\hat{\sigma} \\
\dot{n}\end{array}$ & $\begin{array}{l}\hat{\alpha} \\
\dot{m}\end{array}$ & 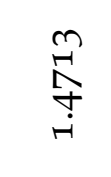 \\
\hline 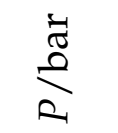 & 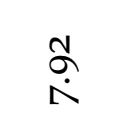 & 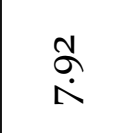 & $\begin{array}{l}\hat{n} \\
\dot{\hat{n}}\end{array}$ & $\begin{array}{l}\stackrel{L}{\infty} \\
i\end{array}$ & $\begin{array}{l}\text { ڤn } \\
\dot{n}\end{array}$ & $\begin{array}{l}\stackrel{1}{\infty} \\
\stackrel{\infty}{+}\end{array}$ & $\stackrel{ }{\stackrel{-}{+}}$ & $\begin{array}{l}\text { ஸू } \\
\dot{n}\end{array}$ & $\begin{array}{l}\stackrel{L}{\alpha} \\
\dot{n}\end{array}$ & $\begin{array}{l}\text { ț } \\
\dot{J}\end{array}$ \\
\hline$\frac{\sqrt[\infty]{\Xi}}{\Xi}$ & $\begin{array}{l}\hat{\alpha} \\
\dot{i}\end{array}$ & $\begin{array}{c}\infty \\
\infty \\
i \\
i\end{array}$ & 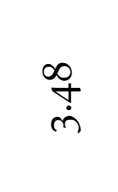 & $\begin{array}{l}\dot{0} \\
\dot{m}\end{array}$ & $\begin{array}{l}\dot{1} \\
\dot{n} \\
\dot{m}\end{array}$ & 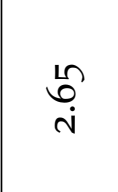 & $\begin{array}{l}\infty \\
\infty \\
i \\
i\end{array}$ & $\begin{array}{l}\mathscr{\ell} \\
\dot{m}\end{array}$ & $\begin{array}{l}\hat{\sigma} \\
\dot{m}\end{array}$ & $\begin{array}{l}\mathscr{2} \\
\dot{m}\end{array}$ \\
\hline 盖焉 & $\begin{array}{l}7 \\
8 \\
8 \\
8\end{array}$ & $\begin{array}{l}7 \\
8 \\
8 \\
8\end{array}$ & \begin{tabular}{l}
8 \\
8 \\
$\circ$ \\
\hdashline
\end{tabular} & $\begin{array}{l}8 \\
8 \\
8 \\
8\end{array}$ & 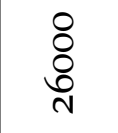 & $\begin{array}{l}8 \\
8 \\
8\end{array}$ & $\begin{array}{l}8 \\
\delta \\
\infty \\
\infty\end{array}$ & $\begin{array}{l}8 \\
8 \\
8 \\
8\end{array}$ & $\begin{array}{l}8 \\
8 \\
10 \\
m\end{array}$ & $\begin{array}{l}8 \\
8 \\
\stackrel{1}{1} \\
m\end{array}$ \\
\hline $\begin{array}{l}0 \\
0 \\
0 \\
01\end{array}$ & $\stackrel{\mathrm{n}}{\mathrm{N}}$ & $\stackrel{n}{\sim}$ & הิ & $\stackrel{\operatorname{Ln}}{\mathrm{N}}$ & $\widehat{\widehat{N}}$ & $\stackrel{\stackrel{n}{N}}{n}$ & $\stackrel{\operatorname{n}}{\sim}$ & ה & तิ & $\stackrel{\operatorname{Ln}}{\mathrm{N}}$ \\
\hline$\underbrace{\stackrel{\infty}{\Xi}}_{\substack{\Xi \\
\Xi}}$ & $\stackrel{m}{\stackrel{m}{\circ}}$ & $\stackrel{-}{\sim}$ & 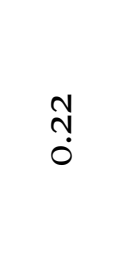 & $\stackrel{+}{\grave{0}}$ & 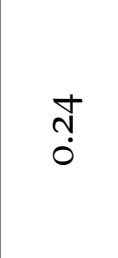 & 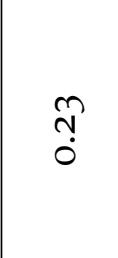 & 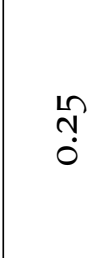 & $\ddot{n}$ & $\stackrel{m}{\dddot{0}}$ & 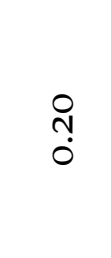 \\
\hline 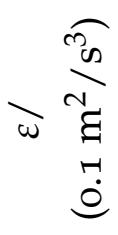 & 每 & 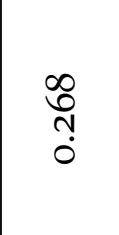 & $\stackrel{\infty}{+}$ & ت & 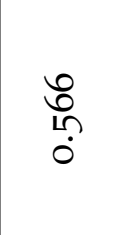 & 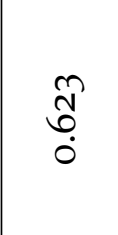 & ָָ & $\begin{array}{l}n \\
\infty \\
\infty \\
0\end{array}$ & $\begin{array}{c}0 \\
\infty \\
0 \\
\\
ن\end{array}$ & $\begin{array}{l}\infty \\
10 \\
n \\
0\end{array}$ \\
\hline$\Xi$ & $\stackrel{m}{?}$ & $\stackrel{n}{\sim}$ & a & $\ddot{m}$ & $\stackrel{m}{\grave{0}}$ & $\stackrel{\text { ָ̦ }}{0}$ & సָ & $\stackrel{1}{\dddot{0}}$ & $\stackrel{+}{\tilde{0}}$ & $\stackrel{n}{?}$ \\
\hline 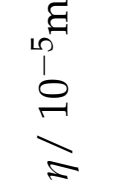 & $\begin{array}{l}\hat{N} \\
\dot{n}\end{array}$ & $\begin{array}{l}\stackrel{L}{\infty} \\
0 \\
\dot{n}\end{array}$ & $\begin{array}{l}\stackrel{8}{+} \\
\dot{n} \\
\dot{n}\end{array}$ & $\begin{array}{l}\mathscr{0} \\
\infty \\
\dot{+}\end{array}$ & $\begin{array}{l}\hat{\widehat{N}} \\
\dot{n}\end{array}$ & 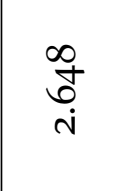 & ָु & $\begin{array}{l}\hat{\alpha} \\
\text { à } \\
\text { in }\end{array}$ & 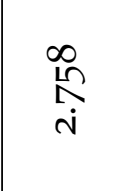 & $\begin{array}{l}\widehat{N} \\
\stackrel{\sim}{N}\end{array}$ \\
\hline$\widetilde{z}$ & $\underset{\sim}{\stackrel{0}{O}}$ & 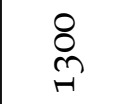 & $\begin{array}{l}\stackrel{n}{O} \\
\stackrel{M}{r}\end{array}$ & 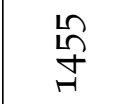 & $\stackrel{-\infty}{+}$ & $\begin{array}{l}\stackrel{L}{n} \\
\stackrel{n}{\sim}\end{array}$ & $\stackrel{\widehat{N}}{\widehat{N}}$ & $\begin{array}{c}\text { ñ } \\
\stackrel{N}{2}\end{array}$ & 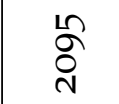 & 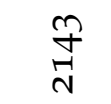 \\
\hline
\end{tabular}




\begin{tabular}{|c|c|c|c|c|c|c|c|c|c|c|}
\hline 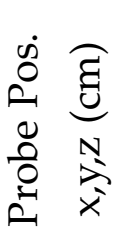 & $\begin{array}{l}\Upsilon \\
\dot{n} \\
0 \\
0 \\
\dot{1} \\
1 \\
0 \\
\infty\end{array}$ & 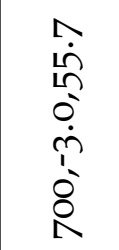 & $\begin{array}{l}0 \\
\dot{0} \\
6 \\
0 \\
\dot{0} \\
\dot{0} \\
0 \\
\infty\end{array}$ & $\begin{array}{l}\hat{N} \\
\dot{1} \\
0 \\
0 \\
\dot{1} \\
1 \\
\dot{8} \\
\delta\end{array}$ & $\begin{array}{l}\hat{N} \\
1 \hat{n} \\
0 \\
0 \\
\dot{1} \\
\vdots \\
0 \\
\infty\end{array}$ & $\begin{array}{l}\Upsilon \\
\dot{1} \\
\underline{0} \\
0 \\
\dot{1} \\
1 \\
\dot{0} \\
\infty\end{array}$ & 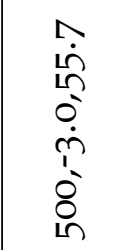 & 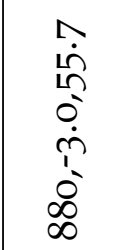 & $\begin{array}{l}\hat{1} \\
\underline{n} \\
0 \\
\dot{n} \\
\vdots \\
\dot{0}\end{array}$ & $\begin{array}{l}1 \\
1 \\
10 \\
0 \\
\dot{n} \\
\vdots \\
\dot{m} \\
\dot{+}\end{array}$ \\
\hline 苟 & $\begin{array}{l}m \\
H^{m} \\
\underbrace{+}\end{array}$ & 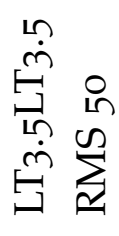 & 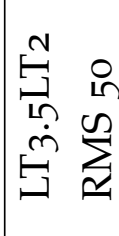 & $\begin{array}{l}\stackrel{n}{m} \circ \\
\stackrel{n}{\omega} \circ \\
\dot{m} \\
\dot{\omega}\end{array}$ & 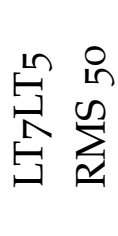 & 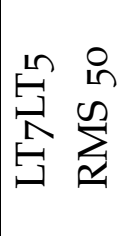 & $\begin{array}{ll}\stackrel{n}{n} & \\
\dot{m} & 0 \\
\vec{\omega} & i \\
\dot{n} & \infty \\
\dot{m} & \sum\end{array}$ & 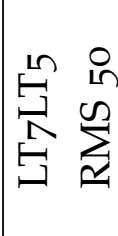 & 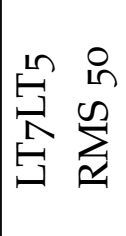 & $\begin{array}{l}\dot{m} \\
\dot{m}\end{array}$ \\
\hline 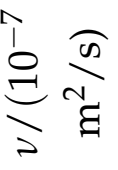 & $\begin{array}{l}\hat{\sigma} \\
\dot{m}\end{array}$ & $\begin{array}{l}\hat{\sigma} \\
\dot{m}\end{array}$ & 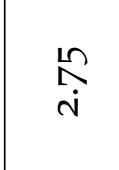 & $\begin{array}{l}\hat{\sigma} \\
\dot{n}\end{array}$ & $\begin{array}{l}\infty \\
\alpha \\
\dot{m}\end{array}$ & $\begin{array}{l}\infty \\
\alpha \\
\dot{m}\end{array}$ & $\begin{array}{l}\hat{\sigma} \\
\dot{m}\end{array}$ & $\begin{array}{l}\hat{\cong} \\
\dot{m}\end{array}$ & $\begin{array}{l}\infty \\
\alpha \\
\dot{m}\end{array}$ & $\begin{array}{l}\hat{\sigma} \\
\dot{n}\end{array}$ \\
\hline$\sum_{0}^{\text {పే }}$ & $\begin{array}{l}\widehat{\widehat{~}} \\
\dot{n}\end{array}$ & $\begin{array}{l}\stackrel{\operatorname{r}}{\hat{n}} \\
\dot{n}\end{array}$ & $\stackrel{\curvearrowright}{\infty}$ & $\begin{array}{l}\stackrel{L}{\sigma} \\
\dot{n}\end{array}$ & 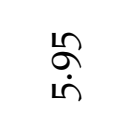 & 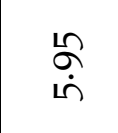 & $\begin{array}{l}\stackrel{L}{\alpha} \\
\dot{n}\end{array}$ & $\begin{array}{l}\hat{\sigma} \\
\dot{n}\end{array}$ & $\begin{array}{l}\hat{\sigma} \\
\dot{n}\end{array}$ & $\begin{array}{l}\stackrel{2}{\sigma} \\
\dot{n}\end{array}$ \\
\hline 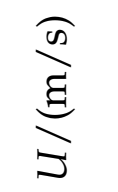 & $\begin{array}{l}\dot{J} \\
\dot{m}\end{array}$ & $\begin{array}{l}\stackrel{8}{m} \\
\dot{m}\end{array}$ & $\begin{array}{l}\mathscr{b} \\
\dot{m}\end{array}$ & $\begin{array}{l}\dot{8} \\
\dot{m}\end{array}$ & $\begin{array}{l}\stackrel{\llcorner}{\Upsilon n} \\
\dot{m}\end{array}$ & 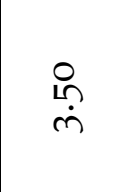 & $\begin{array}{l}\infty \\
\stackrel{\infty}{10} \\
\dot{m}\end{array}$ & $\begin{array}{l}\vec{b} \\
\dot{m}\end{array}$ & $\begin{array}{l}\text { fे } \\
\dot{m}\end{array}$ & $\begin{array}{l}\stackrel{\circ}{\Upsilon ે} \\
\dot{n}\end{array}$ \\
\hline 㝨 & 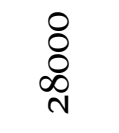 & $\begin{array}{l}8 \\
8 \\
i n \\
m\end{array}$ & $\begin{array}{l}8 \\
8 \\
8 \\
m\end{array}$ & $\begin{array}{l}8 \\
8 \\
i n \\
m\end{array}$ & $\begin{array}{l}8 \\
8 \\
i n \\
m\end{array}$ & $\begin{array}{l}8 \\
8 \\
i n \\
m\end{array}$ & $\begin{array}{l}8 \\
8 \\
i n \\
m\end{array}$ & 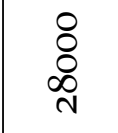 & 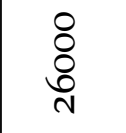 & $\begin{array}{l}8 \\
8 \\
\stackrel{1}{m}\end{array}$ \\
\hline $\begin{array}{l}0 \\
0 \\
0 \\
\text { s. }\end{array}$ & तิ & ヘิ & $\stackrel{\stackrel{n}{N}}{n}$ & הิ & तิ & तิ & तิ & तิ & तิ & तิ \\
\hline 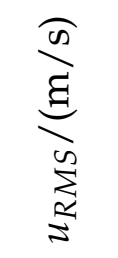 & mे & $\hat{\circ}$ & $\stackrel{m}{0}$ & $\underset{+}{+}$ & $\stackrel{\infty}{\dddot{m}}$ & $\stackrel{\circ}{+}$ & 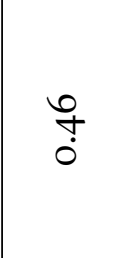 & $\stackrel{\circ}{+}$ & $\stackrel{m}{+}$ & $\begin{array}{l}\text { 무 } \\
\dot{0}\end{array}$ \\
\hline 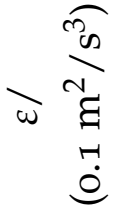 & $\begin{array}{l}\stackrel{N}{N} \\
\stackrel{+}{-} \\
\end{array}$ & $\stackrel{+}{\stackrel{m}{r}}$ & $\begin{array}{l}8 \\
8 \\
\\
\end{array}$ & $\underset{+}{\stackrel{M}{+}}$ & & $\stackrel{\vec{m}}{\stackrel{\leftrightarrow}{r}}$ & 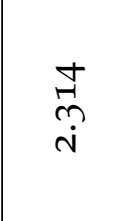 & $\begin{array}{l}\text { ำ } \\
\text { ָे } \\
\text { r }\end{array}$ & $\begin{array}{l}\stackrel{ }{2} \\
\stackrel{م}{r}\end{array}$ & 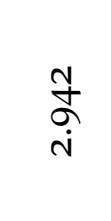 \\
\hline$\Xi$ & $\stackrel{m}{\ddot{0}}$ & $\stackrel{m}{0}$ & 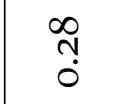 & 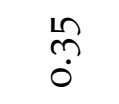 & $\stackrel{7}{+}$ & $\stackrel{\infty}{+}$ & $\begin{array}{l}\stackrel{m}{0} \\
\dot{0}\end{array}$ & $\stackrel{m}{+}$ & $\stackrel{0}{+}$ & $\stackrel{\grave{m}}{\ddot{0}}$ \\
\hline 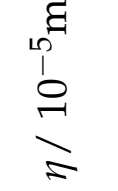 & 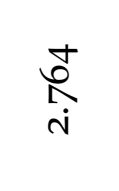 & 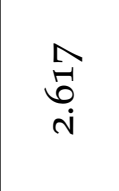 & 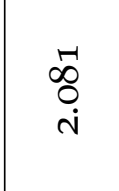 & 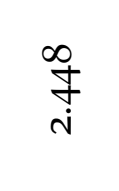 & 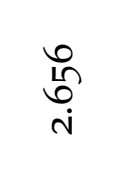 & $\begin{array}{l}\hat{N} \\
\hat{\Lambda} \\
\text { in }\end{array}$ & 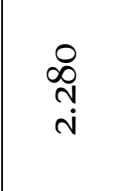 & 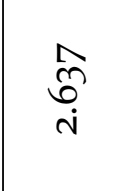 & $\begin{array}{l}\hat{\sigma} \\
\stackrel{+}{+} \\
\text { in }\end{array}$ & 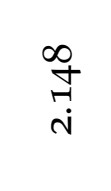 \\
\hline$\widehat{\Sigma}$ & 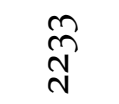 & ন্ন & 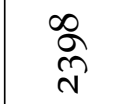 & 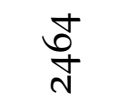 & 哭 & $\stackrel{-}{\stackrel{n}{\sigma}}$ & ठ্రి & 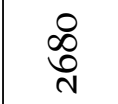 & నૂ & 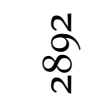 \\
\hline
\end{tabular}




\begin{tabular}{|c|c|c|c|c|c|c|}
\hline 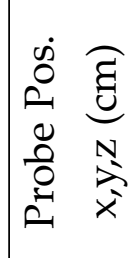 & $\begin{array}{l}1 \\
\hat{n} \\
1 \\
0 \\
\dot{1} \\
\hat{0} \\
8\end{array}$ & 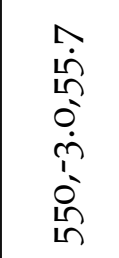 & $\begin{array}{l}r \\
\text { Nं } \\
0 \\
0 \\
\dot{1} \\
1 \\
0 \\
\infty \\
\infty\end{array}$ & $\begin{array}{l}0 \\
\dot{1} \\
\dot{0} \\
0 \\
\dot{+} \\
0 \\
0 \\
\infty \\
\infty\end{array}$ & $\begin{array}{l}0 \\
\dot{0} \\
0 \\
0 \\
\dot{+} \\
0 \\
\infty \\
\infty\end{array}$ & $\begin{array}{c}0 \\
\dot{1} \\
\dot{6} \\
0 \\
\dot{+} \\
0 \\
\infty \\
\infty\end{array}$ \\
\hline 节 & 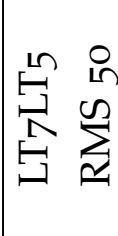 & 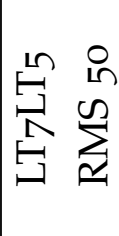 & 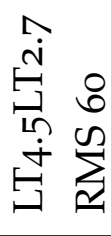 & 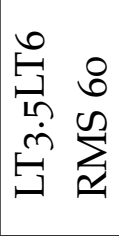 & 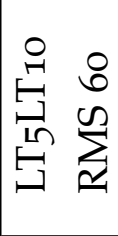 & 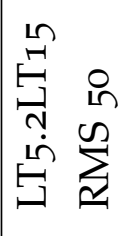 \\
\hline 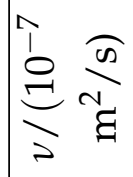 & $\begin{array}{l}\infty \\
\stackrel{\alpha}{m} \\
\dot{m}\end{array}$ & $\begin{array}{l}\infty \\
\alpha \\
\dot{m}\end{array}$ & 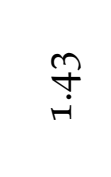 & $\underset{\stackrel{n}{+}}{\stackrel{+}{+}}$ & $\underset{+}{\stackrel{+}{+}}$ & $\underset{+}{\stackrel{\sim}{+}}$ \\
\hline$\sum_{a}^{\dot{\Xi}}$ & 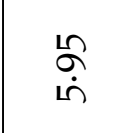 & $\begin{array}{l}\stackrel{L}{\alpha} \\
\dot{n}\end{array}$ & $\begin{array}{l}\text { ֶै } \\
\text { +̇ }\end{array}$ & $\begin{array}{l}\text { Vै } \\
\dot{H}\end{array}$ & $\begin{array}{l}\stackrel{L}{6} \\
\dot{+}\end{array}$ & $\begin{array}{l}\text { రి } \\
\dot{+}\end{array}$ \\
\hline$\frac{\sqrt{n}}{\Xi}$ & $\underset{\dot{m}}{\forall}$ & $\underset{\dot{m}}{\forall}$ & $\begin{array}{l}8 \\
\dot{m}\end{array}$ & 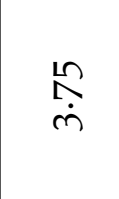 & $\begin{array}{l}\hat{\sigma} \\
\dot{m}\end{array}$ & $\begin{array}{l}\text { ô } \\
\dot{+}\end{array}$ \\
\hline 暚疍 & $\begin{array}{l}8 \\
8 \\
1 \\
m\end{array}$ & $\begin{array}{l}8 \\
8 \\
10 \\
m\end{array}$ & $\begin{array}{l}8 \\
8 \\
\text { in } \\
\text { n. }\end{array}$ & $\begin{array}{l}8 \\
\stackrel{1}{1} \\
\text { ลิ }\end{array}$ & $\begin{array}{l}8 \\
8 \\
8 \\
0\end{array}$ & 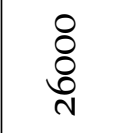 \\
\hline $\begin{array}{l}0 \\
0 \\
0 \\
0 \\
0\end{array}$ & $\hat{\sim}$ & तิ & $\stackrel{\rightarrow}{\rightarrow}$ & $\stackrel{\llcorner}{\sim}$ & $\stackrel{\llcorner}{\sim}$ & $\stackrel{\mathfrak{N}}{\mathrm{N}}$ \\
\hline$\underbrace{\stackrel{\infty}{\Xi}}_{\substack{\infty \\
\Xi}}$ & $\begin{array}{l}0 \\
+ \\
0\end{array}$ & 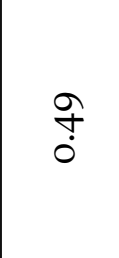 & $\begin{array}{l}\text { ָ̦ } \\
\text { Oे }\end{array}$ & $\underset{+}{+}$ & $\stackrel{n}{\tilde{n}}$ & $\begin{array}{l}\stackrel{\circ}{1} \\
0 \\
0\end{array}$ \\
\hline 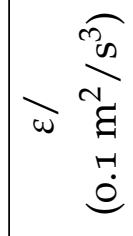 & $\begin{array}{l} \pm \\
\text { Z } \\
\text { i }\end{array}$ & $\begin{array}{l}0 \\
\stackrel{N}{N} \\
i\end{array}$ & 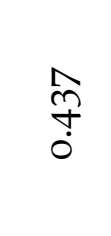 & $\begin{array}{l}\stackrel{9}{+} \\
\stackrel{i}{i}\end{array}$ & $\begin{array}{l}\stackrel{a}{a} \\
\stackrel{n}{m}\end{array}$ & \begin{tabular}{c}
$N$ \\
\multirow{+}{+}{} \\
$\dot{m}$
\end{tabular} \\
\hline$\Xi$ & 눙 & $\begin{array}{l}\circ \\
\stackrel{0}{0} \\
0\end{array}$ & กุ & $\stackrel{\infty}{m}$ & ثே. & $\stackrel{+}{\circ}$ \\
\hline 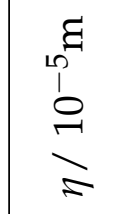 & $\begin{array}{c}\hat{n} \\
\hat{i}\end{array}$ & $\begin{array}{l}\text { Oे } \\
\text { ì }\end{array}$ & 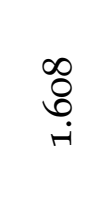 & $\begin{array}{l}\text { O } \\
\stackrel{+}{+} \\
\text {. }\end{array}$ & $\begin{array}{l}\hat{\sigma} \\
\delta \\
\dot{0}\end{array}$ & $\begin{array}{l}\hat{\hat{\alpha}} \\
\hat{0}\end{array}$ \\
\hline$\widetilde{\sim}$ & $\begin{array}{l}\text { aे } \\
\text { aे }\end{array}$ & 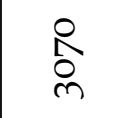 & $\begin{array}{l}\infty \\
\text { N } \\
\text { n }\end{array}$ & $\underset{7}{+}$ & $\begin{array}{l}8 \\
8 \\
\text { in }\end{array}$ & $\begin{array}{l}n \\
\infty \\
\infty \\
1\end{array}$ \\
\hline
\end{tabular}




\section{Appendix C: Fabrication of Free-standing Pt Nanowires for Use as Thermal Anemometry Probes in Turbulence Measurements}

This chapter is a reprint with permission of the paper "Fabrication of freestanding Pt nanowires for use as thermal anemometry probes in turbulence measurements", which is submitted to Nature Microsystems and Nanoengineering [1]. The author of the thesis performed the measurements in the wind tunnel, organised the production of a suitable operation circuit and analysed the corresponding data. The coauthors developed the probe and the production process, prototyped a measurement circuit, performed all measurements outside the Variable Density Turbulence Tunnel, organised the research, and wrote the manuscript.

[1] H. Le-The, C. Küchler, A. van den Berg, E. Bodenschatz, D. Lohse, and D. Krug. "Fabrication of free-standing Pt nanowires used as thermal anemometry probes for turbulence measurements". In: Nat. Microsystems Nanoeng. under consideration (2020) 
Free-standing Pt nanowires

Fabrication of free-standing Pt nanowires for use as thermal anemometry probes in turbulence measurements

Hai Le-The ${ }^{1,2,4}$, Christian Kuechler ${ }^{3,4}$, Albert van den Berg ${ }^{2,4}$, Eberhard Bodenschatz ${ }^{3,4}$, Detlef Lohse $^{1,4}$, Dominik Krug ${ }^{1.4}$

${ }^{1}$ Physics of Fluids Group, MESA+ Institute, University of Twente, Enschede 7522 NB, The Netherlands

${ }^{2}$ BIOS Lab-on-a-Chip Group, MESA+ Institute, University of Twente, Enschede 7522 NB, The Netherlands

${ }^{3}$ Max Planck Institute for Dynamics and Self-Organization, 37077 Göttingen, Germany

${ }^{4}$ Max Planck-University of Twente Center for Complex Fluid Dynamics

Correspondence: $\quad$ Hai Le-The (h.lethe@utwente.nl)

Dominik Krug (d.j.krug@utwente.nl)

KEYWORDS: Free-standing Pt nanowires, thermal anemometry probes, e-beam lithography, self-releasing, turbulence measurements 
Free-standing Pt nanowires

\begin{abstract}
We report a robust fabrication method for patterning free-standing Pt nanowires for the use as thermal anemometry probes for small-scale turbulence measurements. Using e-beam lithography, high aspect ratio Pt nanowires ( $\sim 300 \mathrm{~nm}$ width, $\sim 70 \mu \mathrm{m}$ length, $\sim 100 \mathrm{~nm}$ thickness) were patterned on the surface of oxidized silicon ( $\mathrm{Si}$ ) wafers. Combining precise wet etching processes with dry etching processes, these Pt nanowires have been successfully released freestanding between two silicon dioxide $\left(\mathrm{SiO}_{2}\right)$ beams supported on $\mathrm{Si}$ cantilevers. Moreover, the unique design of the bridge holding the device allowed to release the device gently without damaging the Pt nanowires. The total fabrication time was minimized by restricting the use of e-beam lithography to the patterning of the Pt nanowires while standard photolithography was employed for other parts of the devices. We demonstrate that the fabricated sensors are suitable for turbulence measurements when operated in a constant-current mode. A robust calibration between output voltage and fluid velocity was established over the velocity range from $0.5 \mathrm{~m}$ $\mathrm{s}^{-1}$ to $5 \mathrm{~m} \mathrm{~s}^{-1}$ in an $\mathrm{SF}_{6}$ atmosphere at a pressure of 2 bar and a temperature of $21^{\circ} \mathrm{C}$. The sensing signal from the nanowires showed negligible drift over a period of several hours. Moreover, we confirmed that the nanowires are able to withstand high dynamic pressures by testing them in air at room temperature velocities up to $55 \mathrm{~m} \mathrm{~s}^{-1}$.
\end{abstract}


Free-standing Pt nanowires

\section{INTRODUCTION}

Even today, fully resolved measurements of flow velocities in highly turbulent flows remain highly challenging. The difficulty is best illustrated by considering the non-dimensional Reynolds number $(R e)$, which measures turbulence intensity by relating the magnitudes of inertial and viscous forces acting in the flow. Accessing high $R e$ flows experimentally is important from a practical perspective as many engineering applications, such as the boundary layers on the hulls of ships and planes, fall into this regime. Moreover, measurements in high Re flows are also highly relevant to foster and to validate our theoretical understanding of the turbulence problem.

A hallmark of turbulence is the fact that 'eddying motions', i.e. seemingly random velocity fluctuations, across a wide range of scales contribute to the evolution of the flow. The range of spatial scales with $R e$ as $L / \eta \sim R e^{3 / 4}$, which renders the measurement challenge obvious ${ }^{1}$. If the largest scale $L$ is fixed, e.g. by the size of the lab facilities, high $R e$ can only be reached if the smallest scale $\eta$ is decreased in size. Typical sizes of $\eta$-and consequently the spatial resolution requirements - are in the order of micrometers. Also the temporal resolution is essential to resolve the short turnover timescales of such small eddies ${ }^{2,3}$. Especially in cases where flow structures are advected past the probe by a strong mean flow, frequency requirements can reach orders of $100 \mathrm{kHz}^{4}$.

To date, the best resolution and bandwidth characteristics are achieved using 'hotwire anemometry' (HWA), which is a proven technique with a long history ${ }^{5-8}$. Its measurement principle is based on the velocity dependent convective cooling of a heated wire element (with wire diameter $d$ ) that is placed in the fluid. The time varying cooling leads to changes in the electrical circuit (e.g. a change in wire resistance as the wire temperature changes if the heating current is kept constant), which can be calibrated to yield a fluid velocity measurement. The effective sensor size in HWA is given by the length $(\ell)$ of the wire. However, $\ell$ cannot be 
decreased arbitrarily. This is because a shorter wire length also increases the portion of the heat that leaves the wire via end-conduction, which is unwanted and detrimental to the measurement. This issue can only be overcome if shorter wires are also made thinner. Traditionally a minimum aspect ratio $\ell / d \leq 200$ has been used ${ }^{9}$, while more recently Hultmark et al. ${ }^{10}$ provided a refinement of this criterion. The conventional wire filaments with the best performance characteristics are produced from Wollaston wires by etching away part of the silver jacket. The sensing element is then formed by the exposed platinum $(\mathrm{Pt})$ wire for which minimum diameters of about $1 \mu \mathrm{m}$ can be achieved in this way. Pushing beyond this limit has proven very difficult despite significant efforts. For example, Willmarth \& Sharma produced wires with the length of $50 \mu \mathrm{m}$ using a Wollaston wire of $0.5 \mu \mathrm{m}$ in diameter ${ }^{11}$. However, given the relatively low aspect ratio, the performance of this design was hampered by end-conduction effects. Ligrani \& Bradshaw ${ }^{9}$ stuck to an aspect ratio of approximately 200 when designing the wires with a diameter of $0.625 \mu \mathrm{m}$, but with a minimum of $125 \mu \mathrm{m}$ the resulting wire length was still rather large. The need to decrease sensor sizes below this limitation initiated a push towards nanofabrication techniques. Early efforts by Löfdahl et al. ${ }^{12}$ yielded only moderate improvements as their probes featured a large sensing area. Jiang et al. ${ }^{13}$ employed microelectromechanical systems (MEMS) techniques to fabricate a poly-silicon thermal anemometry probe but the very good spatial resolution came at the price of significant endconduction losses in their case. End-conduction is also a problem for the multi-component hotwire probes $(50 \mu \mathrm{m} \times 6 \mu \mathrm{m} \times 2.7 \mu \mathrm{m})$ fabricated by Chen et al. ${ }^{14}$. Moreover, being fixed to a wall, these sensors are also not suitable for conventional turbulence measurements.

More recently, the development of the nanoscale thermal anemometry probe, termed NSTAP, at Princeton ${ }^{15-19}$ provided a breakthrough towards unprecedented small-scale resolution. Some noteworthy later developments, such as a micro fabricated multi-array probe that provides access to the full velocity gradient tensor ${ }^{20}$, or a specialized hotwire sensor for 
Free-standing Pt nanowires

measurements in cryogenic helium ${ }^{21}$ have been reported since. For completeness, it should also be mentioned that MEMS techniques have been employed to fabricate small-scale cantilevers for flow measurements 22,23 , but the measurement principle (beam deflection) is different in those cases., In terms of sensor size the NSTAP remains the state of the art to date. The production process of the NSTAP combines standard photolithography with a series of dryetching and wet-etching processes. The sensing element consists of a Pt wire, which is approximately $100 \mathrm{~nm}$ thick, while its width is still $1 \mu \mathrm{m}$. The latter is a limitation of the photolithography process but in part also a choice in order to enhance the convective heat transfer from the wire ${ }^{16}$. Note also that for a variant of the NSTAP, the q-NSTAP reported by Fan et al. ${ }^{18}$, electron-beam lithography is employed. This reduces the width of the wire to between $600 \mathrm{~nm}$ and $800 \mathrm{~nm}$. However, with a length of only $10 \mu \mathrm{m}$, the q-NSTAP is designed to measure humidity and is not suited for anemometry. Even with these reduced wire dimensions, the authors report issues regarding the structural integrity of the sensor due to internal stresses originating from wet etching of silicon dioxide $\left(\mathrm{SiO}_{2}\right)$ to release the wire.

In this paper, we report a robust method for fabrication of free-standing Pt nanowires (300 $\mathrm{nm}$ width, $100 \mathrm{~nm}$ thickness) with length as long as $70 \mu \mathrm{m}$ for the use as thermal anemometer probes for turbulence measurements. We describe how by combining e-beam lithography with precise wet etching processes and dry etching processes, Pt nanowires have been successfully fabricated free-standing between two silicon dioxide $\left(\mathrm{SiO}_{2}\right)$ beams supported on Si cantilevers. We further confirm that the fabricated nanowires are capable and sufficiently robust to measure the velocity of turbulent flows even at large fluid densities. We tested this in the Max Planck Variable Density Turbulence Tunnel (VDTT) with pressurized $\mathrm{SF}_{6}$ as working fluid as well as in an air flow with velocities up to $5 \mathrm{~m} \mathrm{~s}^{-1}$ without damaging the wires. 
Free-standing Pt nanowires

\section{RESULTS AND DISCUSSION}

Figure 1 presents an overview over the processing sequence for the fabrication process of a device featuring a free-standing Pt nanowire. Further details on the dimensions of the structure are provided in the Supplementary information(Figure S1). We elaborate on individual fabrication steps in the following. Further details and the specific processing parameters employed are provided in the Materials and Methods section.

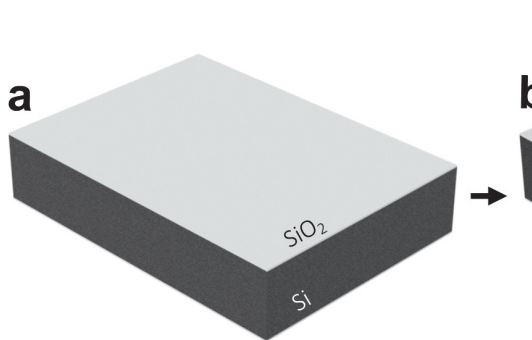

Oxidized Si substrate
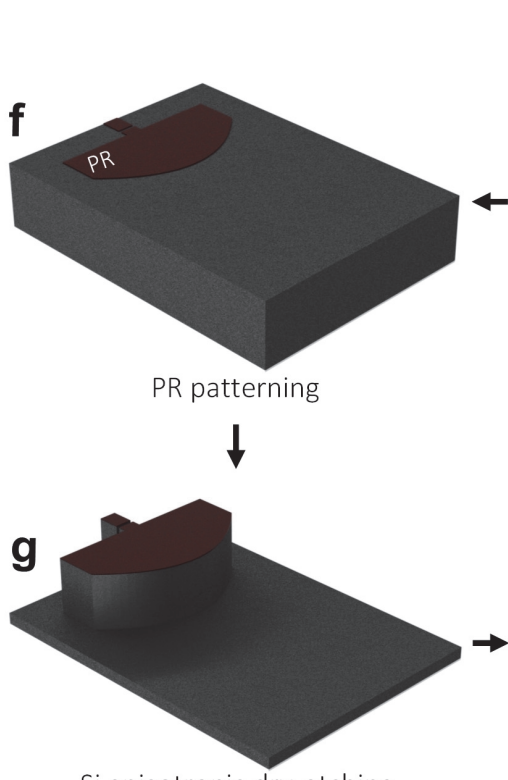

Si anisotropic dry-etching

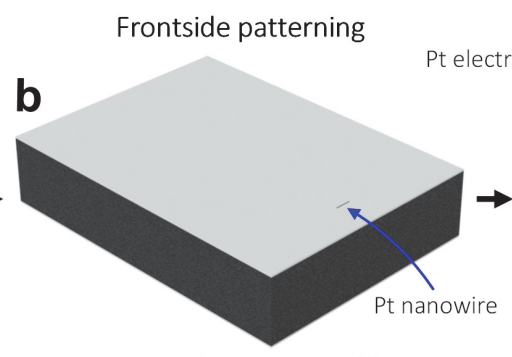

Pt nanowire patterned by e-beam lithography

Backside patterning

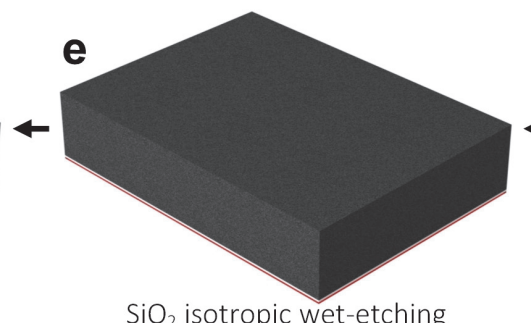

$\mathrm{SiO}_{2}$ isotropic wet-etching using BHF solution

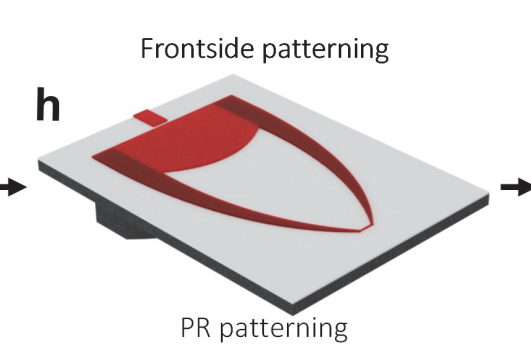

PR patterning

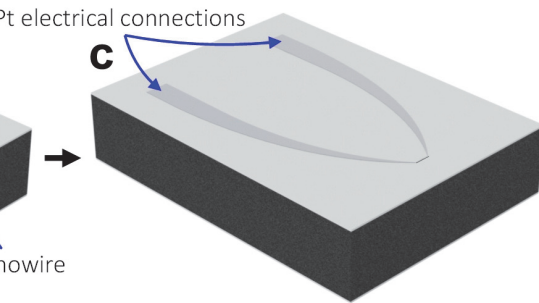

Pt connections patterned by photolithography

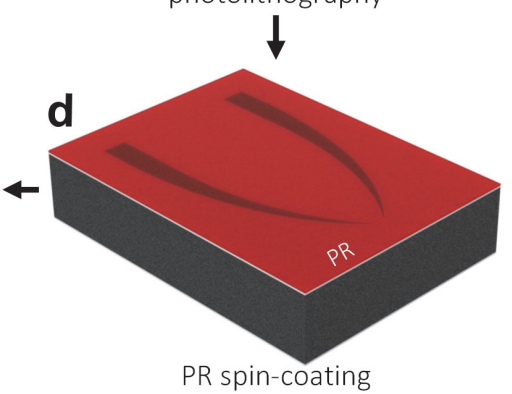

PR spin-coating

Free-standing PR line with Pt nanowire

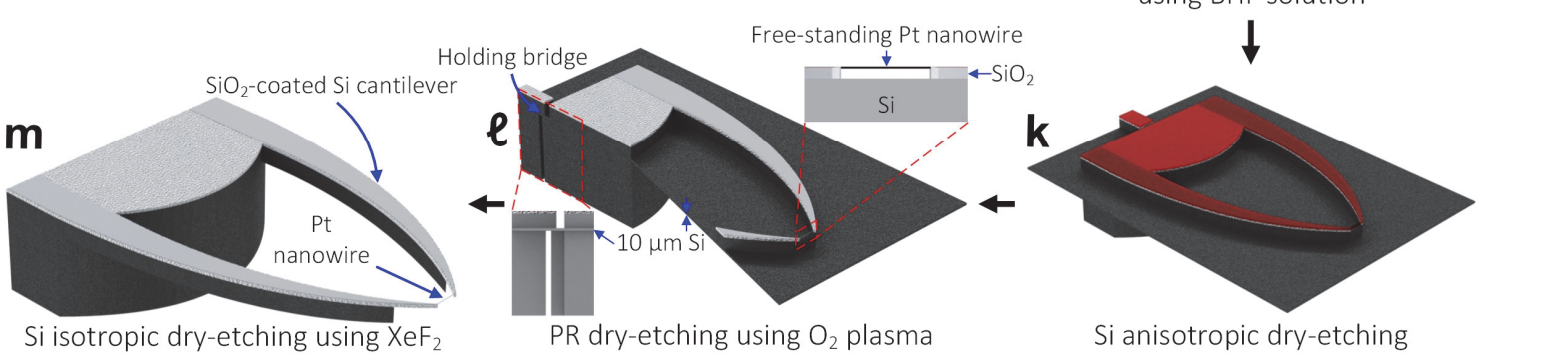

Figure 1 Fabrication process for patterning free-standing Pt nanowires. (a) Wet thermal oxidation of a Si wafer. (b) Patterning a Pt nanowire using e-beam lithography. (c) Frontside 
Free-standing Pt nanowires

patterning Pt connections to the Pt nanowire using standard photolithography. (d) Spin-coating of photoresist (PR) on the frontside of the patterned wafer, and (e) wet etching of $\mathrm{SiO}_{2}$ layer on its backside using a BHF solution. (f) Backside patterning a PR structure of the device base, followed by (g) deep dry etching of Si. (h) Frontside patterning a PR structure of the support cantilevers. (i) Wet etching of $\mathrm{SiO}_{2}$ using a BHF solution, resulting in a free-standing PR line with the Pt nanowire. (k) Dry etching of $\mathrm{Si}$, followed by $(\boldsymbol{\ell})$ dry etching of PR using $\mathrm{O}_{2}$ plasma at low power. (m) Isotropic dry etching of $\mathrm{Si}$ using $\mathrm{XeF}_{2}$ for self-releasing of the device.

\section{Patterning Pt nanowires using electron beam lithography}

An e-beam lithography (EBL) system operating at $100 \mathrm{kV}$ (Raith EBPG 5150, Raith GmbH, Germany) was used to pattern Pt nanowires on the surface of oxidized Si wafers (Figure 1b). These wafers were prepared by wet thermal oxidation of conventional (100) 4 -inch silicon (Si) wafers $(385 \mu \mathrm{m}$ thick, Okmetic, Finland) (Figure 1a). Prior to the sputtering of Pt, a thin titanium (Ti) layer of $\sim 13 \mathrm{~nm}$ thickness was sputtered in order to improve the adhesion of these patterned Pt nanowires. The choice of Ti for the adhesion layer is beneficial here because it can be easily removed together with $\mathrm{SiO}_{2}$ layer in a buffered hydrofluoric acid (BHF) solution, thus leaving free-standing pure Pt nanowires. Figure 2 shows the high-resolution scanning electron microscopy (HR-SEM) images of a Pt nanowire fabricated on the surface of an oxidized Si wafer. A well-defined Pt nanowire was obtained with dimensions matching the specifications ( $\sim 300 \mathrm{~nm}$ width, $\sim 70 \mu \mathrm{m}$ length, $\sim 100 \mathrm{~nm}$ thickness). The pattern was expanded slightly at the wire tips to facilitate the electrical connection 

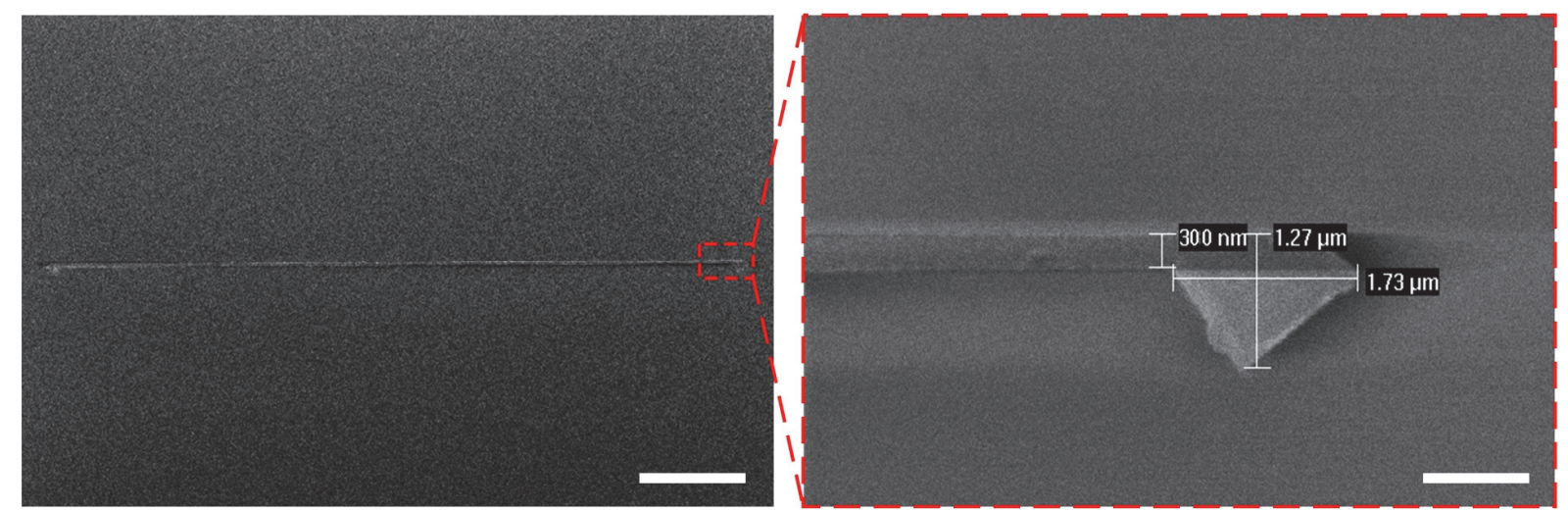

Figure 2 Top-view HR-SEM image (scale bar: $10 \mu \mathrm{m}$ ) of a Pt nanowire patterned on the surface of an oxidized Si wafer, with a close-up image of the tip of the wire which is expanded slightly to facilitate the connection with the Pt micropattern (scale bar: $1 \mu \mathrm{m}$ ).

\section{Patterning Pt connections to the Pt nanowires}

For the electrical connection to the Pt nanowire, Pt micropatterns (termed Pt connections) were fabricated by combining standard photolithography with a lift-off process (Figure 1c). Figure 3 shows the optical microscopy images of Pt connections patterned on the surface of an oxidized Si wafer. It should be noted that the precision of the overlay of the Pt connections with the Pt nanowire is crucial in this step as any misalignment between these structures can disrupt the electrical connection with the Pt nanowire.
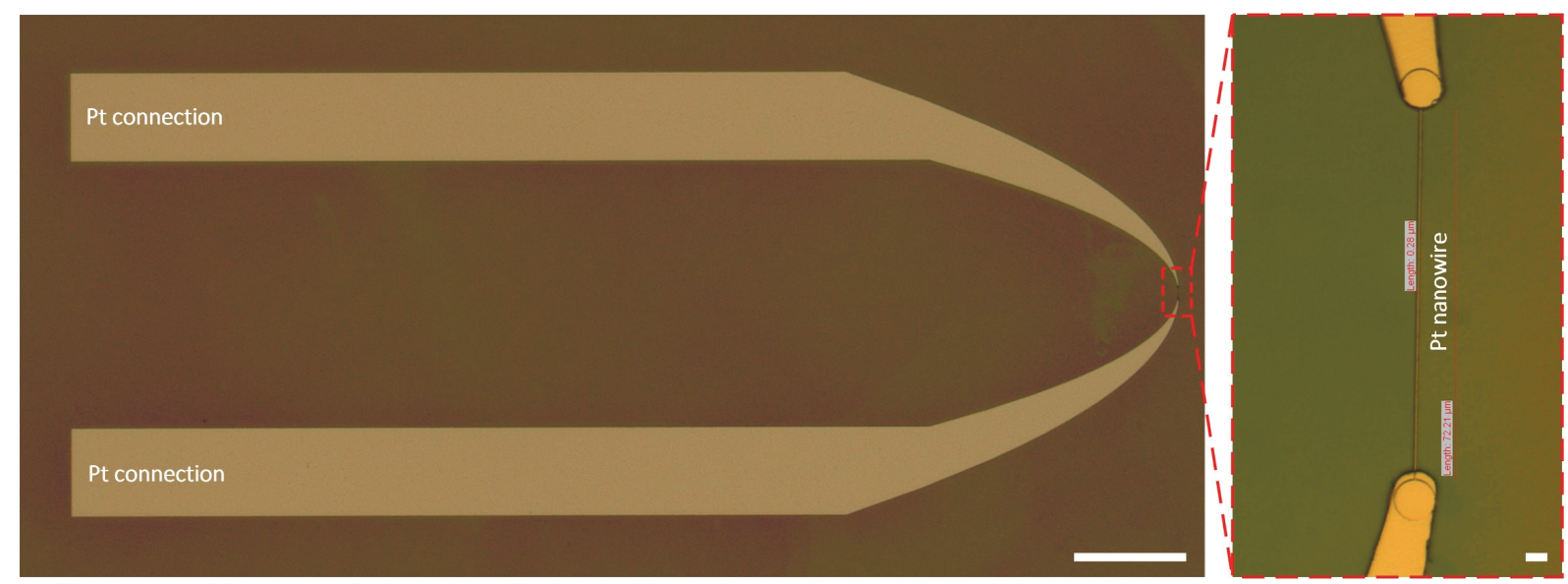

Figure 3 Optical microscopy image (scale bar: $500 \mu \mathrm{m}$ ) of Pt connections, with a close-up image at the Pt nanowire location (scale bar: $5 \mu \mathrm{m}$ ). 


\section{Backside patterning of the device base using dry etching of Si}

Prior to the backside patterning of the wafer, its frontside was covered with a PR layer (Figure 1d). The wafer was then immersed in a BHF solution in order to completely remove the $\mathrm{SiO}_{2}$ layer on the backside (Figure 1e), while the $\mathrm{SiO}_{2}$ layer on the frontside containing the patterned Pt structures remained protected by the PR coating.

Subsequently, a PR structure of the device base was patterned on the backside of the wafer using a standard photolithography process (Figure 1f). The patterned PR structure was hardbaked at $120^{\circ} \mathrm{C}$ for 10 min to harden the PR areas before conducting the etching of $\mathrm{Si}$ in an inductively coupled plasma (ICP) deep reactive ion etching (DRIE) instrument (SPTS Pegasus system, UK), using the standard Bosch process (Figure 1g). Figure 4 shows the HR-SEM images of the device base after the dry etching process. It is worth mentioning that a negatively tapered profile was obtained after the deep Si etching. This needs to be taken into account when

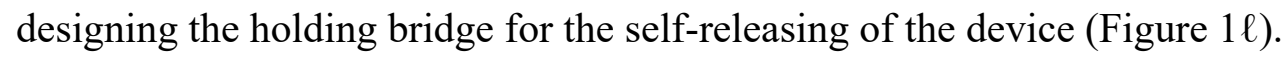

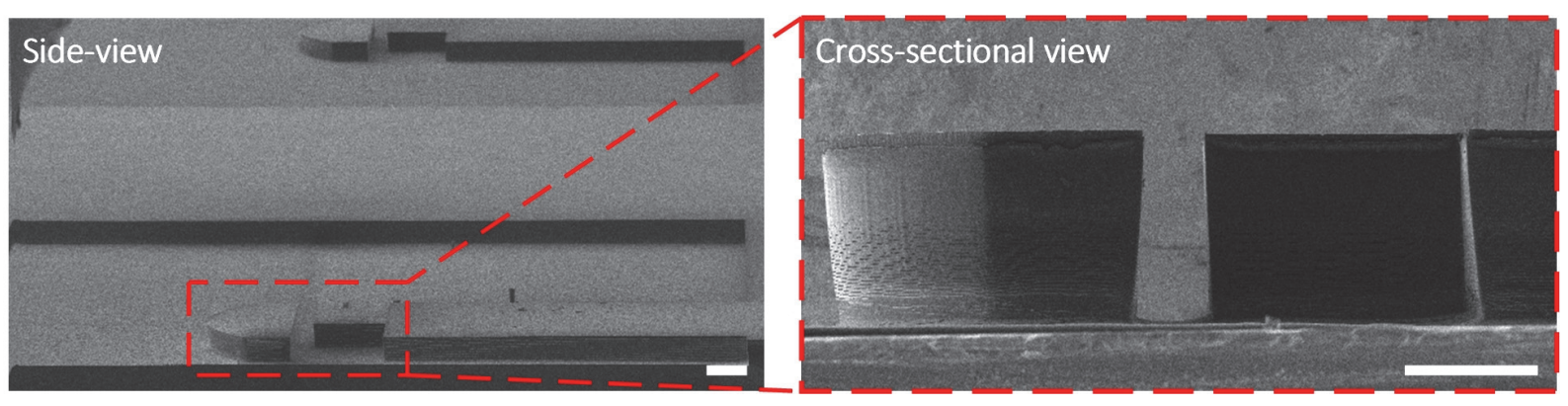

Figure 4 Side-view and cross-sectional HR-SEM images (scale bar: $200 \mu \mathrm{m}$ ) of backside patterning of the device base using dry etching of Si.

\section{Frontside patterning of the device}

Figure 5 shows the optical microscopy images of a PR structure patterned on top of the Pt structure. Also in this case, the alignment of the patterned PR structure with the Pt structure needs to be precise so that the PR structure completely covers the Pt structure, especially at the Pt nanowire location where it is covered by a PR line, as shown in the close-up image (Figure 
5). This ensures that the Pt structure is not damaged during the subsequent patterning of the cantilevers by wet etching and dry etching processes (Figures $1 \mathrm{i}$ and $1 \mathrm{k}$ ).

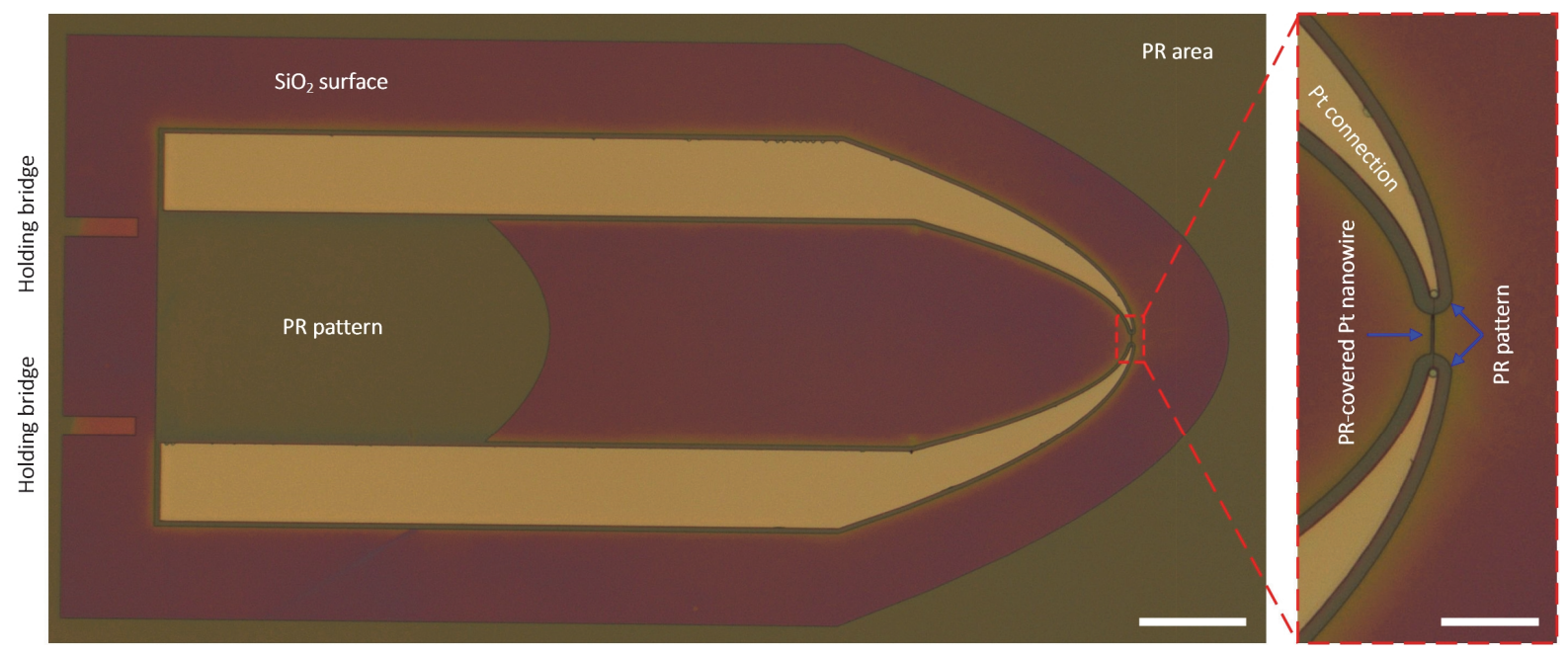

Figure 5 Optical microscopy image (scale bar: $500 \mu \mathrm{m}$ ) of a PR structure patterned on top of the Pt structure, with a close-up image at the Pt nanowire location (scale bar: $100 \mu \mathrm{m}$ ).

For releasing the PR line, the patterned wafers were immersed in a BHF solution for $30 \mathrm{~min}$. As a result, the $\mathrm{SiO}_{2}$ under the PR line was etched, thus leaving the free-standing PR line with the Pt nanowire stuck to it (Figures $1 \mathrm{i}$ and $6 \mathrm{~b}$ ). Since both PR and Si are hydrophobic any liquid trapped between the PR line and the Si surface was removed quickly and easily when spindrying the wafers. Importantly, this resulted in no damage to the free-standing PR line supporting the Pt nanowire.

Figure 6a shows the side-view HR-SEM image of the support cantilevers after dry etching of Si from the frontside of the wafer. This etching process needs to be stopped when the thickness of the remaining Si membrane (Figure $1 \mathrm{k}$ ) is down to approximately $10 \mu \mathrm{m}$. Etching through the Si-layer can lead to a leak of cooling gas from the backside, thus terminating the etching process. Crucially, further etching without cooling can result in burning of the Pt line, and hence a breaking of the Pt nanowire. 

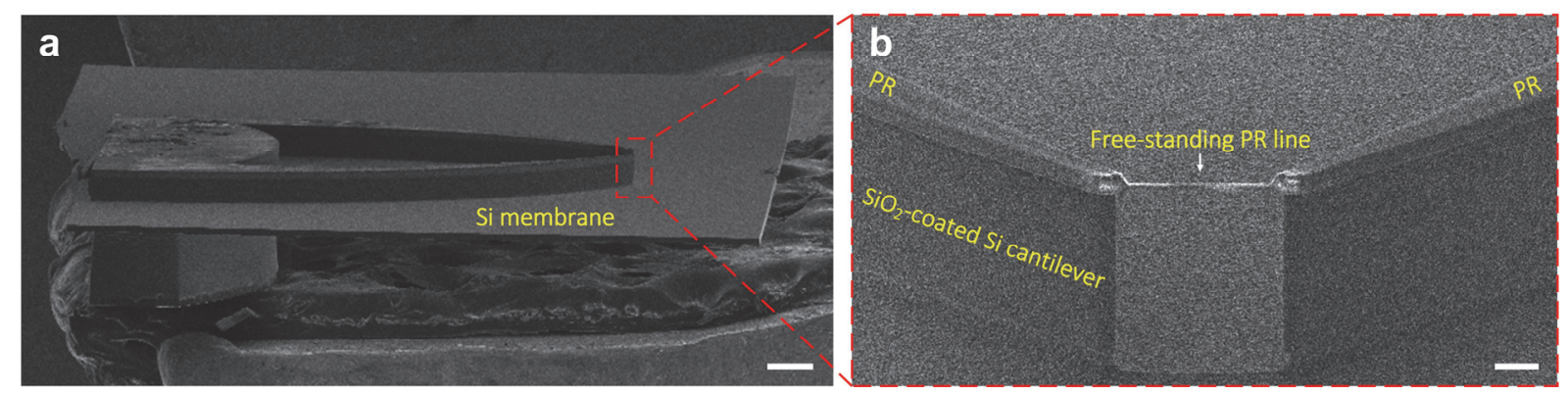

Figure 6 (a) Side-view HR-SEM image (scale bar: $500 \mu \mathrm{m}$ ) support cantilevers after dry etching of Si from the frontside of the wafer. Note that the slight damage visible on the top surface of the device base was caused by handling during the SEM inspection. (b) Crosssectional HR-SEM image (scale bar: $20 \mu \mathrm{m}$ ) of a free-standing PR line with the Pt nanowire.

To remove the PR covering the $\mathrm{Pt}$ nanowire, reactive $\mathrm{O}_{2}$ plasma etching was used (Figure 1थ). This needs to be done gently at low power to avoid burning the PR line, and thereby breaking the Pt nanowire. The removal of PR was conducted before releasing the device because it turned out that the PR line became brittle after the dry etching process (Figure 1k). This resulted in frequent damage of the PR line during the releasing which then also affected the Pt nanowire.

\section{Isotropic dry etching of $\mathrm{Si}$ using $\mathrm{XeF}_{2}$}

Figure 7 shows the HR-SEM images of a fabricated device consisting of a Pt nanowire freestanding between two $\mathrm{SiO}_{2}$ beams supported on Si cantilevers (Figure 1m). After isotropic dry etching of $\mathrm{Si}$ in $\mathrm{XeF}_{2}$, the remaining $\mathrm{Si}$ membrane was completely etched, forming two freestanding $\mathrm{SiO}_{2}$-coated $\mathrm{Si}$ cantilevers (Figure $7 \mathrm{~b}$ ). It should be noted that the $\mathrm{Si}$ underneath the $\mathrm{Pt}$ nanowire as well as the $\mathrm{Si}$ at the tip of two cantilevers were also etched, thus resulting in the $\mathrm{Pt}$ nanowire free-standing on $\mathrm{SiO}_{2}$ beams (Figure 7c).

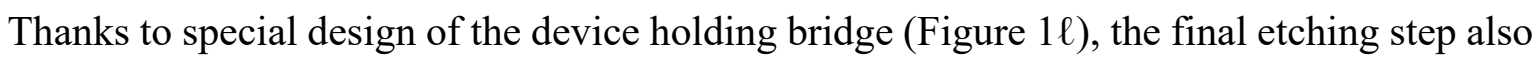
served to self-release the device from the wafer. The holding bridge also has a remaining Si 
Free-standing Pt nanowires

layer of $\sim 10 \mu \mathrm{m}$ and is thus etched away in $\mathrm{XeF}_{2}$. This self-releasing procedure has proven necessary and important since it appeared that breaking the device led to frequent failures of the Pt nanowire (presumably due to the vibrations of the cantilevers). As confirmed in the closeup images, the resulting free-standing Pt nanowire has a width of $\sim 300 \mathrm{~nm}$ and a length of $\sim 70$ $\mu \mathrm{m}$.
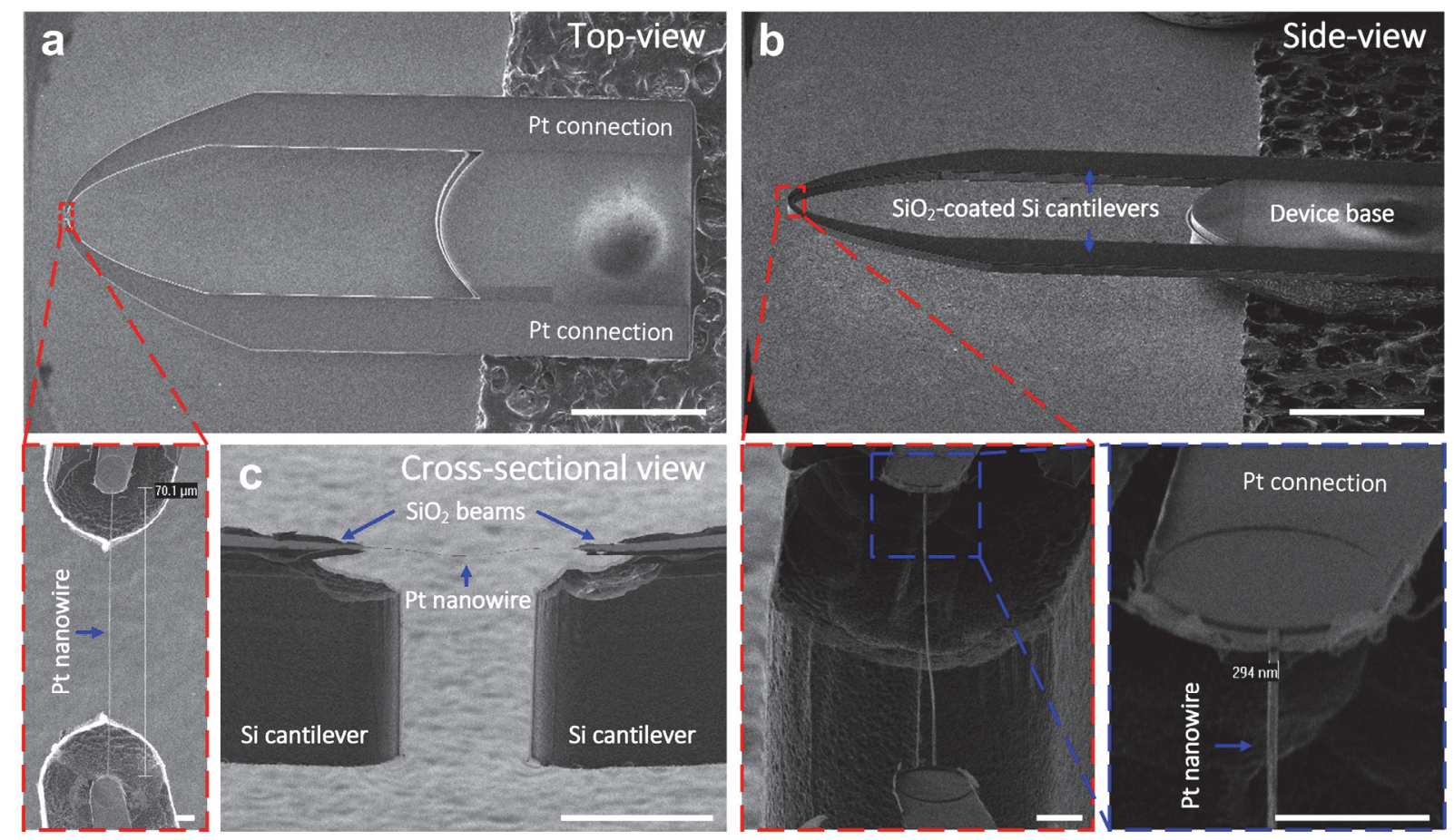

Figure 7 (a) Top-view and (b) side-view HR-SEM images (scale bar: $1 \mathrm{~mm}$ ) with close-up images (scale bar: $5 \mu \mathrm{m}$ ) of a fabricated device consisting of a free-standing Pt nanowire $(\sim 300$ nm width, $\sim 70 \mu \mathrm{m}$ length, $\sim 100 \mathrm{~nm}$ thickness). (c) Cross-sectional HR-SEM image (scale bar: $50 \mu \mathrm{m}$ ) of a fabricated $\mathrm{Pt}$ nanowire hanging between two $\mathrm{SiO}_{2}$ beams supported on $\mathrm{Si}$ cantilevers.

\section{Performance of the fabricated devices used as thermal anemometer probes}

A typical initial cold resistance for the nanowire is $820 \Omega$, but this value was seen to drop significantly when the wire was first heated up. Annealing the nanowire with currents of $\sim 1$ $\mathrm{mA}$ reduced the resistance to $R_{w} \approx 740 \Omega$, and this value was found to be stable over repeated 
Free-standing Pt nanowires

heating cycles with comparable currents. By measuring the wire resistance in a temperature controlled environment we determined the temperature coefficient of resistivity to be $\alpha 20^{\circ} \mathrm{C}=$ $0.0021 \mathrm{~K}^{-1}$.

(a)

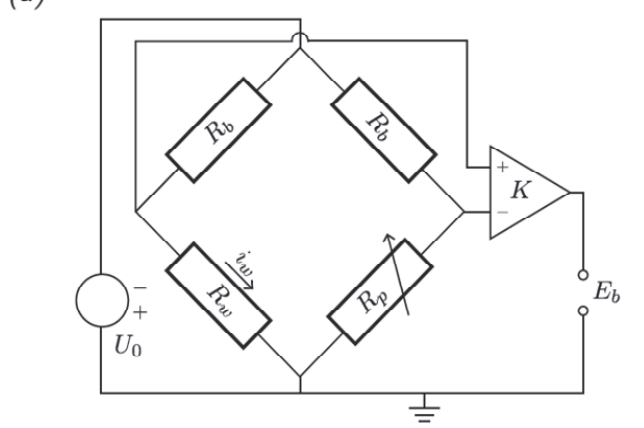

(b)

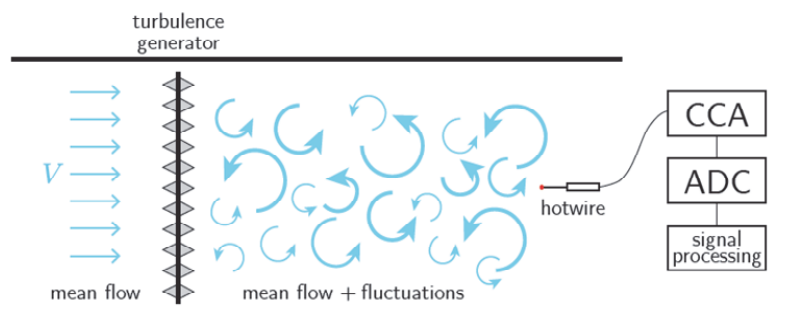

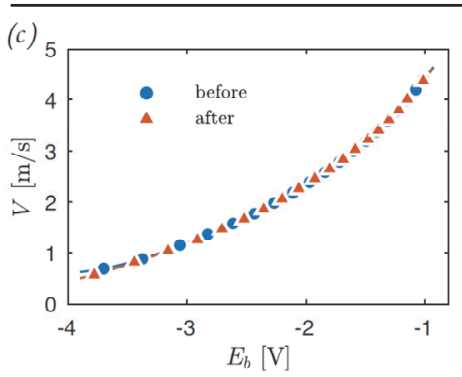
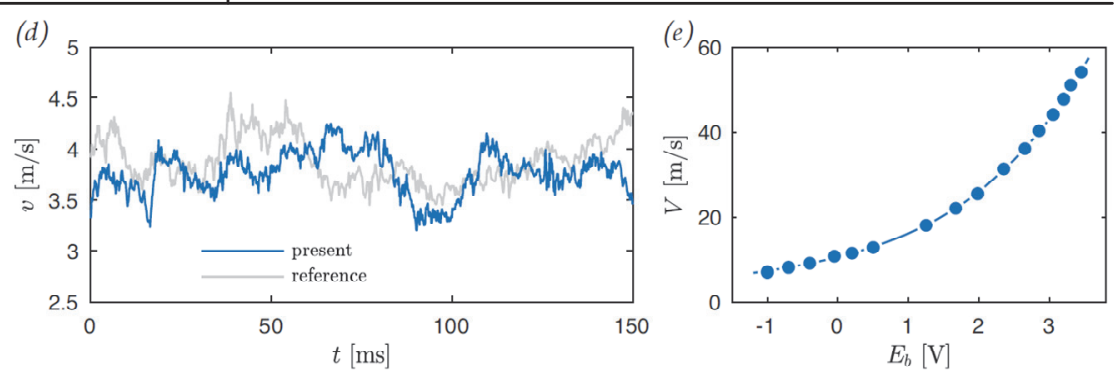

Figure 8 (a) Sketch of the CCA circuit. (b) Measurement setup in the VDTT. (c) Calibration results in the VDTT taken before and after a measurement campaign. (d) Measured velocity time trace compared to results from a reference probe located nearby. (e) Calibration results in air at room temperature.

The nanowires were operated in a bridge circuit (see figure 8a) and tested in the Max Planck Variable Density Turbulence tunnel (VDTT) in Göttingen ${ }^{24}$ in a gaseous sulfur hexafluoride (SF6) environment at extremely high Reynolds numbers (see schematic in figure 8b). Further details of the setup and operating conditions are given in the Materials and Methods section. In order to calibrate the sensor output voltage $E_{b}$ against the fluid velocity, a time average of $E_{b}$ was recorded for several settings of the tunnel velocity $V$ in non-turbulent conditions. In order to gauge a potential drift of the bridge voltage, the calibration was performed both before and after a measurement campaign that spanned several hours. The calibration results are presented 
Free-standing Pt nanowires

in Figure 8(c). There is a clear and monotonic trend between $E_{b}$ and $V$ that can be captured very accurately over the full velocity range by fitting a fourth-order polynomial (indicated by the lines), which is a standard procedure for hotwire measurements ${ }^{25}$. Importantly, calibration results before and after the measurement campaign are almost indistinguishable, indicating that the drift of the sensor is negligible over an operation period of several hours. As an additional validation, we compare the time trace of the measured fluctuating velocity $v(t)$ to results obtained using a standard probe (length $450 \mu \mathrm{m}$, diameter $2.5 \mu \mathrm{m}$, DANTEC custom design) as a reference in Figure $8(\mathrm{~d})$. The probes were not located at the exact same location such that a one-to-one comparison is not possible. It does, however, become clear at least qualitatively that the present wire captures velocity fluctuations across the full range of magnitudes and time scales consistently with the reference. Further, there were no issues operating the wires at pressures up to 15 bar in $\mathrm{SF}_{6}$, at which the gas density is more than $1 / 10^{\text {th }}$ that of water at room temperature. To test whether the wires also perform well in other fluids and at larger flow speeds, we additionally operated the wire in air at room temperature. In this case the flow was generated by pressurized air exiting a nozzle. Also here, the data can be very well represented by a monotonically increasing fourth-order polynomial across the full range of $5 \mathrm{~m} \mathrm{~s}^{-1} \leq V \leq 55$ $\mathrm{m} \mathrm{s}^{-1}$. The wire was able to withstand the dynamic pressure at the highest velocities without any problems (Figure 8e).

\section{CONCLUSION}

In summary, we report a robust fabrication method, combining e-beam lithography with wet etching and dry etching processes, for patterning free-standing Pt nanowires used as thermal anemometer probes for turbulence measurements. With precise control of the dry etching processes, Pt nanowires ( $\sim 300 \mathrm{~nm}$ width, $\sim 100 \mathrm{~nm}$ thickness) with a length of $70 \mu \mathrm{m}$ have been successfully released free-standing between two $\mathrm{SiO}_{2}$ beams that are supported on $\mathrm{Si}$ cantilevers. 
Free-standing Pt nanowires

A critical aspect is the design of the holding bridge, which ensures a safe and gently release of the device without damaging the wires. Further, limiting the use of e-beam lithography to the patterning of the Pt nanowires renders the process cost and time efficient. These benefits far outweigh the additional complication arising from the resulting need to align e-beam and optical lithography patterns with high accuracy.

The operational tests have confirmed that the wires are suitable for turbulence measurements in different working media and at a high dynamic pressures. For this purpose the nanowire design presented here holds a lot of promise regarding several aspects: (i) A more slender wire allows to use shorter wire lengths without being compromised by end-conduction effects; (ii) Smaller sensing elements are expected to improve the frequency response of the anemometer even if the wire is operated in a constant temperature mode ${ }^{26}$; (iii) Due to its very small thermal inertia, the wires can yield sufficient frequency resolution for many flow cases even when operated in constant-current mode. This eliminates the need for a feedback loop, thereby simplifying the circuitry significantly; (iv) The quasi circular shape of the sensing element is expected to avoid an unwanted pitch sensitivity of the sensor. We aim to explore and quantify these benefits in the future in an effort to push the limits for highly resolved high Re turbulence measurements.

\section{MATERIALS AND METHODS}

\section{Wet thermal oxidation of Si wafers}

Conventional (100) 4-inch Si wafers (385 $\mu$ m thick, Okmetic, Finland) with a thick thermal oxide layer of approximately $2 \mu \mathrm{m}$ were prepared by wet thermal oxidation (Figure 1a). Prior to the wet thermal oxidation process, all the $\mathrm{Si}$ wafers were cleaned to prevent crosscontamination $^{27}$. Subsequently, the Si wafers were loaded into a high temperature tube furnace (Model 287, TEMPRESS), using a quartz carrier to implement the wet oxidation at $1150^{\circ} \mathrm{C}$ for 
Free-standing Pt nanowires

$12 \mathrm{~h}$. During the oxidation process, the flow rate of a mixture of water vapor and nitrogen gas was fixed at $2 \ell \min ^{-1}$. The ramping and cooling rates were set at $10^{\circ} \mathrm{C} \min ^{-1}$ and $7^{\circ} \mathrm{C} \min ^{-1}$, respectively.

\section{Patterning Pt nanowires using electron beam lithography}

Prior to the e-beam writing, positive resist $\left(\mathrm{NANO}^{\mathrm{TM}}\right.$ 950PMMA Series Resists in Chlorobenzene, MicroChem, US) was spin-coated over the surface of the oxidized Si wafers at $2500 \mathrm{rpm}$ for $45 \mathrm{~s}$, followed by baking at $165^{\circ} \mathrm{C}$ for $2 \mathrm{~min}$. Subsequently, an e-beam lithography (EBL) system with a 100-kV (Raith EBPG 5150, Raith GmbH, Germany) was used to write the nanowire pattern into the resist layer. The written wafers were then developed in a developer solution (MIBK-IPA mixture) in $90 \mathrm{~s}$, followed by rinsing with deionized (DI) water using a quick dump rinser and spin-drying with nitrogen $\left(\mathrm{N}_{2}\right)$.

A titanium (Ti) layer of $\sim 13 \mathrm{~nm}$ and a platinum (Pt) layer of $\sim 100 \mathrm{~nm}$ were sputtered over the patterned wafers using an ion-beam sputtering system (home-built T'COathy system, MESA+, NanoLab) ${ }^{28}$. The sputtering processes were performed at $200 \mathrm{~W}$ and at a pressure of $6.6 \times 10^{-3}$ mbar which was adjusted using an argon (Ar) flow. Subsequently, the wafers were immersed in acetone with sonication to perform the lift-off process. After rinsing the wafers with DI water and spin-drying with $\mathrm{N}_{2}$, the fabrication of Pt nanowires patterned on the surface of the oxidized Si wafers was finished (Figure 1b).

\section{Patterning Pt connections to the Pt nanowires}

A positive photoresist (PR) layer (OiR 907-17i, Fujifilm, Japan) was spin-coated over the wafer surface at $4000 \mathrm{rpm}$ for $45 \mathrm{~s}$, followed by baking at $95^{\circ} \mathrm{C}$ for 1 min. A photo-mask made of quartz containing inverted chromium $(\mathrm{Cr})$ patterns connected to the patterned Pt nanowires was fabricated in-house by using a mask-making system (DWL 2000 Laser Lithography System, 
Free-standing Pt nanowires

Heidelberg Instruments, Germany). The exposure process was performed by using a mask alignment system (EVG620, EV Group, Austria) for $5 \mathrm{~s}$ at an intensity of $12 \mathrm{~mW} \mathrm{~cm}{ }^{-2}$ in hard contact mode. Thereafter, the wafers were post-baked at $120^{\circ} \mathrm{C}$ for 1 min, followed by developing in a OPD4246 solution for 1 min, rinsing with DI water, and drying with $\mathrm{N}_{2}$. A Ti layer of $\sim 6 \mathrm{~nm}$ and a Pt layer of $\sim 100 \mathrm{~nm}$ were sputtered over the patterned wafers using the T'COathy system. The lift-off process was conducted in acetone with sonication, followed by rinsing the wafers with DI water. After spin-drying with $\mathrm{N}_{2}$, the fabrication of Pt connections to the Pt nanowires was completed (Figure 1c).

\section{Backside etching of the thermal oxide layer}

The patterned surface of the oxidized Si wafers was covered with a PR layer (OiR 908-35, Fujifilm, Japan) by spin-coating at $2000 \mathrm{rpm}$ for $45 \mathrm{~s}$, followed by baking at $95^{\circ} \mathrm{C}$ for $3 \mathrm{~min}$ (Figure 1d). The wafers were then immersed in a buffered hydrofluoric acid (BHF) solution for $30 \mathrm{~min}$ to remove the $\mathrm{SiO}_{2}$ layer completely (etch rate of $\sim 68 \mathrm{~nm} \mathrm{~min}^{-1}$ ) on their backside (Figure 1c).

\section{Backside patterning of the device base using dry etching of Si}

After removing the PR layer in acetone, cleaning with DI water and drying with $\mathrm{N}_{2}$ gas, the backside of the wafers was spin-coated with a PR layer (OiR 908-35, Fujifilm, Japan) at 2000 rpm for $45 \mathrm{~s}$, followed by baking at $95^{\circ} \mathrm{C}$ for $3 \mathrm{~min}$. A photo-mask containing a $\mathrm{Cr}$ pattern of the device base was used for the exposure process, which was performed by using the mask alignment EVG620 system for $15 \mathrm{~s}$ at an intensity of $12 \mathrm{~mW} \mathrm{~cm}{ }^{-2}$ in hard contact mode. The alignment with the frontside Pt structures was performed using the bottom alignment with cross-hair mode. Thereafter, the wafers were post-baked at $120^{\circ} \mathrm{C}$ for $1 \mathrm{~min}$, followed by developing in the OPD4246 solution for 3 min, rinsing with DI water, and drying with $\mathrm{N}_{2}$. 
Free-standing Pt nanowires

Subsequently, the wafers were baked at $120^{\circ} \mathrm{C}$ for $10 \mathrm{~min}$ to harden the remaining PR areas for further backside etching of the Si (Figure 1f).

The etching of Si was conducted in an inductively coupled plasma (ICP) deep reactive ion etching (DRIE) instrument (SPTS Pegasus system, UK), using the standard Bosch process with 105 cycles ( $0.6 \mathrm{~s}$ deposition of $\mathrm{C}_{4} \mathrm{~F}_{8}, 1.75 \mathrm{~s}$ etching of $\mathrm{Si}$ by $\mathrm{SF}_{6}$ ) (Figure $1 \mathrm{~g}$ ). After the deep Si etching, the wafers were immersed in a $99 \%$ nitric acid $\left(\mathrm{HNO}_{3}\right)$ solution for 30 min to completely remove the PR layer and any other residues.

\section{Frontside patterning of the device}

Subsequently, the wafers were flipped and their frontside was spin-coated with a positive PR layer (OiR 907-17i, Fujifilm, Japan) at $4000 \mathrm{rpm}$ for $45 \mathrm{~s}$, followed by baking at $95^{\circ} \mathrm{C}$ for 1 min. A photo-mask containing Cr pattern of support cantilevers was used for the exposure process was performed by using the mask alignment EVG620 system for $5 \mathrm{~s}$ at an intensity of $12 \mathrm{~mW} \mathrm{~cm}{ }^{-2}$ in hard contact mode. The wafers were then post-baked at $120^{\circ} \mathrm{C}$ for $1 \mathrm{~min}$, followed by developing in the OPD4246 solution for $1 \mathrm{~min}$, rinsing with DI water, and drying with $\mathrm{N}_{2}$. Subsequently, the wafers were baked at $120^{\circ} \mathrm{C}$ for $10 \mathrm{~min}$ to harden the PR layer (Figure 1h).

\section{Releasing of the PR line with the Pt nanowire}

The patterned wafers were then immersed in the BHF solution for $30 \mathrm{~min}$ to remove the unprotected $\mathrm{SiO}_{2}$ layer completely. Since the PR line covering the Pt nanowire at the tip of the cantilevers has a small width of approximately $3 \mu \mathrm{m}$, etching in the BHF solution for $30 \mathrm{~min}$ resulted in a complete removal of $\mathrm{SiO}_{2}$ under the PR line and $\mathrm{Ti}$ under the Pt nanowire. As a result, the PR line with the Pt nanowire stuck to it got released in this step (Figure 1i). 
Free-standing Pt nanowires

Patterning support cantilevers using dry etching of Si

The wafers were then etched in the SPTS Pegasus system using the fine-etching process with 90 cycles (Figure 1k) until the remaining Si layer reached a thickness of approximately $10 \mu \mathrm{m}$.

\section{Etching of the PR line using $\mathrm{O}_{2}$ plasma}

To remove the PR covered the Pt nanowire, oxygen $\left(\mathrm{O}_{2}\right)$ plasma etching was performed in a parallel plate reactive ion etching system (home-built TEtske system, MESA+, NanoLab) at wafer-level, $10 \mathrm{mTorr}$, and $25 \mathrm{~W}$ for $20 \mathrm{~min}$. A low power etching was used in order to not

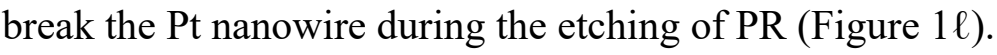

\section{Isotropic etching of $\mathrm{Si}$ using $\mathrm{XeF}_{2}$}

For the final patterning of the cantilevers and for releasing the devices, the wafers were put in a gas phase Xactix $\mathrm{XeF}_{2} \mathrm{E} 1$ system (etching time per cycle: $30 \mathrm{~s}$, temperate: $35^{\circ} \mathrm{C}$, pressure: 3000 mTorr), so that the $\mathrm{Si}$ was isotropically etched by xenon difluoride ( $\mathrm{XeF}_{2}$, etching rate of $\sim 1 \mu \mathrm{m}$ ) (Figure $1 \mathrm{~m})$. This resulted in an etching through of the remaining Si layer, thus forming two free-standing $\mathrm{SiO}_{2}$-coated $\mathrm{Si}$ cantilevers. The $\mathrm{Si}$ underneath the Pt nanowire and the $\mathrm{Si}$ at the tip of two cantilevers were also etched, thus resulting in the Pt nanowire free-standing on $\mathrm{SiO}_{2}$ beams. The device was also self-released after this etching step thanks to the special design of the device holding bridge.

\section{Electrical connection to the device using a silver conductive glue}

For the electrical connection, the fabricated device was mounted on the prongs of a commercial probe holder (Dantec Dynamics A/S, Denmark) using a silver conductive glue (Figure S2). In order to cure the glue, the device mounted probe was baked in an oven at $120^{\circ} \mathrm{C}$ for at least 15 min. 
Free-standing Pt nanowires

\section{Testing the fabricated devices used as thermal anemometer probes}

To operate the nanowire, we used a constant current anemometer (CCA) circuit as sketched in Figure 8a. Here, the device was placed in a bridge that features large ballast resistances $R_{b}=12$ $\mathrm{k} \Omega$ at the top of both arms. Since $R_{b} \gg R_{w}$, this ensures that the wire current $i_{w}$ remains essentially constant, even as $R_{w}$ changes slightly. With the nanowire exposed to the flow, we adjust the bridge voltage $U_{0}$ until the desired overheat ratio $a=R_{w}=R_{w ; 20^{\circ} \mathrm{C}}$ is reached with typical values of $a=1.2-1.4$ corresponding to wire overheat temperatures of $100^{\circ} \mathrm{C}-200^{\circ} \mathrm{C}$. The resistance $R_{p}$ is chosen such that the bridge is balanced at working conditions. The bridge voltage is then proportional to small differences in $R_{w}$ that come about as the time varying cooling by the flow changes the wire temperature slightly. Amplified by a factor $K=100$ via an instrumentation amplifier, the bridge voltage $E_{b}$ is recorded as the output parameter of the CCA using an analogue-digital converter (ADC). A calibration and additional signal processing (e.g. filtering) as required finally yield the desired measurement of the fluctuating fluid velocity.

The nanowire was tested in the Max Planck VDTT in Göttingen described elsewhere ${ }^{24}$. The device was placed in the freestream behind an active turbulence generating grid as sketched in Figure $8 \mathrm{~b}$. The grid triggers turbulent motion in the fluid such that the fluid velocity $v(t)$ at the hotwire location fluctuates in time around its mean $V$. For the present set of measurements, the VDTT was operated at a pressure $p=2$ bar with $\mathrm{SF}_{6}$ at a temperature of $21^{\circ} \mathrm{C}$ as the working medium. The overheat ratio was set to $a=1.24$ and the wire current was $i_{w}=0.622 \mathrm{~mA}$.

\section{ACKNOWLEDGEMENTS}

This work is supported by the Max Planck-University of Twente Center for Complex Fluid Dynamics and by the Netherlands Organisation for Scientific Research (NWO) Gravitation programme funded by the Ministry of Education, Culture and Science of the government of the Netherlands. 
Free-standing Pt nanowires

\section{COMPETING INTERESTS}

The authors declare no conflict of interest

\section{SUPPLEMENTARY INFORMATION}

Supplementary information accompanies the manuscript on the Microsystems \& Nanoengineering website: http://www.nature.com/micronano.

\section{REFERENCES}

1. Pope, S. B. Turbulent Flows. (Cambridge University Press, 2000).

2. Grossmann, S. \& Lohse, D. Characteristic scales in Rayleigh-Benard turbulence. Phys. Lett. A 173, 58-62 (1993).

3. Emsellem, V., Kadanoff, L. P., Lohse, D., Tabeling, P. \& Wang, Z. J. Transitions and probes in turbulent helium. Phys. Rev. E - Stat. Physics, Plasmas, Fluids, Relat. Interdiscip. Top. 55, 2672-2681 (1997).

4. Hutchins, N., Nickels, T. B., Marusic, I. \& Chong, M. S. Hot-wire spatial resolution issues in wall-bounded turbulence. J. Fluid Mech. 635, 103-136 (2009).

5. Perry, A. E. Hot-wire anemometry. Oxford Oxford Univ. Press. (1982).

6. Bruun, H. H. Hot-wire anemometry. Oxford Oxford Univ. Press. (1995).

7. Tropea, C, Yarin, A. L. \& F. J. Springer handbook of experimental fluid mechanics. Berlin: Springer (2007).

8. Comte-Bellot, G. Hot-wire anemometry. Annu. Rev. 209-231 (1976).

9. Ligrani, P. M. \& Bradshaw, P. Spatial resolution and measurement of turbulence in the viscous sublayer using subminiature hot-wire probes. Exp. Fluids 5, 407-417 (1987).

10. Hultmark, M., Ashok, A. \& Smits, A. J. A new criterion for end-conduction effects in hot-wire anemometry. Meas. Sci. Technol. 22, 055401 (2011). 
Free-standing Pt nanowires

11. Willmarth, W. W. \& Sharma, L. K. Study of turbulent structure with hot wires smaller than the viscous length. J. Fluid Mech. 142, 121-149 (1984).

12. Löfdahl, L., Stemme, G. \& Johansson, B. Silicon based flow sensors used for mean velocity and turbulence measurements. Exp. Fluids 12, 270-276 (1992).

13. Jiang, F., Tai, Y.-C., Ho, C.-M. \& Li, W. J. A micromachined polysilicon hot-wire anemometer. in Solid state sensor and actuator workshop (1994).

14. Chen, J., Fan, Z., Zou, J., Engel, J. \& Liu, C. Two-dimensional micromachined flow sensor array for fluid mechanics studies. J. Aerosp. Eng. 16, 85-97 (2003).

15. Kunkel, G. J., Arnold, C. B. \& Smits, A. J. Development of NSTAP: Nanoscale thermal anemometry probe. J. Microelectromechanical Syst. 3, 1938-1945 (2006).

16. Bailey, S. C. C. et al. Turbulence measurements using a nanoscale thermal anemometry probe. J. Fluid Mech. 663, 160-179 (2010).

17. Vallikivi, M. \& Smits, A. J. Fabrication and characterization of a novel nanoscale thermal anemometry probe. J. Microelectromechanical Syst. 23, 899-907 (2014).

18. Fan, Y., Arwatz, G., Van Buren, T. W., Hoffman, D. E. \& Hultmark, M. Nanoscale sensing devices for turbulence measurements. Exp. Fluids 56, 1-13 (2015).

19. Fu, M. K., Fan, Y. \& Hultmark, M. Design and validation of a nanoscale cross - wire probe ( X - NSTAP ). Exp. Fluids 1-14 (2019). doi:10.1007/s00348-019-2743-0

20. Borisenkov, Y., Kholmyansky, M., Krylov, S., Liberzon, A. \& Tsinober, A. Multiarray Micromachined Probe for Turbulence Measurements Assembled of Suspended Hot-Film Sensors. J. Microelectromechanical Syst. 24, 1503-1509 (2015).

21. Diribarne, P., Thibault, P. \& Roche, P. E. Nano-shaped hot-wire for ultra-high resolution anemometry in cryogenic helium. Rev. Sci. Instrum. 90, (2019).

22. Puczylowski, J., Hölling, A., Peinke, J., Bhiladvala, R. \& Hölling, M. A new approach to highly resolved measurements of turbulent flow. Meas. Sci. Technol. 26, 1-12 (2015). 
Free-standing Pt nanowires

23. Salort, J., Monfardini, A. \& Roche, P. E. Cantilever anemometer based on a superconducting micro-resonator: Application to superfluid turbulence. Rev. Sci. Instrum. 83, (2012).

24. Bodenschatz, E., Bewley, G. P., Nobach, H., Sinhuber, M. \& Xu, H. Variable density turbulence tunnel facility. Rev. Sci. Instrum. 85, 093908 (2014).

25. Talluru, K. M., Kulandaivelu, V., Hutchins, N. \& Marusic, I. A calibration technique to correct sensor drift issues in hot-wire anemometry. Meas. Sci. Technol. 25, (2014).

26. Hutchins, N., Monty, J. P., Hultmark, M. \& Smits, A. J. A direct measure of the frequency response of hot-wire anemometers: temporal resolution issues in wallbounded turbulence. Exp. Fluids 56, (2015).

27. Le-The, H. et al. Shrinkage Control of Photoresist for Large-Area Fabrication of Sub-30 nm Periodic Nanocolumns. Adv. Mater. Technol. 2, (2017).

28. Le-The, H. et al. Large-scale fabrication of highly ordered sub-20 nm noble metal nanoparticles on silica substrates without metallic adhesion layers. Microsystems Nanoeng. 4, 4 (2018). 